\title{
Wiang Haeng Coal-Water Fuel Preparation and Gasification, Thailand - Task 39
}

\author{
Topical Report \\ July 1996
}

\author{
By \\ Chris M. Anderson \\ Mark A. Musich \\ Brian C. Young \\ John J. Richter \\ Ray A. DeWall \\ Robert O. Ness, Jr.
}

Work Performed Under Contract No.: DE-FC21-93MC30098

For

U.S. Department of Energy

Office of Fossil Energy

Morgantown Energy Technology Center

P.O. Box 880

Morgantown, West Virginia 26507-0880

RECEIVED

AUG 141997

OSTI

\section{By}

Energy and Environmental Research Center

University of North Dakota

15 North 23rd Street

P. O. Box 9018

Grand Forks, North Dakota 58202-9018

ISTRIBUTION OF THIS DOCUMENT IS UNLIMITED 


\section{Disclaimer}

This report was prepared as an account of work sponsored by an agency of the United States Government. Neither the United States Government nor any agency thereof, nor any of their employees, makes any warranty, express or implied, or assumes any legal liability or responsibility for the accuracy, completeness, or usefulness of any information, apparatus, product, or process disclosed, or represents that its use would not infringe privately owned rights. Reference herein to any specific commercial product, process, or service by trade name, trademark, manufacturer, or otherwise does not necessarily constitute or imply its endorsement, recommendation, or favoring by the United States Government or any agency thereof. The views and opinions of authors expressed herein do not necessarily state or reflect those of the United States Government or any agency thereof. 


\section{DISCLAIMER}

Portions of this document may be illegible electronic image products. Images are produced from the best available original document. 


\section{TABLE OF CONTENTS}

LIST OF FIGURES $\ldots \ldots \ldots \ldots \ldots \ldots \ldots \ldots \ldots \ldots \ldots \ldots \ldots \ldots \ldots \ldots \ldots$

LIST OF TABLES $\ldots \ldots \ldots \ldots \ldots \ldots \ldots \ldots \ldots \ldots \ldots \ldots \ldots \ldots \ldots \ldots \ldots$

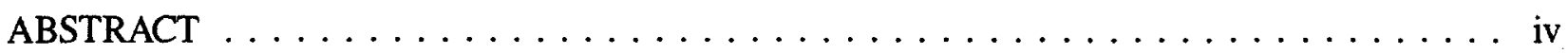

EXECUTIVE SUMMARY $\ldots \ldots \ldots \ldots \ldots \ldots \ldots \ldots \ldots \ldots \ldots \ldots \ldots$ iv

CHAPTER 1. INTRODUCTION $\ldots \ldots \ldots \ldots \ldots \ldots \ldots \ldots \ldots \ldots \ldots \ldots \ldots \ldots \ldots \ldots \ldots$

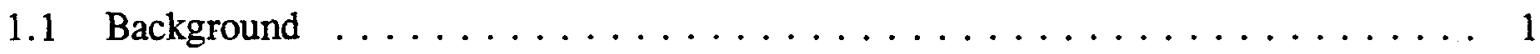

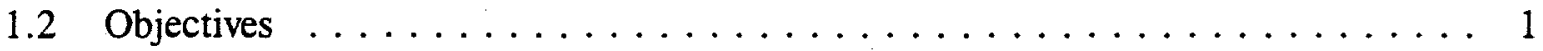

CHAPTER 2. EXPERIMENTAL PROCEDURES AND RESULTS $\ldots \ldots \ldots \ldots \ldots \ldots 2$

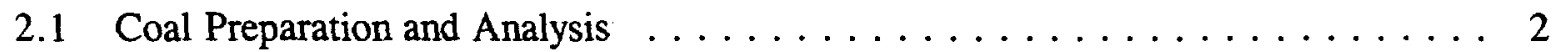

2.2 Bench-Scale HWD . . . . . . . . . . . . . . . . . . 4

2.3 Pilot-Scale HWD $\ldots \ldots \ldots \ldots \ldots \ldots \ldots \ldots \ldots \ldots \ldots \ldots \ldots$

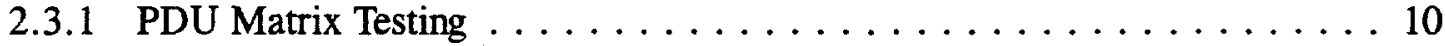

2.3.2 PDU Production Testing and CWF Preparation ........... 13

2.4 CWF Gasification and Utilization of By-Products $\ldots \ldots \ldots \ldots \ldots \ldots \ldots$

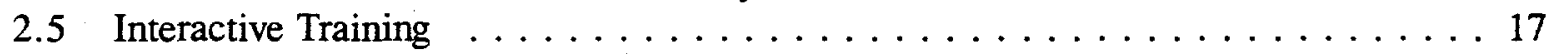

CHAPTER 3. STORAGE AND TRANSPORTATION OF CWF $\ldots \ldots \ldots \ldots \ldots \ldots 18$

3.1 CWF Pumpability Review . . . . . . . . . . . . . . . . 18

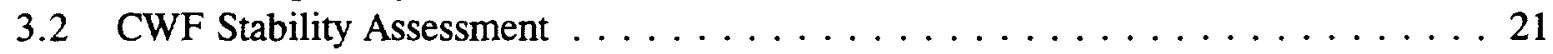

CHAPTER 4. CONCEPTUALIZED CWF DEVELOPMENT PLAN $\ldots \ldots \ldots \ldots \ldots 22$

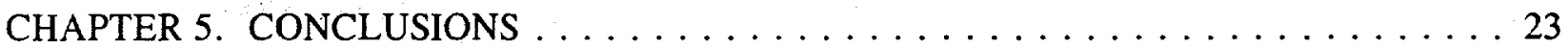

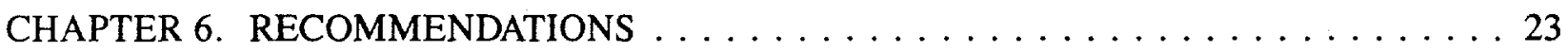

ANALYTICAL EQUIPMENT AND PROCEDURES $\ldots \ldots \ldots \ldots \ldots \ldots$ Appendix A AUTOCLAVE TEMPERATURE AND PRESSURE TRENDS $\ldots \ldots \ldots \ldots \ldots$ Appendix B RHEOLOGICAL PROFILES FOR COAL-WATER FUELS $\ldots \ldots \ldots \ldots \ldots$ Appendix C HOT-WATER-DRYING PILOT-SCALE INFORMATION $\ldots \ldots \ldots \ldots \ldots \ldots$ Appendix D HWD PROCESS GAS AND WATER ANALYSIS $\ldots \ldots \ldots \ldots \ldots \ldots$ Appendix E

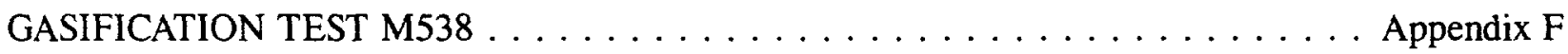

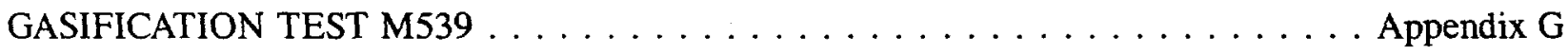




\section{LIST OF FIGURES}

1 Development plan for preparation and gasification of CWF $\ldots \ldots \ldots \ldots \ldots$

2 Schematic diagram of autoclave HWD system $\ldots \ldots \ldots \ldots \ldots \ldots$

3 Rheological analysis for raw and HWD coal samples $\ldots \ldots \ldots \ldots \ldots \ldots$

4 Rheological analysis for raw and HWD coal samples $\ldots \ldots \ldots \ldots \ldots \ldots$

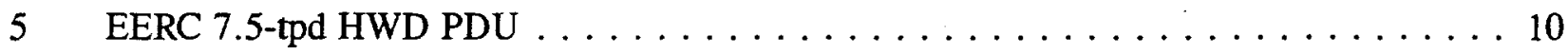

6 Rheological analysis for coal samples $\ldots \ldots \ldots \ldots \ldots \ldots \ldots \ldots \ldots \ldots \ldots \ldots$

$7 \quad$ Rheological analysis for coal samples $\ldots \ldots \ldots \ldots \ldots \ldots \ldots \ldots \ldots \ldots \ldots$

8 Continuous fluid-bed reactor utilized for gasification testing $\ldots \ldots \ldots \ldots \ldots \ldots$

$9 \quad$ DMR personnel training activities for February $1996 \ldots \ldots \ldots \ldots \ldots \ldots$

10 Pump requirements for transporting $3.5 \mathrm{MM}$ tons/yr of Wiang Haeng coal CWF $\ldots \ldots 20$

11 Energy requirements for transporting $3.5 \mathrm{MM}$ tons/yr of Wiang Haeng coal CWF. . . . 21

12 Rheological results for raw coal slurry and enhanced CWF $\ldots \ldots \ldots \ldots \ldots \ldots 22$

\section{LIST OF TABLES}

1. Chemical Analysis Results for Wiang Haeng Coal $\ldots \ldots \ldots \ldots \ldots \ldots$

2 Analysis Results for Wiang Haeng Coal Washability Testing $\ldots \ldots \ldots \ldots \ldots$

3 Particle-Size Distribution Analysis for Raw and HWD Samples $\ldots \ldots \ldots \ldots \ldots$

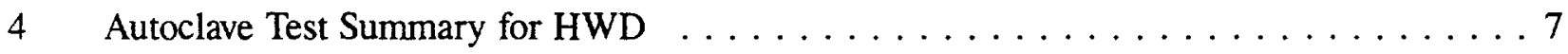

5 Equilibrium Moisture, Proximate and Ultimate Analysis, Heating Value, and Ash Analysis for Raw Coal and HWD Coal Samples $\ldots \ldots \ldots \ldots \ldots \ldots$

6 Short Proximate Analysis for Raw and PDU HWD Coal Samples $\ldots \ldots \ldots \ldots \ldots \ldots$

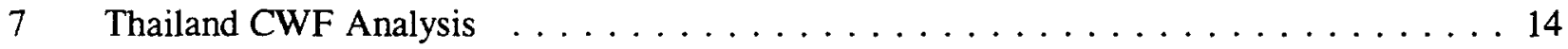

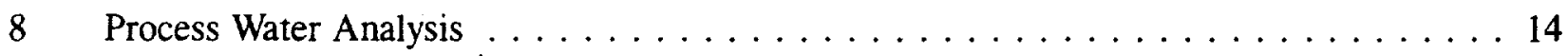




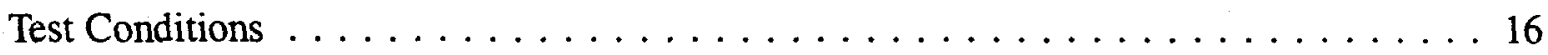

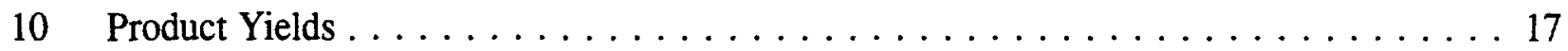

11 Product and Cyclone Char Proximate Analysis $\ldots \ldots \ldots \ldots \ldots \ldots \ldots$

12 Rod Penetrometer Test Results on Raw and HWD Samples $\ldots \ldots \ldots \ldots \ldots \ldots 22$ 


\title{
WIANG HAENG COAL-WATER FUEL PREPARATION AND GASIFICATION, THAILAND
}

\begin{abstract}
In response to an inquiry by the Department of Mineral Resources (DMR) in Thailand, the Energy \& Environmental Research Center (EERC) prepared a four-task program to assess the responsiveness of Wiang Haeng coal to the temperature and pressure conditions of hot-water drying (HWD). The results indicate that HWD made several improvements in the coal, notably increases in heating value and carbon content and reductions in equilibrium moisture and oxygen content. The equilibrium moisture content decreased from $37.4 \mathrm{wt} \%$ for the raw coal to about $20 \mathrm{wt} \%$ for the HWD coals. The energy density for a pumpable coal-water fuel indicates an increase from 4450 to $6650 \mathrm{Btu} / \mathrm{lb}$ by hydrothermal treatment. Raw and HWD coal were then gasified at various mild gasification conditions of $700^{\circ} \mathrm{C}$ and $30 \mathrm{psig}$. The tests indicated that the coal is probably similar to other low-rank coals, will produce high levels of hydrogen, and be fairly reactive.
\end{abstract}

\section{EXECUTIVE SUMMARY}

In Thailand, coal is the major source for both power and nonpower usages. Over the past 10 years, the Department of Mineral Resources (DMR) in Thailand has been evaluating the ability of the country's coal reserves to meet its increasing utility and industrial energy needs. DMR discovered over 750 million tons of measured coal and subbituminous reserves. The majority of the reserves are the subject of additional exploration and development plans, which include applying clean coal technologies (i.e., coal preparation and beneficiation techniques) to reduce the impact of coal use on the environment.

DMR and other governmental groups in Thailand are leading the charge to this new coal utilization policy. In September of 1993, officials organized a Clean Coal Technology seminar in Chiangmai and a public workshop in Bangkok. As a result of these meetings, technology needs were identified to enhance coal utilization. One need identified is to produce a coal-water fuel (CWF) from coal for gasification systems. CWFs are the most promising of all alternative coalbased fuels. Converting coal into an easily transported liquid fuel could make it an ideal candidate to replace costly imported oil. The economic merits of CWFs are attractive because, on a heatingunit basis, coal is cheaper than oil. Its quasi-liquid form maintains that differential, since the higher cost of dry bulk coal handling and storage is avoided. The CWF technology opens up new markets to coal producers, while it offers price stability and security of supply for the fuel end users.

The Energy \& Environmental Research Center (EERC), which has for years been investigating the conversion of coal to energy-dense liquid fuels, was identified as a leading candidate to perform the development program. In response to the inquiry, the EERC prepared a four-task program to assess the responsiveness of the Wiang Haeng coal to the temperature and pressure conditions of hot-water drying (HWD). The treated material was to be slurried in water and gasified at various conditions. The remaining activity focused on Thai personnel training at the EERC in the area of clean coal technologies. The project terms and conditions were accepted in October 1995. Approximately $600 \mathrm{~kg}$ of lignite was received in October. Coal was sized and 
analyzed for testing. A series of six bench-scale hot-water-drying (HWD) tests were completed in October and November. Results indicate an improvement of energy density (slurry basis) from 4450 to $6650 \mathrm{Btu} / \mathrm{lb}$ using the EERC-developed HWD technology.

Pilot-scale HWD tests were performed at $300^{\circ}$ and $325^{\circ} \mathrm{C}$ and 7 - and 15 -minute residence times. Results indicated an improvement of energy density (slurry fuel basis) from 4360 to $5830 \mathrm{Btu} / \mathrm{lb}$ at $300^{\circ} \mathrm{C}$ and $6050 \mathrm{Btu} / \mathrm{lb}$ at $325^{\circ} \mathrm{C}$ and a 7 -minute residence time. The effect of increasing residence time to 15 minutes was to further increase the heating value to 5980 and $6130 \mathrm{Btu} / \mathrm{lb}$ at $300^{\circ}$ and $325^{\circ} \mathrm{C}$, respectively.

A pilot-scale $\mathrm{HWD}$ production run was performed at $325^{\circ} \mathrm{C}$ with a 7 -minute residence time. All HWD coal produced at $325^{\circ} \mathrm{C}$ was formulated into approximately $245 \mathrm{~kg}$ (540 lb) of CWF for subsequent gasification testing. The CWF had a solids concentration of $50.8 \mathrm{wt} \%$, an estimated heating value of $6200 \mathrm{Btu} / \mathrm{lb}$, and a viscosity of approximately $510 \mathrm{cP}$.

Mild gasification tests were conducted using raw and HWD Wiang Haeng coal at $700^{\circ} \mathrm{C}$ at 30 psig. Two different coal sizes were used in the investigation and two steam:carbon ratios. Gas production was approximately $30 \%$ based on maf feed. There was a net loss of water from the process, which indicates that the water-gas shift reaction was occurring. Hydrogen production was very high, accounting for $57 \%-59 \%$ of the total gas production. No clear conclusion can be made on the impact of HWD the coal prior to gasification because of the differences in gasification conditions.

Based on the success of CWF experiments, the next stages of the development may include testing new coals or testing CWF in a combustion system. Also, additional data are required to evaluate the storage and transportation properties of the CWF. Continued success may lead to extended pilot-scale testing and eventually to commercial demonstration in Thailand.

Specific tasks the EERC and DMR may consider for future gasification program development include the need to define products for specific gasifier types: slurry fuel, solid fuel product (briquettes, fine char), synfuel, or electrical production. Future product testing may include an evaluation of the amount of gas, char, and tar produced at various temperature conditions. Bench-scale tests would then be conducted to optimize tar production versus volatile content of the char. In order to consider synfuel and electrical production opportunities, laboratory tests may include thermogravimetric analysis matrix testing to investigate steam reactivity. Also, a more thorough ash characterization would be needed to assess slagging characteristics.

Personnel from Thailand's DMR completed interactive training by observing bench- and pilot-scale demonstrations in coal cleaning, briquetting, HWD, CWF preparation and evaluation, and CWF gasification. 


\section{WIANG HAENG COAL-WATER FUEL PREPARATION \\ AND GASIFICATION, THAILAND}

\section{CHAPTER 1. INTRODUCTION}

\subsection{Background}

Low-rank coal (LRC) is a carbonaceous material that has not undergone a sufficient geological metamorphosis to convert it into a high-volatile bituminous coal. The incomplete coalification process results in a high moisture content because of the porous nature of the coal. To beneficiate LRC requires a significant reduction in moisture; i.e., the coal must be dried.

Unfortunately, proven technologies used to remove surface moisture and improve the heating value of bituminous coals are not effective on LRC. The evaporative processes employed in conventional methods for drying bituminous coal involve rapid drying, and under such conditions, LRC disintegrates. This creates a dust nuisance and an increased risk of spontaneous combustion. A further disadvantage is that moisture is quickly reabsorbed when the LRC is exposed to humid air or slurried in water.

During the 1970s, researchers began to use elevated temperature and pressure as a means of producing coal-water fuel from lignites. In simple terms, the process, known as hydrothermal treatment or hot-water drying (HWD), induces coalification in a condensed time scale of minutes rather than geological eras (millions of years), thus effecting a permanent reduction in inherent moisture. In other words, the lignite is changed from hydrophilic to hydrophobic, thus making it similar to some bituminous coals.

As a result of earlier investigations, the Energy \& Environmental Research Center (EERC) has developed an economical method of upgrading LRC based on the hydrothermal treatment process. The technical feasibility of this new, nonevaporative technique has been established in the EERC's 7.5-tpd pilot plant, and commercial demonstration of the technology is currently in the planning stage.

\subsection{Objectives}

The EERC, at Grand Forks, North Dakota, with support from the U.S. Department of Energy (DOE), entered into a jointly sponsored research project with the Thailand Department of Mineral Resources (DMR) to investigate the application of an EERC-developed nonevaporative hydrothermal drying process, HWD, to coal from the chosen Thailand deposit. The evaluation included determining the gasification characteristics and efficiencies of the potential fuels. Figure 1 presents a time line for the main objectives, which are listed below:

- Investigate the hydrothermal treatment conditions for the selected Wiang Haeng coal at the bench and pilot scale.

- Evaluate the gasification performance of the hydrothermally treated Wiang Haeng coal slurry. 


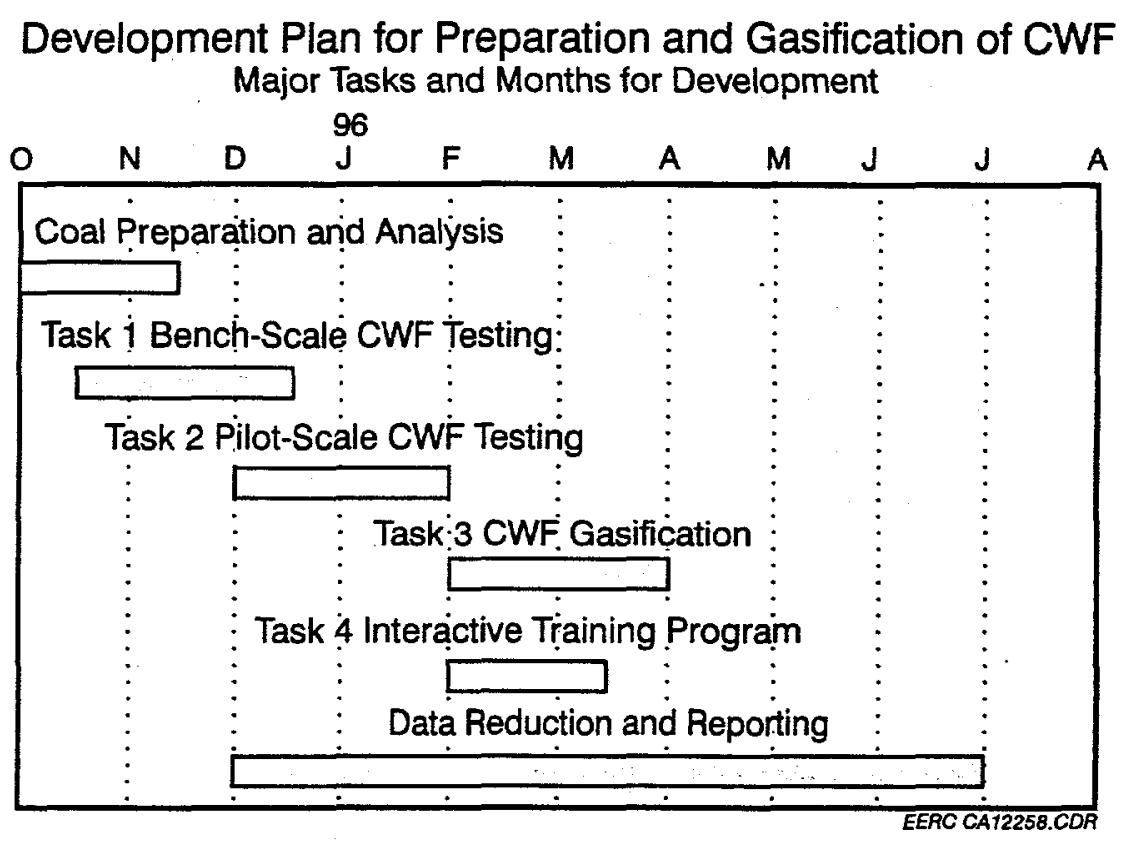

Figure 1. Development plan for preparation and gasification of CWF.

- Provide an interactive technology transfer training program on coal technology for coal applications.

\section{CHAPTER 2. EXPERIMENTAL PROCEDURES AND RESULTS}

\subsection{Coal Preparation and Analysis}

Approximately $600 \mathrm{~kg}$ of Wiang Haeng coal was received. The coal was somewhat slacked in appearance, indicating that the sample was not fresh prior to shipment or that some drying occurred during transit. The Wiang Haeng coal, received as lumps up to $0.25 \mathrm{~m}$ (estimated), was crushed with a roll crusher, producing nominal $4 \mathrm{~cm} \times 0 \mathrm{~cm}$ coal. A $45-\mathrm{kg}$ composite sample was generated, and approximately $543 \mathrm{~kg}$ of presized coal was reserved for pilot-scale testing. Test samples for analyses and bench-scale evaluations were prepared from the composite sample. The analysis fraction of the presized as-received (AR) Wiang Haeng coal was submitted for proximate, ultimate, heating value, equilibrium moisture, sulfur forms, $x$-ray fluorescence analysis (XRFA), and ash fusion determinations. Analytical equipment and methods are presented in Table A-1 (Appendix A), and resulting data are presented in Table 1 below for the AR Wiang Haeng coal.

The Wiang Haeng coal has a low ash content (6.5 wt\% moisture-free [mf]), an acidic-type ash (over $92 \mathrm{wt} \%$ oxides of silicon, aluminum, and iron), high sulfur and nitrogen contents (2.0 and $1.8 \mathrm{wt} \% \mathrm{mf}$, respectively), and an equilibrium moisture content of $37.3 \mathrm{wt} \%$ (relative to an 
AR value of $31.0 \mathrm{wt} \%)$. Sulfur forms analysis indicated that only $22 \mathrm{wt} \%$ of total sulfur is present as pyrite.

\section{TABLE 1}

Chemical Analysis Results for Wiang Haeng Coal

\begin{tabular}{|c|c|c|c|c|}
\hline Analysis & As-Received & Moisture ${ }^{1}$ & Moisture-Free & $\begin{array}{l}\text { Moisture- and } \\
\text { Ash-Free }\end{array}$ \\
\hline \multicolumn{5}{|l|}{ Proximate, wt $\%$} \\
\hline Moisture & 31.00 & 37.33 & -- & -- \\
\hline Volatile Matter & 31.64 & 28.73 & 45.85 & 49.04 \\
\hline Fixed Carbon & 32.87 & 29.86 & 47.64 & 50.96 \\
\hline Ash & 4.49 & 4.08 & 6.51 & -- \\
\hline \multicolumn{5}{|l|}{ Ultimate, wt\% } \\
\hline Hydrogen & 6.70 & 7.11 & 4.72 & 5.05 \\
\hline Carbon & 46.61 & 42.33 & 67.55 & 72.25 \\
\hline Nitrogen & 1.15 & 1.05 & 1.67 & 1.78 \\
\hline Sulfur & 1.29 & 1.18 & 1.88 & 2.01 \\
\hline Oxygen & 39.75 & 44.25 & 17.67 & 18.91 \\
\hline Ash & 4.49 & 4.08 & 6.51 & -- \\
\hline \multicolumn{5}{|c|}{ Higher Heating Value } \\
\hline $\mathrm{MJ} / \mathrm{kg}$ & 18.7 & 17.0 & 27.1 & 28.9 \\
\hline Btu/lb & 8020 & 7290 & 11,630 & 12,430 \\
\hline \multicolumn{5}{|l|}{ Sulfur Forms, wt\% } \\
\hline Organic & 0.84 & 0.78 & 1.25 & 1.34 \\
\hline Pyritic & 0.26 & 0.24 & 0.39 & 0.42 \\
\hline Sulfatic & 0.09 & 0.08 & 0.13 & 0.14 \\
\hline \multicolumn{5}{|c|}{ Sulfur Dioxide Emission } \\
\hline $\mathrm{g} / \mathrm{MJ}$ & 1.39 & 1.39 & 1.39 & 1.39 \\
\hline $\mathrm{lb} / \mathrm{MMBtu}$ & 3.23 & 3.23 & 3.23 & 3.23 \\
\hline
\end{tabular}

${ }^{1}$ Equilibrium moisture basis.

The 3.8- $\times 0$-cm coal was stage-crushed to $6.35 \times 0 \mathrm{~mm}$ using a roller mill and then screened at $20 \mathrm{mesh}$. The $6.35-\times 0$-mm mesh sample along with the -20 -mesh fines were submitted for short proximate (moisture, ash, sulfur, heating value) analysis.

The $6.35-\times 0$-mm Wiang Haeng coal was subjected to washability analysis to determine the release of ash (as minerals) and sulfur (as pyrite) under wet-density-based conditions. Float-sink testing using true (homogeneous) heavy liquids was performed at specific gravities of $1.3,1.4$, and 1.6. Static float-sink analysis was performed on the $-6.35-\mathrm{mm} \times 20$-mesh coal by placing a 100 to 150 -gram sample in a float-sink flask containing $1600 \mathrm{~mL}$ of 1.3 specific gravity Certigrav solution. The test was considered complete when the coal separated into distinct float (clean coal) and sink (minerals and pyrite) refuse fractions. The sink fraction was subjected to separation at higher specific gravity (1.4), with this procedure repeated until a total of three separations was performed. The products of float-sink testing included three float fractions and one sink fraction, 
which were ethanol-washed, air-dried, weighed, and then submitted for short proximate analysis. The washability results for the $-6.35 \mathrm{~mm} \times 20$-mesh Wiang Haeng coal are presented in Table 2 . Calculated values include coal and Btu recovery and ash and sulfur reduction as a function of specific gravity.

\section{TABLE 2}

Analysis Results for Wiang Haeng Coal Washability Testing (moisture-free)

\begin{tabular}{lccccc}
\hline & $\begin{array}{c}\text { Coal } \\
\text { Recovery, } \\
\%\end{array}$ & $\begin{array}{c}\text { Btu } \\
\text { Recovery, } \\
\%\end{array}$ & $\begin{array}{c}\text { Ash } \\
\text { Content, } \\
\text { wt } \%\end{array}$ & $\begin{array}{c}\text { Sulfur } \\
\text { Content, } \\
\text { wt\% }\end{array}$ & $\begin{array}{c}\text { Heating } \\
\text { Value, } \\
\text { Btu/lb }\end{array}$ \\
\hline Raw Coal & & & 6.51 & 1.88 & 11,630 \\
-20 mesh & & & 7.97 & 2.12 & 11,310 \\
$-6.35 \mathrm{~mm} \times 20$ mesh & & & 5.74 & 1.89 & 11,840 \\
Direct & & & & & \\
+1.3 Float & 75.99 & 79.76 & 2.18 & 1.38 & 12,410 \\
1.3-1.4 Float & 9.91 & 9.32 & 7.52 & 2.40 & 11,119 \\
1.4-1.6 Float & 11.12 & 9.38 & 15.94 & 2.90 & 9974 \\
-1.6 Sink & 2.99 & 1.55 & 43.94 & 4.30 & 6125 \\
Cumulative & & & & & 12,410 \\
+1.3 Float & 75.99 & 79.76 & 2.18 & 1.38 & 12,261 \\
+1.4 Float & 85.90 & 89.08 & 2.80 & 1.50 & 11,999 \\
+1.6 Float & 97.01 & 98.45 & 4.30 & 1.66 & 11,823 \\
\hline Total & 100 & 100 & 5.49 & 1.74 & \\
\hline
\end{tabular}

\section{$2.2^{\circ}$ Bench-Scale HWD}

The Wiang Haeng coal was roller mill-crushed to produce feed for autoclave HWD. Two particle-size distributions (PSD) were produced from the $-6.35-\times 0-\mathrm{mm}$ coal. The bench-scale HWD tests with Wiang Haeng coal were performed in the EERC's 7.6-liter batch autoclave shown in Figure 2. The bolted closure autoclave is externally heated and equipped with automatic temperature controllers and a variable-speed magnetically driven stirrer. The autoclave is instrumented to continuously measure and trend pressure plus slurry and vapor temperatures.

Approximately 3000 grams of a $50 \mathrm{wt} \% \mathrm{coal} / 50 \mathrm{wt} \%$ deionized water feed slurry was used in each HWD test. After feed slurry was charged to the autoclave, residual air was evacuated and the external heaters and stirrer turned on. Heatup to the desired temperature ranged from approximately 2 to $2 \frac{1}{2}$ hours, after which the slurry and vapor temperatures were allowed to stabilize. After a 15-minute hold time at temperature, the heaters were shut off, and the autoclave and contents were allowed to cool overnight. 


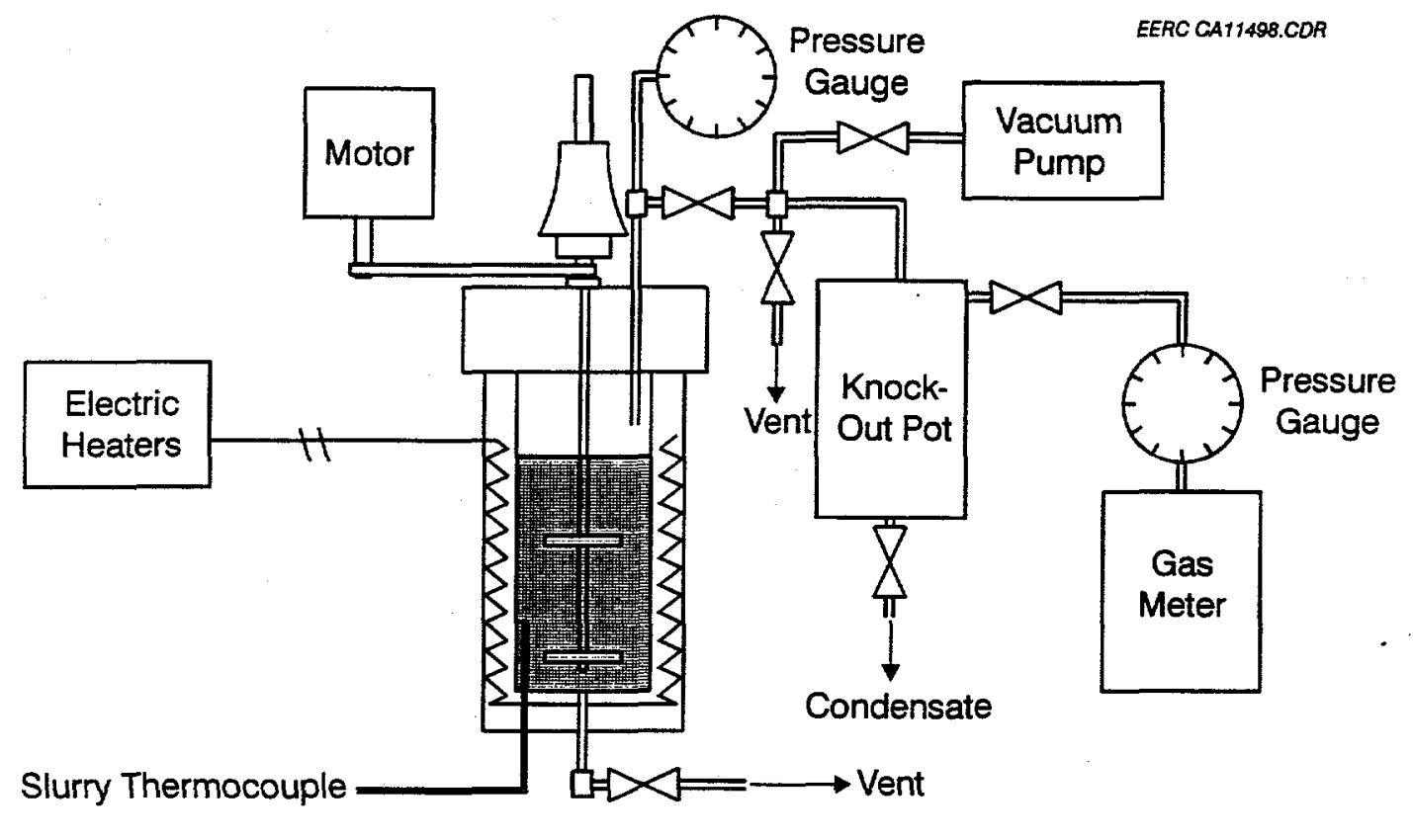

Figure 2. Schematic diagram of autoclave HWD system.

Fractions recovered from the autoclave after cooldown included process gas, product slurry, and condensate. The total volume of process gas (noncondensable decomposition products) was measured with a diaphragm meter. Process gas was sampled for off-line analysis. The product slurry was recovered, weighed, and then dewatered via Büchner filtration. Liquid samples (filtrate and condensate) were refrigerated prior to analysis to inhibit biological activity.

A total of six tests were performed; variables evaluated included PSD and treatment temperature. 'The HWD-treated solids were then reslurried in water and evaluated for size distribution and rheological performance. The PSD is important, because if the particles are too large, there will be more void spaces and lower packing efficiency, resulting in a decreased fuel solids concentration. The rheological behavior for the coal-liquid mixture, specifically the apparent viscosity, is determined as a function of shear rate and coal-water fuel (CWF) solids concentrations. Since any change in solids loading of a CWF has a direct effect on the resulting flow behavior, several rheograms throughout the possible solids-loading range were gathered. The characterization protocol for products of hydrothermal treatment is presented in Table A-2 (Appendix A), and analytical equipment and methods are presented in Table A-1 (Appendix A).

The first two autoclave tests were performed using a -60 -mesh sample and a -200 -mesh sample. Figure 3 illustrates that $-250 \mu \mathrm{m}(60$-mesh) coal produced a fuel 2 to $3 \mathrm{wt} \%$ higher than the $-75-\mu \mathrm{m}$ (200 mesh) coal in solids loading, which was attributed to a broader PSD resulting in more efficient particle packing. HWD tests were then performed with $-250-\mu \mathrm{m}$ coal at $275^{\circ}$, $300^{\circ}$, and $325^{\circ} \mathrm{C}$. Duplicate tests were also performed at $275^{\circ}$ and $325^{\circ} \mathrm{C}$. Table 3 summarizes 
the PSD analysis on raw coal and treated samples. Relatively no change was realized from HWD treatment. Appendix B summarizes the temperatures and pressure conditions during autoclave is.

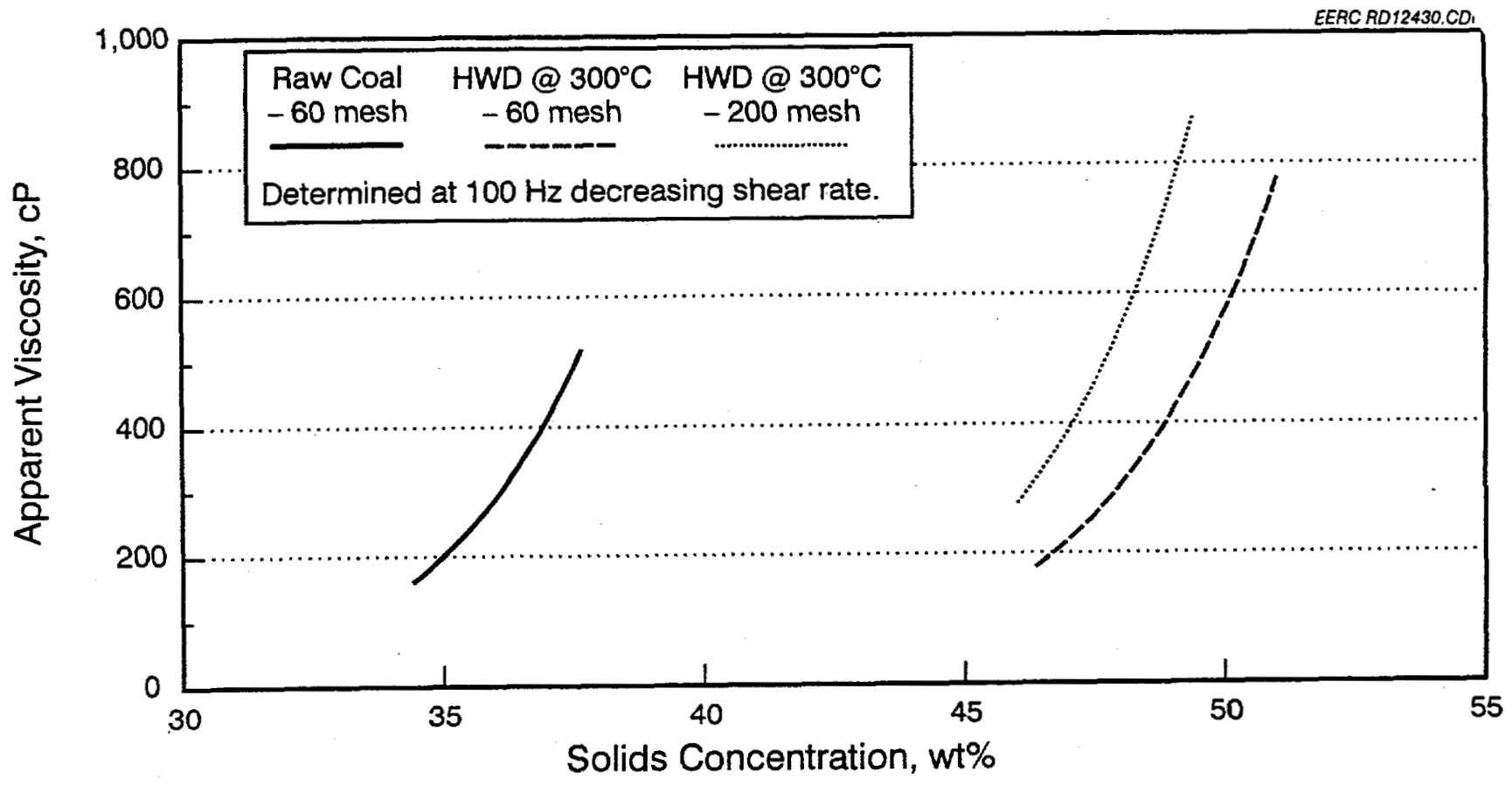

Figure 3. Rheological analysis for raw and HWD coal samples (particle-size effect),

TABLE 3

Particle-Size Distribution Analysis for Raw and HWD Samples

\begin{tabular}{|c|c|c|c|c|}
\hline \multirow[b]{2}{*}{ Particle Size,$\mu \mathrm{m}$} & \multicolumn{4}{|c|}{ Cumulative wt \% under Size } \\
\hline & $\begin{array}{l}\text { Raw } \\
\text { Coal }\end{array}$ & $\begin{array}{l}\text { HWD, } \\
275^{\circ} \mathrm{C}\end{array}$ & $\begin{array}{l}\mathrm{HWD}, \\
300^{\circ} \mathrm{C}\end{array}$ & $\begin{array}{l}\mathrm{HWD}, \\
325^{\circ} \mathrm{C}\end{array}$ \\
\hline $500-$ & 100.0 & 100.0 & 100.0 & 100.0 \\
\hline 300 & 100.0 & 100.0 & 100.0 & 100.0 \\
\hline 150 & 98.2 & 98.0 & 97.9 & 98.5 \\
\hline 100 & 89.0 & 88.0 & 87.0 & 85.8 \\
\hline 70 & 75.1 & 74.1 & 75.2 & 75.0 \\
\hline 50 & 64.4 & 62.5 & 60.8 & 63.5 \\
\hline 25 & 45.4 & 41.6 & 39.0 & 44.0 \\
\hline 15 & 32.7 & 27.9 & 26.5 & 31.7 \\
\hline 10 & 23.1 & 17.6 & 17.3 & 21.5 \\
\hline 5 & 9.9 & 6.9 & 7.0 & 9.9 \\
\hline 2 & 0.5 & 0.2 & 0.2 & 0.4 \\
\hline Estimated $\mathrm{MMD}^{\mathrm{I}}$ & 30 & 33 & 35 & 31 \\
\hline
\end{tabular}

'Mass mean diameter or average particle size. 
Table 4 contains a summary of the results from the autoclave tests performed on samples. The table contains the solids recovery and material converted to the gas and water phases. Solids and energy recoveries for each of the four samples were above $90 \%$. Duplicate test results at $275^{\circ}$ and $300^{\circ} \mathrm{C}$ illustrate the reproducibility of the procedures and their effects. Tests were performed on the HWD process water to evaluate the amount of carbonaceous material, the recyclability of the stream, and best treatment methods. Water analysis indicated that the total organic carbon (TOC), chemical oxygen demand (COD), and biological oxygen demand (BOD) concentrations increased as the temperature increased. Suspended solids decreased from 4500 to $1300 \mathrm{mg} / \mathrm{L}$ at $325^{\circ} \mathrm{C}$, while dissolved solids increased from 230 to $1300 \mathrm{mg} / \mathrm{L}$ at $325^{\circ} \mathrm{C}$. Gas analysis was performed using a Hewlett-Packard 5880a gas chromatograph to identify the gases produced during HWD. The results indicate that about $95 \mathrm{wt} \%$ of the gas evolved as $\mathrm{CO}_{2}$. Details of the instrumentation and techniques performed on the water and gas can be located in Appendix A. Coal losses mentioned in Table 4 represent all solids that were in the process water and offgases produced during the process. It is estimated that $90 \%$ of coal losses were attributed to process gas. As temperature increased, solids recovery decreased; however, energy recovery remained high. This is likely because $\mathrm{CO}_{2}$ represents a bulk of the loss from the dry solid that contributes no heating value.

\section{TABLE 4}

Autoclave Test Summary for HWD

\begin{tabular}{|c|c|c|c|c|c|c|}
\hline Con & $\begin{array}{l}\text { HWD, } \\
275^{\circ} \mathrm{C}\end{array}$ & $\begin{array}{l}\text { HWD, } \\
275^{\circ} \mathrm{C}\end{array}$ & $\begin{array}{l}\text { HWD, } \\
300^{\circ} \mathrm{C}\end{array}$ & $\begin{array}{l}\text { HWD, } \\
300^{\circ} \mathrm{C}\end{array}$ & $\begin{array}{l}\text { HWD, } \\
325^{\circ} \mathrm{C}\end{array}$ & $\begin{array}{l}\text { HWD, } \\
325^{\circ} \mathrm{C}\end{array}$ \\
\hline Solids Recovery, \% & 94.2 & 96.5 & 93.1 & 93.2 & 91.7 & 93.1 \\
\hline Coal Loss, $\%$ & 5.8 & 3.5 & 6.9 & 6.8 & 8.3 & 6.9 \\
\hline Energy Recovery, \% & 98.7 & 98.7 & 98.5 & 97.9 & 99.9 & 100.3 \\
\hline
\end{tabular}

Table 5 summarizes the proximate, ultimate, and heating value analyses for the raw and the HWD samples. The results indicate that HWD made several improvements in the coal, notably increases in heating value and carbon content and reductions in equilibrium moisture and oxygen content. The equilibrium moisture content decreased from $37.4 \mathrm{wt} \%$ for the raw coal to about $20 \mathrm{wt} \%$ for the HWD coals. The moisture-free heating value of the raw coal, 11,630 Btu/lb, increased to $12,080 \mathrm{Btu} / \mathrm{lb}$ after $\mathrm{HWD}$ at $275^{\circ} \mathrm{C}$, and $\mathrm{HWD}$ at $325^{\circ} \mathrm{C}$ increased the heating value to nearly $12,500 \mathrm{Btu} / \mathrm{lb}$. The increase in heating value upon HWD was caused primarily by the reduction in oxygen content from decarboxylation and mild pyrolysis. Reported heating values on an equilibrium moisture basis reflect the drying that occurred to the coal, increasing from 7280 to $9820 \mathrm{Btu} / \mathrm{lb}$ at $300^{\circ} \mathrm{C}$.

Ash analysis for the HWD Thailand samples is also included in Table 5. The results indicate no substantial differences in the ash for the raw and three HWD temperature samples, except for elimination of calcium and reduction in sulfur and phosphorus concentrations. No change occurred to the ash fusion characteristics of the coal from hydrothermal treatment. 


\section{TABLE 5}

Equilibrium Moisture, Proximate and Ultimate Analysis, Heating Value, and Ash Analysis for Raw Coal and HWD Coal Samples

\begin{tabular}{|c|c|c|c|c|}
\hline Analysis & Raw Coal & $\begin{array}{l}\mathrm{HWD} \\
275^{\circ} \mathrm{C}\end{array}$ & $\begin{array}{l}\mathrm{HWD}, \\
300^{\circ} \mathrm{C}\end{array}$ & $\begin{array}{l}\mathrm{HWD}, \\
325^{\circ} \mathrm{C}\end{array}$ \\
\hline Equilibrium Moisture, wt\% & 37.4 & 23.4 & 19.4 & 21.9 \\
\hline \multicolumn{5}{|l|}{ Proximate, $\mathrm{mf}$, wt $\%$} \\
\hline Volatile Matter & 45.9 & 43.3 & 41.7 & 39.8 \\
\hline Fixed Carbon & 47.6 & 51.2 & 52.0 & 53.7 \\
\hline Ash & 6.5 & 5.5 & 6.3 & 6.6 \\
\hline \multicolumn{5}{|l|}{ Ultimate, $\mathrm{mf}, \mathrm{wt} \%$} \\
\hline Carbon & 67.6 & 72.3 & 72.6 & 73.9 \\
\hline Hydrogen & 4.7 & 4.8 & 4.5 & 4.7 \\
\hline Nitrogen & 1.7 & 1.7 & 1.8 & 1.8 \\
\hline Sulfur & 1.7 & 1.7 & 1.7 & 1.8 \\
\hline Oxygen & 17.7 & 14.1 & 13.0 & 11.3 \\
\hline \multicolumn{5}{|l|}{ Higher Heating Value, Btu/lb } \\
\hline Moisture-Free & 11,630 & 12,080 & 12,310 & 12,480 \\
\hline @ Equil. Moisture & 7280 & 9250 & 9820 & 9750 \\
\hline \multicolumn{5}{|l|}{$\begin{array}{l}\text { Ash Component, mf, wt } \% \\
\text { (as oxides) }\end{array}$} \\
\hline Silicon & 35.9 & 40.2 & 38.9 & 39.1 \\
\hline Aluminum & 25.1 & 26.5 & 25.8 & 25.5 \\
\hline Iron & 31.2 & 28.2 & 29.7 & 30.0 \\
\hline Titanium & 0.5 & 0.9 & 0.9 & 0.9 \\
\hline Phosphorus & 0.8 & 0.2 & 0.1 & 0.2 \\
\hline Calcium & 2.1 & 0.0 & 0.0 & 0.0 \\
\hline Magnesium & 1.5 & 1.6 & 2.0 & 1.8 \\
\hline Sodium & 0.2 & 0.2 & 0.2 & 0.2 \\
\hline Potassium & 1.4 & 1.5 & 1.5 & 1.5 \\
\hline Sulfur & 1.2 & 0.8 & 0.9 & 0.8 \\
\hline \multicolumn{5}{|l|}{ Ash Fusion, ${ }^{\circ} \mathrm{C}$} \\
\hline Initial Temperature & 1437 & NA & NA & 1448 \\
\hline Softening Temperature & 1459 & NA & NA & 1469 \\
\hline Hemi Temperature & 1468 & NA & NA & 1506 \\
\hline Fluid Temperature & +1538 & NA & NA & +1538 \\
\hline
\end{tabular}

The rheological profiles in Figure 4 show the effect of HWD temperature on CWF solids concentration and viscosity. The results indicate that HWD definitely improves the solids concentration of the fuel. The differences that are observed at $275^{\circ}$ and $325^{\circ} \mathrm{C}$ are attributed to decarboxylation, a greater amount occurring at the higher temperature. There is a definite improvement from $\mathrm{HWD}$ at $300^{\circ} \mathrm{C}$ compared to $275^{\circ} \mathrm{C}$; however, only slight improvement between $300^{\circ}$ and $325^{\circ} \mathrm{C}$. More information on the rheology of the CWFs is located in Appendix C. 


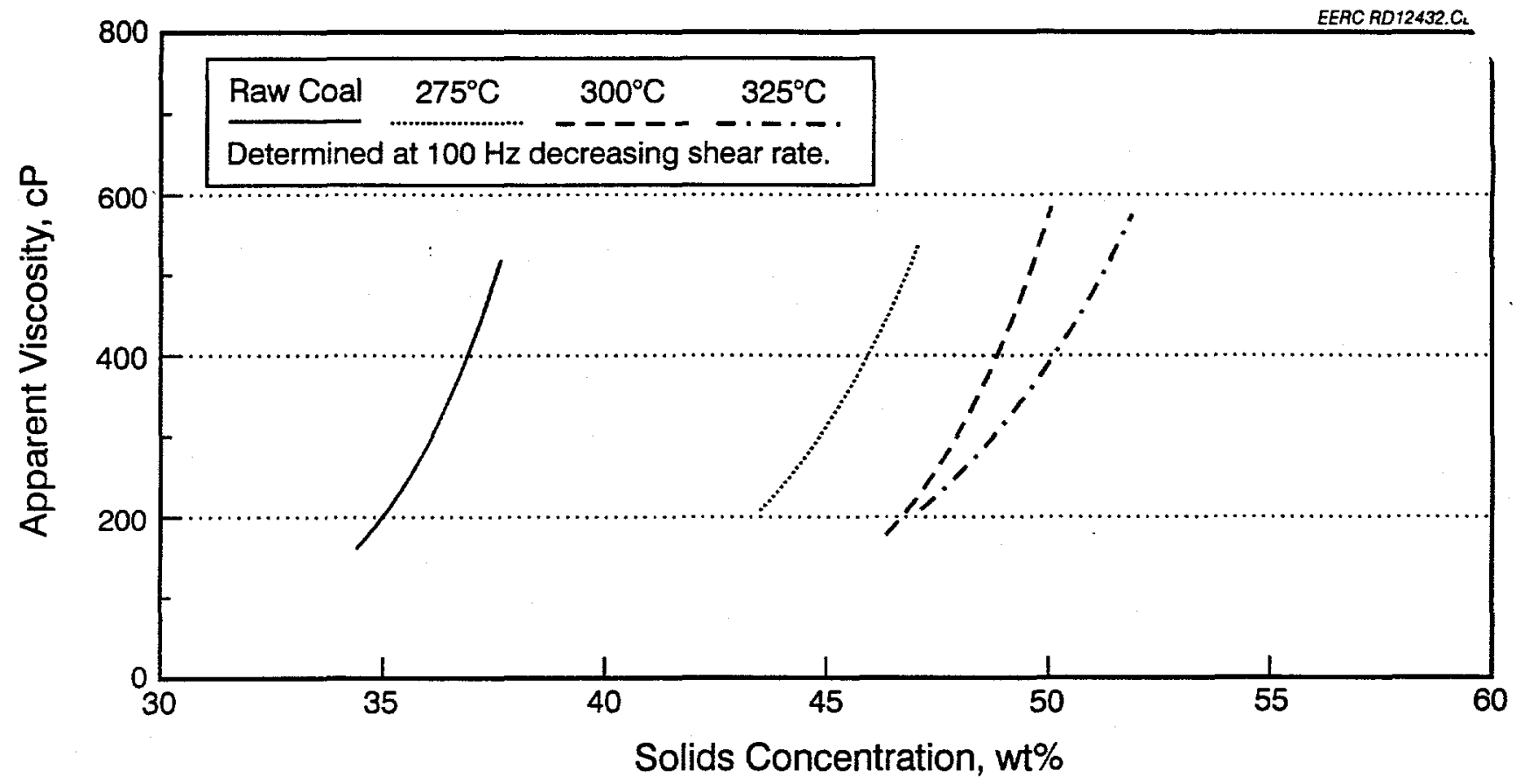

Figure 4. Rheological analysis for raw and HWD coal samples (temperature effect).

\subsection{Pilot-Scale HWD}

Wiang Haeng coal was processed using the 7.5-tpd HWD process development unit (PDU) shown in Figure 5. Briefly, the PDU system consists of the following major unit operations: slurry preparation, pumping, preheat, reaction, pressure letdown, product recovery, and HWD coal dewatering.

The coal to be processed is first pulverized with a hammer mill and then slurried to the desired coal-to-water ratio. A high-pressure pump, capable of pumping highly viscous feed slurries up to $2500 \mathrm{mPa}$-s is used to deliver the slurry to the preheat section at the desired system operating pressure. Slurry flow rate is controlled using a variable-speed motor.

A series of four heat exchangers is used to preheat the slurry to the desired processing temperature. A double-pipe steam heat exchanger first heats the slurry up to $80^{\circ} \mathrm{C}$, whereafter the slurry is heated in a series of three condensing Dowtherm vapor-liquid heat exchangers. The nominal ratings of the electric immersion heaters are 22,22 , and $30 \mathrm{~kW}$, respectively.

The slurry, after exiting the fourth preheater at the desired processing temperature, is then directed to a series of two downflow reactors. The process piping is configured to allow using a single reactor to attain a residence time of 7 minutes or both reactors to attain a residence time of 


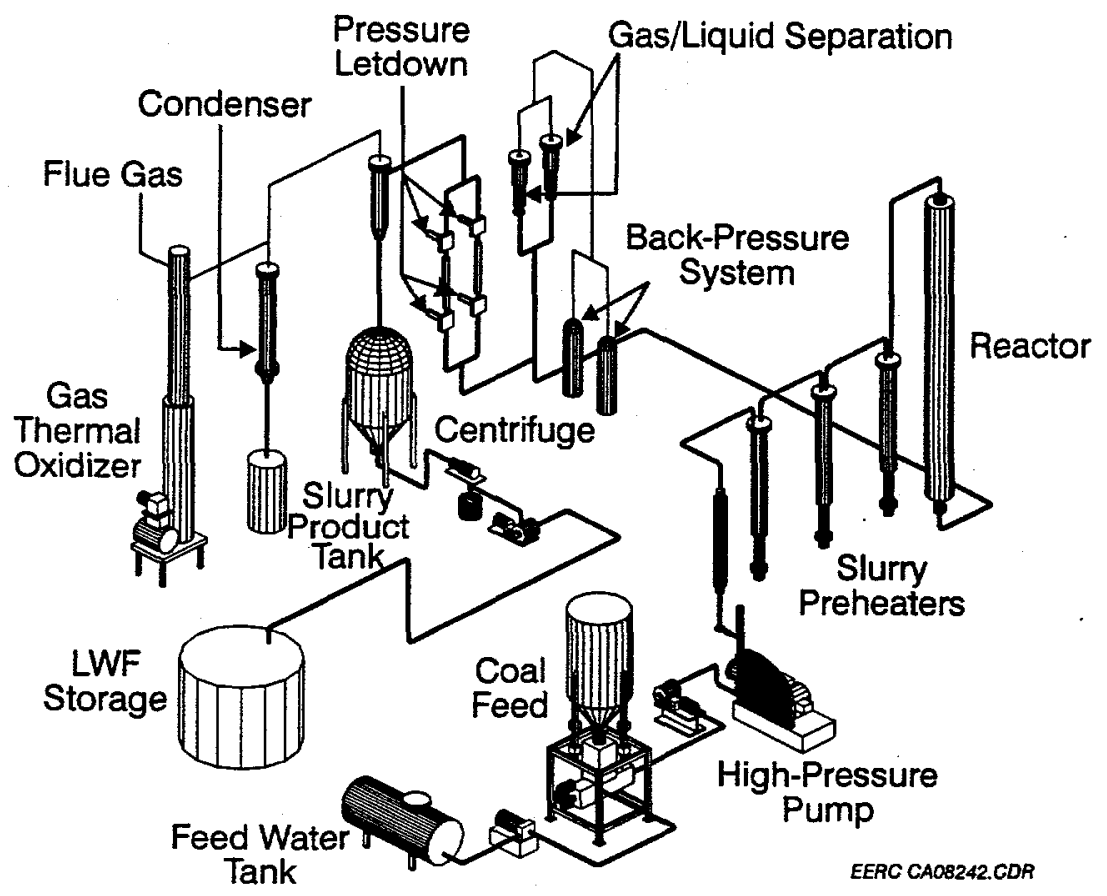

Figure 5. EERC 7.5-tpd HWD PDU.

15 minutes. Each reactor is equipped with four $2-\mathrm{kW}$ externally mounted heaters to achieve isothermal temperature control.

After processing at the desired temperature and residence time, the coal slurry is throttled through pressure-reducing valves with a resultant flashing of steam and process gas. The gas-water vapor stream is cyclonically separated from the concentrated HWD coal slurry. The gas-water vapor stream is drawn through a multipass water-cooled condenser whereafter the noncondensable process gas phase is sent to a natural gas-fired incinerator fired at $800^{\circ} \mathrm{C}$. Condensate is collected for possible recycle. The product slurry is dewatered using a recessed filter press, producing damp filter cake and filtrate.

\subsubsection{PDU Matrix Testing}

Pilot-scale matrix test parameters were based upon the results from bench-scale evaluations, discussed under Task 1 in the October to December progress report. Tests were completed at two residence times ( 7 and 15 minutes) and two temperatures $\left(300^{\circ}\right.$ and $325^{\circ} \mathrm{C}$ ). The Wiang Haeng coal, pulverized to $250-\mu \mathrm{m}$ (60-mesh) top size and an average size of $30 \mu \mathrm{m}$, was processed at a $45 \% / 55 \%$ coal-to-water ratio. (For more information on conditions see Appendix D). 
The weights of feed slurry, product slurry, condensate, filter cake, and filtrate were collected at each test condition to facilitate calculation of overall material and solids recoveries.

A representative filter cake sample was obtained at each test condition for subsequent short proximate (moisture, ash, sulfur, and heating value) analysis. Gas chromatographic analyses were performed on select samples of process gas.

Rheological evaluations were performed by admixing the damp cake from HWD with water to produce several different fuel concentrations. The rheological behavior of the slurry fuel, including variation of apparent viscosity as a function of shear rate, was determined for three or four solids concentrations using a Haake RV 100 viscometer.

Table 6 summarizes the ash, sulfur, and heating value analysis results for the four PDU HWD samples; the raw coal is included for comparison. The results indicate that HWD made several improvements to the Wiang Haeng coal, including significant reductions in ash and sulfur contents and a modest increase in heating value.

\section{TABLE 6}

Short Proximate Analysis for Raw and PDU HWD Coal Samples

\begin{tabular}{lccccc}
\hline & & $300^{\circ} \mathrm{C}$ & $325^{\circ} \mathrm{C}$ & $300^{\circ} \mathrm{C}$ & $325^{\circ} \mathrm{C}$ \\
Analysis & Raw Coal & $7 \mathrm{~min}$ & $7 \mathrm{~min}$ & $15 \mathrm{~min}$ & $15 \mathrm{~min}$ \\
\hline $\begin{array}{l}\text { Ash, mf } \\
\text { wt\% }\end{array}$ & 6.5 & 5.4 & 5.6 & 5.3 & 5.5 \\
$\begin{array}{l}\text { Sulfur, } \mathrm{mf} \\
\text { wt\% }\end{array}$ & 1.88 & 1.47 & 1.51 & 1.59 & 1.61 \\
$\begin{array}{l}\mathrm{HHV}{ }^{2} \mathrm{mf} \\
\text { Bu/lb }\end{array}$ & 11,630 & 12,230 & 12,300 & 12,300 & 12,380 \\
\hline & & & & & \\
'Moisture-free. & & & &
\end{tabular}

The increases in heating value appeared to be consistent with the effect of increasing the processing temperature and/or residence time. That is, the lowest improvement in heating value was attained at the lowest residence time and processing temperature, and the greatest improvement in heating value was attained at the highest residence time and processing temperature. Processing at $325^{\circ} \mathrm{C}$ and a 7 -minute residence time or $300^{\circ} \mathrm{C}$ and a 15 -minute residence time produced an equivalent heating value product.

HWD produced a significant reduction in sulfur content, ranging from $22 \mathrm{wt} \%$ at $300^{\circ} \mathrm{C}$ and 7 minutes to $14 \mathrm{wt} \%$ at $325^{\circ} \mathrm{C}$ and a 15 -minute residence time. The lower sulfur reduction at the most severe processing conditions would be consistent with greater coal mass loss to pyrolysis and decarboxylation relative to less severe processing conditions. 
The solids recoveries for the four tests ranged from 88 to $93 \mathrm{wt} \%$. These values were lower than bench-scale HWD recovery values but consistent with previous PDU operation.

The rheological profiles in Figures 6 and 7 show the effect of PDU HWD temperature (300 and $325^{\circ} \mathrm{C}$, respectively) on CWF solids concentration and viscosity. The rheological profiles for bench-scale HWD at the respective temperatures are included for comparison. Similarly to benchscale HWD, PDU processing did significantly improve the solids content (and energy density) of the Wiang Haeng coal.

Comparison of energy density (slurry basis), for fuels with a viscosity of $500 \mathrm{cP}$, showed an increase from 4360 to $5830 \mathrm{Btu} / \mathrm{lb}$ at $300^{\circ} \mathrm{C}$ and $6050 \mathrm{Btu} / \mathrm{lb}$ at $325^{\circ} \mathrm{C}$ and a 7 -minute residence time. The effect of increasing residence time to 15 minutes was to further increase the heating value, resulting in slurry fuels with energy densities of 5980 and $6130 \mathrm{Btu} / \mathrm{lb}$ at $300^{\circ}$ and $325^{\circ} \mathrm{C}$, respectively.

Relative to bench-scale HWD, pilot-scale HWD produced fuels 1 to $2 \mathrm{wt} \%$ lower in solids loading. This can be explained by the differences in residence time achieved during batch- and continuous-scale processing. During pilot-scale testing, operating temperature is reached after approximately 2 minutes, while bench-scale heatup takes approximately 2 hours. Bench-scale fuel performance results are usually better than pilot-scale results because of the extended time in which the coal is exposed to temperatures above $200^{\circ} \mathrm{C}$.

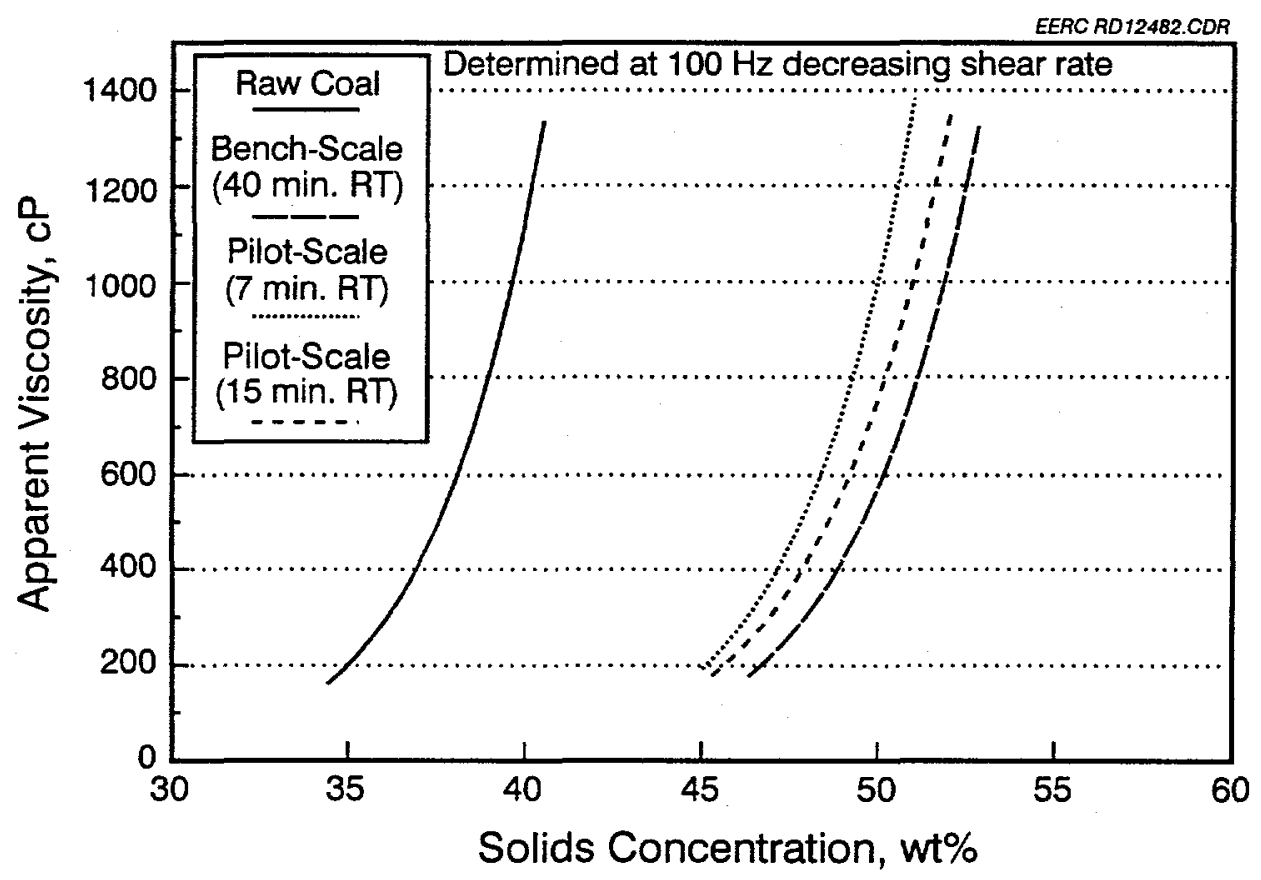

Figure 6. Rheological analysis for coal samples (bench vs. pilot scale, $300^{\circ} \mathrm{C}$ tests). 


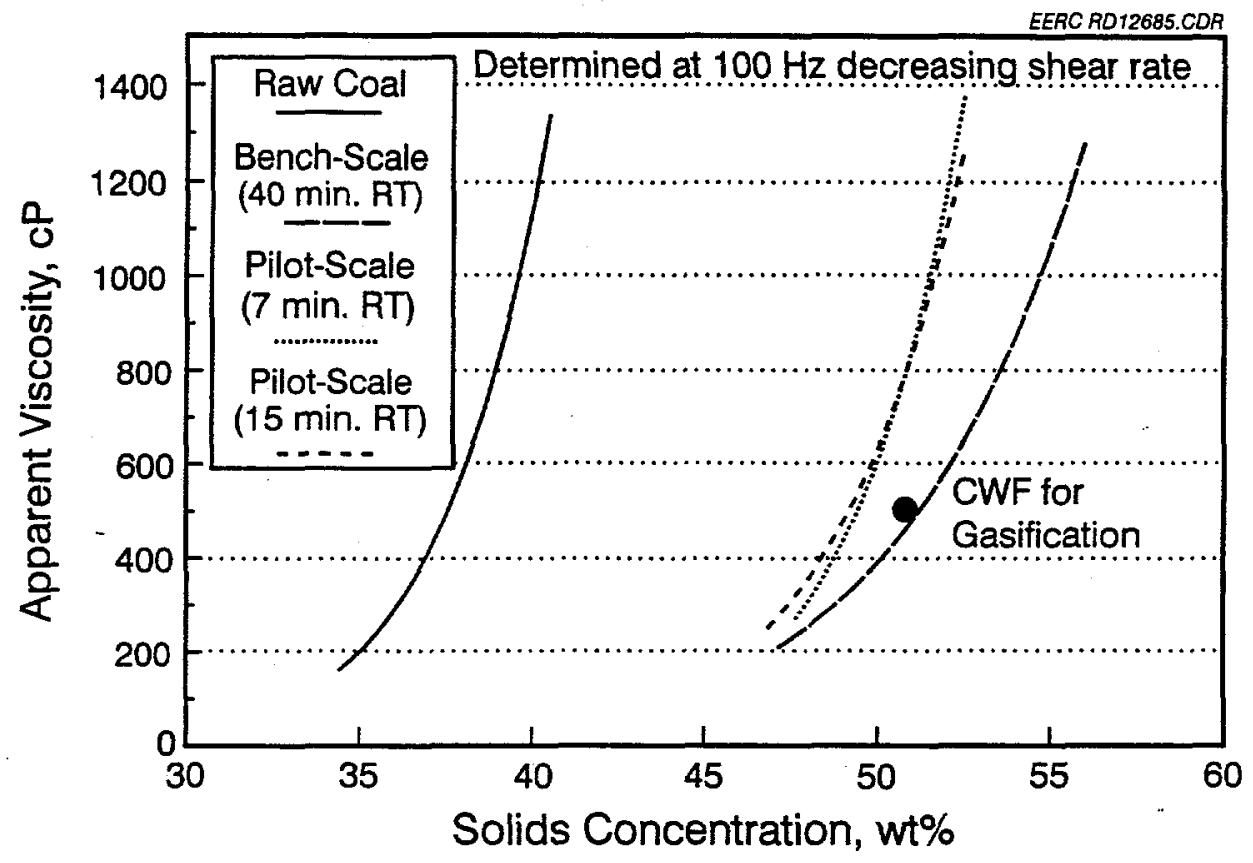

Figure 7. Rheological analysis for coal samples (bench vs. pilot scale, $325^{\circ} \mathrm{C}$ tests).

Process gas samples obtained during processing at $325^{\circ} \mathrm{C}$ (at both 7 - and 15 -minute residence times) were nearly equivalent in composition. Carbon dioxide was the primary component at $>93 \mathrm{vol} \%$, with smaller concentrations of hydrogen $(\sim 0.3 \mathrm{vol} \%)$, methane $(\sim 1 \mathrm{vol} \%)$, and carbon monoxide ( $\sim 1$ to $2 \mathrm{vol} \%)$. The balance $(\sim 3 \mathrm{vol} \%)$ consisted of mostly hydrogen sulfide. The quantity of process gas was not measured.

\subsubsection{PDU Production Testing and CWF Preparation}

A production PDU HWD test was performed at a 7-minute residence time and $325^{\circ} \mathrm{C}$, processing conditions determined to be optimum from pilot-scale matrix testing. The purpose of the run was to supplement the filter cake produced at $325^{\circ} \mathrm{C}$ (and 7- and 15 -minute residence times) in the PDU matrix tests. The solids recovery in the production test $(87 \mathrm{wt} \%)$ fell within the range of recovery values obtained in the PDU matrix tests. (For information on conditions see Appendix D).

HWD coal produced in the pilot-scale production run and $325^{\circ} \mathrm{C}$ matrix tests was formulated into CWF for subsequent gasification testing. Approximately $245 \mathrm{~kg}(540 \mathrm{lb})$ of CWF was prepared with a target viscosity of nominally $500 \mathrm{cP}$. The CWF had a solids concentration of $50.8 \mathrm{wt} \%$ and an estimated heating value of $6200 \mathrm{Btu} / \mathrm{lb}$. Fuels prepared in the PDU production run and matrix tests are compared in Figure 7 . The proximate, ultimate, and heating value analyses are presented on a moisture-free and slurry basis in Table 7 for the raw and $325^{\circ} \mathrm{C}$ HWD coals. Process water analyses are presented in Table 8 for the PDU HWD test at $325^{\circ} \mathrm{C}$. For more information on process water and process gas from HWD testing, consult Appendix E. 
TABLE 7

Thailand CWF Analysis

\begin{tabular}{lcccc}
\hline & \multicolumn{2}{c}{$325^{\circ} \mathrm{C}$ HWD } & \multicolumn{2}{c}{ Raw } \\
\cline { 2 - 5 } Analysis & $\begin{array}{c}\text { Moisture- } \\
\text { Free }\end{array}$ & CWF & Free & CWF \\
\hline Ultimate, wt\% & & & & \\
Moisture & 0.0 & 49.24 & 0.0 & 62.50 \\
Volatile Matter & 42.08 & 21.36 & 45.85 & 17.19 \\
Fixed Carbon & 51.99 & 26.39 & 47.64 & 17.87 \\
Ash & 5.93 & 3.01 & 6.51 & 2.44 \\
Proximate, wt\% & & & & \\
Hydrogen & 5.06 & 8.04 & 4.72 & 8.71 \\
Carbon & 74.56 & 37.85 & 67.55 & 25.33 \\
Nitrogen & 1.62 & 0.82 & 1.67 & 0.63 \\
Sulfur & 1.48 & 0.75 & 1.88 & 0.71 \\
Oxygen & 11.35 & 49.53 & 17.67 & 62.18 \\
Ash & 5.93 & 3.01 & 6.51 & 2.44 \\
Heating Value, Btu/lb & 12,210 & 6200 & 11,630 & 4360 \\
\hline
\end{tabular}

TABLE 8

Process Water Analysis, mg/L

\begin{tabular}{lrr}
\hline Analysis & Filtrate & Condensate \\
\hline COD & 1090 & 2650 \\
Total Suspended Solids & 370 & 220 \\
Total Dissolved Solids & 3860 & $<10$ \\
Total Organic Carbon & 475 & 894 \\
Total Carbon & 484 & 1060 \\
\hline
\end{tabular}




\subsection{CWF Gasification and Utilization of By-Products}

A diagram of the EERC 4-lb/hr pressurized fluid-bed gasifier utilized for gasification testing is presented in Figure 8. The properties of the CWF prepared for gasification testing were discussed in the previous section.

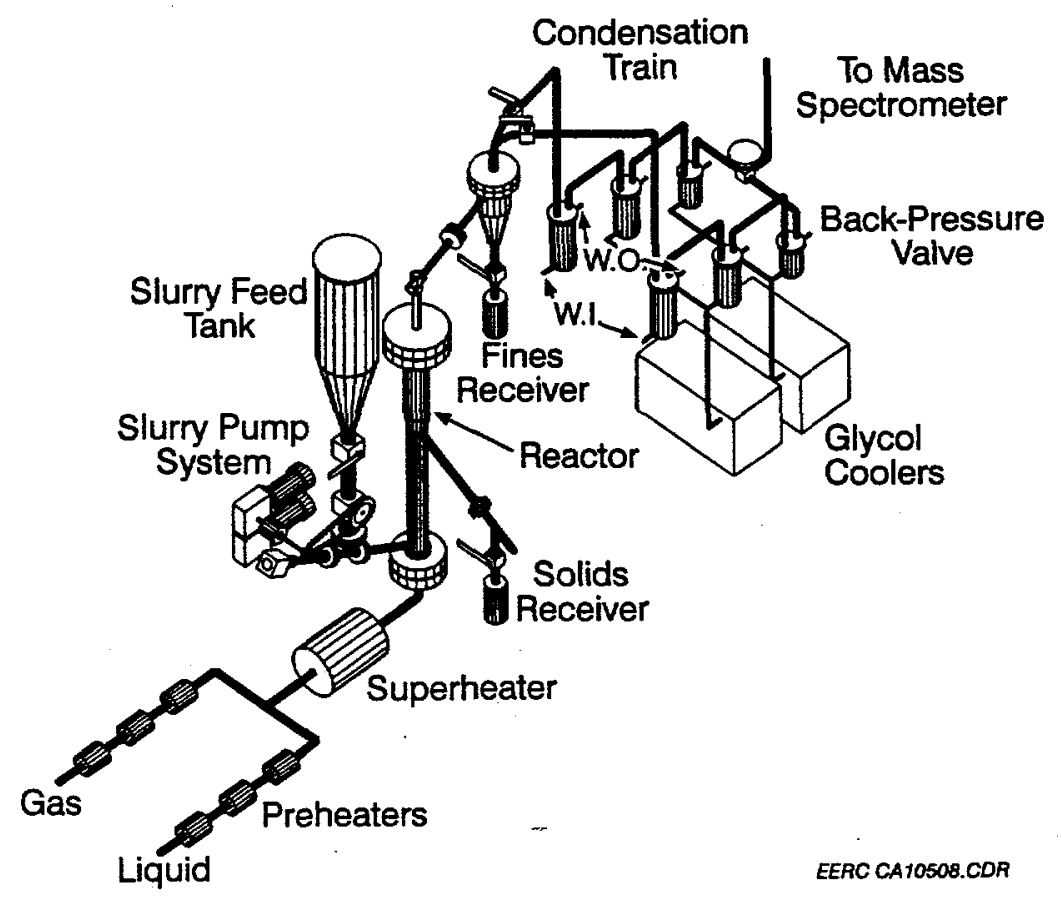

Figure 8. Continuous fluid-bed reactor utilized for gasification testing.

Difficulties were encountered in feeding the slurry into the gasifier, as solids frequently plugged the last inch of the nozzle through which CWF was injected into the gasifier. Several modifications were attempted to improve and prolong pumping, including trying different pumps, varying injection nozzle diameter, and varying injection techniques. Pluggage still occurred in as quickly as 5 minutes, with 4 hours of pumping achieved at the best conditions. This was still far short of the 12-hour run required to complete a single test point.

Consequently, the test program was modified to evaluate gasification of dry feed. Dry feed for gasification was obtained by air-drying the filter cake obtained during HWD at $325^{\circ} \mathrm{C}$. The CWF initially prepared for gasification testing was not utilized for preparation of the dry feed. Tests were also conducted using $1 / 4-\times 20$-mesh HWD sample. Raw Wiang Haeng coal will also be gasified to provide a comparison of the benefits of HWD.

Three tests were conducted using Wiang Haeng coal under mild gasification conditions. Two different coal sizes were used in the investigation and two steam:carbon ratios. All three tests were conducted at $700^{\circ} \mathrm{C}$ at $30 \mathrm{psig}$. The coal was fed to the system as a dry solid and not as a slurry because the type of gasifier used was a fluid bed, not an entrained reactor. One test (M537) used a 
particle size similar to the CWF reported earlier in this report, and the second (M538) and third (M539) tests were conducted on coal that was $-1 / 4 \times+20$ mesh. M538 was the raw coal, while M539 test was conducted on HWD sample.

M538 and M539 had complete material balances. M537 data were inconsistent, and yields could not be determined from the data due to feeding problems caused by the small particle size. Table 9 shows the test conditions from the two tests and Table 10 shows the product yields. Appendices $\mathrm{F}$ and $\mathrm{G}$ contain the full material balances and product data for Tests M538 and M539.

The temperature $\left(700^{\circ} \mathrm{C}\right)$ selected for operation was in the mild gasification range. Products from these conditions will include a low-moisture, volatile char, minor quantities of organic liquids, and low quantities of combustible gases. As can be seen from Table 11, char production in both tests was quite high (85.9-88.4). The increased solids residence time can be seen in the decrease in char volatile content from $15 \%$ to $10 \%$. Cyclone fines volatile content is relatively unchanged because the fine particles pass through the reactor quickly.

TABLE 9

Test Conditions

\begin{tabular}{lcc}
\hline & M538 & M539 \\
\cline { 2 - 3 } Temperature, ${ }^{\circ} \mathrm{C}$ & 699.7 & 694.2 \\
Pressure, psig & 29.7 & 29.8 \\
Steam:Feed Ratio & -1.86 & 2.53 \\
Fluid Velocity, ft/s & 0.72 & 0.72 \\
Solids Res. Time, $\min$ & 66 & 89 \\
\hline
\end{tabular}

Gas production was approximately $30 \%$ based on maf feed. There was a net loss of water from the process, which indicates that the water-gas shift reaction was occurring. Hydrogen production was very high, accounting for $57 \%-59 \%$ of the total gas production. No clear conclusion can be made on the impact of HWD the coal prior to gasification because of the differences in gasification conditions.

The significance of the test results cannot be fully evaluated without determining the final goal of the process: electrical production, char production, etc. In mild gasification, a high quantity of char was produced in a volatile range that is appropriate for the conditions stated. Organic tars were not analyzed for, so an estimate of binding for briquettes cannot be done. The conditions tested did not evaluate the potential for gasification for electrical production; however, the tests did indicate that the coal is probably similar to other low-rank coals and will produce high levels of hydrogen and be fairly reactive. A specific match for a particular gasifier (entrained, fluid bed, agglomerating, etc.) cannot be made without defining further process goals or products. 
TABLE 10

Product Yields

\begin{tabular}{lcc}
\hline & M538 & M539 \\
\cline { 2 - 3 } maf $^{1}$ Char Out & 85.9 & 88.4 \\
$\mathrm{H}_{2} \mathrm{O}$ & -17.7 & -23 \\
Ash & 0.9 & 3.6 \\
Gas & 30.9 & 31 \\
Total & 100.0 & 100
\end{tabular}

Gas Production

\begin{tabular}{lrl}
$\mathrm{H}_{2}$ & 0.2692 & 0.3297 \\
$\mathrm{CO}_{2}$ & 0.1159 & 0.0861 \\
$\mathrm{C}_{3} \mathrm{H}_{6}$ & 0.0041 & 0.0030 \\
$\mathrm{H}_{2} \mathrm{~S}$ & 0.0028 & 0.0059 \\
$\mathrm{C}_{2} \mathrm{H}_{4}$ & 0.0069 & 0.0059 \\
$\mathrm{C}_{2} \mathrm{H}_{6}$ & 0.0028 & 0.0030 \\
$\mathrm{~N}_{2}$ & -0.0014 & 0.0059 \\
$\mathrm{CH}_{4}$ & 0.0566 & 0.0861 \\
$\mathrm{CO}$ & 0.0179 & 0.0386 \\
Total, std. $\mathrm{m}^{3} / \mathrm{kg}$ & 0.4748 & 0.5642 \\
\hline
\end{tabular}

${ }^{1}$ Moisture and ash-free.

TABLE 11

Product and Cyclone Char Proximate Analysis

\begin{tabular}{lccrrrr}
\hline & & & \multicolumn{2}{c}{ Product } & \multicolumn{2}{c}{ Cyclone } \\
\cline { 4 - 7 } & AR Coal & mf Coal & M538 & M539 & M538 & M539 \\
\hline Moisture & 31.00 & - & 0.60 & 0.40 & 2.40 & 4.60 \\
Volatiles & 31.64 & 45.85 & 15.11 & 10.11 & 18.31 & 17.61 \\
Fixed Carbon & 32.87 & 47.64 & 76.89 & 79.86 & 70.27 & 65.31 \\
Ash & 4.49 & 6.51 & 7.40 & 9.36 & 9.02 & 12.49 \\
\hline
\end{tabular}

\subsection{Interactive Training}

The interactive training program, designed to provide first-hand experience with bench- and pilot-scale coal upgrading processes, was completed during the period from February 4 to 23. A calendar showing the training activities of DMR personnel is presented in Figure 9.

Mr. Somchai from the Thailand DMR observed bench-scale demonstrations in float-sink washability testing, autoclave HWD, CWF preparation and rheological evaluation, pressurized fluid-bed gasification and pilot-scale demonstrations in dense-media physical cleaning, PDU HWD, 
and roll-press briquetting. Further, Mr. Somchai, Mr. Kriangkrai, and Mr. Navee completed comprehensive tours of the EERC's laboratory and combustion facilities.

Although not part of the experimental test program, procedures and results were presented for the pilot-scale physical cleaning and briquetting demonstrations. Approximately $150 \mathrm{lb}$ of Wiang Haeng coal was sized to $1 / 4$-in. top size using a roller mill and then screened at $850 \mu \mathrm{m}$ (20 mesh). The sized coal was subjected to dense-media physical cleaning using a cone-type separator with a nominal capacity of $150 \mathrm{~kg} / \mathrm{hr}(330 \mathrm{lb} / \mathrm{hr})$. Pulverized magnetite, sized at $70 \mathrm{wt} \%$ $<45 \mu \mathrm{m}$ (325 mesh), was used to produce a specific gravity of 1.25 to 1.30 . The process was not optimized for the Wiang Haeng coal.

The recoveries of float, sink, and $-850-\mu \mathrm{m}(-20-\mathrm{mesh})$ coal were 62,30 , and $8 \mathrm{wt} \%$, respectively. The ash contents of the float and sink were 6.2 and $25.3 \mathrm{wt} \% \mathrm{mf}$, respectively, which compares to $6.5 \mathrm{wt} \% \mathrm{mf}$ for the as-received Wiang Haeng coal. The results indicate a concentration of ash-bearing minerals in the sink, although the float product did not produce a commensurately lower ash value.

Roll press briquetting demonstrations were performed using the float and sink products from physical cleaning as well as the HWD coal produced during the interactive training demonstration PDU test. Two briquetting tests were performed with the float product, and one test each was performed with the sink and HWD coal fractions. Nominally $8 \mathrm{wt} \%$ (dry basis) of a pregelatinized commercial potato starch was used as binder for each test.

The feed (coal and binder) was blended using a batch cement mixer and then densified using a double-roll press briquetter operated with a separation force of 15 tons. Approximately $50 \mathrm{lb}$ $(25 \mathrm{~kg}$ ) of material was processed in each test. The briquettes were air-dried and then stored; no analysis was performed on the briquettes.

\section{CHAPTER 3. STORAGE AND TRANSPORTATION OF CWF}

The EERC conducted an initial review of the selected CWF's handling properties. Specifically, the storage and transportation properties for given fuels were evaluated. Storage properties were evaluated using a rod penetrometer test which measures the static stability of the quasi-liquid fuel over time. Pumpability of the slurry fuels was determined based primarily on the rheological profile of the CWF.

\subsection{CWF Pumpability Review}

The theoretical flow behavior analysis of CWFs indicates that although substantial advances in the technical understanding have taken place in the past few years, the three non-Newtonian categories (power law, yield power law, and Bingham plastic) remain distinct. Non-Newtonian pipeline design methods and techniques which work with one rheological category cannot be applied to another. Laboratory test data are mandatory for credible pipeline design, because the rheological properties of a slurry prepared from any particular coal can be affected by even small concentrations of common contaminants. Theoretical analysis should be carried out, particularly in 


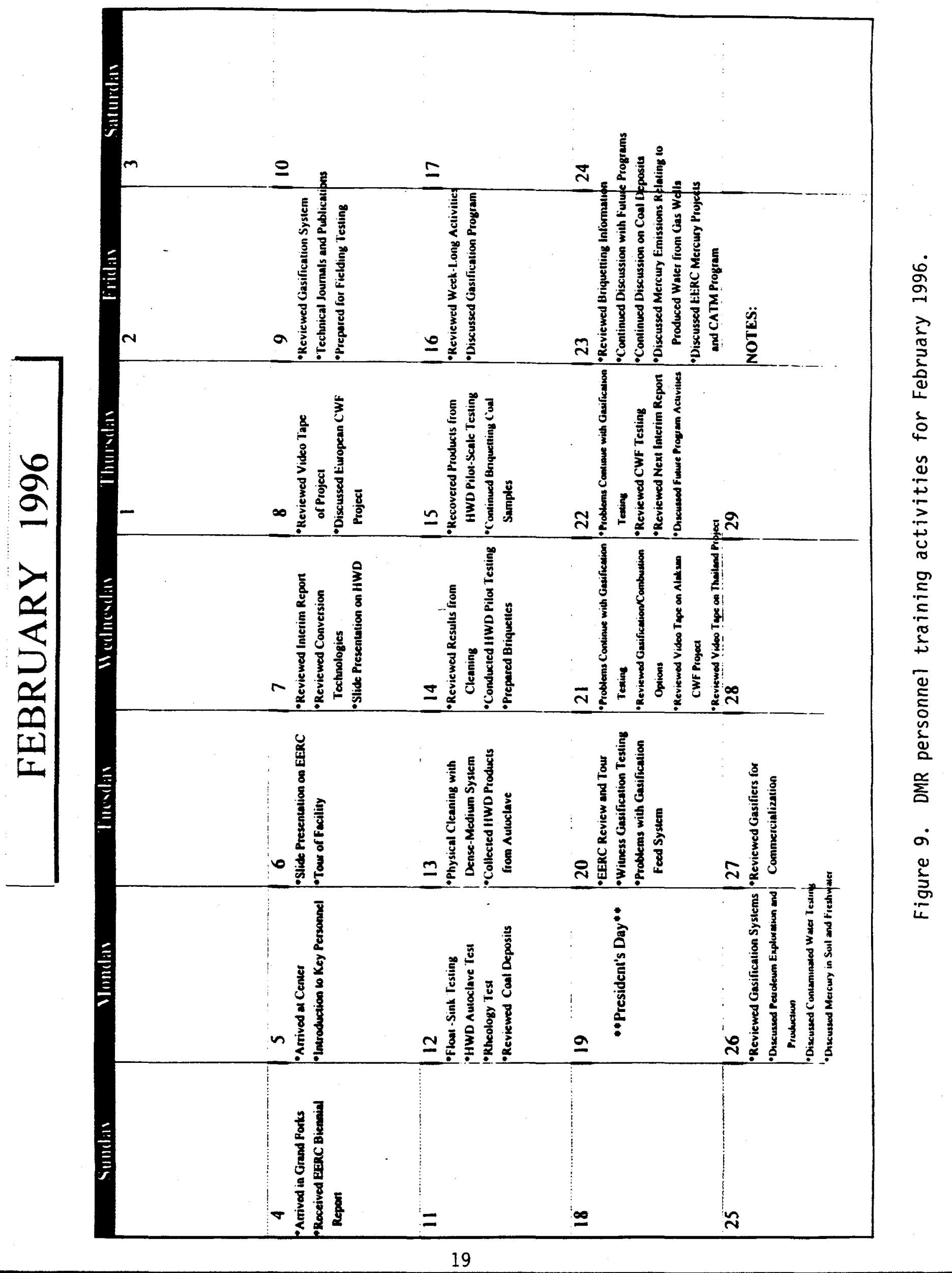


situations where an initial assessment is required. Identification and understanding of all variables and their impacts on the coal's stability and rheology are essential for developing design guidelines for transportation and utilization of the CWF.

Based on the rheological performance and computer simulation, pressure-drop analysis indicates that, for coal slurry pipeline transport, high-pressure positive displacement pumps may be used. The size and number of pump stations were determined using a computer program for nonNewtonian fluids developed at the EERC to analyze a frictional pressure drop. The pumping pressure requirements of the simulated slurry pipeline system were calculated from the following inputs: slurry flow rate, slurry solids loading, density, PSD, pipe size, distance of transport, and transport route. The program outputs included pump station power requirements and energy required for slurry transportation.

The pump station power requirements and energy requirements are presented in Figures 10 and 11 , respectively, for the raw, $275^{\circ}, 300^{\circ}$, and $325^{\circ} \mathrm{C}$ CWFs. The positioning of these pump stations was dependent upon the terrain crossed, the line size, and the economic balance between multiple pump stations and requirements for high-pressure design. Pump stations were spaced at 100 - to $150-\mathrm{km}$ intervals, which means that acceptable energy input requirements ranged from 15 to $30 \mathrm{~kW} /$ mile or 0.04 to $0.08 \mathrm{kWh} /$ ton-mile. At these energy requirements, the solids concentrations for the four fuels represented in Figures 10 and 11 are 36.1, 44.9, 47.9, and $48.8 \mathrm{wt} \%$ for the raw coal, $275^{\circ}, 300^{\circ}$, and $325^{\circ} \mathrm{C}$ HWD slurry fuels, respectively, at an approximate viscosity of $300 \mathrm{cP}$.

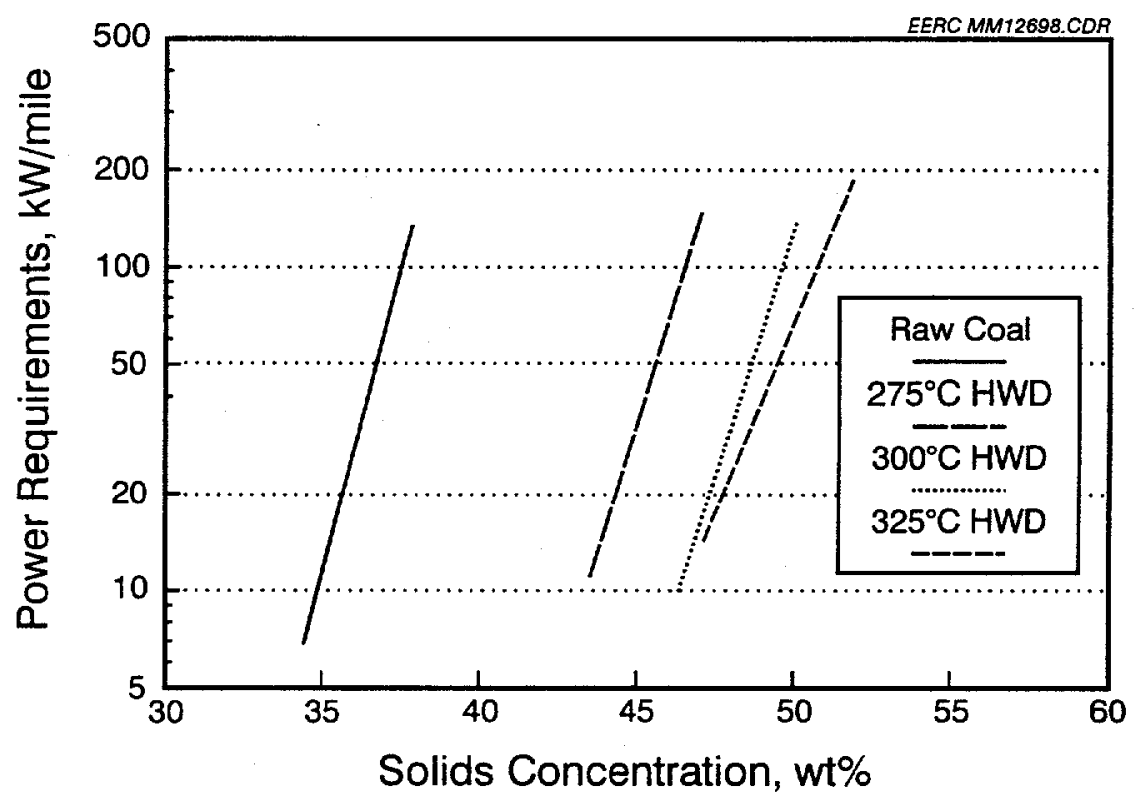

Figure 10. Pump requirements for transporting 3.5 MM tons/yr of Wiang Haeng coal CWF. 


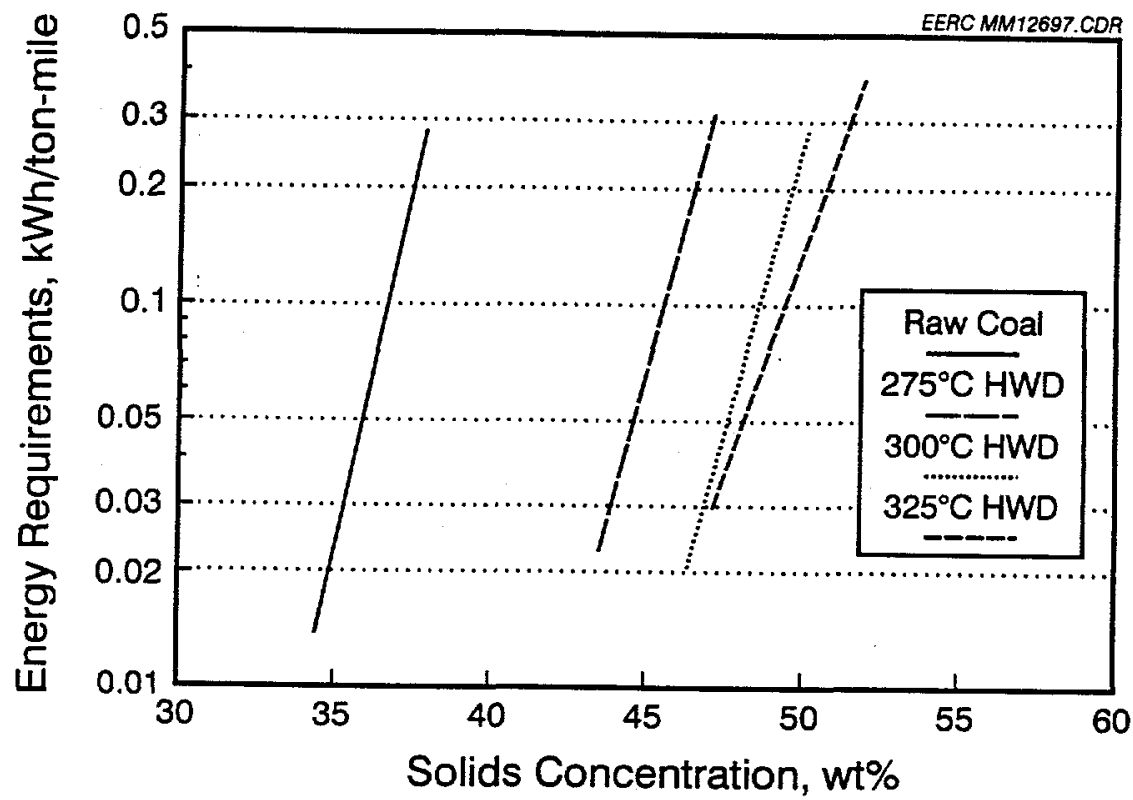

Figure 11. Energy requirements for transporting 3.5 MM tons/yr of Wiang Haeng coal CWF.

\subsection{CWF Stability Assessment}

A major thrust of the development work at the EERC has been to establish economically viable methods to improve the energy density of the CWF without relying on expensive additives to stabilize CWF. The enhanced technology is a proprietary EERC procedure applied either before or after HWD treatment which relies on mechanical means, not chemicals, to alter the coal surface to enhance the attainable solids by 3 to $5 \mathrm{wt} \%$, compared to just HWD. Figure 12 reflects the comparison of raw coal slurry to enhanced HWD processed fuel. The density, determined at $500 \mathrm{cP}$, indicates an increase from 4450 to $6650 \mathrm{Btu} / \mathrm{lb}$. In addition, the enhanced fuel, as indicated in Table 12, had stability properties far superior to those of the other fuels. Table 12 indicates the results from the rod penetrometer testing to determine the stability of the CWFs. Tabulated numbers represent the percentage relative to the distance that a rod of certain dimension travels through a coal slurry just after mixing and after various amounts of time: the lower the percentage, the more coal settling. In relation to the stability of the raw coal slurry, the HWD fuels appeared to be less stable over the same time period. More information on the instrument used to perform particle-size analysis and the rod penetrometer method is contained in Appendix A. This information is an initial evaluation of handling properties. To further understand CWF stability, a complete assessment of dynamic and static properties should be conducted using a pipeloop system and conventional storage methods. 


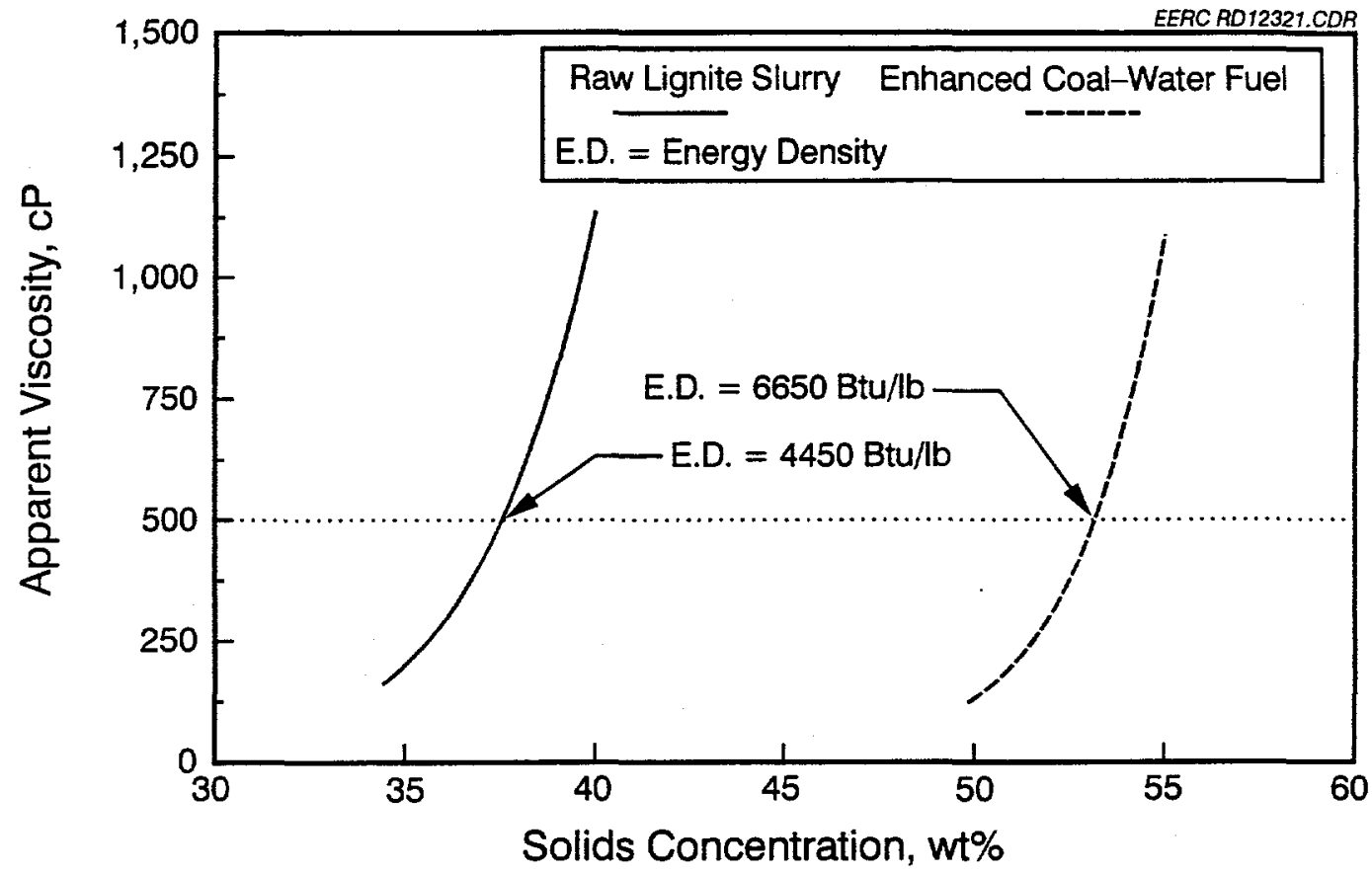

Figure 12. Rheological results for raw coal slurry and enhanced CWF.

TABLE 12

Rod Penetrometer Test Results on Raw and HWD Samples

\begin{tabular}{lcrrr}
\hline & \multicolumn{4}{c}{$\%$ Penetrated } \\
\cline { 2 - 5 } Time, hours & Raw & HWD, & HWD, & HWD, \\
\hline 1 & Coal & $300^{\circ} \mathrm{C}$ & $325^{\circ} \mathrm{C}$ & $325^{\circ} \mathrm{C} \mathrm{En}^{1}$ \\
5 & 97.4 & 100.0 & 91.7 & 100.0 \\
8 & 89.5 & 77.1 & 61.1 & 86.1 \\
24 & 89.5 & 62.9 & 44.4 & 86.1 \\
29 & 73.7 & 40.0 & 33.3 & 61.1 \\
31 & 71.1 & 5.7 & 0.0 & 5.6 \\
48 & 57.9 & 0.0 & 0.0 & 0.0 \\
\hline
\end{tabular}

${ }^{1}$ Enhanced CWF.

\section{CHAPTER 4. CONCEPTUALIZED CWF DEVELOPMENT PLAN}

Initial efforts by the EERC and DMR have focused on the hot-water-drying technology and its immediate applications. No research has been conducted on the potential site, economics, or 
marketability of the products. If the coal quality and product specifications are achieved, the next stage of development would be to complete a process review for a specific site; this includes the following:

- Evaluate site and coal reserves

- Establish coal processing capacities based on available raw materials and utilities

- Develop process flow diagram for selected site

- Conduct process economic review

- Review market potential for CWF

After these results are compiled and if the indications are still favorable, developers then need to consider a process demonstration system. This intermediate stage will allow individuals to review process conditions and refine economic and market projections. The EERC offers technical assistance for nearly all aspects of process development. Its role in future development may include technical oversight and technology transfer. Technology transfer is a critical component to the successful project for advancing countries such as Thailand. The EERC has completed numerous training courses for new technologies and has critical insight into the fabrication and operation of hot-water-drying systems and CWF formulation. As technology developers, the EERC will provide input to the selected contractors for the design, fabrication, shakedown, and operation of future demonstration or commercial facilities.

\section{CHAPTER 5. CONCLUSIONS}

- Approximately $600 \mathrm{~kg}$ of Wiang Haeng coal was received at the EERC for testing.

- HWD enhanced the energy density of a pumpable Wiang Haeng coal slurry by $50 \%$.

- Approximately $250 \mathrm{~kg}$ of HWD coal-water fuel was successfully produced using pilot-scale system.

- The existing EERC bench-scale gasification system had difficulties feeding finer grind coal.

- Gasification tests were successfully completed using larger size $(6.35 \times 00 \mathrm{~mm})$ raw and HWD coal.

- Results from gasification tests indicated gas production was approximately $30 \%$ of the feed, with hydrogen production accounting for over $50 \%$ of the total gas production.

\section{CHAPTER 6. RECOMMENDATIONS}

Based on the success of CWF experiments, the next stages of development may include testing new coals or testing CWF in a combustion system. Also, additional data are required to evaluate the storage and transportation properties of the CWF. Continued success may lead to extended pilot-scale testing and eventually to commercial demonstration in Thailand. 
Specific tasks the EERC and DMR may consider for future gasification program development include the need to define products for specific gasifier types: slurry fuel, solid fuel product (briquettes, fine char), synfuel, or electrical production. Future product testing may include an evaluation of the amount of gas, char, and tar produced at various temperature conditions. Bench-scale tests would then be conducted to optimize tar production versus volatile content of the char. In order to consider synfuel and electrical production opportunities, laboratory tests may include thermogravimetric analysis matrix testing to investigate steam reactivity. Also, a more thorough ash characterization would be needed to assess slagging characteristics. 
Energy \&

Environmental

Research

Center

APPENDIX A

ANALYTICAL EQUIPMENT AND PROCEDURES 


\section{ANALYTICAL EQUIPMENT AND PROCEDURES}

The Energy \& Environmental Research Center (EERC) has researched numerous drying and conversion technologies that can be applied to coal and subbituminous coals to upgrade the quality of the coal. Table A-1 summarizes the test procedures used to analyze the coal samples for this project.

\section{TABLE A-1}

Analytical Equipment and Procedures

\begin{tabular}{|c|c|c|}
\hline Analysis & Equipment & Procedure \\
\hline Hot-Water Drying & Two-Gallon Autoclave & $\mathrm{NA}^{1}$ \\
\hline Fuel Viscosity & Haake $^{\circledR} 100$ Rotoviscometer & NA \\
\hline Static Stability & Rod Penetrometer & \\
\hline Particle Size & Malvern ${ }^{\circledR} 2600 c^{c}$ Laser Diffraction & NA \\
\hline Gas Analysis & Hewlett-Packard ${ }^{\otimes} 5880 \mathrm{a}$ Gas & \\
\hline Carbon & Leco $^{\circledR}$ CHN 600 Analyzer & NA \\
\hline Hydrogen & Leco ${ }^{\otimes}$ CHN 600 Analyzer & NA \\
\hline Nitrogen & Leco $^{\circledR}$ CHN 600 Analyzer & NA \\
\hline Sulfur & Leco $^{\otimes}$ Induction Furnace & ASTM D3177 \\
\hline Sulfur Forms & NA & ASTM D2492 \\
\hline Equilibrium Moisture & NA & ASTM D1412 \\
\hline Moisture & Fisher ${ }^{\circledR}$ Coal Analyzer & ASTM D3175 \\
\hline Volatile Matter & Fisher ${ }^{\circledR}$ Coal Analyzer & ASTM D3175 \\
\hline Ash & Fisher $^{\circledR}$ Coal Analyzer & ASTM D3174 \\
\hline Ash Characterization & Kevex ${ }^{\circledR}$ Energy-Dispersive & \\
\hline Combustion Behavior & Drop-Tube Furnace (DTF) & NA \\
\hline
\end{tabular}

${ }^{1}$ Not applicable.

\section{PROXIMATE AND ULTIMATE ANALYSIS}

Proximate analysis (moisture, ash, volatile matter, fixed carbon) was performed on the raw and treated coal using a Fisher ${ }^{\circledR}$ coal analyzer. The instrument determines moisture at conditions specified in American Society for Testing and Materials (ASTM) Method D3175. Volatile matter 
is determined by heating the dried sample to $950^{\circ} \mathrm{C}$ in a nitrogen atmosphere at conditions specified in ASTM D3175. The sample is covered during this process in order to exclude oxygen and prevent the sample from being ashed. Following the volatile matter determination, the sample is uncovered and combusted, and the ash is determined at conditions specified by ASTM D3174. Fixed carbon is determined by difference, following the previously described methods.

Ultimate analysis determines carbon, hydrogen, nitrogen, sulfur, oxygen, and ash content in a sample. Carbon, hydrogen, and nitrogen (CHN) content are determined using a Leco ${ }^{\circ} \mathrm{CHN}-600$ analyzer. Carbon and hydrogen are determined by infrared cells, and elemental nitrogen is measured by a thermal conductivity cell. This method gives the total percentages of CHN in the organic sample as analyzed and includes the carbon in carbonates and the hydrogen in the moisture and in the water of hydration of minerals. Sulfur is determined using a Leco ${ }^{\oplus}$ SC-132 sulfur analyzer in which a sample is combusted in oxygen, forming sulfur dioxide, which is determined by an infrared cell detector. Ash is determined by the ASTM method described under proximate analysis. Oxygen is determined by difference in order to achieve a balance of the ultimate analysis. Forms of sulfur are determined according to ASTM D2492. Ash characterization is determined using $\mathrm{x}$-ray fluorescence techniques using the equipment in Table A-1.

\section{RHEOLOGICAL CHARACTERIZATION}

The rheological properties are determined based on the Yield Power Law equation for fluids. The Haake ${ }^{\otimes V} 100$ viscometer, shown in Figure A-1, is used to measure the flow properties of various liquid fuels from shear rates of near zero to $1100 \mathrm{sec}^{-1}(\mathrm{~Hz})$ over a temperature range of $4^{\circ}$ to $95^{\circ} \mathrm{C}$. Results from the Haake are logged and compiled using a computer network system. annular space between the rotating cylinder and a stationary cup. The torque necessary to rotate the cylinder at a given speed is measured by a torsion spring. Various rotor assemblies allow the

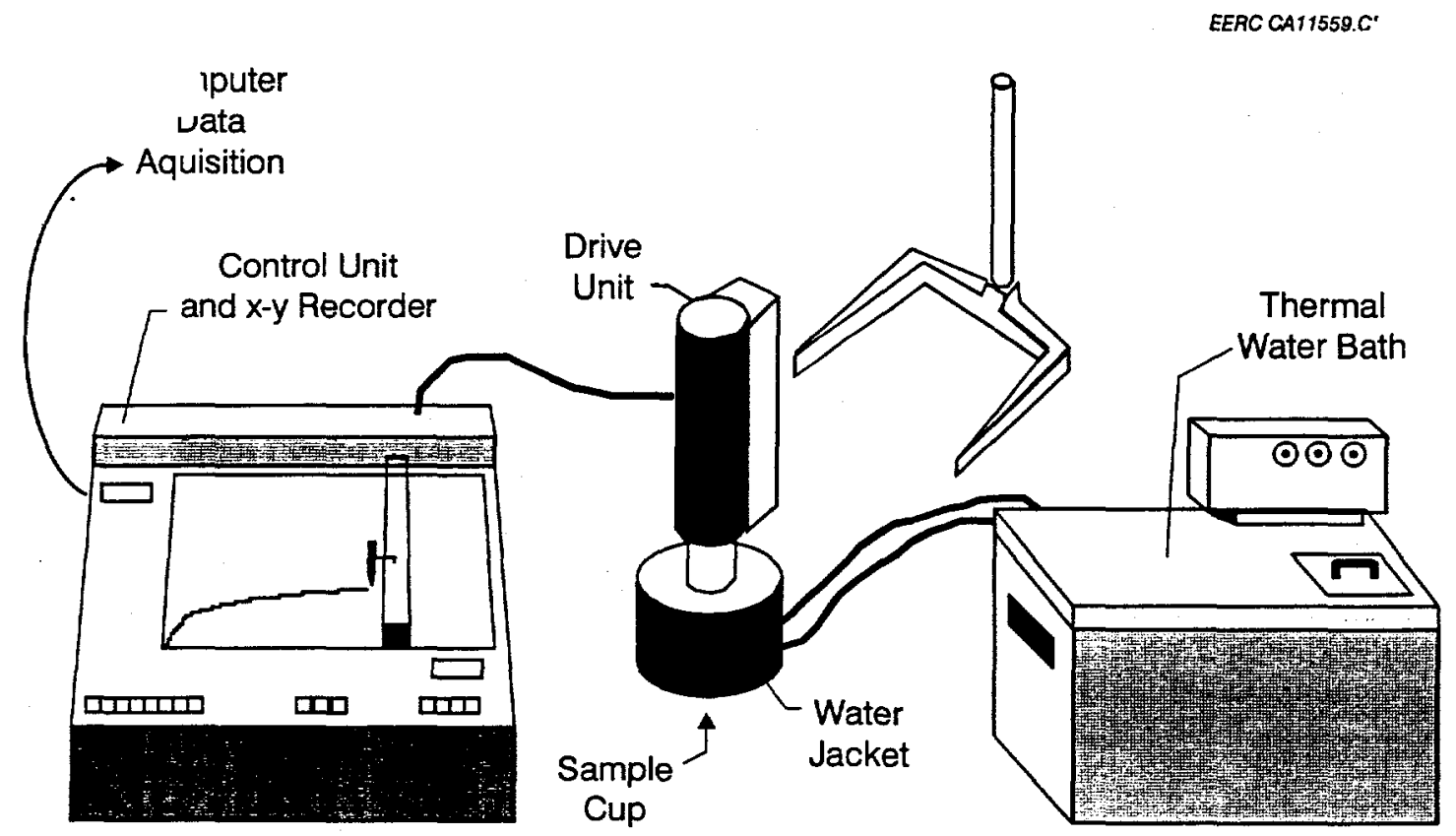

Figure A-1. $\mathrm{HAAKE}^{\otimes}$ rheological measuring equipment. 
The computer indicates the independent flow behavior, viscosity, and yield stress values for the fluid as each correlates to the Yield Power Law. The Haake ${ }^{\oplus i s c o m e t e r ~ s h e a r s ~ t h e ~ s l u r r y ~ i n ~ t h e ~}$ user a complete profile of the rheological characteristics. Rheological data are used to assess the effect of the hot-water drying (HWD) process variables on the relationship between viscosity and dry solids concentrations.

\section{PARTICLE SIZE}

The particle-size distribution of the raw and HWD coal is determined using a Malvern ${ }^{8}$ $2600 \mathrm{c}$ laser diffraction particle-size analyzer, capable of measuring particle sizes from 0.5 to 564 microns. Figure A-2 depicts the Malvern ${ }^{\circledR}$ instrument setup. The basic principle of the Malvern involves a He-Ne low-power visible wavelength laser that is first expanded and spatially filtered to provide a clean parallel beam. As particles pass through the beam, they scatter or diffract the light at different angles, depending upon their diameter; large particles scatter at small angles and vice versa. The scattered light is collected by a lens and brought to focus on a multielement solid-state detector that simultaneously measures the light at a number of angles. During analysis, the sample particles move rapidly through the laser beam. The results indicate the volume percentage distribution, as well as report the average particle size.

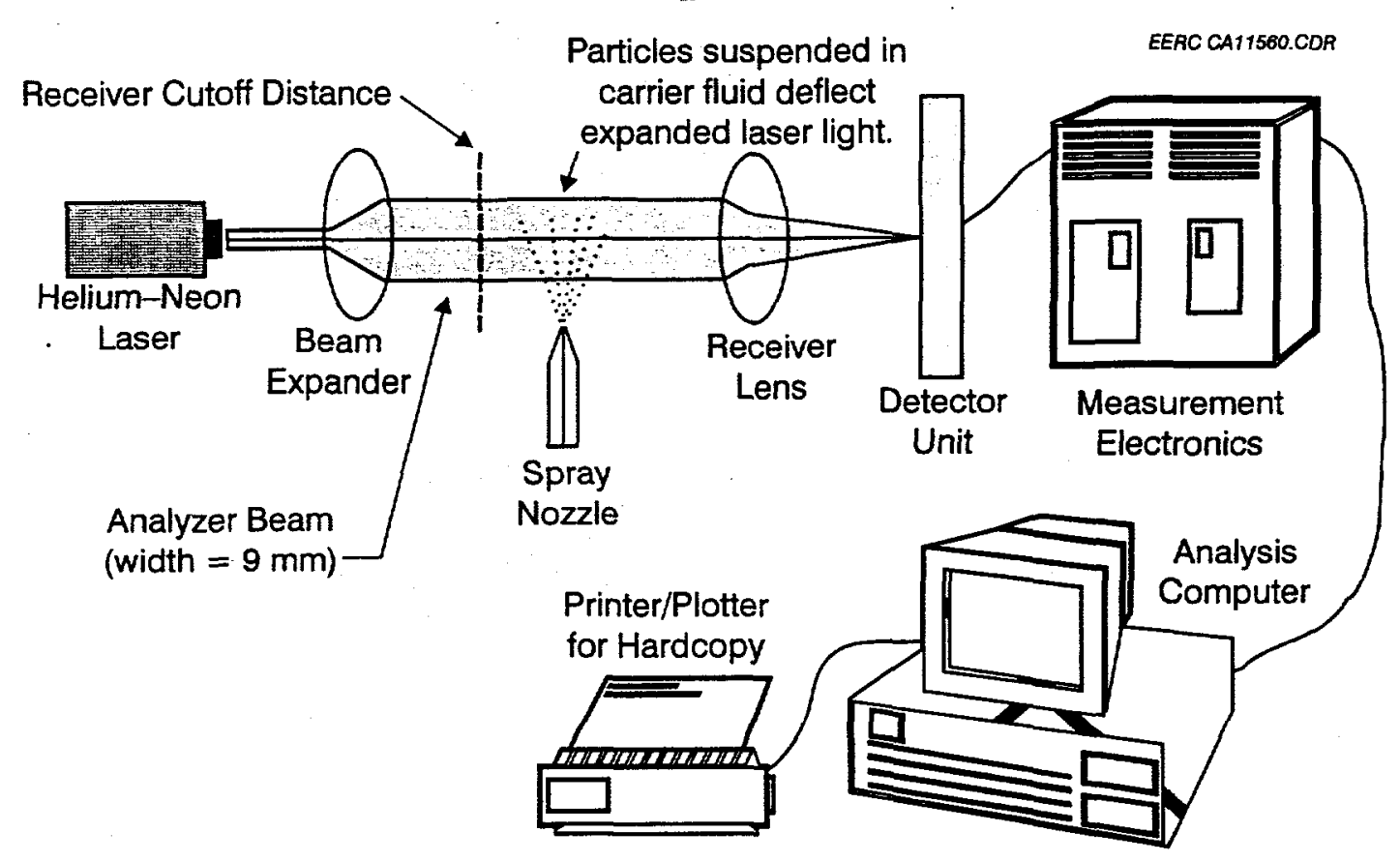

Figure A-2. Malvern ${ }^{\oplus}$ instrument setup. 


\section{ROD PENETRATION TEST}

The rod penetration test is a static stability method that was developed for coal-liquid slurries. It is based on the distance a glass rod travels through the slurry in comparison to the maximum distance through the slurry. The method involves suspending the glass rod above the slurry and then slowly lowering into the slurry until the rod stops moving. Then a visual measurement using a level and a ruler is taken to determine the amount of penetration. Penetration was calculated according to the following equation:

$$
\% \text { Penetration }=\mathrm{d} / \mathrm{d}_{\mathrm{T}} \times 100
$$

where:

$$
\begin{aligned}
& \mathrm{d}=\text { distance of rod travel }(\mathrm{cm}) \\
& \mathrm{d}=\mathrm{d}_{\mathrm{t}}=\text { maximum distance of rod travel }(\mathrm{cm})
\end{aligned}
$$

\section{EQUTLIBRIUM MOISTURE}

The moisture contents of the raw and HWD coals as a function of incubation time are determined using a standard or modified ASTM equilibrium moisture method. The standard equilibrium moisture tests use an incubation time of 3 or 4 days, while the modified test uses incubation times of 2 to 30 days. The extended time period is used because the standard 3 or 4 days is often too short to allow the raw and HWD low-rank coals to attain an equilibrium moisture.

\section{PROCESS WATER ANALYSIS}

Various tests are performed on the process water produced during HWD to evaluate the amount of carbonaceous material, the recyclability of the stream, and treatability. The most widely used parameter of organic pollution applied to both wastewater and surface water is the 5-day biochemical oxygen demand (BOD) test. The BOD test is completed on the water sample to measure the oxygen required for the biochemical degradation of organic material and the oxygen used to oxidize inorganic material. The BOD is computed from the difference between initial and final dissolved oxygen. The chemical oxygen demand (COD) test is used to measure the content of organic matter of both wastewater and natural waters. The COD of a waste is, in general, higher than the BOD because more compounds can be chemically oxidized than can be biologically oxidized. For many types of wastes, it is possible to correlate COD with BOD. This can be very useful because the COD can be determined in 3 hours, compared to 5 days for the BOD. Once the correlation has been established, COD measurements can be used to good advantage for treatment plant control and operation. Total organic carbon (TOC), another means for measuring the organic matter present in water, is especially applicable to small concentrations of organic matter. To determine the quantity of TOC, the organic molecules must be broken down to single carbon units and converted to a single molecular form that can be measured quantitatively. TOC methods utilize heat and oxygen, ultraviolet irradiation, chemical oxidants, or combinations of these oxidants to convert organic carbon to carbon dioxide. Total carbon (TC) is determined by taking a quantity of sample and analyzing directly using an infrared carbon analyzer. In order to determine TOC, the 
sample must be sparged of the inorganic carbon (IC). Total solids determination is also performed on the sample. This includes total dissolved solids (TDS) and total suspended solids (TSS). TSS includes the portion of total solids retained by a filter, and TDS is the portion of the total solids that passes through the filter.

\section{GAS ANALYSIS}

Gas analysis is evaluated using a Hewlett-Packard ${ }^{\circledR}$ Model 5880a gas chromatograph with a refinery gas analyzer package used to detect selected gases in process gas samples. The method of detection is based upon relationships of thermal conductivity of the various gas compounds. The refinery package consists of five different absorbing columns and four heated valves for column switching. The instrument uses two carrier gases, and hydrogen is used for the determination of $\mathrm{CO}_{2}, \mathrm{CO}, \mathrm{O}_{2}, \mathrm{~N}_{2}, \mathrm{H}_{2} \mathrm{~S}$, and carbon chains $\mathrm{C}_{1}$ through $\mathrm{C}_{5}$ hydrocarbons. Argon is used for the determination of helium and hydrogen.

\section{WIANG HAENG COAL-WATER FUEL PROGRAM}

A total of six tests were performed; variables evaluated included particle-size distribution and treatment temperature. The HWD-treated solids were then reslurried in water and evaluated for size distribution and rheological performance. The particle-size distribution is important, because if the particles are too large, there will be more void spaces and lower packing efficiency, resulting in a decreased fuel solids concentration. The rheological behavior for the coal-liquid mixture, specifically the apparent viscosity, was determined as a function of shear rate and CWF solids concentrations. Since any change in solids loading of a coal-water fuel has a direct effect on the resulting flow behavior, several theograms throughout the possible solids loading range were gathered. Table A-2 summarizes the characterization protocol for products of hydrothermal treatment. 
TABLE A-2

Analysis Protocol for Products of Wiang Haeng coal Hydrothermal Treatment

\begin{tabular}{|c|c|c|c|}
\hline Analysis & $275^{\circ} \mathrm{C}$ & $300^{\circ} \mathrm{C}$ & $325^{\circ} \mathrm{C}$ \\
\hline \multicolumn{4}{|l|}{ Filter Cake } \\
\hline Proximate & $\checkmark$ & $\checkmark$ & $\checkmark$ \\
\hline Ultimate & $\checkmark$ & $\checkmark$ & $\checkmark$ \\
\hline Heating Value & $\checkmark$ & $\checkmark$ & $\checkmark$ \\
\hline Ash Chemistry & $\checkmark$ & $\checkmark$ & $\checkmark$ \\
\hline Ash Fusion & & $\checkmark$ & $\checkmark$ \\
\hline Particle Size & $\checkmark$ & $\checkmark$ & $\checkmark$ \\
\hline \multicolumn{4}{|l|}{ Slurry Fuel } \\
\hline Rheology & $\checkmark$ & $\checkmark$ & $\checkmark$ \\
\hline Stability & $\checkmark$ & $\checkmark$ & $\checkmark$ \\
\hline \multicolumn{4}{|l|}{ Process Water } \\
\hline TDS & $\checkmark$ & $\checkmark$ & $\checkmark$ \\
\hline TSS & $\checkmark$ & $\checkmark$ & $\checkmark$ \\
\hline COD & $\checkmark$ & $\checkmark$ & $\checkmark$ \\
\hline BOD & $\checkmark$ & $\checkmark$ & $\checkmark$ \\
\hline TOC & $\checkmark$ & $\checkmark$ & $\checkmark$ \\
\hline TC & $\checkmark$ & $\checkmark$ & $\checkmark$ \\
\hline \multicolumn{4}{|l|}{ Process Gas } \\
\hline Composition & $\checkmark$ & $\checkmark$ & $\checkmark$ \\
\hline Molecular Weight & $\checkmark$ & $\checkmark$ & $\checkmark$ \\
\hline Specific Gravity & $\checkmark$ & $\checkmark$ & $\checkmark$ \\
\hline
\end{tabular}


Energy $\mathcal{E}$

Environmental

Research

Center

APPENDIX B

AUTOCLAVE TEMPERATURE
AND PRESSURE TRENDS 


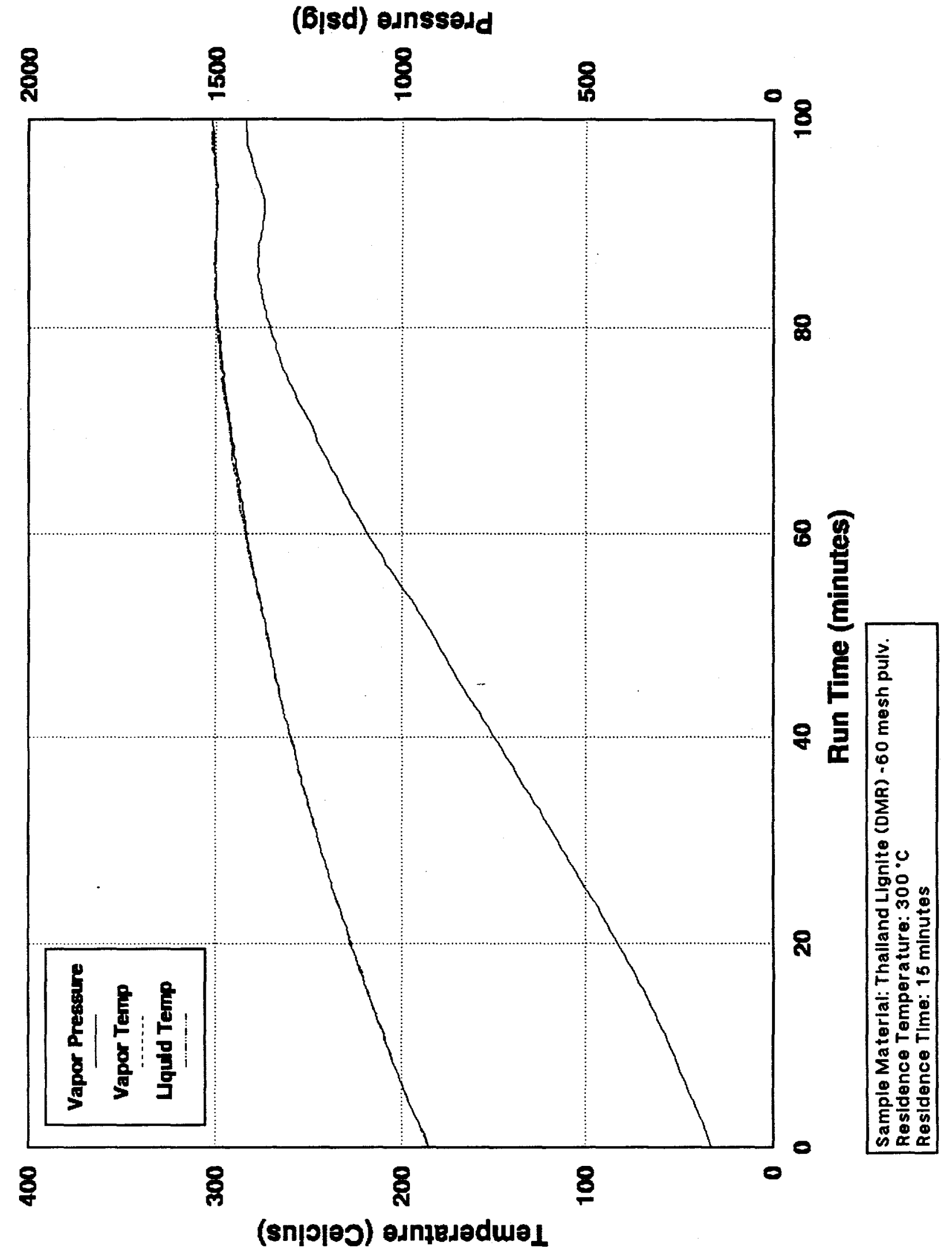


(bisd) odnssedd

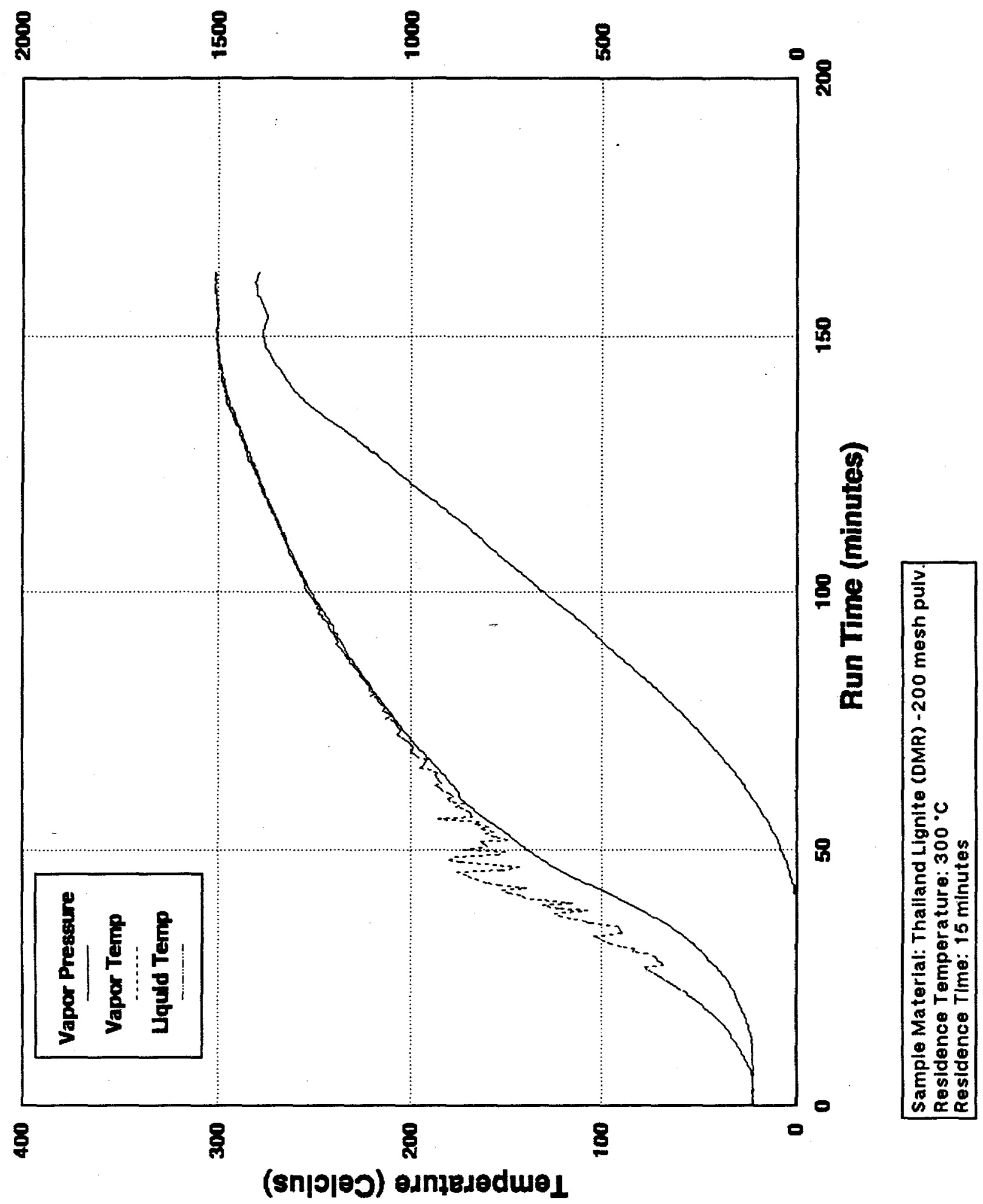


(Bjsd) odnssedd

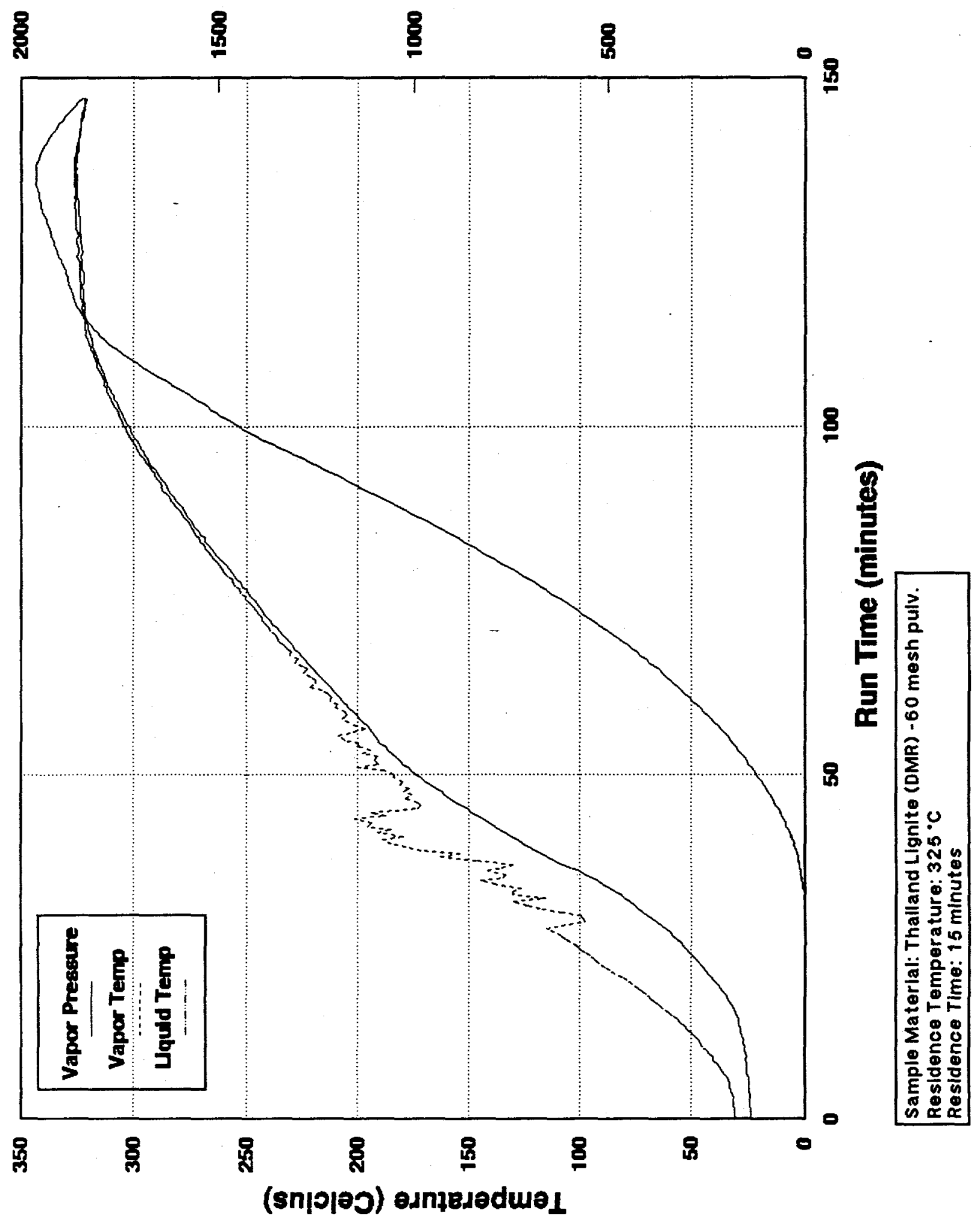


(6jsd) anns8edd

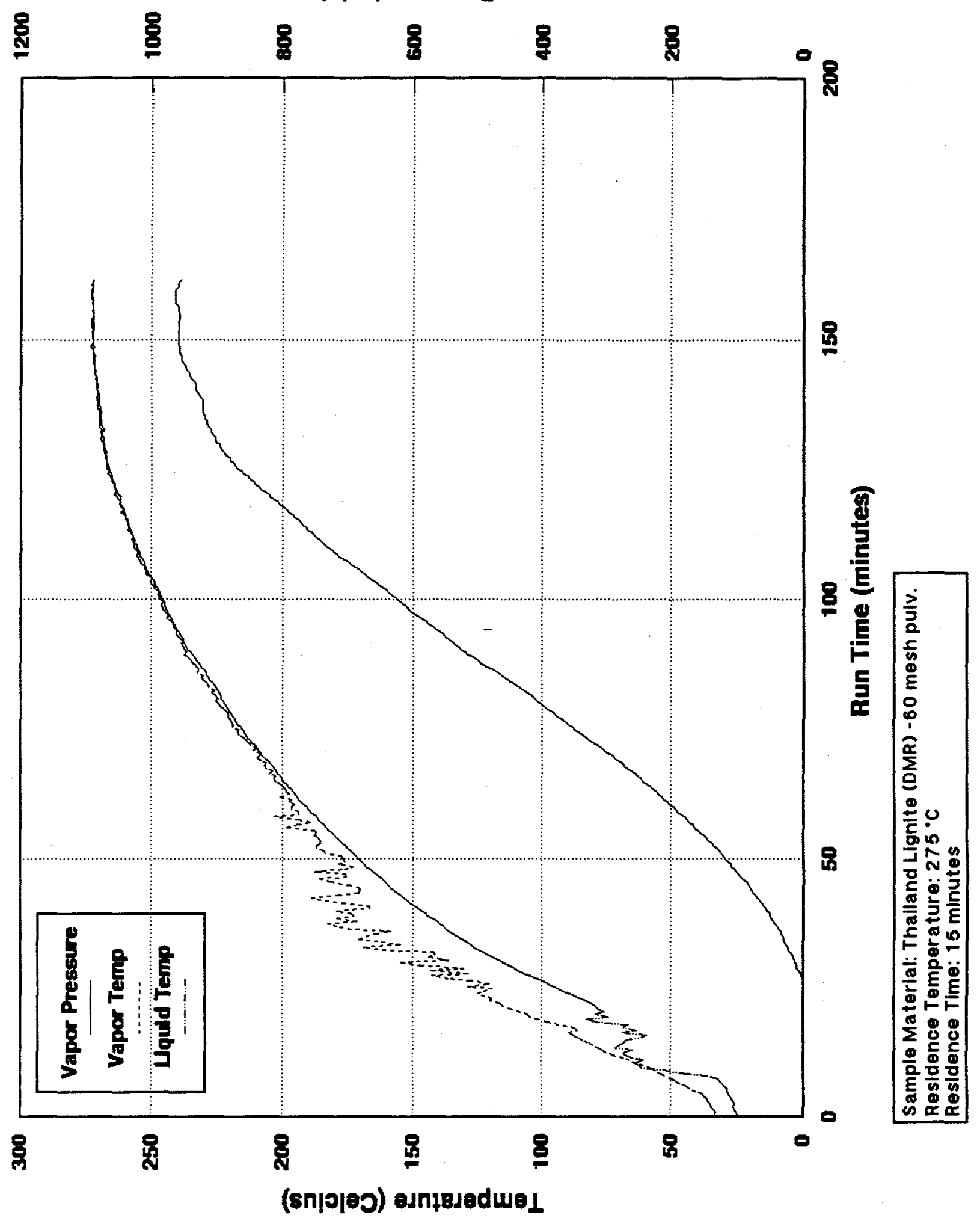




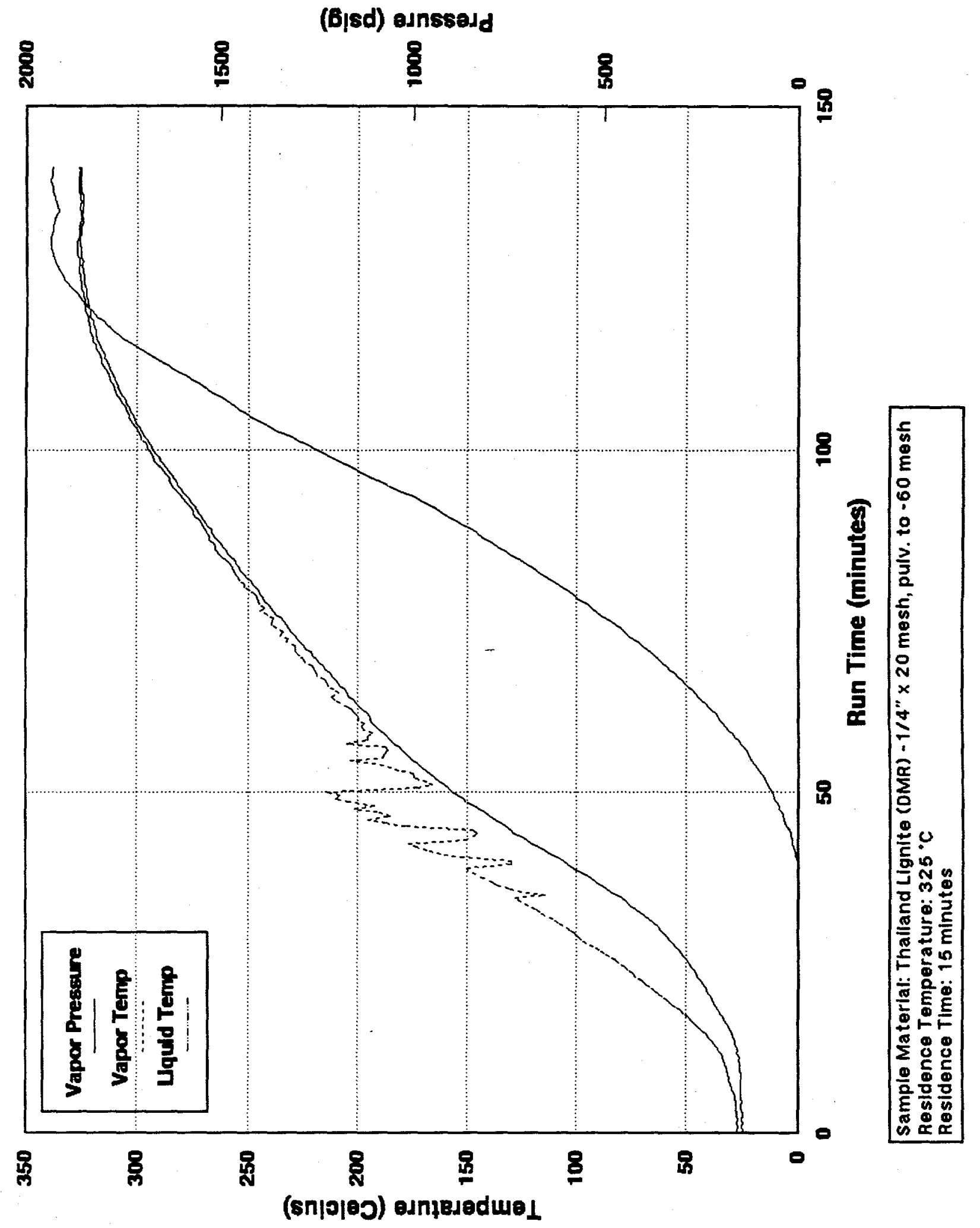




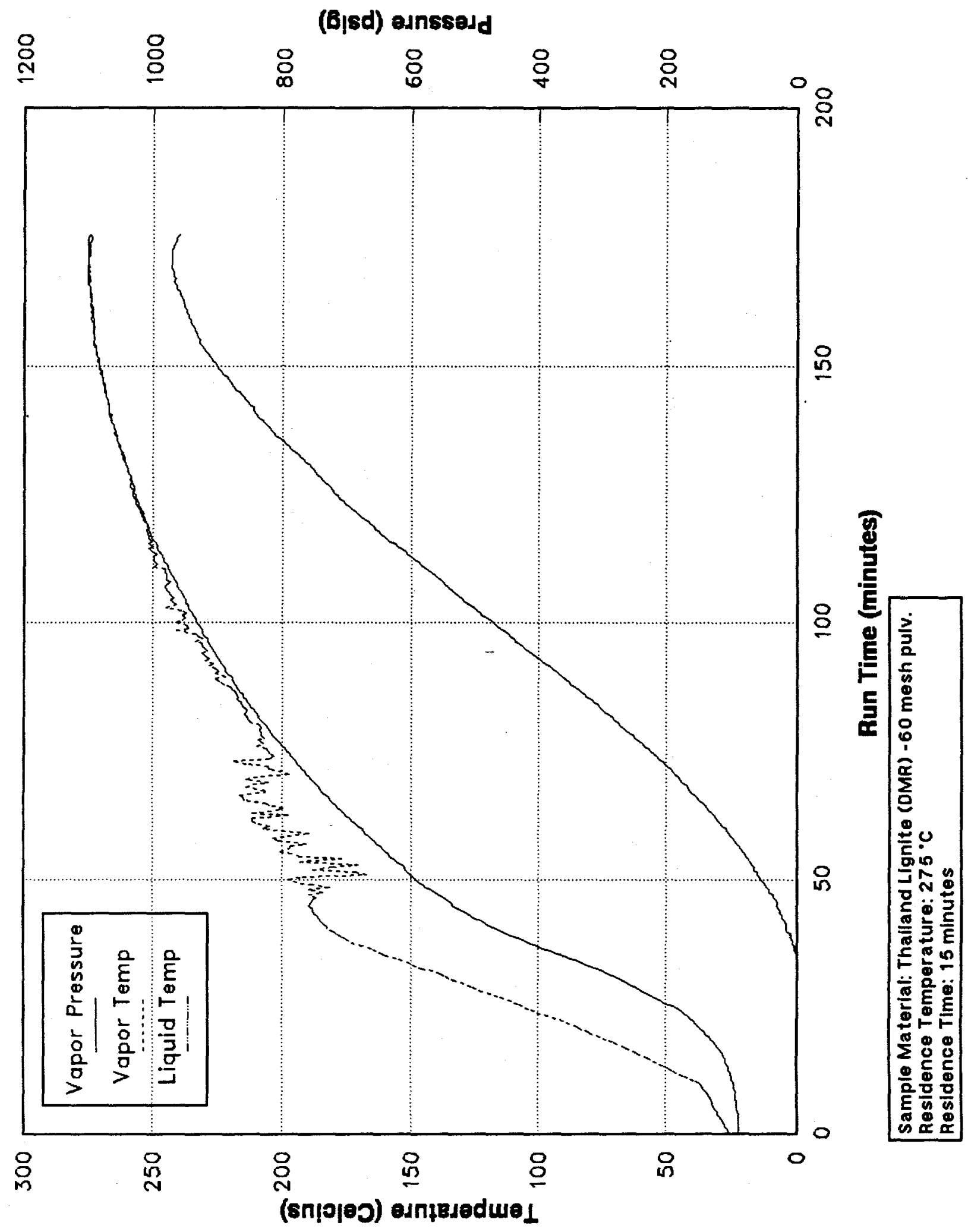




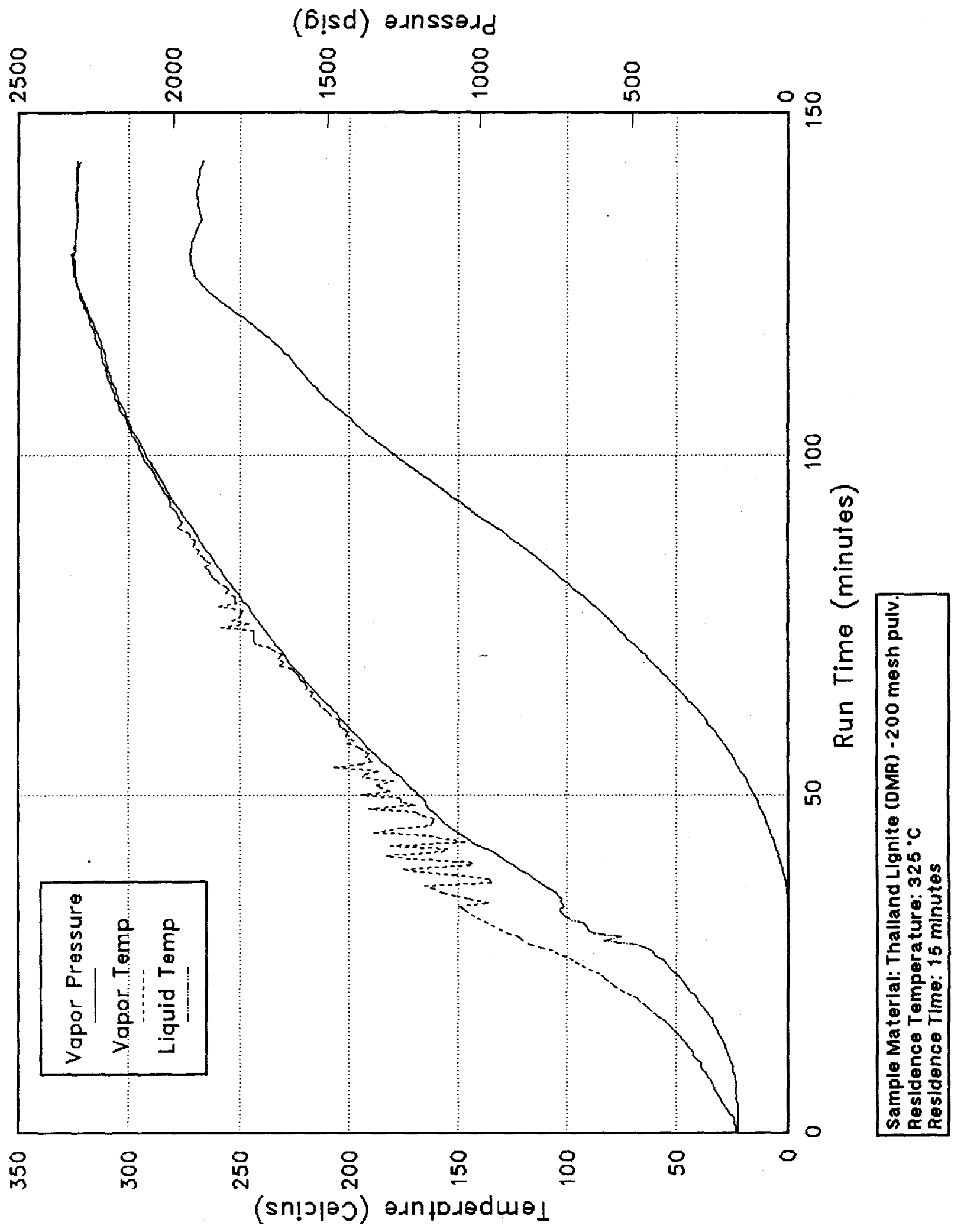


Energy \&

Environmental

Research

Center

\section{APPENDIX C}

\section{RHEOLOGICAL PROFILES FOR COAL-WATER FUELS}


ENERGY \& ENVIRONMENTAL RESEARCH CENTER

COAL BENEFICIATION PROJECT

Grand Forks, North Dakota 58202

\begin{tabular}{|c|c|c|c|}
\hline \multicolumn{4}{|c|}{$\begin{array}{l}\text { RHEOLOGY FOR THAILAND AUTOCLAVE SAMPL } \\
\text { TEST\#: THAI. O3 HWD @ } 325 \mathrm{C}(-60 \text { mesh } \\
\text { LOW SOLIDS CONCENTRATION }\end{array}$} \\
\hline DATE > NOV & \multicolumn{2}{|c|}{ TIME > $08: 41: 15$} & \\
\hline \multicolumn{4}{|c|}{ ARCHIVE FILE NAME >c: $\backslash 123 \mathrm{r} 3 \backslash \mathrm{THO} 34983 . \mathrm{p}$} \\
\hline \multicolumn{4}{|c|}{ SOLIDS CONCENTRATION } \\
\hline Separate & 49.82 & Wt & \\
\hline Average & & Ht & \\
\hline
\end{tabular}

\begin{tabular}{|l|c|c|c|}
\hline \multicolumn{3}{|c|}{ ASH CONCENTRATION } \\
\hline Separate & $* * * * *$ & $* * * * *$ & ; t $\%$ \\
\hline Averase & \multicolumn{2}{|c|}{$* * * * *$} & Wt $\%$ \\
\hline
\end{tabular}

\begin{tabular}{|l|c|l|}
\hline \multicolumn{3}{|c|}{ VISCOMETER CONFIGURATION } \\
\hline Sensor & MV-IIP & Sensor Type \\
\hline Eactor A & 3.76 & Pascals \\
\hline Eactor $M$ & 4.40 & $1 /$ sec \\
\hline Temperature & 25.00 & 'Celcius \\
\hline$\because 5$ & 100.00 & Percent (\%) \\
\hline$\%$ Tau & 20.00 & Percent (\%) \\
\hline
\end{tabular}

\begin{tabular}{|c|c|}
\hline $\begin{array}{c}\text { SHEAR } \\
\text { RATE }\end{array}$ & $\begin{array}{l}\text { APPARENT } \\
\text { VISCOSITY }\end{array}$ \\
\hline$+50 \cdot \mathrm{Hz}$ & $99.52 \cdot \mathrm{cP}$ \\
\hline$+100 \cdot \mathrm{Hz}$ & $93.84 \cdot \mathrm{cP}$ \\
\hline$+200 \cdot \mathrm{Hz}$ & $84.04 \cdot \mathrm{cP}$ \\
\hline$+300 \cdot \mathrm{Hz}$ & $81.07 \cdot \mathrm{cP}$ \\
\hline$+400 \cdot \mathrm{Hz}$ & $80.38 \cdot \mathrm{cP}$ \\
\hline$-400 \cdot \mathrm{Hz}$ & $83.95 \cdot \mathrm{cP}$ \\
\hline$-300 \cdot \mathrm{Hz}$ & $89.96 \cdot \mathrm{cP}$ \\
\hline$-200 \cdot \mathrm{Hz}$ & $99.92 \cdot \mathrm{cP}$ \\
\hline$-100 \cdot \mathrm{Hz}$ & $116.17 \cdot \mathrm{cP}$ \\
\hline$-50 \cdot \mathrm{Hz}$ & $147.02 \cdot \mathrm{cP}$ \\
\hline
\end{tabular}

\begin{tabular}{|l|c|c|l|}
\hline RHEOLOGICAL CLASSIFICATION & \multicolumn{4}{|l|}{ PSEUDOPLASTIC } \\
\hline INITIAL YIELD STRESS & $T$ & 0.2962 & Pascals \\
\hline CONSISTENCY FACTOR & $\mathrm{K}$ & 0.2053 & Pascal-Sec \\
\hline NON-NENTONIAN FLOW FACTOR & $n$ & 0.8346 & Dimensionless \\
\hline CURVE FIT ACCURACY & $\mathrm{R}^{2}$ & 99.9133 & Percent(\%) \\
\hline
\end{tabular}


ENERGY \& ENVIRONMENTAL RESEARCH CENTER

COAL BENEFICIATION PROJECT

Grand Forks, North Dakota 58202

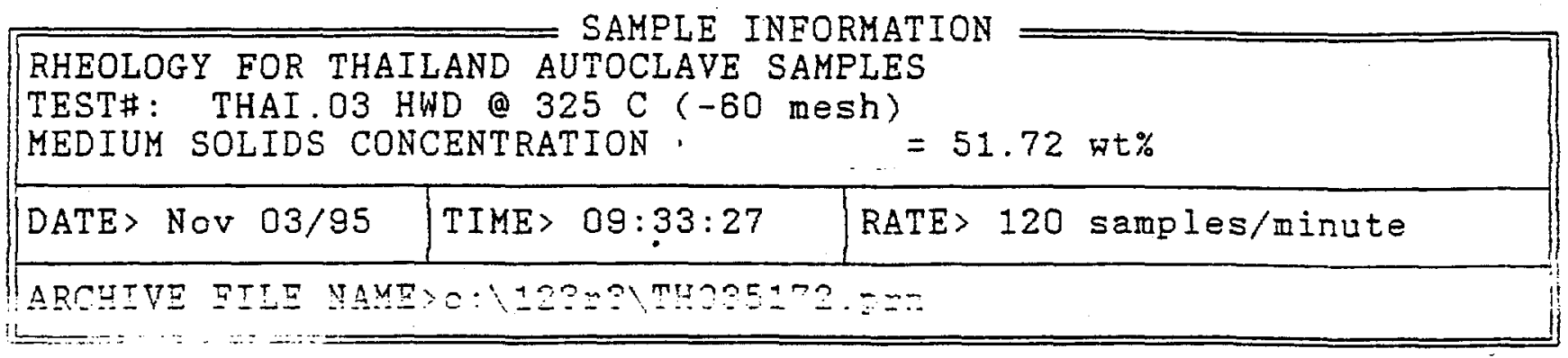

\begin{tabular}{|c|c|c|c|}
\hline \multicolumn{4}{|c|}{ SOLIDS CONCENTRATION } \\
\hline Separate & 51.71 & 51.73 & Wt \% \\
\hline Average & \multicolumn{2}{|c|}{51.72} & Ht \% \\
\hline
\end{tabular}

\begin{tabular}{|l|c|c|c|}
\hline \multicolumn{3}{|c|}{ ASH CONCENTRATION } \\
\hline Separate & $* * * * *$ & $* * * * * *$ & Wt \% \\
\hline Average & ******** & Wt \% \\
\hline
\end{tabular}

\begin{tabular}{|l|c|l|}
\hline \multicolumn{3}{|c|}{ VISCOHETER CONFIGURATION } \\
\hline Sensor & MV-IIP & Sensor Type \\
\hline Factor A & 3.76 & Pascals \\
\hline Eactor M & 4.40 & $1 /$ sec \\
\hline Temperature & 25.00 & ${ }^{\circ}$ Celcius \\
\hline$\%$ D & 100.00 & Percent (\%) \\
\hline$\%$ Tau & 40.00 & Percent (\%) \\
\hline
\end{tabular}

\begin{tabular}{|c|c|}
\hline $\begin{array}{c}\text { SHEAR } \\
\text { RATE }\end{array}$ & $\begin{array}{l}\text { APPARENT } \\
\text { VISCOSITY }\end{array}$ \\
\hline$+50 \cdot \mathrm{Hz}$ & $340.62 \cdot \mathrm{cP}$ \\
\hline$+100 \cdot \mathrm{Hz}$ & $280.73 \cdot \mathrm{cP}$ \\
\hline$+200 \cdot \mathrm{Hz}$ & $232.59 \cdot \mathrm{cP}$ \\
\hline$+300 \cdot \mathrm{Hz}$ & $219.52 \cdot \mathrm{cP}$ \\
\hline$+400 \cdot \mathrm{Hz}$ & $207.09 \cdot \mathrm{cP}$ \\
\hline$-400 \cdot \mathrm{Hz}$ & $215.27 \cdot \mathrm{cP}$ \\
\hline$-300 \cdot \mathrm{Hz}$ & $223.22 \cdot \mathrm{cP}$ \\
\hline$-200 \cdot \mathrm{Hz}$ & $245.01 \cdot \mathrm{cP}$ \\
\hline$-100 \cdot \mathrm{Hz}$ & $303.45 \cdot \mathrm{cP}$ \\
\hline$-50 \cdot \mathrm{Hz}$ & $400.40 \cdot \mathrm{cP}$ \\
\hline
\end{tabular}

\begin{tabular}{|l|c|c|c|}
\hline RHEOLOGICAL CLASSIEICATION & \multicolumn{3}{|l|}{ PSEUDOPLASTIC } \\
\hline INITIAL YIELD STRESS & $T$ & 0.0000 & Pascals \\
\hline CONSISTENCY FACTOR & $K$ & 1.4218 & Pascal-Sec \\
\hline NON-NEHTONIAN FLOW FACTOR & $n$ & 0.6767 & Dimensionless \\
\hline CURVE FIT ACCURACY & $R^{2}$ & 99.7695 & Percent(\%) \\
\hline
\end{tabular}


ENERGY \& ENVIRONMENTAL RESEARCH CENTER

COAL BENEFICIATION PROJECT

Grand Forks, North Dakota 58202

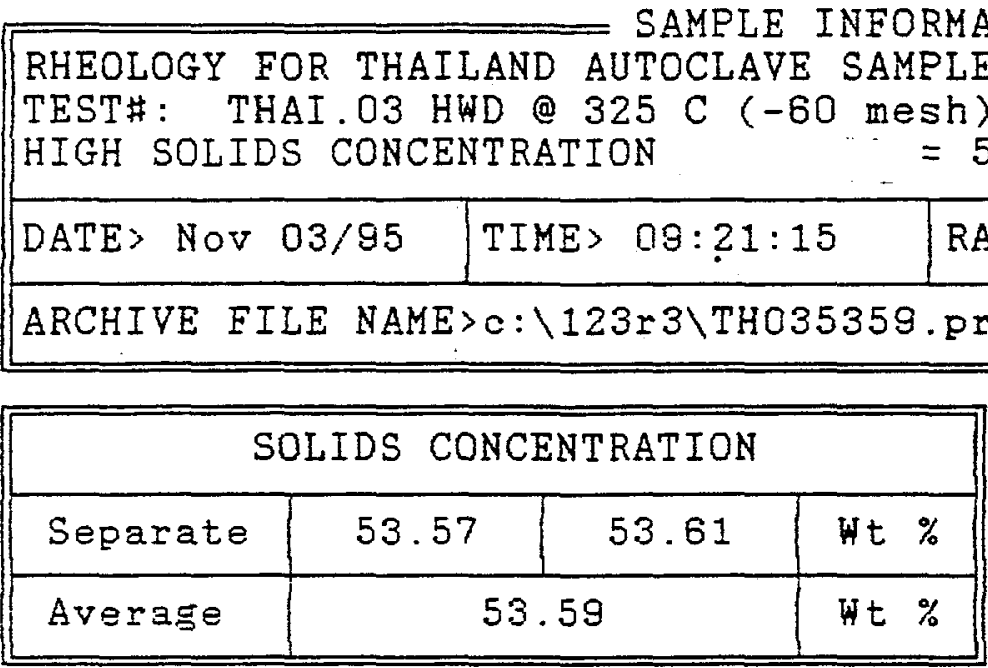

\begin{tabular}{|l|c|c|c|}
\hline \multicolumn{3}{|c|}{ ASH CONCENTRATION } \\
\hline Separate & $* * * * *$ & $* * * * *$ & Wt $\%$ \\
\hline Average & $* * * * *$ & Ht $\%$ \\
\hline
\end{tabular}

\begin{tabular}{|l|c|l|}
\hline \multicolumn{2}{|c|}{ VISCOMETER CONEIGURATION } \\
\hline Sensor & MV-IIP & Sensor Type \\
\hline Factor A & 3.76 & Pascals \\
\hline Factor M & 4.40 & $1 /$ sec \\
\hline Temperature & 25.00 & 'Celcius \\
\hline$\%$ D & 100.00 & Percent (\%) \\
\hline$\%$ Tau & 70.00 & Percent (\%) \\
\hline
\end{tabular}

\begin{tabular}{|c|c|}
\hline $\begin{array}{c}\text { SHEAR } \\
\text { RATE }\end{array}$ & $\begin{array}{l}\text { APPARENT } \\
\text { VISCOSITY }\end{array}$ \\
\hline$+50 \cdot \mathrm{Hz}$ & $829.41 \cdot \mathrm{cP}$ \\
\hline$+100 \cdot \mathrm{Hz}$ & $614.59 \cdot \mathrm{cP}$ \\
\hline$+200 \cdot \mathrm{Hz}$ & $528.40 \cdot \mathrm{cP}$ \\
\hline$+300 \cdot \mathrm{Hz}$ & $468.96 \cdot \mathrm{cP}$ \\
\hline$+400 \cdot \mathrm{Hz}$ & $411.04 \cdot \mathrm{cP}$ \\
\hline$-400 \cdot \mathrm{Hz}$ & $406.36 \cdot \mathrm{cP}$ \\
\hline$-300 \cdot \mathrm{Hz}$ & $412.83 \cdot \mathrm{cP}$ \\
\hline$-200 \cdot \mathrm{Hz}$ & $442.57 \cdot \mathrm{cP}$ \\
\hline$-100 \cdot \mathrm{Hz}$ & $529.02 \cdot \mathrm{cP}$ \\
\hline$-50 \cdot \mathrm{Hz}$ & $660.04 \cdot \mathrm{cP}$ \\
\hline
\end{tabular}

\begin{tabular}{|l|c|c|l|}
\hline RHEOLOGICAL CLASSIFICATION & \multicolumn{2}{|c|}{ PSEUDOPLASTIC } \\
\hline INITIAL YIELD STRESS & $T$ & 0.0000 & Pascals \\
\hline CONSISTENCY FACTOR & $\mathrm{K}$ & 3.2220 & ${\text { Pascal- } \text { Sec }^{n}}$ \\
\hline NON-NEHTONIAN FLOH FACTOR & $n$ & 0.6579 & Dimensionless \\
\hline CURVE FIT ACCURACY & $\mathrm{R}^{2}$ & 99.6670 & Percent(\%) \\
\hline
\end{tabular}


ENERGY \& ENVIRONMENTAL RESEARCH CENTER

COAL BENEFICIATION PROJECT

Grand Forks, North Dakota 58202

\begin{tabular}{|c|c|c|}
\hline \multicolumn{3}{|c|}{ 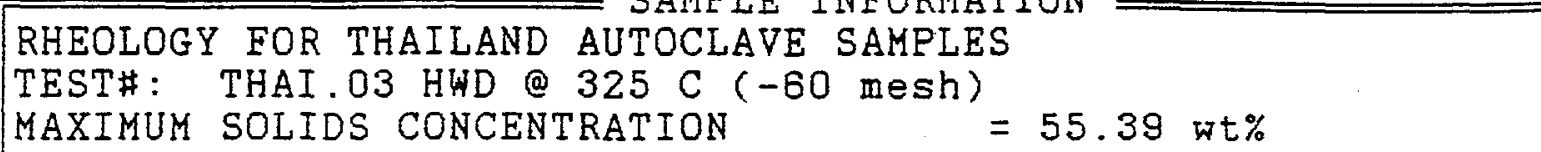 } \\
\hline DATE > NOV $03 / 95$ & TIME $>09: 10: 08$ & RATE $>120$ samples/minute \\
\hline
\end{tabular}

\begin{tabular}{|l|c|c|c|}
\hline \multicolumn{4}{|c|}{ SOI.IDE CONCENTRATION } \\
\hline Separate & 55.32 & 55.45 & Ht \% \\
\hline Average & \multicolumn{2}{|c|}{55.39} & Wt \% \\
\hline
\end{tabular}

\begin{tabular}{|l|c|c|c|}
\hline \multicolumn{3}{|c|}{ ASH CONCENTRATION } \\
\hline Separate & $* * * * *$ & $* * * * *$ & Ht $\%$ \\
\hline Average & \multirow{2}{*}{$* * * * *$} & Wt $\%$ \\
\hline
\end{tabular}

\begin{tabular}{||c|r|}
\hline $\begin{array}{c}\text { SHEAR } \\
\text { RATE }\end{array}$ & $\begin{array}{c}\text { APPARENT } \\
\text { VISCOSITY }\end{array}$ \\
\hline$+50 \cdot \mathrm{Hz}$ & $3036.95 \cdot \mathrm{cP}$ \\
\hline$+100 \cdot \mathrm{Hz}$ & $1967.47 \cdot \mathrm{cP}$ \\
\hline$+200 \cdot \mathrm{Hz}$ & $1130.88 \cdot \mathrm{cP}$ \\
\hline$+300 \cdot \mathrm{Hz}$ & $914.93 \cdot \mathrm{cP}$ \\
\hline$+400 \cdot \mathrm{Hz}$ & $759.32 \cdot \mathrm{cP}$ \\
\hline$-400 \cdot \mathrm{Hz}$ & $738.93 \cdot \mathrm{cP}$ \\
\hline$-300 \cdot \mathrm{Hz}$ & $774.95 \cdot \mathrm{cP}$ \\
\hline$-200 \cdot \mathrm{Hz}$ & $930.96 \cdot \mathrm{cP}$ \\
\hline$-100 \cdot \mathrm{Hz}$ & $1323.66 \cdot \mathrm{cP}$ \\
\hline$-50 \cdot \mathrm{Hz}$ & $1946.82 \cdot \mathrm{cP}$ \\
\hline
\end{tabular}

\begin{tabular}{|l|c|l|}
\hline \multicolumn{3}{|c|}{ VISCOMETER CONEIGURATION } \\
\hline Sensor & MV-IIP & Sensor Type \\
\hline Factor A & 3.76 & Pascals \\
\hline Factor M & 4.40 & $1 /$ sec \\
\hline Temperature & 25.00 & 'Celcius \\
\hline$\%$ D & 100.00 & Percent (\%) \\
\hline$\%$ Tau & 100.00 & Percent (\%) \\
\hline
\end{tabular}

\begin{tabular}{|c|}
\hline $\begin{array}{c}\text { SAMPIE DENSITY } \\
\left(\mathrm{g} \times \mathrm{amS} / \mathrm{cm}^{\wedge} 3\right)\end{array}$ \\
\hline$* * * * * * *$ \\
\hline
\end{tabular}

\begin{tabular}{|l|c|c|l|}
\hline RHEOLOGICAL CLASSIFICATION & \multicolumn{4}{|c|}{ YIELD POWER LAN - PSEUDOPLASTIC } \\
\hline INITIAL YIELD STRESS & $T$ & 47.3279 & Pascals \\
\hline CONSISTENCY FACTOR & $\mathbb{R}$ & 17.1418 & PasCaI•Sec \\
\hline NON-NEWTONIAN FLOW FACTOR & $n$ & 0.4511 & Dimensionless \\
\hline CURVE FIT ACCURACY & $\mathrm{R}^{2}$ & 98.3525 & Percent(\%) \\
\hline
\end{tabular}


ENERGY \& ENVIRONMENTAL RESEARCH CENTER

COAL BENEFICIATION PROJECT

Grand Forks, North Dakota 58202

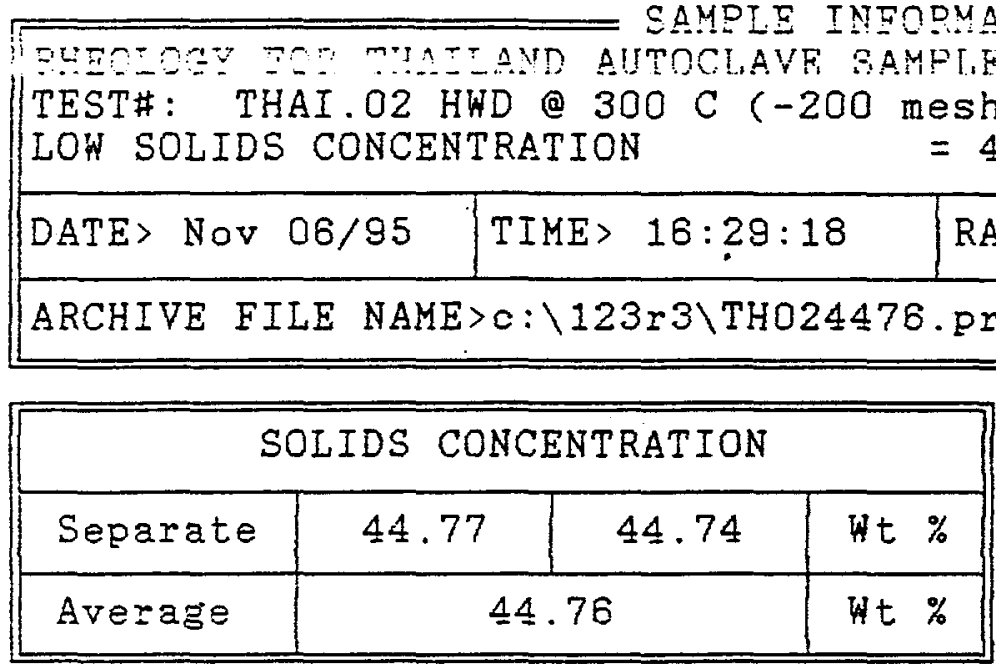

\begin{tabular}{|l|c|c|c|}
\hline \multicolumn{3}{|c|}{ ASH CONCENTRATION } \\
\hline Separate & $* * * * *$ & $* * * * *$ & Nt $\%$ \\
\hline Average & & $* * * * *$ & Nt $\%$ \\
\hline
\end{tabular}

\begin{tabular}{|l|c|l|}
\hline \multicolumn{3}{|c|}{ VISCOMETER CONEIGURATION } \\
\hline Sensor & MV-IIP & Sensor Type \\
\hline Factor A & 3.76 & Pascals \\
\hline Factor $M$ & 4.40 & $1 /$ sec \\
\hline Temperature & 25.00 & 'Celcius \\
\hline$\%$ D & 100.00 & Percent (\%) \\
\hline$\%$ Tau & 20.00 & Percent (\%) \\
\hline
\end{tabular}

\begin{tabular}{|c|c|}
\hline $\begin{array}{c}\text { SHEAR } \\
\text { RATE }\end{array}$ & $\begin{array}{c}\text { ARPARENT } \\
\text { VISCOSITY }\end{array}$ \\
\hline$+50 \cdot \mathrm{Hz}$ & $178.67 \cdot \mathrm{cP}$ \\
\hline$+100 \cdot \mathrm{Hz}$ & $124.72 \cdot \mathrm{cP}$ \\
\hline$+200 \cdot \mathrm{Hz}$ & $88.18 \cdot \mathrm{cP}$ \\
\hline$+300 \cdot \mathrm{Hz}$ & $75.24 \cdot \mathrm{cP}$ \\
\hline$+400 \cdot \mathrm{Hz}$ & $70.11 \cdot \mathrm{cP}$ \\
\hline$-400 \cdot \mathrm{Hz}$ & $71.07 \cdot \mathrm{cP}$ \\
\hline$-300 \cdot \mathrm{Hz}$ & $80.32 \cdot \mathrm{cP}$ \\
\hline$-200 \cdot \mathrm{Hz}$ & $99.34 \cdot \mathrm{cP}$ \\
\hline$-100 \cdot \mathrm{Hz}$ & $143.01 \cdot \mathrm{cP}$ \\
\hline$-50 \cdot \mathrm{Hz}$ & $226.58 \cdot \mathrm{cP}$ \\
\hline
\end{tabular}

\begin{tabular}{|l|c|c|l|}
\hline RHEOLOGICAL CLASSIEICATION & \multicolumn{3}{|c|}{ PSEUDOPLASTIC } \\
\hline INITIAL YIELD STRESS & $T$ & 0.6739 & Pascals \\
\hline CONSISTENCY FACTOR & $\mathrm{K}$ & 0.7984 & Pascal•Sec $^{n}$ \\
\hline NON-NEWTONIAN FLOW FACTOR & $n$ & 0.5837 & Dimensionless \\
\hline CURVE FIT ACCURACY & $\mathrm{R}^{2}$ & 99.5274 & Percent(\%) \\
\hline
\end{tabular}


ENERGY \& ENVIRONMENTAL RESEARCH CENTER COAL BENEFICIATION PROJECT

Grand Eorks, North Dakota 58202

SAMPLE INFORMATION
\begin{tabular}{|l|l|l||}
\hline RHEOLOGY FOR THAILAND AUTOCLAVE SAMPLES \\
TESTH: THAI.O2 HWD @ 300 C ( -200 meSh $)$ \\
MEDIUM SOLIDS CONCENTRATION & $=47.01$ wt $\%$ \\
\hline DATE $>$ NOV 06/95 & TIME $>16: 20: 21$ & RATE $>120$ samples/minute \\
\hline ARCHIVE FILE NAME >C: $\backslash 123 r 3 \backslash$ THO24701.prn \\
\hline
\end{tabular}

\begin{tabular}{|l|c|c|c|}
\hline \multicolumn{3}{|c|}{ SOLIDS CONCENTRATION } \\
\hline Separate & 47.01 & 47.00 & Wt $\%$ \\
\hline Average & \multicolumn{2}{|c|}{47.01} & Wt $\%$ \\
\hline
\end{tabular}

\begin{tabular}{|l|c|c|c|}
\hline \multicolumn{3}{|c|}{ ASH CONCENTRATION } \\
\hline Separate & $* * * * * *$ & $* * * * *$ & wt $\%$ \\
\hline Average & \multicolumn{2}{|c|}{$* * * * *$} & Ht $\%$ \\
\hline
\end{tabular}

\begin{tabular}{|c|c|}
\hline $\begin{array}{c}\text { SHEAR } \\
\text { RATE }\end{array}$ & $\begin{array}{c}\text { APPARENT } \\
\text { VISCOSITY }\end{array}$ \\
\hline$+50 \cdot \mathrm{Hz}$ & $357.13 \cdot \mathrm{cP}$ \\
\hline$+100 \cdot \mathrm{Hz}$ & $248.25 \cdot \mathrm{cP}$ \\
\hline$+200 \cdot \mathrm{Hz}$ & $172.53 \cdot \mathrm{cP}$ \\
\hline$+300 \cdot \mathrm{Hz}$ & $146.10 \cdot \mathrm{cP}$ \\
\hline$+400 \cdot \mathrm{Hz}$ & $133.85 \cdot \mathrm{cP}$ \\
\hline$-400 \cdot \mathrm{Hz}$ & $139.67 \cdot \mathrm{cP}$ \\
\hline$-300 \cdot \mathrm{Hz}$ & $160.39 \cdot \mathrm{cP}$ \\
\hline$-200 \cdot \mathrm{Hz}$ & $195.70 \cdot \mathrm{cP}$ \\
\hline$-100 \cdot \mathrm{Hz}$ & $282.93 \cdot \mathrm{cP}$ \\
\hline$-50 \cdot \mathrm{Hz}$ & $445.80 \cdot \mathrm{cP}$ \\
\hline
\end{tabular}

\begin{tabular}{|l|c|l|}
\hline \multicolumn{3}{|c|}{ VISCOMETER CONEIGURATION } \\
\hline Sensor & MV-IIP & Sensor Type \\
\hline Factor A & 3.76 & Fascals \\
\hline Factor M & 4.40 & $1 /$ sec \\
\hline Temperature & 25.00 & 'Celcius \\
\hline$\%$ D & 100.00 & Percent (\%) \\
\hline$\%$ Tau & 40.00 & Percent (\%) \\
\hline
\end{tabular}

\begin{tabular}{|c|}
\hline $\begin{array}{c}\text { SAMPLE DENSITY } \\
\left(\mathrm{grams} / \mathrm{cm}^{\wedge} 3\right)\end{array}$ \\
\hline$* * * * * * *$ \\
\hline
\end{tabular}

\begin{tabular}{|l|c|c|l|}
\hline RHEOLOGICAL CLASSIFICATION & \multicolumn{4}{|l|}{ PSEUDOPLASTIC } \\
\hline INITIAL YIEID STRESS & $T$ & 0.0000 & Pascals \\
\hline CONSISTENCY FACTOR & $\mathrm{K}$ & 1.9895 & PasCal-Sec \\
\hline NON-NENTONIAN FLOW FACTOR & $n$ & 0.5455 & Dimensionless \\
\hline CURVE FIT ACCURACY & $\mathrm{R}^{2}$ & 99.4554 & Percent(\%) \\
\hline
\end{tabular}


ENERGY \& ENVIRONMENTAL RESEARCH CENTER COAL BENEFICIATION PROJECT

Grand Forks, North Dakota 58202

\begin{tabular}{|c|c|c|c|c|}
\hline \multicolumn{5}{|c|}{$\begin{array}{l}\text { RHEOLOGY FOR THAILAND AUT } \\
\text { TEST : THAI C2 HWD O } 30 \\
\text { \#HIGH SOLIDS CONCENTRATION }\end{array}$} \\
\hline DATE $>$ NOV & \multicolumn{3}{|c|}{ TIME> $16: 10: 16$} & \\
\hline \multicolumn{5}{|c|}{ ARCHIVE FILE NAME >c: $\backslash 123 r 3 \backslash$ THO 24966 .p } \\
\hline \multicolumn{5}{|c|}{ SOLIDS CONCENTRATION } \\
\hline Separate & & 49.66 & $w t$ & \\
\hline Average & \multicolumn{2}{|c|}{49.66} & Wt & \\
\hline
\end{tabular}

\begin{tabular}{|l|c|c|c|}
\hline \multicolumn{3}{|c|}{ ASH CONCENTRATION } \\
\hline Separate & $* * * * *$ & $* * * * *$ & Ht $\%$ \\
\hline Average & ****** & Nt \% \\
\hline
\end{tabular}

\begin{tabular}{|l|c|l|}
\hline \multicolumn{3}{|c|}{ VISCOMETER CONFIGURATION } \\
\hline Sensor & MV-IIP & Sensor Type \\
\hline Factor A & 3.76 & Pascals \\
\hline Factor M & 4.40 & $1 /$ sec \\
\hline Temperature & 25.00 & ${ }^{\circ}$ Celcius \\
\hline$\%$ D & 100.00 & Percent (\%) \\
\hline$\%$ rau & 70.00 & Percent (\%) \\
\hline
\end{tabular}

\begin{tabular}{|c|c|}
\hline $\begin{array}{c}\text { SHEAR } \\
\text { RATE }\end{array}$ & $\begin{array}{l}\text { APPARENT } \\
\text { VISCOSITY }\end{array}$ \\
\hline$+50 \cdot \mathrm{Hz}$ & $1118.44 \cdot \mathrm{cP}$ \\
\hline$+100 \cdot \mathrm{Hz}$ & $783.85 \cdot \mathrm{cP}$ \\
\hline$+200 \cdot \mathrm{Hz}$ & $509.70 \cdot \mathrm{cP}$ \\
\hline$+300 \cdot \mathrm{Hz}$ & $412.68 \cdot \mathrm{cP}$ \\
\hline$+400 \cdot \mathrm{Hz}$ & $375.81 \cdot \mathrm{cP}$ \\
\hline$-400 \cdot \mathrm{Hz}$ & $389.30 \cdot \mathrm{cP}$ \\
\hline$-300 \cdot \mathrm{Hz}$ & $434.78 \cdot \mathrm{cP}$ \\
\hline$-200 \cdot \mathrm{Hz}$ & $515.21 \cdot \mathrm{cP}$ \\
\hline$-100 \cdot \mathrm{Hz}$ & $729.99 \cdot \mathrm{cP}$ \\
\hline$-50 \cdot \mathrm{Hz}$ & $1124.53 \cdot \mathrm{cP}$ \\
\hline
\end{tabular}

\begin{tabular}{|l|c|c|c|}
\hline RHEOLOGICAL CLASSIFICATION & \multicolumn{4}{|c|}{ YIELD POWER LAH - PSEUDOPLASTIC } \\
\hline INITIAL YIELD STRESS & $T$ & 2.8936 & Pascals \\
\hline CONSISTENCY FACTOR & $\mathbb{R}$ & 5.7770 & Pascal-Secn \\
\hline NON-NEHTONIAN FLON FACTOR & $n$ & 0.5394 & Dimensionless \\
\hline CURVE FIT ACCURACY & $\mathrm{R}^{2}$ & 98.6368 & Percent(\%) \\
\hline
\end{tabular}


ENERGY \& ENVIRONMENTAL RESEARCH CENTER

COAL BENEFICIATION PROJECT

Grand Forks, North Dakota 58202

\begin{tabular}{|c|c|c|}
\hline $\begin{array}{l}\text { RHEOLOGY FOR TH } \\
\text { TEST\#: THAI.02 } \\
\text { MAXIMUM SOLIDS }\end{array}$ & $\begin{array}{l}\text { LAND AUTOCLAVE } \\
\text { ID @ } 300 \mathrm{C}(-200 \\
\text { NCENTRATION }\end{array}$ & $\begin{array}{l}\text { esh) } \\
\qquad 52.08 \text { wt\% }\end{array}$ \\
\hline DATE> NOV $06 / 95$ & TIME $>15: 58: 12$ & RATE $>120$ samples/minute \\
\hline
\end{tabular}

\begin{tabular}{|l|c|c|c|}
\hline \multicolumn{4}{|c|}{ SOLIDS CONCENTRATION } \\
\hline Separate & 52.10 & 52.08 & Wt \% \\
\hline Average & \multicolumn{2}{|c|}{52.08} & Wt \% \\
\hline
\end{tabular}

\begin{tabular}{|l|c|c|c|}
\hline \multicolumn{3}{|c|}{ ASH CONCENTRATION } \\
\hline Separate & $* * * * * *$ & $* * * * *$ & wt $\%$ \\
\hline Average & \multicolumn{2}{|c|}{$* * * * *$} & wt $\%$ \\
\hline
\end{tabular}

\begin{tabular}{|c|r|}
\hline $\begin{array}{c}\text { SHEAR } \\
\text { RATE }\end{array}$ & $\begin{array}{c}\text { APPARENT } \\
\text { VISCOSITY }\end{array}$ \\
\hline$+50 \cdot \mathrm{Hz}$ & $3771.94 \cdot \mathrm{cP}$ \\
\hline$+100 \cdot \mathrm{Hz}$ & $2209.50 \cdot \mathrm{cP}$ \\
\hline$+200 \cdot \mathrm{Hz}$ & $1392.91 \cdot \mathrm{cP}$ \\
\hline$+300 \cdot \mathrm{Hz}$ & $1088.34 \cdot \mathrm{cP}$ \\
\hline$+400 \cdot \mathrm{Hz}$ & $904.07 \cdot \mathrm{cP}$ \\
\hline$-400 \cdot \mathrm{Hz}$ & $871.91 \cdot \mathrm{cP}$ \\
\hline$-300 \cdot \mathrm{Hz}$ & $908.09 \cdot \mathrm{cP}$ \\
\hline$-200 \cdot \mathrm{Hz}$ & $1025.45 \cdot \mathrm{cP}$ \\
\hline$-100 \cdot \mathrm{Hz}$ & $1416.84 \cdot \mathrm{cP}$ \\
\hline$-50 \cdot \mathrm{Hz}$ & $2108.43 \cdot \mathrm{cP}$ \\
\hline
\end{tabular}

\begin{tabular}{|l|c|l|}
\hline \multicolumn{2}{|c|}{ VISCOMETER CONEIGURATION } \\
\hline Sensor & MV-IIP & Sensor Type \\
\hline Factor A & 3.76 & Pascals \\
\hline Eactor M & 4.40 & $1 /$ sec \\
\hline Temperature & 25.00 & ${ }^{\circ}$ Celcius \\
\hline$\%$ D & 100.00 & Percent (\%) \\
\hline$\%$ Tau & 100.00 & Percent (\%) \\
\hline
\end{tabular}

\begin{tabular}{|c|}
\hline $\begin{array}{c}\text { SAMPLE DENSITY } \\
\left(\mathrm{grams} / \mathrm{cm}^{\wedge} 3\right)\end{array}$ \\
\hline$* * * * * *$ \\
\hline
\end{tabular}

\begin{tabular}{|l|c|c|c|l|}
\hline RHEOLOGICAL CLASSIFICATION & \multicolumn{4}{|c|}{ YIELD PONER LAH - PSEUDOPLASTIC } \\
\hline INITIAL YIELD STRESS & $\tau$ & 45.4242 & PasCals \\
\hline CONSISTENCY FACTOR & $\mathrm{R}$ & 20.9405 & PasCal-Sec $^{n}$ \\
\hline NON-NEWTONIAN FLOW FACTOR & $n$ & 0.4549 & Dimensionless \\
\hline CURVE FIT ACCURACY & $R^{2}$ & 96.6830 & Percent(\%) \\
\hline
\end{tabular}


ENERGY \& ENYIRONMENTAL RESEARCH CENTER

COAL BENEEICIATION PROJECT

Grand Forks, North Dakota 58202

SAMPLE INFORMATION

RHEOLOGY FOR THAILAND AUTOCLAVE SAMPLES

TEST\#: THAI.04 HWD @ $275 \mathrm{C}(-60$ mesh $)$

MAXIMUM SOLIDS CONCENTRATION $=47.09$ th

\begin{tabular}{|c|c|c|}
\hline DATE> Nov $03 / 95$ & TIME $>15: 57: 26$ & RATE: 120 same? \\
\hline
\end{tabular}

\begin{tabular}{|c|c|c|c|}
\hline \multicolumn{4}{|c|}{ SOLIDS CONCENTRATION } \\
\hline Separate & 46.96 & 47.22 & Wt \% \\
\hline Average & \multicolumn{2}{|c|}{47.09} & Wt \% \\
\hline
\end{tabular}

ASH CONCENTRATION

\begin{tabular}{|l|c|c|c|}
\hline Separate & $* * * * * * *$ & $* * * * *$ & Wt \% \\
\hline Average & ******* & Wt \% \\
\hline
\end{tabular}

\begin{tabular}{|l|c|l|}
\hline \multicolumn{2}{|c|}{ VISCOMETER CONFIGURATION } \\
\hline Sensor & MV-IIP & Sensor Type \\
\hline Eactor A & 3.76 & Pascals \\
\hline Fagtor M & 4.40 & $1 / 300$ \\
\hline Temperature & 25.00 & ${ }^{\circ}$ Celcius \\
\hline$\%$ D & 100.00 & Percent (\%) \\
\hline$\%$ Tau & 100.00 & Percent (\%) \\
\hline
\end{tabular}

\begin{tabular}{|c|c|}
\hline $\begin{array}{c}\text { SHEAR } \\
\text { RATE }\end{array}$ & $\begin{array}{l}\text { APPARENT } \\
\text { VISCOSITY }\end{array}$ \\
\hline$+50 \cdot \mathrm{Hz}$ & $2101.64 \cdot \mathrm{cP}$ \\
\hline$+100 \cdot \mathrm{Hz}$ & $1293.21 \cdot \mathrm{cP}$ \\
\hline$+200 \cdot \mathrm{Hz}$ & $824.23 \cdot \mathrm{cP}$ \\
\hline$+300 \cdot \mathrm{Hz}$ & $632.29 \cdot \mathrm{cP}$ \\
\hline$+400 \cdot \mathrm{Hz}$ & $490.27 \cdot \mathrm{cP}$ \\
\hline$-400 \cdot \mathrm{Hz}$ & $451.37 \cdot \mathrm{cP}$ \\
\hline$-300 \cdot \mathrm{Hz}$ & $419.24 \cdot \mathrm{cP}$ \\
\hline$-200 \cdot \mathrm{Hz}$ & $436.41 \cdot \mathrm{cP}$ \\
\hline$-100 \cdot \mathrm{Hz}$ & $527.95 \cdot \mathrm{cP}$ \\
\hline$-50 \cdot \mathrm{Hz}$ & $715.95 \cdot \mathrm{cP}$ \\
\hline
\end{tabular}

\begin{tabular}{|l|c|c|l|}
\hline RHEOLOGICAL CLASSIFICATION & \multicolumn{4}{|c|}{ YIELD POWER LAW - PSEUDOPLASTIC } \\
\hline INITIAL YIELD STRESS & $T$ & 35.6043 & PasCals \\
\hline CONSISTENCY FACTOR & $\mathrm{K}$ & 11.4133 & PasCal-Sec \\
\hline NON-NEWTONIAN FLOW FACTOR & $n$ & 0.4517 & DimensionlesS \\
\hline CURVE FIT ACCURACY & $\mathrm{R}^{2}$ & 98.1318 & Percent(\%) \\
\hline
\end{tabular}


ENERGY \& ENVIRONMENTAL RESEARCH CENTER

COAL BENEFICIATION PROJECT

Grand Forks, North Dakota 58202

\begin{tabular}{|c|c|c|c|c|}
\hline \multicolumn{5}{|c|}{$\begin{array}{l}\text { RHEOLOGY FOR THAILAND AUTOCLAVE SAMPI } \\
\text { TEST\#: THAI.O4 HWD @ } 275 \text { C }(-60 \text { mesh } \\
\text { HIGH SOLIDS CONCENTRATION }=46.26 \text { wt } \%\end{array}$} \\
\hline DATE $>\mathrm{NOV}$ & \multicolumn{4}{|c|}{ TIME > 16:09:38 } \\
\hline \multicolumn{5}{|c|}{ ARCHIVE FILE NAME >C: $\backslash 123 r 3 \backslash$ TH044626. } \\
\hline \multicolumn{5}{|c|}{ SOLIDS CONCENTRATION } \\
\hline Separate & & 46.22 & & \\
\hline Average & & & & \\
\hline
\end{tabular}

\begin{tabular}{|l|c|c|c|}
\hline \multicolumn{3}{|c|}{ ASH CONCENTRATION } \\
\hline Separate & $* * * * * *$ & $* * * * *$ & Wt \% \\
\hline Average & ****** & Ht \% \\
\hline
\end{tabular}

\begin{tabular}{|l|c|l|}
\hline \multicolumn{3}{|c|}{ VISCOMETER CONFIGURATION } \\
\hline Sensor & MV-IIP & Sensor Type \\
\hline Eactor A & 3.75 & Pascals \\
\hline Factor M & 4.40 & $1 /$ sec \\
\hline Temperature & 25.00 & OCeleius \\
\hline$\%$ D & 100.00 & Percent (\%) \\
\hline$\%$ Tau & 70.00 & Percent (\%) \\
\hline
\end{tabular}

\begin{tabular}{|c|c|}
\hline $\begin{array}{c}\text { SHEAR } \\
\text { RATE }\end{array}$ & $\begin{array}{l}\text { APPARENT } \\
\text { VISCOSITY }\end{array}$ \\
\hline$+50 \cdot \mathrm{Hz}$ & $1166.78 \cdot \mathrm{cP}$ \\
\hline$+100 \cdot \mathrm{Hz}$ & $820.68 \cdot \mathrm{cP}$ \\
\hline$+200 \cdot \mathrm{Hz}$ & $548.93 \cdot \mathrm{cP}$ \\
\hline$+300 \cdot \mathrm{Hz}$ & $458.74 \cdot \mathrm{cP}$ \\
\hline$+400 \cdot \mathrm{Hz}$ & $392.11 \cdot \mathrm{cP}$ \\
\hline$-400 \cdot \mathrm{Hz}$ & $365.97 \cdot \mathrm{cP}$ \\
\hline$-300 \cdot \mathrm{Hz}$ & $351.25 \cdot \mathrm{cP}$ \\
\hline$-200 \cdot \mathrm{Hz}$ & $368.46 \cdot \mathrm{cP}$ \\
\hline$-100 \cdot \mathrm{Hz}$ & $457.45 \cdot \mathrm{cP}$ \\
\hline$-50 \cdot \mathrm{Hz}$ & $622.68 \cdot \mathrm{cP}$ \\
\hline
\end{tabular}

\begin{tabular}{|l|c|c|c|}
\hline RHEOLOGICAL CLASSIFICATION & \multicolumn{3}{|c|}{ YIELD POWER LAH - PSEUDOPLASTIC } \\
\hline INITIAL YIELD STRESS & $T$ & 12.7876 & Pascals \\
\hline CONSISTENCY FACTOR & $\mathrm{K}$ & 4.6338 & Pascal-Sec $^{n}$ \\
\hline NON-NEWTONIAN FLOW FACTOR & $n$ & 0.5754 & Dimensionless \\
\hline CURVE FIT ACCURACY & $\mathrm{R}^{2}$ & 98.9794 & Percent(\%) \\
\hline
\end{tabular}


ENERGY \& ENVIRONMENTAL RESEARCH CENTER COAL BENEFICIATION PROJECT

Grand Forks, North Dakota 58202

SAMPLE INFORMATION

RHEOLOGY FOR THAILAND AUTOCLAVE SAMPLES

TEST\#: THAI.04 HHD @ $275 \mathrm{C}$ ( -60 mesh)

MEDIUM SOLIDS CONCENTRATION $=45.03$ wt $\%$

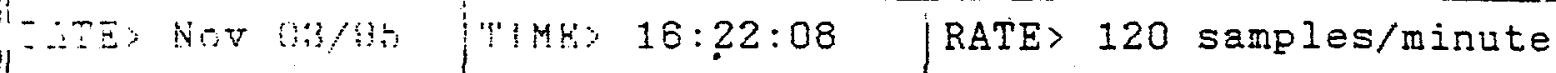

ARCHIVE FILE NAME >c: \123r3\TH044503.prn

\begin{tabular}{|c|c|c|c|}
\hline \multicolumn{4}{|c|}{ SOLIDS CONCENTRATION } \\
\hline Separate & 45.02 & 45.03 & Wt \% \\
\hline Average & \multicolumn{2}{|c|}{45.03} & Wt $\%$ \\
\hline
\end{tabular}

\begin{tabular}{|l|c|c|c|}
\hline \multicolumn{4}{|c|}{ ASH CONCENTRATION } \\
\hline Separate & $* * * * * *$ & $* * * * *$ & Wt $\%$ \\
\hline Average & \multicolumn{2}{|c|}{$* * * * *$} & Ht $\%$ \\
\hline
\end{tabular}

\begin{tabular}{|l|c|l|}
\hline \multicolumn{2}{|c|}{ VISCOMETER CONEIGURATION } \\
\hline Sensor & MV-IIP & Sensor Type \\
\hline Factor A & 3.76 & Pascals \\
\hline Factor M & 4.40 & $1 /$ sec \\
\hline Temperature & 25.00 & 'Celcius \\
\hline$\%$ D & 100.00 & Percent (\%) \\
\hline$\%$ Tau & 40.00 & Percent (\%) \\
\hline
\end{tabular}

\begin{tabular}{|c|c|}
\hline $\begin{array}{c}\text { SHEAR } \\
\text { RATE }\end{array}$ & $\begin{array}{c}\text { APPARENT } \\
\text { VISCOSITY }\end{array}$ \\
\hline$+50 \cdot \mathrm{Hz}$ & $523.60 \cdot \mathrm{cP}$ \\
\hline$+100 \cdot \mathrm{Hz}$ & $393.09 \cdot \mathrm{cP}$ \\
\hline$+200 \cdot \mathrm{Hz}$ & $308.19 \cdot \mathrm{cP}$ \\
\hline$+300 \cdot \mathrm{Hz}$ & $279.22 \cdot \mathrm{cP}$ \\
\hline$+400 \cdot \mathrm{Hz}$ & $250.88 \cdot \mathrm{cP}$ \\
\hline$-400 \cdot \mathrm{Hz}$ & $246.59 \cdot \mathrm{cP}$ \\
\hline$-300 \cdot \mathrm{Hz}$ & $240.06 \cdot \mathrm{cP}$ \\
\hline$-200 \cdot \mathrm{Hz}$ & $252.19 \cdot \mathrm{cP}$ \\
\hline$-100 \cdot \mathrm{Hz}$ & $302.02 \cdot \mathrm{cP}$ \\
\hline$-50 \cdot \mathrm{Hz}$ & $404.36 \cdot \mathrm{cP}$ \\
\hline
\end{tabular}

\begin{tabular}{|l|c|c|l|}
\hline RHEOLOGICAL CLASSIFICATION & \multicolumn{4}{|c|}{ PSEUDOPLASTIC } \\
\hline INITIAL YIELD STRESS & $\tau$ & 0.1129 & Pascals \\
\hline CONSISTENCY FACTOR & $K$ & 1.6375 & Pascal-Sec $^{n}$ \\
\hline NON-NEWTONIAN FLOW FACTOR & $n$ & 0.6881 & Dimensionless \\
\hline CURVE FIT ACCURACY & $\mathrm{R}^{2}$ & 99.7285 & Percent(\%) \\
\hline
\end{tabular}


ENERGY \& ENVIRONMENTAL RESEARCH CENTER

COAL BENEFICIATION PROJECT

Grand Forks, North Dakota 58202

SAMPLE INFORMATION

RHEOLOGY FOR THAILAND AUTOCLAVE SAMPLES

TEST\#: THAI.04 HWD @ $275 \mathrm{C}$ (-60 mesh)

LOW SOLIDS CONCENTRATION a 43.51 wt\%

\begin{tabular}{|l|l|l|l|l|l|l|}
\hline DATE $>$ Nov 03/95 & TIME $16: 30: 44$ & RATE $>120$ samples/minute
\end{tabular}

ARCHIVE FILE NAME>c: \123r3\TH044351.prn

\begin{tabular}{|l|c|c|c|}
\hline \multicolumn{4}{|c|}{ SOLIDS CONCENTRATION } \\
\hline Separate & 43.49 & 43.53 & Wt \% \\
\hline Average & \multicolumn{2}{|c|}{43.51} & Wt \% \\
\hline
\end{tabular}

\begin{tabular}{|l|c|c|c|}
\hline \multicolumn{3}{|c|}{ ASH CONCENTRATION } \\
\hline Separate & $* * * * *$ & $* * * * *$ & wt $\%$ \\
\hline Average & \multicolumn{2}{|c|}{$* * * * *$} & wt $\%$ \\
\hline
\end{tabular}

\begin{tabular}{|l|c|l|}
\hline \multicolumn{3}{|c|}{ VISCOMETER CONFIGURATION } \\
\hline Sersor & MV-IIP & Sensor Type \\
\hline Factor A & 3.76 & Pascals \\
\hline Factor M & 4.40 & $1 /$ sec \\
\hline Temperature & 25.00 & 'Celcius \\
\hline$\%$ D & 100.00 & Percent (\%) \\
\hline$\%$ Tau & 20.00 & Percent (\%) \\
\hline
\end{tabular}

\begin{tabular}{|c|c|}
\hline $\begin{array}{c}\text { SHEAR } \\
\text { RATE }\end{array}$ & $\begin{array}{c}\text { APPARENT } \\
\text { VISCOSITY }\end{array}$ \\
\hline$+50 \cdot \mathrm{Hz}$ & $295.43 \cdot \mathrm{cP}$ \\
\hline$+100 \cdot \mathrm{Hz}$ & $231.58 \cdot \mathrm{cP}$ \\
\hline$+200 \cdot \mathrm{Hz}$ & $184.97 \cdot \mathrm{cP}$ \\
\hline$+300 \cdot \mathrm{Hz}$ & $170.89 \cdot \mathrm{cP}$ \\
\hline$+400 \cdot \mathrm{Hz}$ & $159.07 \cdot \mathrm{cP}$ \\
\hline$-400 \cdot \mathrm{Hz}$ & $159.85 \cdot \mathrm{cP}$ \\
\hline$-300 \cdot \mathrm{Hz}$ & $159.51 \cdot \mathrm{cP}$ \\
\hline$-200 \cdot \mathrm{Hz}$ & $170.52 \cdot \mathrm{cP}$ \\
\hline$-100 \cdot \mathrm{Hz}$ & $209.97 \cdot \mathrm{cP}$ \\
\hline$-50 \cdot \mathrm{Hz}$ & $291.75 \cdot \mathrm{cP}$ \\
\hline
\end{tabular}

\begin{tabular}{|l|c|c|l|}
\hline RHEOLOGICAL CLASSIFICATION & \multicolumn{4}{|c|}{ PSEUDOPLASTIC } \\
\hline INITIAL YIELD STRESS & $T$ & 0.0000 & Pascals \\
\hline CONSISTENCY FACTOR & $\mathbb{R}$ & 0.9309 & Pascal-Sec $^{n}$ \\
\hline NON-NEWTONIAN FLOW FACTOR & $n$ & 0.7021 & Dimensionless \\
\hline CURVE FIT ACCURACY & $R^{2}$ & 99.8432 & Percent(\%) \\
\hline
\end{tabular}


ENERGY \& ENVIRONMENTAL RESEARCH CENTER

COAL BENEFICIATION PROJECT

Grand Forks, North Dakota 58202

SAMPLE INFORMATION

RHEOLOGY FOR THAILAND AUTOCLAVE SAMPLES

TEST\#: THAI.03 HWD @ $325 \mathrm{C}$ ( -60 mesh)

MAXIMUM SOLIDS CONCENTRATION $=51.89 \mathrm{wt} \%$

\begin{tabular}{|l|l|l|l|l|l|}
\hline DATE $>$ Nov 02/95 & TIME> 11:18:30 & RATE> 120 samples/minute
\end{tabular}

ARCHIVE FILE NAME>c: \123r3\TH035189.prn

\begin{tabular}{|c|c|c|c|}
\hline \multicolumn{4}{|c|}{ SOLIDS CONCENTRATION } \\
\hline Separate & 51.87 & 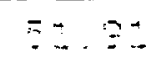 & $y \pm \%$ \\
\hline Average & & & Wt \% \\
\hline
\end{tabular}

\begin{tabular}{|l|c|c|c|}
\hline \multicolumn{3}{|c|}{ ASH CONCENTRATION } \\
\hline Separate & $* * * * *$ & $* * * * *$ & Ht $\%$ \\
\hline Average & \multicolumn{2}{|c|}{$* * * * *$} & Ht $\%$ \\
\hline
\end{tabular}

\begin{tabular}{|l|c|l|}
\hline \multicolumn{2}{|c|}{ VISCOMETER CONFIGURATION } \\
\hline Sensor & MV-IIP & Sensor Type \\
\hline Factor A & 3.76 & Pascals \\
\hline Factor M & 4.40 & $1 /$ sec \\
\hline Temperature & 25.00 & ${ }^{\circ}$ Celcius \\
\hline$\%$ D & 100.00 & Percent (\%) \\
\hline$\%$ Tau & 100.00 & Percent (\%) \\
\hline
\end{tabular}

\begin{tabular}{|c|c|}
\hline $\begin{array}{c}\text { SHEAR } \\
\text { RATE }\end{array}$ & $\begin{array}{c}\text { APPARENT } \\
\text { VISCOSITY }\end{array}$ \\
\hline$+50 \cdot \mathrm{Hz}$ & $2066.77 \cdot \mathrm{cP}$ \\
\hline$+100 \cdot \mathrm{Hz}$ & $1204.90 \cdot \mathrm{cP}$ \\
\hline$+200 \cdot \mathrm{Hz}$ & $664.20 \cdot \mathrm{cP}$ \\
\hline$+300 \cdot \mathrm{Hz}$ & $503.25 \cdot \mathrm{cP}$ \\
\hline$+400 \cdot \mathrm{Hz}$ & $444.69 \cdot \mathrm{cP}$ \\
\hline$-400 \cdot \mathrm{Hz}$ & $444.30 \cdot \mathrm{cP}$ \\
\hline$-300 \cdot \mathrm{Hz}$ & $440.94 \cdot \mathrm{cP}$ \\
\hline$-200 \cdot \mathrm{Hz}$ & $469.53 \cdot \mathrm{cP}$ \\
\hline$-100 \cdot \mathrm{Hz}$ & $595.30 \cdot \mathrm{cP}$ \\
\hline$-50 \cdot \mathrm{Hz}$ & $828.96 \cdot \mathrm{cP}$ \\
\hline
\end{tabular}

\begin{tabular}{|c|c|c|c|}
\hline RHEOLOGICAL CLASSIFICATION & YIELD & ER LAW - & EUDOPLASTIC \\
\hline INITIAL YIELD STRESS & $\tau$ & 18.6870 & Pascals \\
\hline COHSIDTENOZ FAOTOF & T & 17.5587 & 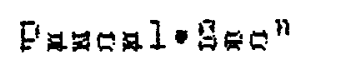 \\
\hline NON-NEWTONIAN FLOW FACTOR & n & 0.3635 & Dimensionless \\
\hline CURVE FIT ACCURACY & $R^{2}$ & 94.7160 & Percent $(\%)$ \\
\hline
\end{tabular}


ENERGY \& ENVIRONMENTAL RESEARCH CENTER

COAL BENEFICIATION PROJECT

Grand Forks, North Dakota 58202

- SAMPLE INFORMATION

LOGY FOR THAILAND AUTOCLAVE SAMPLES

TEST\#: THAI.03 HWD @ $325 \mathrm{C}$ (-60 mesh)

HIGH SOLIDS CONCENTRATION $=50.23 \mathrm{wt} \%$

\begin{tabular}{|l|l|l|l|l|l|l|}
\hline DATE $>$ Nov 02/95 & TIME> 11:33:11 & RATE> 120 samples/minute
\end{tabular}

ARCHIVE FILE NAME>C: \123r3\THO35023.prn

\begin{tabular}{|l|c|c|c|}
\hline \multicolumn{3}{|c|}{ SOLIDS CONCENTRATION } \\
\hline Separate & 50.26 & 50.20 & Wt \% \\
\hline Average & 50.23 & Wt \% \\
\hline
\end{tabular}

\begin{tabular}{|c|c|c|c|}
\hline \multicolumn{4}{|c|}{ ASH CONCENTRATION } \\
\hline Separate & $* * * * * *$ & $* * * * * *$ & Wt $\%$ \\
\hline Average & \multicolumn{2}{|c|}{$* * * * *$} & Wt \% \\
\hline
\end{tabular}

\begin{tabular}{|l|c|l|}
\hline \multicolumn{3}{|c|}{ VISCOMETER CONEIGURATION } \\
\hline Sensor & MV-IIP & Sensor Type \\
\hline Eactor A & 3.76 & Pascals \\
\hline Factor M & 4.40 & $1 /$ sec \\
\hline Temperature & 25.00 & 'Celeius \\
\hline$\%$ D & 100.00 & Percent (\%) \\
\hline$\%$ Tau & 70.00 & Percent (\%) \\
\hline
\end{tabular}

\begin{tabular}{|c|c|}
\hline $\begin{array}{c}\text { SHEAR } \\
\text { RATE }\end{array}$ & $\begin{array}{l}\text { APPARENT } \\
\text { VISCOSITY }\end{array}$ \\
\hline$+50 \cdot \mathrm{Hz}$ & $1243.91 \cdot \mathrm{cP}$ \\
\hline$+100 \cdot \mathrm{Hz}$ & $652.24 \cdot \mathrm{cP}$ \\
\hline$+200 \cdot \mathrm{Hz}$ & $349.47 \cdot \mathrm{cP}$ \\
\hline$+300 \cdot \mathrm{Hz}$ & $270.27 \cdot \mathrm{cP}$ \\
\hline$+400 \cdot \mathrm{Hz}$ & $249.40 \cdot \mathrm{cP}$ \\
\hline$-400 \cdot \mathrm{Hz}$ & $241.56 \cdot \mathrm{cP}$ \\
\hline$-300 \cdot \mathrm{Hz}$ & $259.64 \cdot \mathrm{cP}$ \\
\hline$-200 \cdot \mathrm{Hz}$ & $280.55 \cdot \mathrm{cP}$ \\
\hline$-100 \cdot \mathrm{Hz}$ & $346.95 \cdot \mathrm{cP}$ \\
\hline$-50 \cdot \mathrm{Hz}$ & $471.35 \cdot \mathrm{cP}$ \\
\hline
\end{tabular}

\begin{tabular}{|l|c|c|c|}
\hline BHEOLOGICAL CLASSIFICATION & \multicolumn{4}{|c|}{ YIELD POWER LAW - PSEUDOPLASTIC } \\
\hline INITIAL YIELD STRESS & $T$ & 14.9233 & Pascals \\
\hline CONSISTENCY FACTOR & $R$ & 9.9307 & Pascal-Sec $^{n}$ \\
\hline NON-NEWTONIAN FLOW FACTOR & $n$ & 0.3412 & Dimensionless \\
\hline CURVE FIT ACCURACY & $R^{2}$ & 92.4495 & Percent $(\%)$ \\
\hline
\end{tabular}


ENERGY \& ENVIRONMENTAL RESEARCH CENTER

COAL BENEFICIATION PROJECT

Grand Forks, North Dakota 58202

SAMPLE INFORMATION
\begin{tabular}{|l|l|l||}
\hline RHEOLOGY FOR THAILAND AUTOCLAVE SAMPLES \\
TEST\#: THAI.O3 HWD @ 325 C $(-60$ mesh) \\
MEDIUM SOLIDS CONCENTRATION $=48.66$ Wt $\%$ \\
\hline DATE $>$ NOV 02/95 & TIME $>11: 47: 33$ & RATE $>120$ samples/minute \\
\hline ARCHIVE FILE NAME >C: $\backslash 123$ r $3 \backslash$ THO34866.prn \\
\hline
\end{tabular}

\begin{tabular}{|l|c|c|c|}
\hline \multicolumn{3}{|c|}{ SOLIDS CONCENTRATION } \\
\hline Separate & 48.70 & 48.62 & $\mathrm{t} \%$ \\
\hline Average & \multicolumn{4}{|c|}{48.66} & Wt $\%$ \\
\hline
\end{tabular}

\begin{tabular}{|l|c|c|c|}
\hline \multicolumn{3}{|c|}{ ASH CONCENTRATION } \\
\hline Separate & $* * * * *$ & $* * * * *$ & Wt \% \\
\hline Average & \multicolumn{2}{|c|}{$* * * * *$} & Wt \% \\
\hline
\end{tabular}

\begin{tabular}{|l|c|l|}
\hline \multicolumn{2}{|c|}{ VISCOMETER CONFIGURATION } \\
\hline Sensor & MV-IIP & Sensor Type \\
\hline Factor A & 3.76 & Pascals \\
\hline Factor M & 4.40 & 1 / sec \\
\hline Temperature & 25.00 & 'Celcius \\
\hline$\%$ D & 100.00 & Percent (\%) \\
\hline$\%$ Tau & 40.00 & Percent (\%) \\
\hline
\end{tabular}

\begin{tabular}{|c|c|}
\hline $\begin{array}{c}\text { SUEAE } \\
\text { RATE }\end{array}$ & $\begin{array}{l}\text { APEAEES } \\
\text { VISCOSITY }\end{array}$ \\
\hline$+50 \cdot \mathrm{Hz}$ & $668.53 \cdot \mathrm{cP}$ \\
\hline$+100 \cdot \mathrm{Hz}$ & $479.21 \cdot \mathrm{cP}$ \\
\hline$+200 \cdot \mathrm{Hz}$ & $320.71 \cdot \mathrm{cP}$ \\
\hline$+300 \cdot \mathrm{Hz}$ & $274.62 \cdot \mathrm{cP}$ \\
\hline$+400 \cdot \mathrm{Hz}$ & $242.88 \cdot \mathrm{cP}$ \\
\hline$-400 \cdot \mathrm{Hz}$ & $233.38 \cdot \mathrm{cP}$ \\
\hline$-300 \cdot \mathrm{Hz}$ & $234.02 \cdot \mathrm{cP}$ \\
\hline$-200 \cdot \mathrm{Hz}$ & $256.21 \cdot \mathrm{cP}$ \\
\hline$-100 \cdot \mathrm{Hz}$ & $326.78 \cdot \mathrm{cP}$ \\
\hline$-50 \cdot \mathrm{Hz}$ & $474.09 \cdot \mathrm{cP}$ \\
\hline
\end{tabular}

\begin{tabular}{|l|c|c|l|}
\hline RHEOLOGICAL CLASSIFICATION & \multicolumn{4}{|c|}{ YIELD POHER LAL - PSEUDOFLASTIC } \\
\hline INITIAL YIELD STRESS & $\mathrm{T}$ & 1.1274 & Pascals \\
\hline CONSISTENCY FACTOR & $\mathrm{K}$ & 3.0148 & Pascal-Sec \\
\hline NON-NEWTONIAN FLOW FACTOR & $n$ & 0.5791 & Dimensionless \\
\hline CURVE FIT ACCURACY & $\mathrm{R}^{2}$ & 99.0889 & Percent(\%) \\
\hline
\end{tabular}


ENERGY \& ENVIRONMENTAL RESEARCH CENTER

COAL BENEFICIATION PROJECT

Grand Forks, North Dakota 58202

SAMPLE INFORMATION
\begin{tabular}{|l|l|l||}
\hline RHEOLOGY FOR THAILAND AUTOCLAVE SAMPLES \\
TEST : THAI.03 HWD @ $325 \mathrm{C}(-60 \mathrm{mesh})$ \\
LOW SOLIDS CONCENTRATION $=47.13 \mathrm{wt \%}$ \\
\hline DATE> NOV 02/95 & TIME $>11: 58: 40$ & RATE $>120$ samples/minute \\
\hline ARCHIVE FILE NAME>C: $\backslash 123 \times 3 \backslash$ THO34713.prn \\
\hline
\end{tabular}

\begin{tabular}{|c|c|c|c|}
\hline \multicolumn{4}{|c|}{ SOLIDS CONCENTRATION } \\
\hline Separate & 47.12 & 47.14 & wt \% \\
\hline Average & \multicolumn{2}{|c|}{47.13} & Ht \% \\
\hline
\end{tabular}

\begin{tabular}{|l|c|c|c|}
\hline \multicolumn{3}{|c|}{ ASH CONCENTRATION } \\
\hline Separate & $* * * * * * *$ & $* * * * *$ & wt $\%$ \\
\hline Average & \multicolumn{2}{|c|}{$* * * * *$} & wt $\%$ \\
\hline
\end{tabular}

\begin{tabular}{|l|c|l|}
\hline \multicolumn{2}{|c|}{ VISCOMETER CONFIGURATION } \\
\hline Sensor & MV-IIP & Sensor Type \\
\hline Factor A & 3.76 & Pascals \\
\hline Factor M & 4.40 & $1 /$ sec \\
\hline Temperature & 25.00 & ${ }^{\circ}$ Celcius \\
\hline$\%$ D & 100.00 & Percent (\%) \\
\hline$\%$ Tau & 20.00 & Percent (\%) \\
\hline
\end{tabular}

\begin{tabular}{|c|c|}
\hline $\begin{array}{c}\text { SHEAR } \\
\text { RATE }\end{array}$ & $\begin{array}{c}\text { APPARENT } \\
\text { VISCOSITY }\end{array}$ \\
\hline$+50 \cdot \mathrm{Hz}$ & $266.68 \cdot \mathrm{cP}$ \\
\hline$+100 \cdot \mathrm{Hz}$ & $205.49 \cdot \mathrm{cP}$ \\
\hline$+200 \cdot \mathrm{Hz}$ & $163.67 \cdot \mathrm{cP}$ \\
\hline$+300 \cdot \mathrm{Hz}$ & $150.84 \cdot \mathrm{cP}$ \\
\hline$+400 \cdot \mathrm{Hz}$ & $143.03 \cdot \mathrm{cP}$ \\
\hline$-400 \cdot \mathrm{Hz}$ & $147.06 \cdot \mathrm{cP}$ \\
\hline$-300 \cdot \mathrm{Hz}$ & $152.23 \cdot \mathrm{cP}$ \\
\hline$-200 \cdot \mathrm{Hz}$ & $163.45 \cdot \mathrm{cP}$ \\
\hline$-100 \cdot \mathrm{Hz}$ & $203.33 \cdot \mathrm{cP}$ \\
\hline$-50 \cdot \mathrm{Hz}$ & $282.08 \cdot \mathrm{cP}$ \\
\hline
\end{tabular}

\begin{tabular}{|l|c|c|l|}
\hline RHEOLOGICAL CLASSIFICATION & \multicolumn{4}{|c|}{ PSEUDOPLASTIC } \\
\hline INITIAL YIELD STRESS & $\tau$ & 0.2707 & Pascals \\
\hline CONSISTENCY FACTOR & $K$ & 0.8032 & Pascal•Sec $^{n}$ \\
\hline NON-NEWTONIAN FLOW FACTOR & $n$ & 0.7056 & Dimensionless \\
\hline CURVE FIT ACCURACY & $\mathrm{R}^{2}$ & 99.8192 & Percent $(\%)$ \\
\hline
\end{tabular}


ENERGY \& ENVIRONMENTAL RESEARCH CENTER

COAL BENEFICIATION PROJECT

Grand Forks, North Dakota 58202

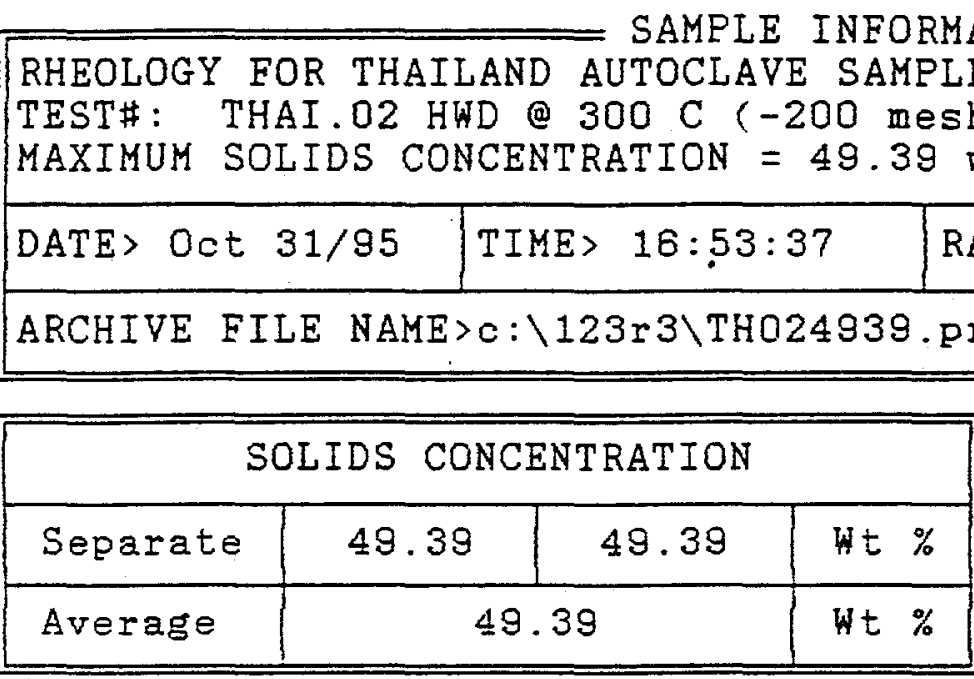

\begin{tabular}{|l|c|c|c|}
\hline \multicolumn{3}{|c|}{ ASE CONCEATEATTOU } \\
\hline Separate & $* * * * *$ & $* * * * *$ & Wt \% \\
\hline Average & $* * * * *$ & Wt \% \\
\hline
\end{tabular}

\begin{tabular}{|l|c|l|}
\hline \multicolumn{3}{|c|}{ VISCOMETER CONEIGURATION } \\
\hline Sensor & MV-IIP & Sensor Type \\
\hline Factor A & 3.76 & Pascals \\
\hline Factor $M$ & 4.40 & $1 /$ sec \\
\hline Temperature & 25.00 & OCelcius \\
\hline$\%$ D & 100.00 & Percent (\%) \\
\hline$\%$ Tau & 100.00 & Percent (\%) \\
\hline
\end{tabular}

\begin{tabular}{|c|c|}
\hline $\begin{array}{c}\text { SHEAR } \\
\text { RATE }\end{array}$ & $\begin{array}{l}\text { APPARENT } \\
\text { VISCOSITY }\end{array}$ \\
\hline$+50 \cdot \mathrm{Hz}$ & $2008.71 \cdot \mathrm{cP}$ \\
\hline$+100 \cdot \mathrm{Hz}$ & $1231.65 \cdot \mathrm{cP}$ \\
\hline$+200 \cdot \mathrm{Hz}$ & $740.51 \cdot \mathrm{cP}$ \\
\hline$+300 \cdot \mathrm{Hz}$ & $575.57 \cdot \mathrm{cP}$ \\
\hline$+400 \cdot \mathrm{Hz}$ & $470.09 \cdot \mathrm{cP}$ \\
\hline$-400 \cdot \mathrm{Hz}$ & $461.97 \cdot \mathrm{cP}$ \\
\hline$-300 \cdot \mathrm{Hz}$ & $498.87 \cdot \mathrm{cP}$ \\
\hline$-200 \cdot \mathrm{Hz}$ & $578.63 \cdot \mathrm{cP}$ \\
\hline$-100 \cdot \mathrm{Hz}$ & $801.35 \cdot \mathrm{cP}$ \\
\hline$-50 \cdot \mathrm{Hz}$ & $1182.40 \cdot \mathrm{cP}$ \\
\hline
\end{tabular}

\begin{tabular}{|l|c|c|c|}
\hline RHEOLOGICAL CLASSIFICATION & \multicolumn{4}{|c|}{ YIELD POHER IAW - PSEUDOPIASTIC } \\
\hline INITIAL YIELD STRESS & $T$ & 8.8201 & Pascals \\
\hline CONSISTENCY FACTOR & $\mathrm{K}$ & 12.6584 & Pascal-Sec \\
\hline NON-NEW TONIAN FLOW FACTOR & $n$ & 0.4519 & Dimensionless \\
\hline CURVE FIT ACCURACY & $\mathrm{R}^{2}$ & 94.1877 & Percent(\%) \\
\hline
\end{tabular}


ENERGY \& ENVIRONMENTAL RESEARCH CENTER

COAL BENEFICIATION PROJECT

Grand Forks, North Dakota 58202

SAMPLE INEORMATION

RHEOLOGY FOR THAILAND AUTOCLAVE SAMPLES

TEST\#: THAI.02 HWD @ $300 \mathrm{C}(-200$ mesh $)$

HIGH SOLIDS CONCENTRATION $=48.10 \mathrm{wt} \%$

\begin{tabular}{|l|l|l|l|l|l|l|l|}
\hline DATE $>$ Oct 31/95 & TIME> 17:04:53 & RATE 120 samples/minute
\end{tabular}

ARCHIVE FILE NAME>c:\123r3\THO24810.prn

\begin{tabular}{|c|c|c|c|}
\hline \multicolumn{4}{|c|}{ SOLIDS CONCENTRATION } \\
\hline Separate & 48.14 & 48.06 & Wt \% \\
\hline Average & \multicolumn{2}{|c|}{48.10} & Wt \% \\
\hline
\end{tabular}

\begin{tabular}{|l|c|c|c|}
\hline \multicolumn{3}{|c|}{ ASH CONCENTRATION } \\
\hline Separate & $* * * * *$ & $* * * * *$ & Wt \% \\
\hline Average & \multirow{2}{*}{$* * * * * *$} & Wt \% \\
\hline
\end{tabular}

\begin{tabular}{|l|c|l|}
\hline \multicolumn{2}{|c|}{ VISCOMETER CONEIGURATION } \\
\hline Sensor & MV-IIP & Sensor Type \\
\hline Eactor A & 3.76 & Pascals \\
\hline Factor M & 4.40 & $1 /$ sec \\
\hline Temperature & 25.00 & OCelcius \\
\hline$\%$ D & 100.00 & Percent (\%) \\
\hline$\%$ Tau & 70.00 & Percent (\%) \\
\hline
\end{tabular}

\begin{tabular}{|c|c|}
\hline $\begin{array}{c}\text { SHEAR } \\
\text { RATE }\end{array}$ & $\begin{array}{l}\text { APPARENT } \\
\text { VISCOSITY }\end{array}$ \\
\hline$+50 \cdot \mathrm{Hz}$ & $999.14 \cdot \mathrm{cP}$ \\
\hline$+100 \cdot \mathrm{Hz}$ & $757.37 \cdot \mathrm{cP}$ \\
\hline$+200 \cdot \mathrm{Hz}$ & $473.98 \cdot \mathrm{cP}$ \\
\hline$+300 \cdot \mathrm{Hz}$ & $373.72 \cdot \mathrm{cP}$ \\
\hline$+400 \cdot \mathrm{Hz}$ & $326.61 \cdot \mathrm{cP}$ \\
\hline$-400 \cdot \mathrm{Hz}$ & $353.27 \cdot \mathrm{cP}$ \\
\hline$-300 \cdot \mathrm{Hz}$ & $385.64 \cdot \mathrm{cP}$ \\
\hline$-200 \cdot \mathrm{Hz}$ & $438.67 \cdot \mathrm{cP}$ \\
\hline$-100 \cdot \mathrm{Hz}$ & $586.57 \cdot \mathrm{cP}$ \\
\hline$-50 \cdot \mathrm{Hz}$ & $863.15 \cdot \mathrm{cP}$ \\
\hline
\end{tabular}

\begin{tabular}{|l|c|c|c|}
\hline RHEOLOGICAL CLASSIFICATION & \multicolumn{3}{|c|}{ YIELD POWER LAN - PSEUDOPLASTIC } \\
\hline INITIAL YIELD STRESS & $\tau$ & 2.0497 & Pascals \\
\hline CONSISTENCY FACTOR & $\mathrm{K}$ & 4.9453 & Pascal-Sec $^{n}$ \\
\hline NON-NEWTONIAN FLOW FACTOR & $n$ & 0.5514 & Dimensionless \\
\hline CURVE FIT ACCURACY & $\mathrm{R}^{2}$ & 97.1625 & Percent(\%) \\
\hline
\end{tabular}


ENERGY \& ENVIRONMENTAL RESEARCH CENTER COAL BENEFICIATION PROJECT

Grand Forks, North Dakota 58202

\begin{tabular}{|c|c|c|}
\hline \multicolumn{3}{|c|}{$\begin{array}{l}\text { RHEOLOGY FOR THAILAND AUTOCLAVE SAMPLES } \\
\text { TEST\#: THAI.02 HWD @ } 300 \mathrm{C}(-200 \text { mesh) } \\
\text { MEDIUM SOLIDS CONCENTRATION }=47.06 \text { wt } \%\end{array}$} \\
\hline DATE $>$ Oct $31 / 95$ & TIME > $17: 19: 44$ & RATE $>120$ samples/minute \\
\hline
\end{tabular}

\begin{tabular}{|l|c|c|c|}
\hline \multicolumn{4}{|c|}{ SOLIDS CONCENTRATION } \\
\hline Separate & 47.09 & 47.02 & wt \% \\
\hline Average & \multicolumn{2}{|c|}{47.06} & Ht \% \\
\hline
\end{tabular}

\begin{tabular}{|c|c|}
\hline $\begin{array}{c}\text { SHEAR } \\
\text { RATE }\end{array}$ & $\begin{array}{c}\text { APPARENT } \\
\text { VISCOSITY }\end{array}$ \\
\hline$+50 \cdot \mathrm{Hz}$ & $540.86 \cdot \mathrm{cP}$ \\
\hline$+100 \cdot \mathrm{Hz}$ & $437.82 \cdot \mathrm{cP}$ \\
\hline $1200 \cdot \mathrm{Hz}$ & $308.75 \cdot \mathrm{cP}$ \\
\hline$+300 \cdot \mathrm{Hz}$ & $264.41 \cdot \mathrm{cP}$ \\
\hline$+400 \cdot \mathrm{Hz}$ & $243.50 \cdot \mathrm{cP}$ \\
\hline$-400 \cdot \mathrm{Hz}$ & $257.17 \cdot \mathrm{cP}$ \\
\hline$-300 \cdot \mathrm{Hz}$ & $284.01 \cdot \mathrm{cP}$ \\
\hline$-200 \cdot \mathrm{Hz}$ & $330.57 \cdot \mathrm{cP}$ \\
\hline$-100 \cdot \mathrm{Hz}$ & $487.96 \cdot \mathrm{cP}$ \\
\hline$-50 \cdot \mathrm{Hz}$ & $777.43 \cdot \mathrm{cP}$ \\
\hline
\end{tabular}

\begin{tabular}{|l|c|c|c|}
\hline \multicolumn{3}{|c|}{ ASH CONCENTRATION } \\
\hline Separate & $* * * * *$ & $* * * * *$ & Wt $\%$ \\
\hline Average & \multicolumn{2}{|c|}{$* * * * *$} & Wt $\%$ \\
\hline
\end{tabular}

\begin{tabular}{|l|c|l|}
\hline \multicolumn{2}{|c|}{ VISCOMETER CONFIGURATION } \\
\hline Sensor & MV-IIP & Sensor Type \\
\hline Factor A & 3.76 & Pascals \\
\hline Factor M & 4.40 & $1 /$ sec \\
\hline Temperature & 25.00 & 'Celcius \\
\hline$\%$ D & 100.00 & Percent (\%) \\
\hline$\%$ Tau & 40.00 & Percent (\%) \\
\hline
\end{tabular}

\begin{tabular}{|c|}
\hline $\begin{array}{c}\text { SAMPLE DENSITY } \\
(\text { grams/cm^3) }\end{array}$ \\
\hline$* * * * * *$ \\
\hline
\end{tabular}

\begin{tabular}{|l|c|c|l|}
\hline RHEOLOGICAL CLASSIFICATION & \multicolumn{3}{|c|}{ YIELD POWER LAW - PSEUDOPLASTIC } \\
\hline INITIAL YIELD STRESS & $\tau$ & 1.1828 & PasCals \\
\hline CONSISTENCY FACTOR & $R$ & 1.7922 & Pascal-Sec $^{n}$ \\
\hline NON-NEWTONIAN FLOW FACTOR & $n$ & 0.6659 & Dimensionless \\
\hline CURVE FIT ACCURACY & $\mathrm{R}^{2}$ & 99.1100 & Percent(\%) \\
\hline
\end{tabular}


ENERGY \& ENVIRONMENTAL RESEARCH CENTER COAL BENEFICIATION PROJECT

Grand Forks, North Dakota 58202

\begin{tabular}{|c|c|c|c|}
\hline \multicolumn{4}{|c|}{$\begin{array}{l}\text { RHEOLOGY FOR THAILAND AUTOCLAVE SAMPLES } \\
\text { TEST\#: THAI.O2 HWD @ } 300 \text { C ( }-200 \text { mesh }) \\
\text { LOW SOLIDS CONCENTRATION }=46.00 \text { w } \%\end{array}$} \\
\hline DATE $>$ Oct $31 / 95$ & TIME> $17: 30: 18$ & RATE $\rangle 120$ & samples/minute \\
\hline
\end{tabular}

\begin{tabular}{|c|c|c|c|}
\hline \multicolumn{4}{|c|}{ SOLIDS CONCENTRATION } \\
\hline Separate & 45.96 & 46.04 & Wt $\%$ \\
\hline Average & \multicolumn{2}{|c|}{46.00} & Wt $\%$ \\
\hline
\end{tabular}

\begin{tabular}{|l|c|c|c|}
\hline \multicolumn{3}{|c|}{ ASH CONCENTRATION } \\
\hline Separate & $* * * * *$ & $* * * * *$ & Wt \% \\
\hline Average & ***** & Wt \% \\
\hline
\end{tabular}

\begin{tabular}{|l|c|l|}
\hline \multicolumn{3}{|c|}{ VISCOMETER CONEIGURATION } \\
\hline Sensor & MV-IIP & Sensor Type \\
\hline Factor A & 3.76 & Pascals \\
\hline Factor M & 4.40 & $1 /$ sec \\
\hline Temperature & 25.00 & Celcius \\
\hline$\%$ D & 100.00 & Percent (\%) \\
\hline$\%$ Tau & 20.00 & Percent (\%) \\
\hline
\end{tabular}

\begin{tabular}{|c|c|}
\hline $\begin{array}{c}\text { SHEAR } \\
\text { RATE }\end{array}$ & $\begin{array}{l}\text { APPARENT } \\
\text { VISCOSITY }\end{array}$ \\
\hline$+50 \cdot \mathrm{Hz}$ & $273.52 \cdot \mathrm{cP}$ \\
\hline$+100 \cdot \mathrm{Hz}$ & $207.50 \cdot \mathrm{cP}$ \\
\hline$+200 \cdot \mathrm{Hz}$ & $152.75 \cdot \mathrm{cP}$ \\
\hline$+300 \cdot \mathrm{Hz}$ & $132.67 \cdot \mathrm{cP}$ \\
\hline$+400 \cdot \mathrm{Hz}$ & $122.60 \cdot \mathrm{cP}$ \\
\hline$-400 \cdot \mathrm{Hz}$ & $127.83 \cdot \mathrm{cP}$ \\
\hline$-300 \cdot \mathrm{Hz}$ & $144.53 \cdot \mathrm{cP}$ \\
\hline$-200 \cdot \mathrm{Hz}$ & $172.97 \cdot \mathrm{cP}$ \\
\hline$-100 \cdot \mathrm{Hz}$ & $238.79 \cdot \mathrm{cP}$ \\
\hline$-50 \cdot \mathrm{Hz}$ & $361.37 \cdot \mathrm{cP}$ \\
\hline
\end{tabular}

\begin{tabular}{|l|c|c|l|}
\hline RHEOLOGICAL CLASSIFICATION & \multicolumn{3}{|l|}{ PSEUDOPLASTIC } \\
\hline INITIAL YIELD STRESS & $\tau$ & 0.1294 & Pascals \\
\hline CONSISTENCY EACTOR & $R$ & 1.0774 & Pascal-Sec $^{n}$ \\
\hline NON-NEWTONIAN FLOW FACTOR & $n$ & 0.6346 & Dimensionless \\
\hline CURVE EIT ACCURACY & $\mathrm{R}^{2}$ & 99.6560 & Percent(\%) \\
\hline
\end{tabular}


ENERGY \& ENVIRONMENTAL RESEARCH CENTER

COAL BENEFICIATION PROJECT

Grand Forks, North Dakota 58202

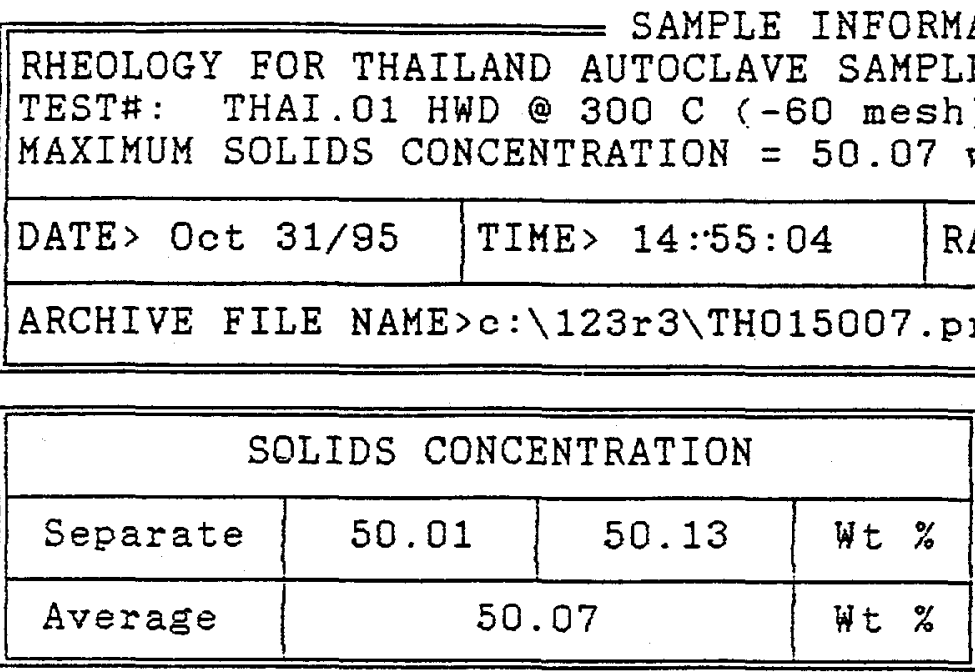

\begin{tabular}{|l|c|c|c|}
\hline \multicolumn{3}{|c|}{ ASH CONCENTRATION } \\
\hline Separate & $* * * * *$ & $* * * * *$ & wt $\%$ \\
\hline Average & ****** & wt \% \\
\hline
\end{tabular}

\begin{tabular}{|l|c|l|}
\hline \multicolumn{3}{|c|}{ VISCOMETER CONFIGURATION } \\
\hline Sensor & MV-IIP & Sensor Type \\
\hline Eactor A & 3.76 & Pascals \\
\hline Factor M & 4.40 & $1 /$ sec \\
\hline Temperature & 25.00 & Celcius \\
\hline$\%$ D & 100.00 & Percent (\%) \\
\hline$\%$ Tau & 100.00 & Percent (\%) \\
\hline
\end{tabular}

\begin{tabular}{|c|c|}
\hline $\begin{array}{c}\text { SHEAR } \\
\text { RATE }\end{array}$ & $\begin{array}{l}\text { APPARENT } \\
\text { VISCOSITY }\end{array}$ \\
\hline$+50 \cdot \mathrm{Hz}$ & $2142.81 \cdot \mathrm{cP}$ \\
\hline$+100 \cdot \mathrm{Hz}$ & $1279.11 \cdot \mathrm{cP}$ \\
\hline$+200 \cdot \mathrm{Hz}$ & $825.41 \cdot \mathrm{cP}$ \\
\hline$+300 \cdot \mathrm{Hz}$ & $655.39 \cdot \mathrm{cP}$ \\
\hline$+400 \cdot \mathrm{Hz}$ & $525.37 \cdot \mathrm{cP}$ \\
\hline$-400 \cdot \mathrm{Hz}$ & $477.63 \cdot \mathrm{cP}$ \\
\hline$-300 \cdot \mathrm{Hz}$ & $457.23 \cdot \mathrm{cP}$ \\
\hline$-200 \cdot \mathrm{Hz}$ & $469.25 \cdot \mathrm{cP}$ \\
\hline$-100 \cdot \mathrm{Hz}$ & $562.80 \cdot \mathrm{cP}$ \\
\hline$-50 \cdot \mathrm{Hz}$ & $756.53 \cdot \mathrm{cP}$ \\
\hline
\end{tabular}

\begin{tabular}{|l|c|c|c|c|}
\hline RHEOLOGICAL CLASSIFICATION & \multicolumn{3}{|c|}{ YIELD POWER LAW - PSEUDOPLASTIC } \\
\hline INITIAI YIELD STRESS & $\tau$ & 13.3740 & PasCaIs \\
\hline CONSISTENCY EACTOR & $\mathrm{K}$ & 12.1751 & PasCal-Sec \\
\hline NON-NEATONIAN ELOW FACTOR & $n$ & 0.4744 & Dimensionless \\
\hline CIRVE FIT ACCURACY & $\mathrm{R}^{2}$ & 95.8465 & Percent(\%) \\
\hline
\end{tabular}


ENERGY \& ENVTRONMENTAL FESEARCH CENTER

COAL BENEFTCIATION PROJECT

Grand Forks, North Dakota 58202

SAMPLE INEORMATION

RHEOLOGY FOR THAILAND AUTOCLAVE SAMPLES

TEST\#: THAI.01 HWD @ $300 \mathrm{C}(-60$ mesh $)$

HIGH SOLIDS CONCENTRATION $=49.58$ wt

\begin{tabular}{|c|c|c|}
\hline DATE> Oot $31 / 95$ & TIME> $14: 39: 51$ & RATE> 60 samples/minute \\
\hline
\end{tabular}

ARCHIVE FILE NAME>c: \123r3\TH014958.prn

\begin{tabular}{|l|c|c|c|}
\hline \multicolumn{4}{|c|}{ SOLIDS CONCENTRATION } \\
\hline Separate & 49.64 & 49.51 & Ht \% \\
\hline Average & 49.57 & Wt \% \\
\hline
\end{tabular}

\begin{tabular}{|l|c|c|c|}
\hline \multicolumn{3}{|c|}{ ASH CONCENTRATION } \\
\hline Separate & ******* & $* * * * * * *$ & Ht \% \\
\hline Average & ****** & Wt \% \\
\hline
\end{tabular}

\begin{tabular}{|l|c|l|}
\hline \multicolumn{2}{|c|}{ VISCOMETER CONFIGURATION } \\
\hline Sensor & MV-IIP & Sensor Type \\
\hline Eactor A & 3.76 & Pascals \\
\hline Factor M & 4.40 & $1 /$ sec \\
\hline Temperature & 25.00 & ${ }^{\circ}$ Celcius \\
\hline$\%$ D & 100.00 & Percent $(\%)$ \\
\hline$\%$ Tau & 100.00 & Percent $(\%)$ \\
\hline
\end{tabular}

\begin{tabular}{|c|c|}
\hline $\begin{array}{c}\text { SHEAR } \\
\text { RATE }\end{array}$ & $\begin{array}{l}\text { APPARENT } \\
\text { VISCOSITY }\end{array}$ \\
\hline$+50 \cdot \mathrm{Hz}$ & $1778.69 \cdot \mathrm{cP}$ \\
\hline$+100 \cdot \mathrm{Hz}$ & $982.54 \cdot \mathrm{cP}$ \\
\hline$+200 \cdot \mathrm{Hz}$ & $618.68 \cdot \mathrm{cP}$ \\
\hline$+300 \cdot \mathrm{Hz}$ & $506.39 \cdot \mathrm{cP}$ \\
\hline$+100 \cdot \mathrm{Hz}$ & $431.48 \cdot \mathrm{cP}$ \\
\hline$-400 \cdot \mathrm{Hz}$ & $417.77 \cdot \mathrm{cP}$ \\
\hline$-300 \cdot \mathrm{Hz}$ & $405.63 \cdot \mathrm{cP}$ \\
\hline$-200 \cdot \mathrm{Hz}$ & $420 . \mathrm{E} 2 \cdot \mathrm{cF}$ \\
\hline$-100 \cdot \mathrm{Hz}$ & $502.92 \cdot \mathrm{cP}$ \\
\hline$-50 \cdot \mathrm{Hz}$ & $677.14 \cdot \mathrm{cP}$ \\
\hline
\end{tabular}

\begin{tabular}{|l|c|c|c|}
\hline RHEOLOGICAL CLASSIFICATION & \multicolumn{3}{|c|}{ YIELD POWER LAW - PSEUDOPLASTIC } \\
\hline INITIAL YIELD STRESS & $\tau$ & 8.2495 & Pascals \\
\hline CONSISTENCY FACTOR & $\mathrm{K}$ & 10.0451 & Pascal-Sec $^{n}$ \\
\hline NON-NEWTONIAN FLOW FACTOR & $n$ & 0.4668 & Dimensionless \\
\hline CURYE EIT ACCURACY & $\mathrm{R}^{2}$ & 95.5338 & Percent(\%) \\
\hline
\end{tabular}


ENERGY \& ENVIRONMENTAL RESEARCH CENTER

COAL BENEFICIATION PROJECT

Grand Forks, North Dakota 58202

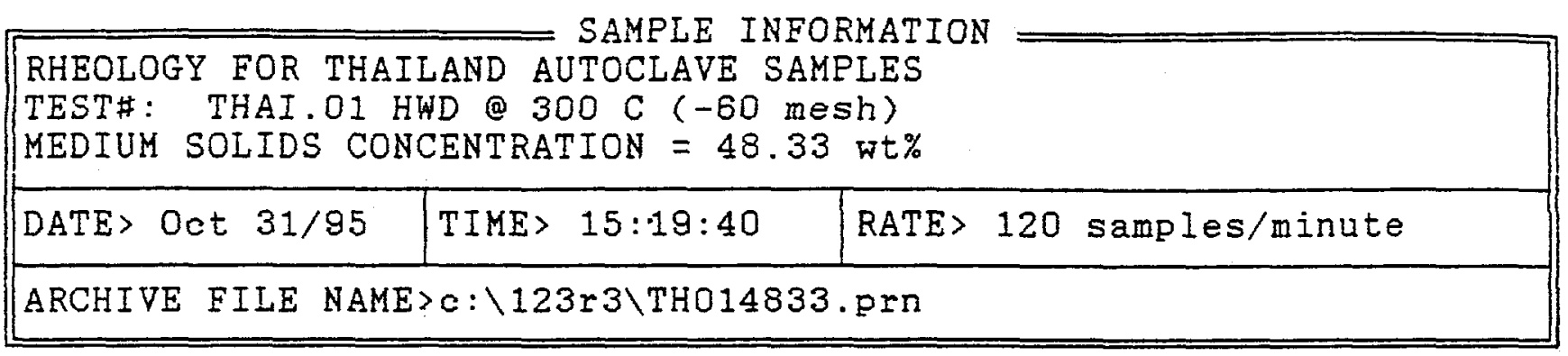

\begin{tabular}{|c|c|c|c|}
\hline \multicolumn{4}{|c|}{ SOLIDS CONCENTRATION } \\
\hline Separate & 48.30 & 48.36 & Ht \% \\
\hline Average & \multicolumn{2}{|c|}{48.33} & Wt \% \\
\hline
\end{tabular}

\begin{tabular}{|c|c|c|c|}
\hline \multicolumn{4}{|c|}{ ASH CONCENTRATION } \\
\hline$\because \because \cdots=5$ & 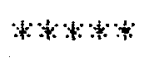 & $* * * * *$ & Wt \% \\
\hline Average & \multicolumn{2}{|c|}{$* * * * *$} & Wt \% \\
\hline
\end{tabular}

\begin{tabular}{|c|c|}
\hline $\begin{array}{l}\text { SHEAR } \\
\text { RATE }\end{array}$ & $\begin{array}{l}\text { APPARENT } \\
\text { VISCOSITY }\end{array}$ \\
\hline$+\quad 50 \cdot \mathrm{Hz}$ & $720.39 \cdot \mathrm{cP}$ \\
\hline$+100 \cdot \mathrm{Hz}$ & $582.91 \cdot \mathrm{cP}$ \\
\hline$+200 \cdot \mathrm{Hz}$ & $412.95 \cdot \mathrm{cP}$ \\
\hline$-920 \cdot \%=$ & $\theta=07=-$ \\
\hline$+400 \cdot \mathrm{Hz}$ & $318.73 \cdot \mathrm{cP}$ \\
\hline$-400 \cdot \mathrm{Hz}$ & $306.18 \cdot \mathrm{cP}$ \\
\hline$-300 \cdot \mathrm{Hz}$ & $298.89 \cdot c P$ \\
\hline$-200 \cdot \mathrm{Hz}$ & $308.97 \cdot \mathrm{cP}$ \\
\hline$-100 \cdot \mathrm{Hz}$ & $361.48 \cdot c P$ \\
\hline$-\quad 50 \cdot \mathrm{Hz}$ & $462.20 \cdot c P$ \\
\hline
\end{tabular}

\begin{tabular}{|l|c|l|}
\hline \multicolumn{3}{|c|}{ VISCOMETER CONFIGURATION } \\
\hline Sensor & MV-IIP & Sensor Type \\
\hline Factor A & 3.76 & Pascals \\
\hline Eactor M & 4.40 & $1 /$ sec \\
\hline Temperature & 25.00 & 'Celcius \\
\hline$\%$ D & 100.00 & Percent (\%) \\
\hline$\%$ Tau & 60.00 & Percent (\%) \\
\hline
\end{tabular}

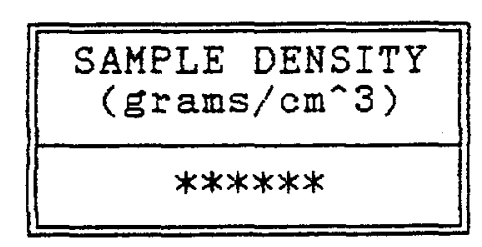

\begin{tabular}{|l|c|c|c|}
\hline RHEOLOGICAL CLASSIFICATION & \multicolumn{3}{|c|}{ YIELD POWER LAW - PSEUDOPLASTIC } \\
\hline INITIAL YIELD STRESS & $T$ & 1.5739 & Pascals \\
\hline CONSISTENCY FACTOR & $\mathrm{K}$ & 2.7049 & Pascal-Sec $^{n}$ \\
\hline NON-NEWTONIAN FLOW FACTOR & $n$ & 0.6434 & Dimensionless \\
\hline CURVE FIT ACCURACY & $\mathrm{R}^{2}$ & 99.1138 & Percent $(\%)$ \\
\hline
\end{tabular}


ENERGY \& ENVIRONMENTAL RESEARCH CENTER COAL BENEFICIATION PROJECT

Grand Forks, North Dakota 58202

\begin{tabular}{|c|c|c|}
\hline \multicolumn{3}{|c|}{$\begin{array}{l}\text { RHEOLOGY FOR THAILAND AUTOCLAVE SAMPLES } \\
\text { TESTH: THAI.01 HWD @ } 300 \mathrm{C}(-60 \mathrm{mesh}) \\
\text { LOW SOLIDS CONCENTRATION }=46.34 \mathrm{wt} \%\end{array}$} \\
\hline DATE $>$ Oct $31 / 95$ & TIME $>15: 33: 56$ & RATE> 120 samples/minute \\
\hline
\end{tabular}

\begin{tabular}{|c|c|c|c|}
\hline \multicolumn{4}{|c|}{ SOLIDS CONCENTRATION } \\
\hline Separate & 46.38 & 46.30 & Wt $\%$ \\
\hline Average & \multicolumn{2}{|c|}{46.34} & Wt $\%$ \\
\hline
\end{tabular}

\begin{tabular}{|l|c|c|c|}
\hline \multicolumn{3}{|c|}{ ASH CONCENTRATION } \\
\hline Separate & $* * * * *$ & $* * * * *$ & wt \% \\
\hline Average & ****** & wt \% \\
\hline
\end{tabular}

\begin{tabular}{|l|c|l|}
\hline \multicolumn{3}{|c|}{ VISCOMETER CONFIGURATION } \\
\hline Sensor & MV-IIP & Sensor Type \\
\hline Factor A & 3.76 & Pascals \\
\hline Factor M & 4.40 & $1 /$ sec \\
\hline Temperature & 25.00 & ${ }^{\circ}$ Celcius \\
\hline$\%$ D & 100.00 & Percent (\%) \\
\hline$\%$ Tau & 20.00 & Percent (\%) \\
\hline
\end{tabular}

\begin{tabular}{|c|c|}
\hline $\begin{array}{c}\text { SHEAR } \\
\text { RATE }\end{array}$ & $\begin{array}{l}\text { APPARENT } \\
\text { VISCOSITY }\end{array}$ \\
\hline$+50 \cdot \mathrm{Hz}$ & $218.79 \cdot \mathrm{cP}$ \\
\hline$+100 \cdot \mathrm{Hz}$ & $183.79 \cdot \mathrm{cP}$ \\
\hline$+200 \cdot \mathrm{Hz}$ & $153.35 \cdot \mathrm{cP}$ \\
\hline$+300 \cdot \mathrm{Hz}$ & $143.11 \cdot \mathrm{cP}$ \\
\hline$+400 \cdot \mathrm{Hz}$ & $136.45 \cdot \mathrm{cP}$ \\
\hline$-400 \cdot \mathrm{Hz}$ & $139.98 \cdot \mathrm{cP}$ \\
\hline$-300 \cdot \mathrm{Hz}$ & $140.27 \cdot \mathrm{cP}$ \\
\hline$-200 \cdot \mathrm{Hz}$ & $146.28 \cdot \mathrm{cP}$ \\
\hline$-100 \cdot \mathrm{Hz}$ & $172.02 \cdot \mathrm{cP}$ \\
\hline$-50 \cdot \mathrm{Hz}$ & $227.77 \cdot \mathrm{cP}$ \\
\hline
\end{tabular}

\begin{tabular}{|l|c|c|c|}
\hline RHEOLOGICAL CLASSIEICATION & \multicolumn{2}{|c|}{ PSEUDOPLASTIC } \\
\hline INITIAL YIELD STRESS & $\tau$ & 0.0000 & Pascals \\
\hline CONSISTENCY EACTOR & $R$ & 0.6248 & Pascal.Secn \\
\hline NON-NEWTONIAN ELOH FACTOR & $n$ & 0.7449 & DimensionIEsS \\
\hline CURVE EIT ACCURACY & $R^{2}$ & 99.8903 & Percent(\%) \\
\hline
\end{tabular}


ENERGY \& ENVIRONMENTAL RESEARCH CENTER

COAL BENEFICIATION PROJECT

Grand Forks, North Dakota 58202

\section{RHEOLOGY FOR THAILAND AUTOCLAVE SAMPLES}

TEST\#: RAW COAL ( -60 mesh)

MAXIMUM SOLIDS CONCENTRATION $=37.66 \mathrm{wt} \%$

\begin{tabular}{|l|l|l|l|l|l|l|}
\hline DATE $>$ Nov 01/95 & TIME $>12: 21: 31$ & RATE> 120 samples/minute \\
\hline
\end{tabular}

ARCHIVE FILE NAME>c: \123r3\THRW3766.prn

\begin{tabular}{|c|c|c|c|}
\hline \multicolumn{4}{|c|}{ SOLIDS CONCENTRATION } \\
\hline Separate & 37.64 & 37.68 & Ht \% \\
\hline Average & \multicolumn{2}{|c|}{37.66} & W七 \% \\
\hline
\end{tabular}

\begin{tabular}{|l|c|c|c|}
\hline \multicolumn{3}{|c|}{ ASH CONCENTRATION } \\
\hline Separate & $* * * * *$ & $* * * * *$ & wt $\%$ \\
\hline Average & ****** & Ht \% \\
\hline
\end{tabular}

\begin{tabular}{|l|c|l|}
\hline \multicolumn{3}{|c|}{ VISCOMETER CONFIGURATION } \\
\hline Sensor & MV-IIP & Sensor Type \\
\hline Factor A & 3.76 & Pascals \\
\hline Factor M & 4.40 & $1 /$ sec \\
\hline Temperature & 25.00 & 'Celcius \\
\hline$\%$ D & 100.00 & Percent (\%) \\
\hline$\%$ Tau & 100.00 & Percent (\%) \\
\hline
\end{tabular}

\begin{tabular}{|c|c|}
\hline $\begin{array}{c}\text { SHEAR } \\
\text { RATE }\end{array}$ & $\begin{array}{l}\text { APPARENT } \\
\text { VISCOSITY }\end{array}$ \\
\hline$+50 \cdot \mathrm{Hz}$ & $1741.18 \cdot \mathrm{cP}$ \\
\hline$+100 \cdot \mathrm{Hz}$ & $1041.60 \cdot \mathrm{cP}$ \\
\hline$+200 \cdot \mathrm{Hz}$ & $602.90 \cdot \mathrm{cP}$ \\
\hline$+300 \cdot \mathrm{Hz}$ & $455.65 \cdot \mathrm{cP}$ \\
\hline$+400 \cdot \mathrm{Hz}$ & $377.05 \cdot \mathrm{cP}$ \\
\hline$-400 \cdot \mathrm{Hz}$ & $357.19 \cdot \mathrm{cP}$ \\
\hline$-300 \cdot \mathrm{Hz}$ & $351.23 \cdot \mathrm{cP}$ \\
\hline$-200 \cdot \mathrm{Hz}$ & $372.32 \cdot \mathrm{cP}$ \\
\hline$-100 \cdot \mathrm{Hz}$ & $454.62 \cdot \mathrm{cP}$ \\
\hline$-50 \cdot \mathrm{Hz}$ & $613.42 \cdot \mathrm{cP}$ \\
\hline
\end{tabular}

\begin{tabular}{|l|c|r|l|}
\hline RHEOLOGICAL CLASSIFICATION & \multicolumn{4}{|c|}{ YIELD POWER LAW - PSEUDOPLASTIC } \\
\hline INITIAL YIELD STRESS & $T$ & 15.4387 & Pascals \\
\hline CONSISTENCY FACTOR & $\mathrm{K}$ & 13.9183 & Pascal•Sec $^{n}$ \\
\hline NON-NEWTONIAN FLOH FACTOR & $n$ & 0.3829 & Dimensionless \\
\hline CURVE FIT ACCURACY & $\mathrm{R}^{2}$ & 95.7772 & Percent(\%) \\
\hline
\end{tabular}


ENERGY \& ENVIRONMENTAL RESEARCH CENTER

COAL BENEFICIATION PROJECT

Grand Forks, North Dakota 58202

SAMPLE INEORMATION

RHEOLOGY FOR THAILAND AUTOCLAVE SAMPLES

TEST\#: RAW COAL ( -60 mesh)

HIGH SOLIDS CONCENTRATION $=36.59$ wt $\%$

\begin{tabular}{|l|l|l|l|l|}
\hline DATE $>$ Nov 01/95 & TIME> 12:34:51 & RATE> 120 samples/minute
\end{tabular}

ARCHIVE FILE NAME>c: \123r3\THRW3659.prn

\begin{tabular}{|c|c|c|c|}
\hline \multicolumn{4}{|c|}{ SOLIDS CONCENTRATION } \\
\hline Separate & 36.60 & 36.57 & Wt \% \\
\hline Average & \multicolumn{2}{|c|}{36.59} & wt \% \\
\hline
\end{tabular}

ASH CONCENTRATION

\begin{tabular}{|l|c|c|c|}
\hline Separate & $* * * * *$ & $* * * * *$ & Wt \% \\
\hline Average & \multicolumn{2}{|c|}{$* * * * *$} & Ht \% \\
\hline
\end{tabular}

\begin{tabular}{|l|c|l|}
\hline \multicolumn{2}{|c|}{ VISCOMETER CONFIGURATION } \\
\hline Sensor & MV-IIP & Sensor Type \\
\hline Eactor A & 3.76 & Pascals \\
\hline Factor M & 4.40 & $1 /$ sec \\
\hline Temperature & 25.00 & ${ }^{\circ}$ Celcius \\
\hline$\%$ D & 100.00 & Percent (\%) \\
\hline$\%$ Tau & 50.00 & Percent $(\%)$ \\
\hline
\end{tabular}

\begin{tabular}{|c|c|}
\hline $\begin{array}{c}\text { SHEAR } \\
\text { RATE }\end{array}$ & $\begin{array}{c}\text { APPARENT } \\
\text { VISCOSITY }\end{array}$ \\
\hline$+50 \cdot \mathrm{Hz}$ & $798.03 \cdot \mathrm{cP}$ \\
\hline$+100 \cdot \mathrm{Hz}$ & $603.13 \cdot \mathrm{cP}$ \\
\hline$+200 \cdot \mathrm{Hz}$ & $452.64 \cdot \mathrm{cP}$ \\
\hline$+300 \cdot \mathrm{Hz}$ & $387.46 \cdot \mathrm{cP}$ \\
\hline$+400 \cdot \mathrm{Hz}$ & $325.29 \cdot \mathrm{cP}$ \\
\hline$-400 \cdot \mathrm{Hz}$ & $310.75 \cdot \mathrm{cP}$ \\
\hline$-300 \cdot \mathrm{Hz}$ & $306.55 \cdot \mathrm{cP}$ \\
\hline$-200 \cdot \mathrm{Hz}$ & $333.36 \cdot \mathrm{cP}$ \\
\hline$-100 \cdot \mathrm{Hz}$ & $422.25 \cdot \mathrm{cP}$ \\
\hline$-50 \cdot \mathrm{Hz}$ & $591.06 \cdot \mathrm{cP}$ \\
\hline
\end{tabular}

\begin{tabular}{|c|c|c|c|}
\hline RHEOLOGICAL CLASSIFICATION & \multicolumn{3}{|c|}{ PSEUDOPLASTIC } \\
\hline INITIAL YIELD STRESS & $T$ & 0.3074 & Pascals \\
\hline CONSISTENCY FACTOR & $\mathrm{K}$ & 3.1680 & Pascal-Secn \\
\hline NON-NEWTONIAN FLOW FACTOR & $n$ & 0.6284 & Dimensionless \\
\hline CURVE FIT ACCURACY & $\mathrm{R}^{2}$ & 99.4036 & Percent $(\%)$ \\
\hline
\end{tabular}


ENERGY \& ENVIRONMENTAL RESEARCH CENTER

COAL BENEEICIATION PROJECT

Grand Forks, North Dakota 58202

SAMPLE INFORMATION

RHEOLOGY FOR THAILAND AUTOCLAVE SAMPLES

TEST\#: RAW COAL ( -60 mesh)

MEDIUM SOLIDS CONCENTRATION $=35.54$ wt $\%$

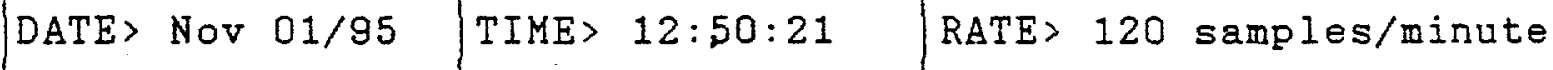

ARCHIVE FILE NAME>C: \123r3\THRW3554.prn

\begin{tabular}{|l|c|c|c|}
\hline \multicolumn{3}{|c|}{ SOLIDS CONCENTRATION } \\
\hline Separate & 35.56 & 35.52 & Wt \% \\
\hline Average & 35.54 & Ht \% \\
\hline
\end{tabular}

\begin{tabular}{|l|c|c|c|}
\hline \multicolumn{3}{|c|}{ ASH CONCENTRATION } \\
\hline Separate & $* * * * * *$ & $* * * * * *$ & Wt $\%$ \\
\hline Average & $* * * * *$ & Wt $\%$ \\
\hline
\end{tabular}

\begin{tabular}{|l|c|l|}
\hline \multicolumn{2}{|c|}{ VISCOMETER CONFIGURATION } \\
\hline Sensor & MV-IIP & Sensor Type \\
\hline Factor A & 3.76 & Eascals \\
\hline Eactor M & 4.40 & $1 /$ sec \\
\hline Temperature & 25.00 & Oelcius \\
\hline$\%$ D & 100.00 & Percent (\%) \\
\hline$\%$ Tau & 30.00 & Percent (\%) \\
\hline
\end{tabular}

\begin{tabular}{|c|c|}
\hline $\begin{array}{c}\text { SHEAR } \\
\text { RATE }\end{array}$ & $\begin{array}{l}\text { APPARENT } \\
\text { VISCOSITY }\end{array}$ \\
\hline $50 \cdot \mathrm{Hz}$ & S65.56.0P \\
\hline$+100 \cdot \mathrm{Hz}$ & $285.81 \cdot \mathrm{OP}$ \\
\hline$+200 \cdot \mathrm{Hz}$ & $227.94 \cdot \mathrm{cP}$ \\
\hline$+300 \cdot \mathrm{Hz}$ & $207.22 \cdot \mathrm{cP}$ \\
\hline$+400 \cdot \mathrm{Hz}$ & $188.88 \cdot \mathrm{cP}$ \\
\hline$-400 \cdot \mathrm{Hz}$ & $187.86 \cdot \mathrm{cP}$ \\
\hline$-300 \cdot \mathrm{Hz}$ & $180.12 \cdot \mathrm{cP}$ \\
\hline$-200 \cdot \mathrm{Hz}$ & $205.90 \cdot \mathrm{cP}$ \\
\hline$-100 \cdot \mathrm{Hz}$ & $257.80 \cdot \mathrm{cP}$ \\
\hline$-50 \cdot \mathrm{Hz}$ & $366.06 \cdot \mathrm{cP}$ \\
\hline
\end{tabular}

\begin{tabular}{|l|c|c|c|}
\hline RHEOLOGICAL CLASSIFICATION & \multicolumn{3}{|c|}{ PSEUDOPLASTIC } \\
\hline INITIAL YIELD STRESS & $T$ & 0.0000 & Pascals \\
\hline CONSISTENCY FACTOR & $K$ & 1.2009 & Pascal-Sec \\
\hline NON-NEWTONIAN FLOW FACTOR & $n$ & 0.6907 & Dimensionless \\
\hline CURVE FIT ACCURACY & $R^{2}$ & 99.8437 & Percent $\%)$ \\
\hline
\end{tabular}


ENERGY \& ENVIRONMENTAL RESEARCH CENTER

COAL BENEEICIATION PROJECT

Grand Forks, North Dakota 58202

\section{- SAMPLE INFORMATION \\ RHEOLOGY FOR THAILAND AUTOCLAVE SAMPLES \\ TEST\# : RAW COAL ( -60 mesh) \\ LOW SOLIDS CONCENTRATION $=34.41 \mathrm{wt} \%$}

\begin{tabular}{|l|l|l|l|l|l|l|}
\hline DATE $>$ Nov 01/95 & TIME $>13: 01: 22$ & RATE $>120$ samples/minute
\end{tabular}

ARCHIVE FILE NAME>c: \123r3\THRH $3441 . \mathrm{prn}$

\begin{tabular}{|l|c|c|c|}
\hline \multicolumn{4}{|c|}{ SOLIDS CONCENTRATION } \\
\hline Separate & 34.44 & 34.37 & Wt \% \\
\hline Average & \multicolumn{2}{|c|}{34.41} & Wt \% \\
\hline
\end{tabular}

\begin{tabular}{|c|c|c|c|}
\hline \multicolumn{4}{|c|}{ ASH CONCENTRATION } \\
\hline Separate & $* * * * * *$ & $* * * * * * *$ & $W t \%$ \\
\hline Averase & \multicolumn{2}{|c|}{$* * * * * * * *$} & Wt \% \\
\hline
\end{tabular}

\begin{tabular}{|l|c|l|}
\hline \multicolumn{3}{|c|}{ VISCOMETER CONEIGURATION } \\
\hline Sensor & MV-IIP & Sensor Type \\
\hline Factor A & 3.76 & Pascals \\
\hline Factor M & 4.40 & $1 /$ sec \\
\hline Temperature & 25.00 & 'Celcius \\
\hline$\%$ D & 100.00 & Percent (\%) \\
\hline$\%$ Tau & 20.00 & Percent (\%) \\
\hline
\end{tabular}

\begin{tabular}{|c|c|}
\hline $\begin{array}{c}\text { SHEAR } \\
\text { RATE }\end{array}$ & $\begin{array}{l}\text { APPARENT } \\
\text { VISCOSITY }\end{array}$ \\
\hline$+50 \cdot \mathrm{Hz}$ & $164.26 \cdot \mathrm{cP}$ \\
\hline$+100 \cdot \mathrm{Hz}$ & $136.50 \cdot \mathrm{cP}$ \\
\hline$+200 \cdot \mathrm{Hz}$ & $115.48 \cdot \mathrm{cP}$ \\
\hline$+300 \cdot \mathrm{Hz}$ & $100.76 \cdot \mathrm{cP}$ \\
\hline$+400 \cdot \mathrm{Hz}$ & $97.48 \cdot \mathrm{cP}$ \\
\hline$-400 \cdot \mathrm{Hz}$ & $101.07 \cdot \mathrm{cP}$ \\
\hline$-300 \cdot \mathrm{Hz}$ & $108.59 \cdot \mathrm{cP}$ \\
\hline$-200 \cdot \mathrm{Hz}$ & $118.72 \cdot \mathrm{cP}$ \\
\hline$-100 \cdot \mathrm{Hz}$ & $147.04 \cdot \mathrm{cP}$ \\
\hline$-50 \cdot \mathrm{Hz}$ & $206.22 \cdot \mathrm{cP}$ \\
\hline
\end{tabular}

\begin{tabular}{|l|c|c|l|}
\hline RHEOLOGICAL CLASSIEICATION & \multicolumn{2}{|l|}{ PSEUDOFLASTIC } \\
\hline INITIAL YIELD STRESS & $T$ & 0.1202 & Pascals \\
\hline CONSISTENCY FACTOR & $\mathrm{K}$ & 0.4409 & Pascal-Sec \\
\hline NON-NENTONIAN FLOH FACTOR & $n$ & 0.7452 & Dimensionless \\
\hline CURVE FIT ACCURACY & $\mathrm{R}^{2}$ & 99.9463 & Percent $(\%)$ \\
\hline
\end{tabular}


ENERGY \& ENVIRONMENTAL RESEARCH CENTER

COAL BENEFICIATION PROJECT

Grand Forks, North Dakota 58202

RHEOLOGY FOR THAILAND AUTOCLAVE SAMPLES

TEST\#: RAW COAL -1/4"x 20 mesh pulverized to -60 mesh MAXIMUM SOLIDS CONCENTRATION $=39.24$ wt $\%$

\begin{tabular}{|l|l|l|l|l|l|l|}
\hline DATE> Nov 07/95 & TIME> 11:21:07 & RATE> 120 samples/minute
\end{tabular}

ARCHIVE FILE NAME>c: \123r3\THRW3924.prn

\begin{tabular}{|c|c|c|c|}
\hline \multicolumn{4}{|c|}{ SOLIDS CONCENTRATION } \\
\hline Separate & 39.29 & 39.19 & Wt \% \\
\hline Average & \multicolumn{2}{|c|}{39.24} & H \\
\hline
\end{tabular}

\begin{tabular}{|l|c|c|c|}
\hline \multicolumn{3}{|c|}{ ASH CONCENTRATION } \\
\hline Separate & $* * * * *$ & $* * * * *$ & Wt \% \\
\hline Average & $* * * * * *$ & Wt \% \\
\hline
\end{tabular}

\begin{tabular}{|l|c|l|}
\hline \multicolumn{3}{|c|}{ VISCOMETER CONFIGURATION } \\
\hline Sensor & MV-IIP & Sensor Type \\
\hline Factor A & 3.76 & Pascals \\
\hline Factor M & 4.40 & $1 /$ sec \\
\hline Temperature & 25.00 & OCelcius \\
\hline$\%$ D & 100.00 & Percent (\%) \\
\hline$\%$ Tau & 100.00 & Percent (\%) \\
\hline
\end{tabular}

\begin{tabular}{|c|c|}
\hline $\begin{array}{c}\text { SHEAR } \\
\text { RATE }\end{array}$ & $\begin{array}{l}\text { APPARENT } \\
\text { VISCOSITY }\end{array}$ \\
\hline$+50 \cdot \mathrm{Hz}$ & $2962.10 \cdot \mathrm{cP}$ \\
\hline$+100 \cdot \mathrm{Hz}$ & $1734.00 \cdot \mathrm{cP}$ \\
\hline$+200 \cdot \mathrm{Hz}$ & $1073.56 \cdot \mathrm{cP}$ \\
\hline$+300 \cdot \mathrm{Hz}$ & $730.61 \cdot \mathrm{cP}$ \\
\hline$+400 \cdot \mathrm{Hz}$ & $587.06 \cdot \mathrm{cP}$ \\
\hline$-400 \cdot \mathrm{Hz}$ & $559.50 \cdot \mathrm{cP}$ \\
\hline$-300 \cdot \mathrm{Hz}$ & $539.62 \cdot \mathrm{cP}$ \\
\hline$-200 \cdot \mathrm{Hz}$ & $567.72 \cdot \mathrm{cP}$ \\
\hline$-100 \cdot \mathrm{Hz}$ & $706.19 \cdot \mathrm{cP}$ \\
\hline$-50 \cdot \mathrm{Hz}$ & $976.84 \cdot \mathrm{cP}$ \\
\hline
\end{tabular}

\begin{tabular}{|l|c|c|l|}
\hline RHEOLOGICAL CLASSIFICATION & \multicolumn{3}{|c|}{ YIELD POWER LAN - PSEUDOPLASTIC } \\
\hline INITIAL YIELD STRESS & $T$ & 46.7134 & Pascals \\
\hline CONSISTENCY FACTOR & $K$ & 18.3769 & Pascal-Sec \\
\hline NON-NEWTONIAN FLON FACTOR & $n$ & 0.3877 & DimensionIess \\
\hline CURVE FIT ACCURACY & $R^{2}$ & 92.8660 & Percent $(\%)$ \\
\hline
\end{tabular}


ENEFGY \&. ENUIFONMENTAL FESEAFICH CENTEFI COAL EENEFICIATION FROJECT

Grand Forks, North Dakata 58202

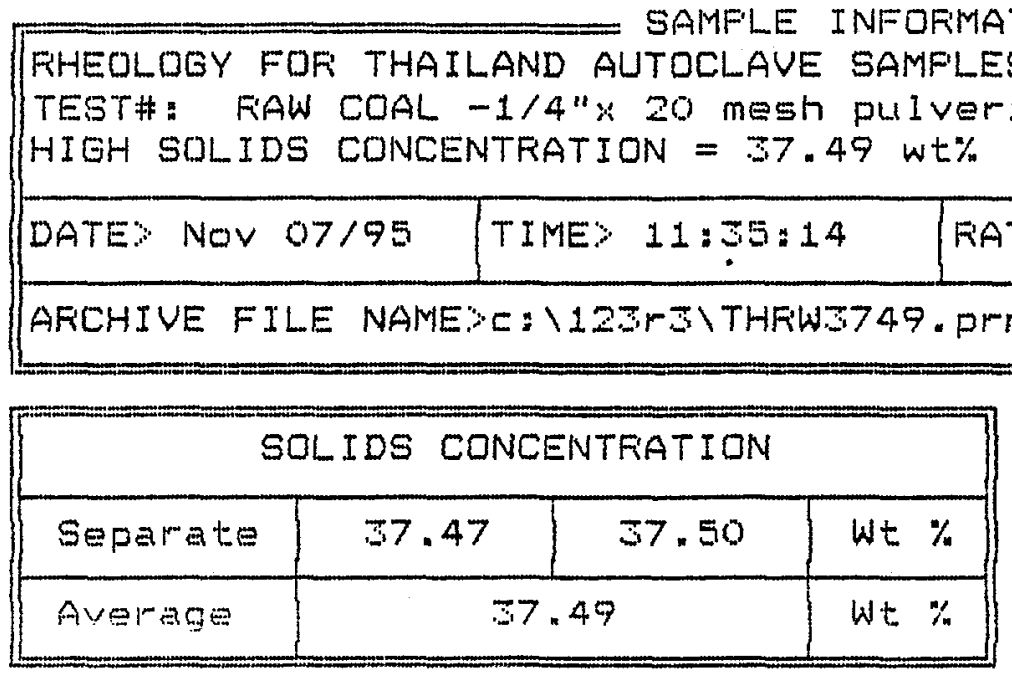

\begin{tabular}{|c|c|c|c|}
\hline \multicolumn{4}{|c|}{ ASH CONCENTRATION } \\
\hline$S \Leftrightarrow p a r a t$ & 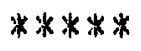 & $* * * * *$ & Wt $m / n$ \\
\hline Average & \multicolumn{2}{|c|}{$* * * 4 *$} & wt $\%$ \\
\hline
\end{tabular}

\begin{tabular}{|c|c|c|}
\hline \multicolumn{3}{|c|}{ VISCONETER CONFIGURATION } \\
\hline Sensor & $M V-I I F$ & Sensor Type \\
\hline 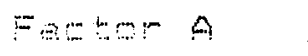 & 5.76 & Fascals \\
\hline$m a c t u m$ & $4 \times 40$ & $1 / \sec$ \\
\hline Temperature & 25.00 & ○Celcius \\
\hline$\% D$ & 100.00 & Percent (\%) \\
\hline$\%$ Tau & 70.00 & Fercent $(\%)$ \\
\hline
\end{tabular}

\begin{tabular}{|c|c|}
\hline $\begin{array}{l}\text { SHEAR } \\
\text { RATE }\end{array}$ & $\begin{array}{l}\text { AFPARENT } \\
\text { VISCOSITY }\end{array}$ \\
\hline$+50 \cdot H=$ & $1186.5 \overline{C F}$ \\
\hline$+100 \cdot \mathrm{Hz}$ & $85.8 \cdot 87 \cdot c F$ \\
\hline$+200 \cdot H=$ & $638.58 \cdot \mathrm{cF}$ \\
\hline$+300 \cdot \mathrm{Hz}$ & $536.15 \cdot \mathrm{CF}$ \\
\hline$+400 \cdot \mathrm{Hz}$ & $444,74 \cdot C F$ \\
\hline$-400 \cdot 1-12$ & $416.77 \cdot C P$ \\
\hline$-300 \cdot H=$ & $402.05 \cdot C P$ \\
\hline$-200 \cdot \mathrm{Hz}$ & $4.28 \times 45 \cdot C F$ \\
\hline$-100 \cdot \mathrm{Hz}$ & $536 \cdot 55 \cdot c P$ \\
\hline$-50 \cdot 1=$ & $749.18 * C P$ \\
\hline
\end{tabular}

\begin{tabular}{|c|c|c|c|}
\hline FHHECILOGICAL CLASSIFICATION & FSEUD & STIC & \\
\hline INITIAL YIELD STRESS & $\tau$ & 0.0000 & Pascals \\
\hline CONSISTENCY FACTOR & k: & 5.2853 & Fascal.Secn \\
\hline NON-NEWTONIAN FLOW FACTOF' & $n$ & 0.5975 & Dimensionless \\
\hline CURVE FIT ACCURACY & $F^{2}$ & 99.1077 & Percent $(\%)$ \\
\hline
\end{tabular}


ENERGY \& ENUIRIONMENTAL FESEAFCH CENTER

COAL BENEFICIATION FFOJECT

Grand Forks, North Dakota 58202

\begin{tabular}{|c|c|c|}
\hline \multicolumn{3}{|c|}{ 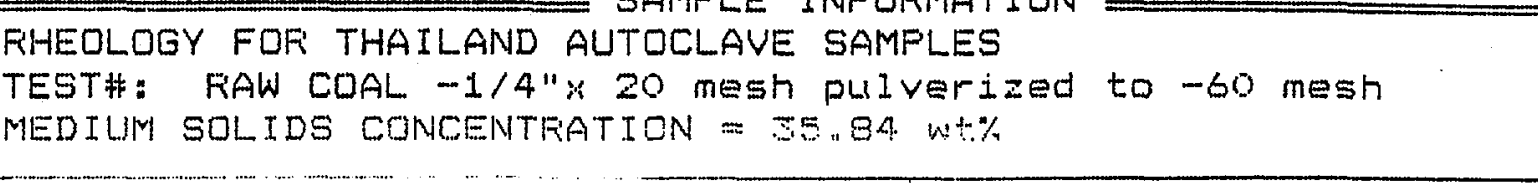 } \\
\hline DATES NOV $07 / 95$ & TIMES 11:47::1 & FATES 120 samples/minute \\
\hline
\end{tabular}

\begin{tabular}{|c|c|c|c|}
\hline \multicolumn{4}{|c|}{ SOLIDS CONCENTRATION } \\
\hline 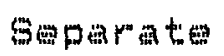 & 3.85 & 35.85 & wt $\%$ \\
\hline Average & \multicolumn{2}{|c|}{35.84} & Wt $\%$ \\
\hline
\end{tabular}

\begin{tabular}{|l|c|c|c|}
\hline \multicolumn{3}{|c|}{ ASH CONCENTRATION } \\
\hline Separate & $* * * * *$ & $* * * * *$ & wt $\%$ \\
\hline Average & ***** & Wt $\%$ \\
\hline
\end{tabular}

\begin{tabular}{|l|c|l|}
\hline \multicolumn{2}{|c|}{ VISCOMETEF CONF IGUFATIDN } \\
\hline Gensor & MU-IIF & Sensor TYPE \\
\hline Factor A & 5.76 & Pascals \\
\hline Factor M & 4.40 & $1 /$ sec \\
\hline Temperature & 25.00 & OCelcius \\
\hline$\%$ D & 100.00 & Percent (\%) \\
\hline$\%$ Tau & 40.00 & Percent (\%) \\
\hline
\end{tabular}

\begin{tabular}{|c|c|}
\hline $\begin{array}{l}\text { SHEAF' } \\
\text { RATE }\end{array}$ & $\begin{array}{l}\text { AFPAFENT } \\
\text { VISCOSITY }\end{array}$ \\
\hline$+50 \cdot \mathrm{Hz}$ & $389.45 \cdot C F$ \\
\hline$+100 \cdot H z$ & $295.44 \cdot \mathrm{CP}$ \\
\hline$+200 \cdot \mathrm{Hz}$ & $25.89 \cdot \mathrm{CP}$ \\
\hline$+300 \cdot 12$ & $216.68 \cdot c F$ \\
\hline$+400 * \mathrm{~Hz}$ & $197.55 \cdot \mathrm{CF}$ \\
\hline$-400 \cdot H z$ & $195.88 \cdot \mathrm{cP}$ \\
\hline$-300 \cdot H z$ & $192.96 . \mathrm{CF}$ \\
\hline$-200 \cdot H z$ & $210.61 . \mathrm{CF}$ \\
\hline$-100 \cdot H z$ & $265.86 \cdot c \%$ \\
\hline$-\quad 50 \cdot \mathrm{Hz}$ & $379.49 \cdot \mathrm{CP}$ \\
\hline
\end{tabular}

\begin{tabular}{|c|c|c|c|}
\hline RHEOLOGICAL CLASSIFICATION & PSEUD & STIC & \\
\hline INITIAL YIELD STRESS & $\tau$ & 0.1198 & Pascal: \\
\hline CONSISTENCY FACTOR & $k$ & 1.3395 & Fasca $1 \cdot \sec n$ \\
\hline NON-NEWTONIAN FLDW FACTOR & $n$ & 0.6771 & Dimension Iess \\
\hline CUFIVE FIT ACCURACY & $F^{2}$ & 99.8367 & Percent $(\%)$ \\
\hline
\end{tabular}


ENERGY \& ENUIFONMENTAL FESEAFICH CENTEF:

COAL EENEF ICIATION FFIOJECT

Grand Forks, North Dakota 58202

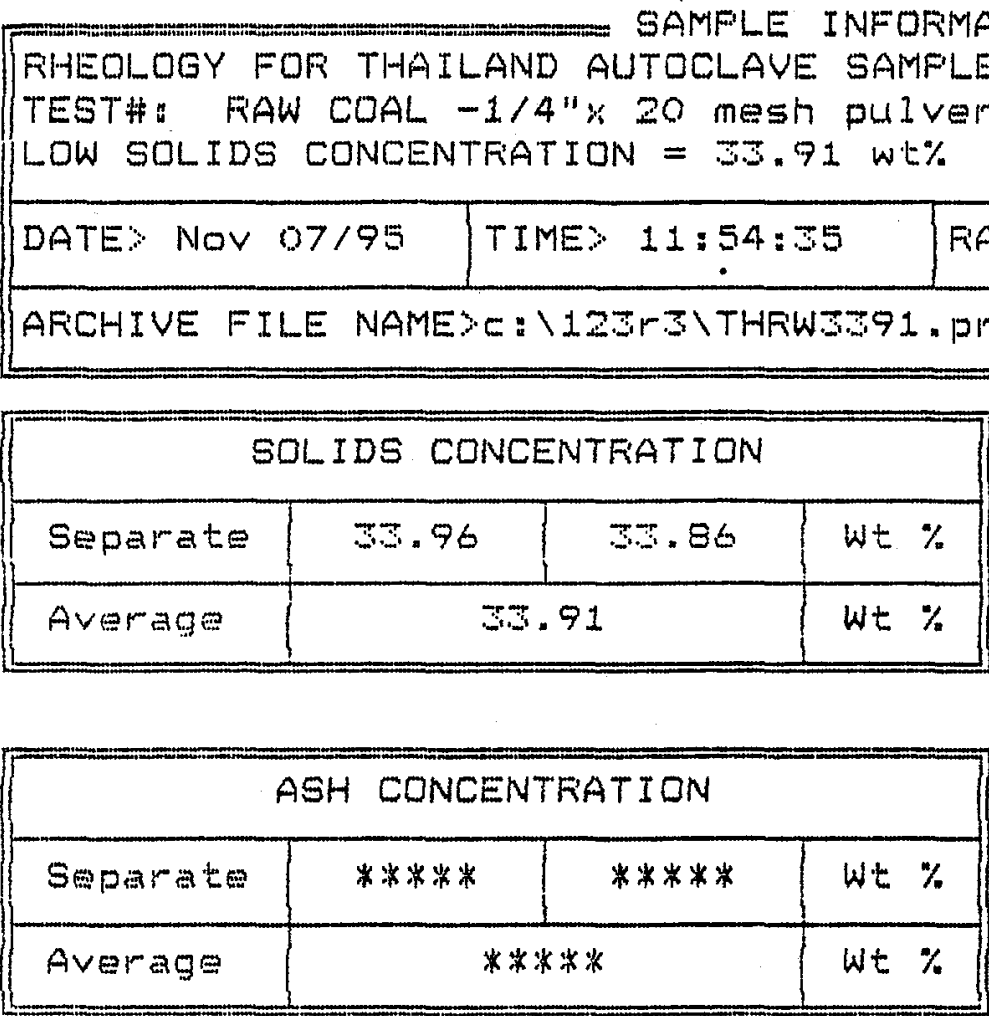

\begin{tabular}{|c|c|c|}
\hline \multicolumn{3}{|c|}{ VISCOMETEF CONFIGURATION } \\
\hline $5690 \%$ & $M Y-I I F$ & Sensor TYPe \\
\hline Factor A & \pm 76 & FascaIs \\
\hline$=a c t=m$ & 4.40 & $1 / \sec$ \\
\hline Temperature & 25.00 & - Celcius \\
\hline$\% D$ & 100.00 & Fercent $(\%)$ \\
\hline$\%$ T政 & 20.00 & Fercent $(\%)$ \\
\hline
\end{tabular}

\begin{tabular}{|c|c|}
\hline $\begin{array}{l}\text { SHEAF } \\
\text { RATE }\end{array}$ & $\begin{array}{l}\text { APFAFENT } \\
\text { VISCOSITY }\end{array}$ \\
\hline$+50 \cdot 4 z$ & $150.54 \cdot C F$ \\
\hline$+100 \cdot \mathrm{Hz}$ & $113.94 \cdot \mathrm{CP}$ \\
\hline$+200 \cdot H z$ & $89.29 \cdot \mathrm{CP}$ \\
\hline$+300 \cdot H z$ & 8I.14.CF \\
\hline$+400 * \mathrm{~Hz}$ & $78.73 \cdot \mathrm{EP}$ \\
\hline$-400 \cdot H z$ & $80.52 * C F$ \\
\hline$-300 \cdot H z$ & $83.93 \cdot C F$ \\
\hline$-200 \cdot 4 z$ & $72.59 \cdot \mathrm{cP}$ \\
\hline$-100 \cdot 4 \mathrm{H}$ & $117.45 \cdot \mathrm{CP}$ \\
\hline$-\quad 50+1+2$ & $167.85 * \mathrm{cF}$ \\
\hline
\end{tabular}

\begin{tabular}{|c|c|c|c|}
\hline FHEOLOGICAL CLASSIFICATION & \multicolumn{3}{|c|}{ PSEUDOPLASTIC } \\
\hline INITIAL YIELD STREGS & $T$ & 0.7698 & Fasca \\
\hline COIVSISTENCY FACTOR & k: & 0.4 .31 & Fascal Secr \\
\hline NON-NEWTOIVIAN FLOW FACTOR & $n$ & 0.70 .28 & Dinension $1 \in 5$ \\
\hline CURVE FIT ACCURACY & $F^{2}$ & 99.7468 & Fercent. $(\%)$ \\
\hline
\end{tabular}


Energy \&

Environmental

Research

Center 
Appendix D

Date: 11/29/95

Process Development Unit (PDU) \#74

Feed Material: Thailand lignite slurry

Particle Size: -60 mesh

Feed slurry flowrate (lb/hr): 525

\section{Conditions}

temperature (degrees F): 575

set condition pressure (psi): $2100 \mathrm{psi}$

est. residence time (min): 7 minutes

Length of test (min): 105 


\begin{tabular}{|c|c|}
\hline$E 1$ & SIurry Fre-Henter ir Iet \\
\hline Ei & 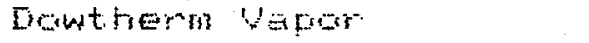 \\
\hline & Dowtherril i_iglad \\
\hline & at:er Coil Irilet \\
\hline & Fretreater coil out let \\
\hline
\end{tabular}

\begin{tabular}{|c|c|}
\hline$E$ & Slumry Fre-Heater Irilet \\
\hline & Dowitherm Yapor \\
\hline & Dowthern Liquid \\
\hline & Fratisater Coil Irilet \\
\hline & Fineheater Coil Outlet \\
\hline & Su urry Fre-Heater Irilet \\
\hline & 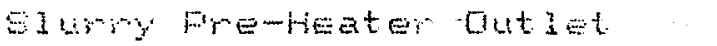 \\
\hline & Mowrititerro Liquid Temperature \\
\hline & 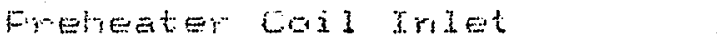 \\
\hline & 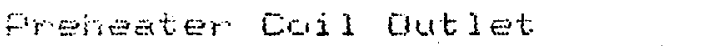 \\
\hline
\end{tabular}

Esh Frebeater $\rightarrow$ Reactor Trangfer Line

\begin{tabular}{|c|c|}
\hline$P i$ & Reactor dutlet \\
\hline BI & 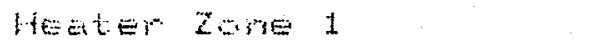 \\
\hline$\dot{i}$ & HEREr Irre \\
\hline$\dot{i}$ & Heoter $z<r=z$ \\
\hline 3 & 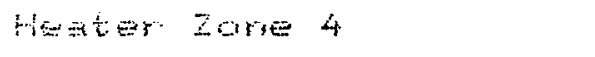 \\
\hline & Bescotont $1 \mathrm{E}$ \\
\hline & 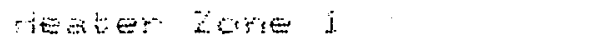 \\
\hline & 10 \\
\hline - & 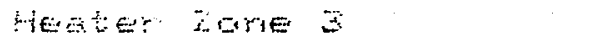 \\
\hline$\cdots$ & 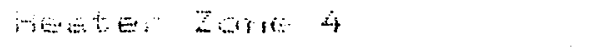 \\
\hline & seperatminguid \\
\hline & bepertot Vessel Liquid \\
\hline & 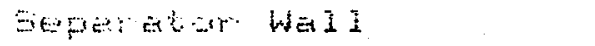 \\
\hline & $\theta \pm$ wa \\
\hline Q in & 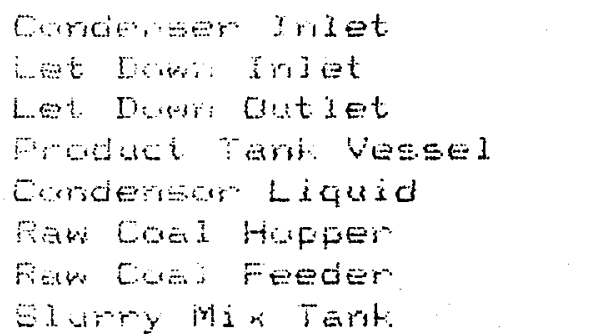 \\
\hline
\end{tabular}

EETYFrES. OLIT

EE-D'ETH. QUT:

EE-DLGTH. OLTE

EE-CI Ir. OUTE

EE-CIOut. aure

EE-ESFTE. OUT

ES-DVFTr. OUTE

EE-DLGTH. OUTE

EE-CI Ir. OUTE

E5-CIOut. OUTE

EEI-E9FrS. QLIT

E9OLFHES. QUT

E9-DLATri. OLTE

EG-CI I\%. OUTE

E9-CIOut. OUTE

E9-R3 XF. OUTE

FJ-Ot Tmp. OUTE RS-Zare1. OUTE FЗ゙-ZareE OUT2 R3-ZAME3. OUTE R3-Zare4. QUTE

PEOT.TMF. OUT

FE-zorie 1. OUT

RE-ZOHEE. QUT

FE-Tane3. QUTE

RE-ZGMEA. OUTE

51-Liqd. OLITE

$31-L=v E:$. OUT

91-wa I . outre

SE-LE: I. OUT

RE-zoreE. OLTE:

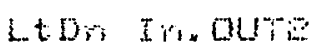

Ltbir aut cuts=

FroTHLV . OLT

Cro-LEYI. OLIT

HopkEr. OUT

Feeder. OUT

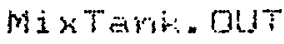

1820.95

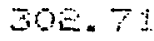

드. 5

E⿺辶一由

$3=1.42$

1742.

479.60

499.07

उEㅌㄹ. 71

4 E. 91

2095.47

$=103.43$

608. 78

4 든. 90

574.30

576.31

597.06

들.79

575.10

591.51

596.75

$=7.81$

$53.2=$

EE: 11

$13 E .35$

134.15

1凷。证

50.59

$1 E=58$

르.4 4

I3i.

: 50,6

$=39.35$

0. 00

39.44

0.0

170. 59

40.05 
Slurry fre-Heater Irilet

Ei

EI

$E I$

EI

$E$

$E Z$

$E$

$E$

Ee

$E 3$

$E 3$

$E S$

E.3

$E 3$

EaR1 freneater $\rightarrow$ feector Trarsfer Lire

Ri Reactor out let

Fi. Heater Zone i

RI Heater zore

Fi Heater zore 3

Fi Heeter zorre 4

Pe Fentror Out iet

Fe Heater Zane i

Re hester zore

He Hester zare 3

ai idener zerse 4

B. Sefermtar Liquic

a beprato verol Liquid

51. Separator Wall

oE Seforetor Vesel Liquid

DOWD Curderser Iriet

inet Dowist Irslet

Let Down art iet

rubtict Tark vestel

Corrdersom Liquid

Derb Coa i Hopper

Serin Gaid Feeder

Simra mix Tart
EDI rFires. DUT

EE-DVPTH. QUTE

EE-DLQTM. DUTE

EE-C: IY. QUTE

EE-CIOUt. OUTE:

EE-ESPrS. DUT

ES-DVPTH. RUTE

ES-DLGTm. OUTE

EE-CI Ir. QUTE

ES-CIOut. OUTE

ES-EGPrs. DUT

E90tFres. OUT

E9-DLGTM. OUTE

E9-CI I H. OUTE

E9-CIOut. OUTE

EG-RE XF. QUTE

R3-0t Tmp. QUTE

R3-Zare 1. QUTE

R.3-Zarie:. QUTE

RZ-ZGriez. OUTE

R3-Zarie4. QUTE

FEQT TIMF. OUT

RE-Zare 1. OUT

RE-ZareE. QUT

RE-Zares. OUTE

PE-Zore 4 - OUTE

Si-Liqd. OUTE

SI-LEVE1. OUT

E1-Wa11. OUTE

SE-Level. QUT

Re-zoreE. oure

LtDri sri. ouTe

LtDr aut. GuTe

FraThLv1. OUT

Crid-Lev1. OUT

Happer. OUT

Feeder. OUT

MixTarit. OUT i 945.44

304.81

504.31

EES. $1 E$

345.00

1765.10

491.50

500.16

348.93

451.95

ㄴ1. 79

E12E. 31

604.44

$454.3 E$

$57 \equiv .04$

574.80

588.36

574.75

574.80

577.99

589.33

อ. $7 E$

53. 36

5 5. 63

134.15

134.90

175.76

57.56

133.91

EE. E

132.13

$564 \cdot 34$

2069. 41

6.00

42.43

0.00

179.75

40.87 


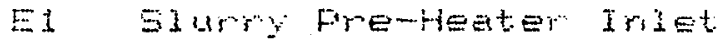

E1. Dowtherm Vapoi

E1. Dowther Liguic

El Freheater [oid Iriet

E1 Fretretien Coil Gutiet

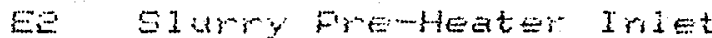

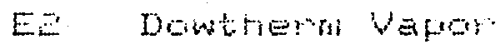

E: Dowthern Liquid

E: Frebeater Cail Tnlet

ED Frefleater Coil. Out Iet

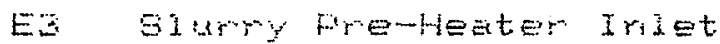

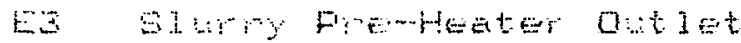

E3 Dumblara Liquid Temperature

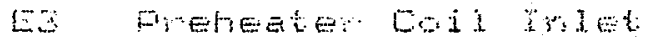

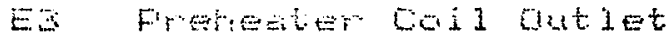

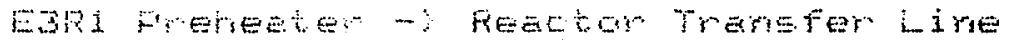

R1 Feacter Qut let

Ri Heater zome 1

P. l-ienter zorse

PI HeEter Zurs 3

W Hestar zare 4

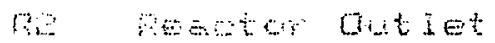

Pe Hester ante i

Pe Hester zome $=$

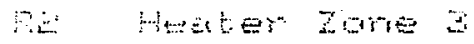

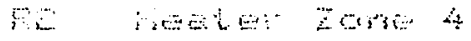

\begin{tabular}{|c|c|}
\hline $5:$ & SEparatar Liquid \\
\hline $9 i$ & Gerater VeseEI L.iquid \\
\hline 1 & 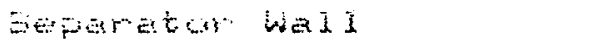 \\
\hline$\cdots$ & se-and or resend Liguid \\
\hline 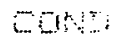 & Corder, \\
\hline & 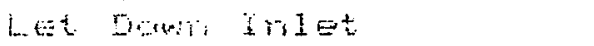 \\
\hline & Del Dant Gutlet \\
\hline & 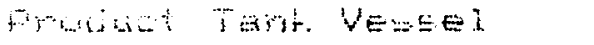 \\
\hline & 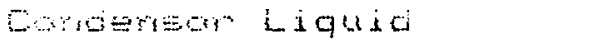 \\
\hline & 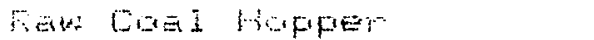 \\
\hline & 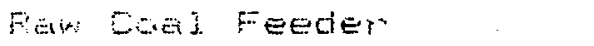 \\
\hline & Sikrry pith Terrt. \\
\hline
\end{tabular}

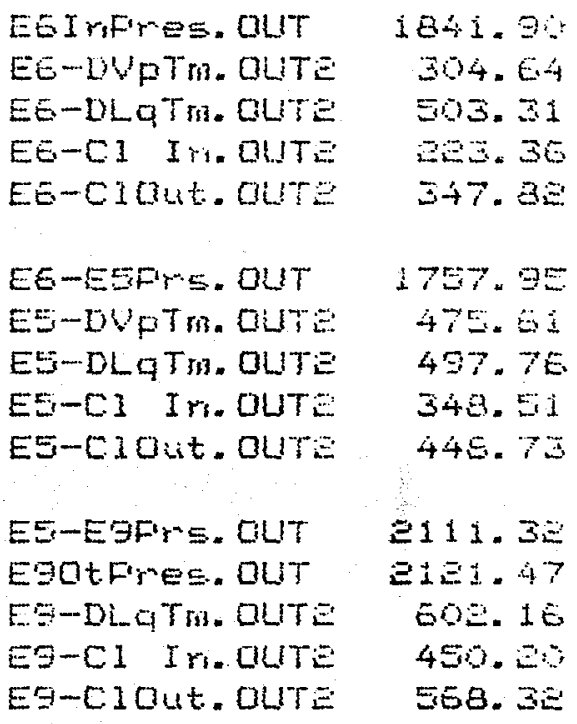

184i. $9:$

304.64

503. 31

$20-35$

$347.8=$

1757.95

$4 i \underline{G}$

$497.7 E$

349.51

445.73

ㄹ11. 32

로‥47

600.10

$450.9 \%$

568. $3=$

571.19

581.66

570.07

570.95

57. 5

$59=5$

31.35

53.00

5.5

$1+3.35$

133.6

199.

5.

142.65

프. 7

I 31.4 .6 357.44 a. 35

i) $\$ 0$

39.41

(3)

15.50

40.99 
E1 $\quad$ il arry wemleater IrIet

E1 Dowthern? Vapar

EI Dewtherm Liquici

E1 Fremedter Gii Tinet

Ei Pretreater Coi out let

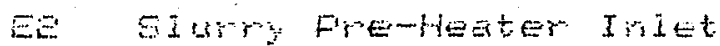

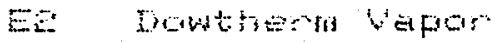

Ei Dowitherm Liquid

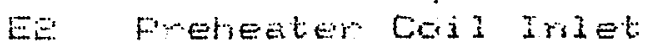

E: Frefleter Coil Dut let

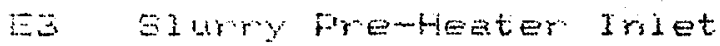

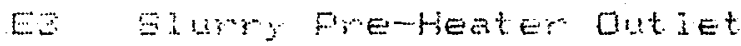

El Lumbrarn Liquid Tenperature

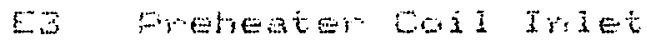

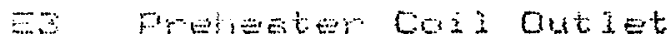

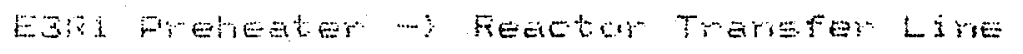

Fi. Featom out let

Ri hisater Zarse 1

Pl Hoater zane

Ri herat Zars $Z$

II bester zores it

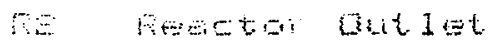

n. Hostore zore 1

O THeter Zure

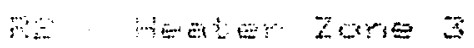

औin mate

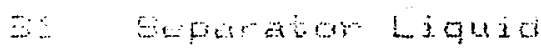

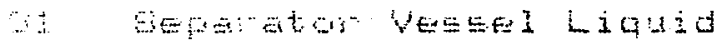

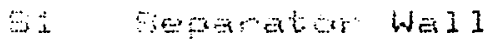

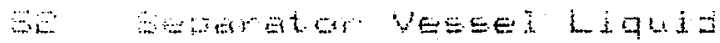

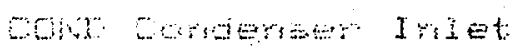

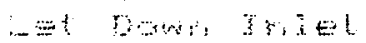

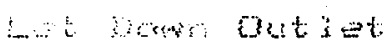

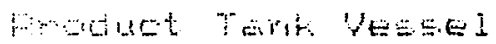

mostsers inaic

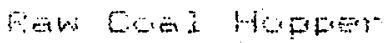

Hato Con a Feeder

andrty ma Tant
EEIRTRES. CUT

EE-DVPTH. CLITE

EE-DLOTH. OLTE

EE-CI Ir. QUTE

EE-CICHE. OUTE

EG-ESFrS. QUT

ES-DVpTn. OUTE

ESTLLTH. OLTE

ES-CI I H. QUTE

ES-CIOut. OUTE

EE-EGFrE. OUT

EgOtFres. DUT

ES-DLQTH. OUTE:

ES-C1 Ir. OUTE

E9-CIOut. OUTE

ES-RS XF. OUTE

PI-atTmp. QUTE

R3-Zane 1. OUTE

R3-ZCrEE. OUTE

R.Z-Zcries. OUTE

RE-20re4. OUTE

PEQt TMP. OUT

Pe-zorei. out

FE-zomeE. OUT

FE-Zanez. OUTE

BE-Zore4. Cun

S1-ijad. OUTE

S1-LeveI. OUT

S1-Waij. QuTE

se-Levei. out

Re-zoree oute

Ltim xin our

LtDn Gut. OUTE

FroTHEV . OLT

Crot WEVI. DULT

Hopper our

Feecier. OLT

mixterit. out
1950.05

307.70

505.26

230.74

343.15

1769.10

473.91

498,25

350.33

$44 E .5 E$

E123. 97

E1E. 20

004.51

450.20

571.25

573.39

583.17

571.92

57 E. 54

575.99

593.64

33.92

54.13

56.00

134.56

134.96

197. 55

53.90

152.57

2:. 40

135.8

घ6. 7

E53.60

0.00

43.24

0.60

160.55

40.91 
E1

EI

EI

E:

E.

$E$

$E$

$E$

E.

$E=$

E

23

sin

$E$

5

5

iุ

Pi

Fi

in

$\because i$

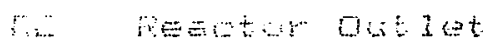

n Hetrotrobe i

ne Heatel $z$ arse

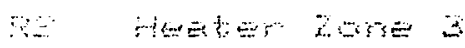

rit

$\because \vdots$
$\vdots$
$\vdots \vdots$
$\vdots$

gepertat -

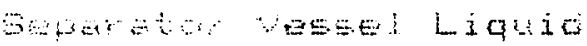

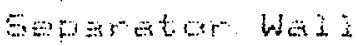

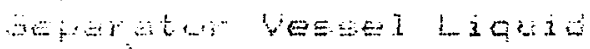

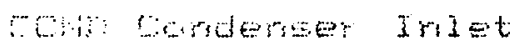

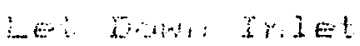

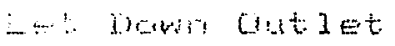

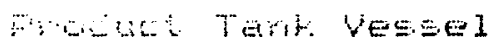

Emomos Liquid

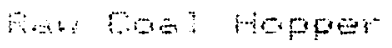

For Lued FeEcler

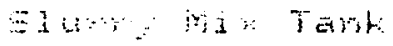

EEI PFTES. OUT

EE-DVFTH. OUT:-

EE-DLGTH. DLTT:

ED-C1 Ir. OUT:

EE-CDOut . CULT:

EE-ESFY. OUT

EE-IVWTH. OLT:

EE-DLGTM. OUT:

EE-CI Ir. OUIE

Es-C1OHt. OUT:

EE-EGFrS. OUT

Egotfres. Qur

ES-DLqTH. OUTE

E9-CI IN. QUTE

E9-CIOUt. OUTE

E9-R3 XF. QUTE

R3-0t Tmp. OUT:

RZ-ZGM 1. OUTE

PJ-ZGreE. OUTE

RS-ZGrE3. DUTE

Fே-Zarté OUT:

PEOt Trmp. DLLT

RE-zorse1. OUTT

FE-Z Z Z

f.

PE-ZOHE4. OUIT

$5 i-L i q d .04 T=$

Si-Levei. OU

5i-ba11. CUL"

SE-he EE. QU!

RE-ZET+E. OUT

LtDr, Ir. OUTE

Ltedr Out. OUTH:

"rotrit. OL"

Crid-l_ew . Culs

Hopper. out?

Feedzr. OUT

mi
$1697 . \%$

367.27

504. 5

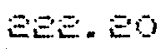

345.47

1010.34

476.75

495.95

34 E. स

443.09

리견.7E

루 71.07

$60=91$

445.10

든.69

570.56

$58=.66$

E71. 37

572.56

574.13

폰.77

34.96

53. 13

S논. 15

130.30

$\pm 3.5$

$=4+94$

70.6

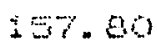

ㅍ․ 9

13.31

$560 \%$

者:

1) 30

$42=94$

2433.73

ज्ञान. 90

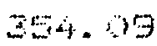


E2.

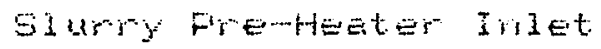

E1 Dowtherm Yerpar

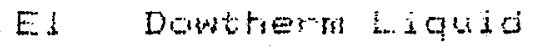

Ei Freheater Coil Irilet.

Ei Frefreater Eoil outiet

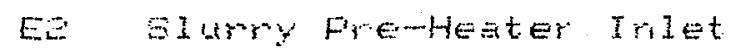

E.:

$E$

$E$

$E$

Dowthem verpor.

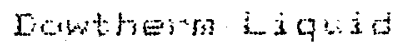

pretieater [oil indet

Freheater Cois out 1 é

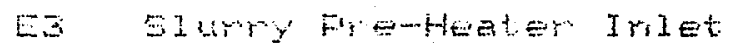

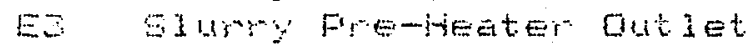

ES Fowriberri Liquid Temperature

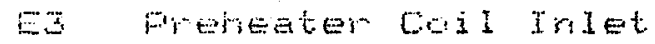

Ea Fretreater tail outiet.

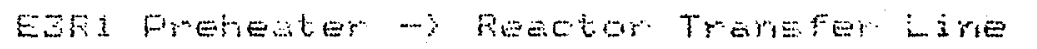

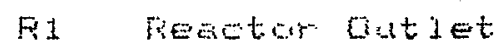

PI Heater atre 1

PI Heater Zarie

Pi Hevter Zare 3

Ri Horler 7 mre 4

Re Destor ot let

P. Hoter zerse 1

No lester Ionu

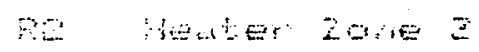

no biterter zose is

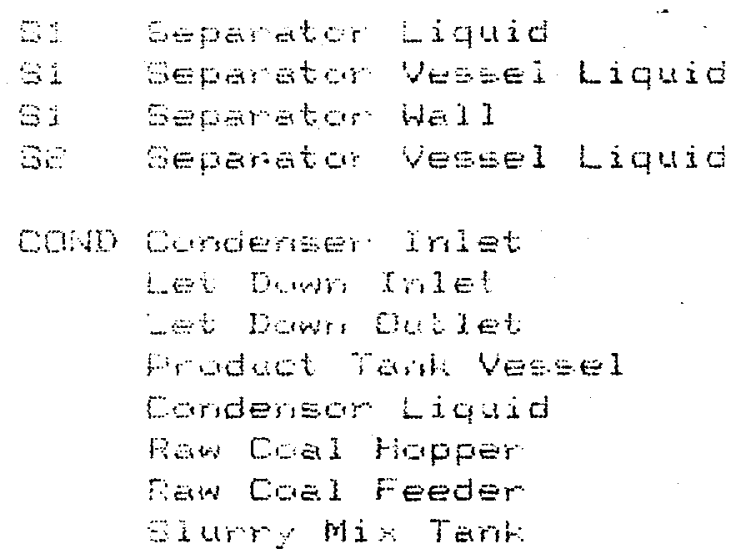

EEInFYrES. DUT

EE-DVpTm. OUTE

EE-DLqTr. OUTE

EE-CI Ir. DUTE

EE-CIOUT. OUTE

EE-ESFrE. OUT

EE-DVPTH. OUTE

EE-DLGTm. OUTE

ES-OI Ir. OUTE

ES-ClOUt. OUTE

EE-E9FrS. QUT

E90tFres. QUT

ES-DLGTm. OUTE

ES-C1 Ir. OUTE

EG-C10ut. OUTE

E9-R3 X. OUTE

R3-Ot Tmp. QUTE

R3-Zarre1. OUTE

R3-zoneE. OUTE

R3-ZarES. DUTE

R3-zarie4. DUTE

ReOt ThP. OUY

PE-ZEred. OUT

RE-Zunte. OUT

RE G GrES OUTE

RE-ZCSEA. OUTE

Sj-Liad. Oute

Si-Leve1. DUT

si-idal 1. OUTE

ge-ievei. UUT

RE-ZTrEE. OUTE

Ltom ir. oute

LtDrs dut. DUTE

FraTKLVI. DU?

Croj-LeV3. OUT

Hopper. OUT

FeEder. OUT

MisTarin. OUT
1785.60

300.33

504.90

20.51

31.79

173. 50

473.2

497.97

317.18

451.28

2080.94

0084.60

215. 00

451.60

589.65

590.10

579.91

579.71

569.59

574.99

581.84

35.93

프. 25

5.3 .99

120.63

$13=16$

207. 21

5.99

15. 5.9

ㄹ. 37

129.13

500.91

205. 95

0.0

40.89

95.73

200.00

19:-E: 


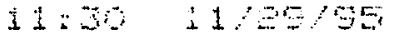

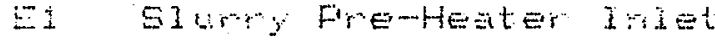

E1 Dotrtherm Vafors

El Dowthein Liquicio

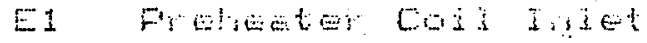

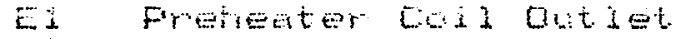

\begin{tabular}{|c|c|}
\hline$E$ & SIMry pre-Hester IMIEt \\
\hline$E=$ & Dowtinerra Yapar \\
\hline$E=$ & Dowitherrt Liguis \\
\hline $5=$ & Frathenter Coil Irilet \\
\hline$E \Xi$ & Frebedter Coij Obtat \\
\hline$E$ & $P,-H \leq a t \in r^{2}$ \\
\hline $6=3$ & 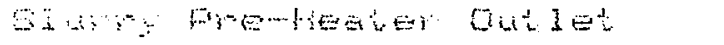 \\
\hline$[-1-1$ & 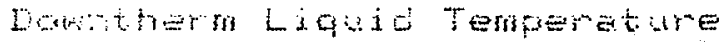 \\
\hline 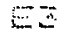 & 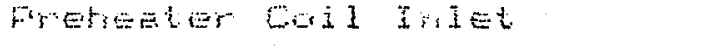 \\
\hline 4 & 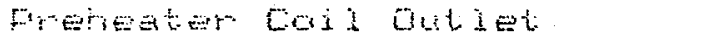 \\
\hline
\end{tabular}

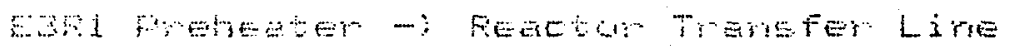

Pi Rearon Oitiet

PI HEater ZAOE 1

P1 Henter Zare

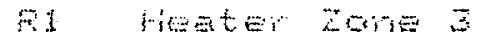

ni menter zare 4

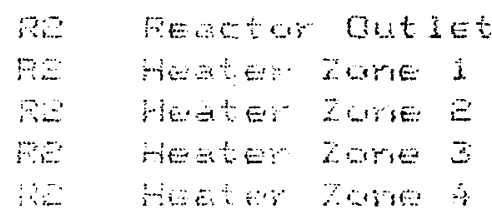

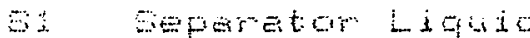

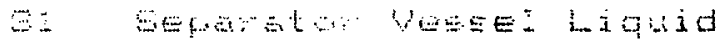

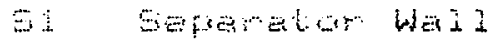

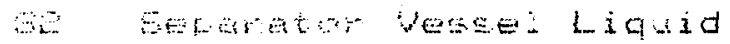

wow bondertor $1 \mathrm{~m}$ ist

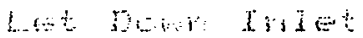

i...t. Yow

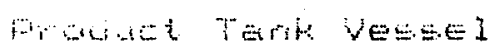

Cudoryor Liquici

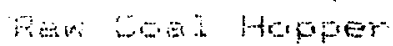

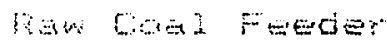

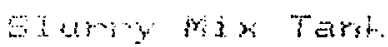

EEI YFFES. OLT

EE-DVpin. OUT:

EE-DLGTH. OUITE

EG-C3 In. OLTL

EE-CIQHt. QUTTE

EE-ESFrE. QUT

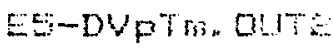

E5-DLG Th. OUT:

EE-CI J\%. QUTE

EE-CIOUt. OUT:

EET-EGF' $r=$ QLT

E90tFres. OUT

Eg-DLGTH. OUITE

ES-C.I I r. OUTE

Eg-CiOut. OLTE

ES-RS XF. OUTE

R3-Ot THP. OUTE

RA-Zore 1. OUTE

Ris-zoreE. OUTE

P3-ZGHES. OUTE

RE-ZGrEA. OUTE

REOR ThP. OLT

RE-zore . OLIT

RE-ZOMER. OUT

7E-Zores. QLTE

RE-2.

51-Ligd. OLTE.

S? - LE:

$51-4$ a 13 . 041

BE-LEYEI. OUT

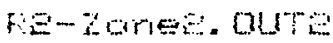

LAOH Th. OU?

tobr, का: . OUT:

Frethis. alt

Crot-Leri. 0u:

HEpper. OU:

Feeder. aut

Mixirarito OLI
1973. iE94.

500.04

근. 46

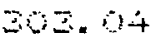

1000.0

454,20

$49=70$

304.6

$4=4.81$

E143. 47

E\$4 44

Eis. 73

A EE. I

569. 13

$57 a+10$

57E.

571.71

573.71

574.69

59: $=$

36.19

ㅍ. j.

5.19

1.

129.9

E19.6s

15. 36

IEE. =

E. 45

47.81

565,23

$2-7$.

1.

20.5

840.5

अ00. 00

170.79 
Appendix D

Date: 11/29/95

Process Development Unit (PDU) \#74

Feed Material: Thailand lignite slurry

Particle Size: -60 mesh

Feed slurry flowrate (lb/hr): 525

\section{Conditions}

temperature (degrees F): 615

set condition pressure (psi): 2100 psi

est. residence time (min): 7 minutes

Length of test (min): 90 
FDL Fraces: Data

$104010 \quad 11 \% 969$

E1 S1 urry Prempleater Iriet

EEIrFreE. OUT

EE-DUFin. OLITE

EE-DLGTH. CUTE

EE-CI Im. OUTE

EE-CiGut. CUTH

EE-ESFIS. OUT

EE-TViTin. OUT:

EE-DLGTH. OLTE

ES-CI In. OUTE:

ES-CIOut. OLIS

EE-E9FT=. OLT

E90tFTE. OUT

ES-DLATM. OUTE

E9-CI Ir. OUT:

E9-CI QUE. OUTE

E9-R3 Xf. OUTE

R3-Ot TIR. OUTE

R3-zare1. OUT:

RS-ZGreE. OUTE

RS-Z̈YEZ. OLTE

PJ-ZGreA. CULTE

Rate Tho. OUT

PQMUITE1. OUT

RE-ZIFIE. QULT

Fi-zures. CUT:

Re-zome4. DLP

51-Liqd, oure

SI-LEWE1. OUT

51-WE1 1. GLITE

SE-LEVE1 . OUT

Bu-zarté, QUTE

LeDr, Tr. QUTE

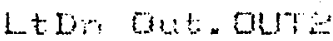

FrdThL1. GUY

Crid-Levi. OLT

HOPFE . OUT

Feeder. OUT

Min inth OUT
3949.30 330,43

506. 54

를. 41

364.45

i $7 B=2$. 평. 85

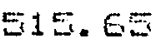

564. 4

$47 \%$.

2114.39

119.63

E54.94

$477.3 \pm$

600.04

501.75

61‥17

이.06

E01. 35

605. 5

EI4. IE

36.44

$50 . \in 9$

$5=.54$

$1=7.60$

$10.9=$

203.29

59.39

i 10.30

E2.44

IEE. $\%$

Ë‥

ETi. 3

1. बो

4 . $E 6$

E引

Eण) 0री

383.97 
Ei Siurry Fremeater Injet

E1 Dowtrerm Vapar

Ei Dowthern Liquid

E. Freheater Coil inlet

E1 Freheater Coril outlet

\begin{tabular}{|c|c|}
\hline$E E$ & $\begin{array}{l}\text { Slury Fre-Heater Inlet } \\
\text { Dowthern vepor }\end{array}$ \\
\hline & Dowtherm Liquid \\
\hline & Frenteater Coil Inlet \\
\hline & Frelieater Coil Dutiet. \\
\hline & 5lurry tre-Hedter Irlet \\
\hline & Siurry Fre-Heater 0ut let \\
\hline & Downtherm Liquid Temperature \\
\hline & Freneater Cois InIet \\
\hline & Wreneater coil out let \\
\hline
\end{tabular}

Eani prebeater - Reactor Trenefer Lire

\begin{tabular}{|c|c|}
\hline $\mathrm{RI}$ & Reestor Qutiet \\
\hline $8 \dot{1}$ & Hester Zome 1 \\
\hline Pi & Heater Zurse $\mathrm{Z}$ \\
\hline $\mathrm{ns}$ & Menter Zure 3 \\
\hline तi & Heater zarse 4 \\
\hline$n=$ & $F=a n$ out 1 et \\
\hline ar & Heater 2are 1 \\
\hline$n$ & Henter zorme \\
\hline$F$ & Henter zarte 3 \\
\hline$\therefore$ & 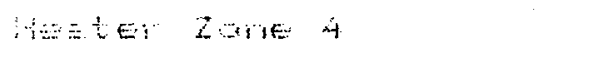 \\
\hline 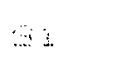 & jepentor Liquid \\
\hline$\because$ & 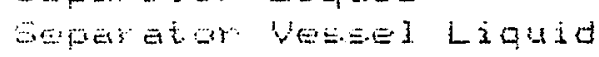 \\
\hline 5 & Wepartar bali \\
\hline$\sigma E$ & 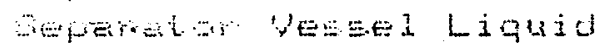 \\
\hline $\operatorname{ron} 1$ & Quriteren Indel \\
\hline & 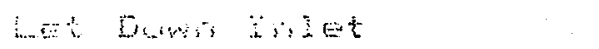 \\
\hline & 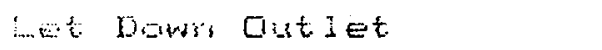 \\
\hline & Wublot Tril Vessei \\
\hline & Curves:orr Liquid \\
\hline & Str coa Hopper \\
\hline & Rew Ecas Feeder \\
\hline & Stimy lint Tarit: \\
\hline
\end{tabular}

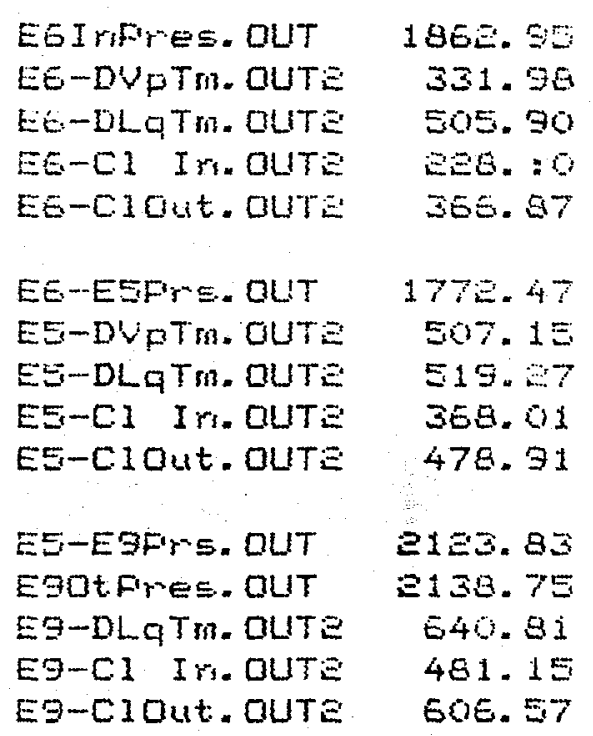

$19 E E . S B$ 331.95

505.90 ees.:0 365.97

1770.47 507.15 E19. .7 368.01 478.91

21ㄹ. 83

1ㅛ.75

540.81

481.15

EOE. 57

607.27

619.83

605.16

609. 61

613.79

$6=1.79$

35.60 50. 18

모. 19

1 20.69

$1 \mathrm{eg} .04$

อง9. 5

E1. 91

171.19

EE. 41

1.5. 95

5อง. 17

274.14

0.00

40.38

ece. 69

200.00

$303 .=1$ 


\section{1.: 30 11:20:90}

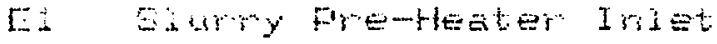

E1 Dowtherro Vapor

Ei Dowtherm Liquid

EI Fuentater Coi l r bet

Ej Wretret

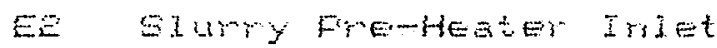

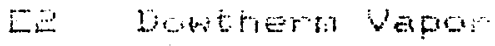

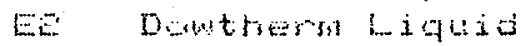

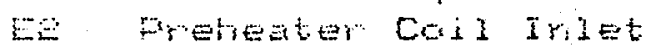

EE Frereater Cuis Qutiet

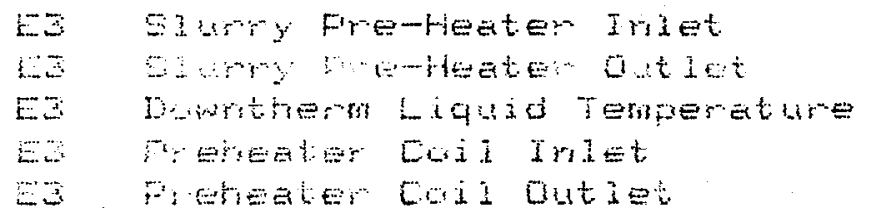

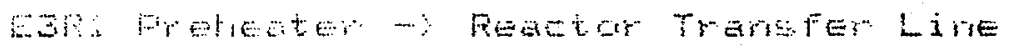

Pi PEt

Ri heater aris 1 .

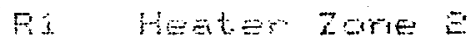

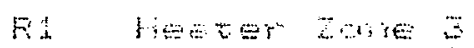

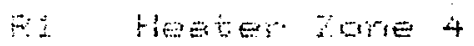

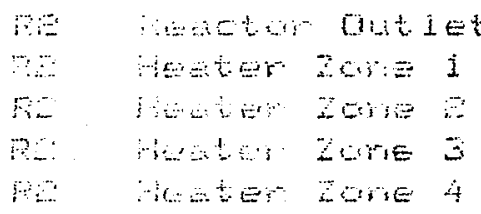

is bejatar liaujo

oi $\quad$ in

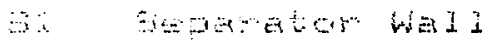

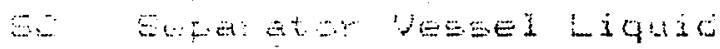

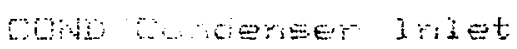

tot

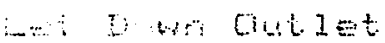

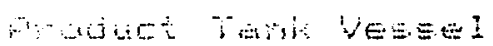

Wurberear inquja

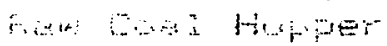

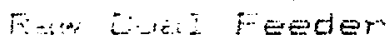

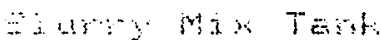

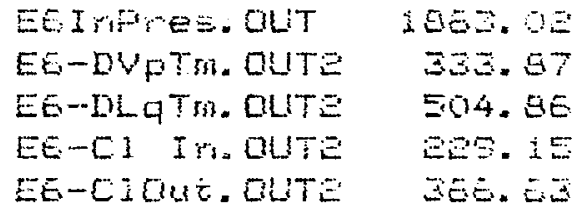

EE-ESFY . OUT 1770.70

EE-DVETR. OUTE $=10.53$

EE-DLqTn. OUT: - SE:-63

EE-EI In. UUTO $36 \%$.

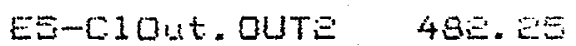

ES-EGFH. QUT $21 E^{5} .71$

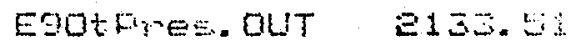

E9-DLGTM. OUTE . 44.99

E9-CI Ir. OUTE 404.30

Es-LIOUt. OLTE 609.45

E9-RE XF. OUTE 610.50

R3-OtTmp. OUTE E24. E5

R3-Zare1. OUTE 609.75

RJ-ZoreE. OUTE $\quad 613.33$

RI-ZCHEJ. OUTE ESG. $=9$

RZ-Zore4. OUTE GEE. EO

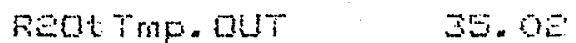

FE-Zare1. OUT 49.30

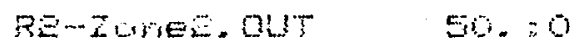

FE-zamej. QULTE IES. 10

Fin

5i-Liqd. OUT: ت\$1. EE

S.-LEVE. OUT GI.

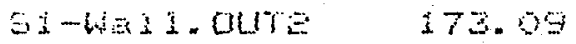

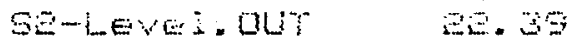

RE-ZGYEE. QUTE IES.

Ltom In. OU?

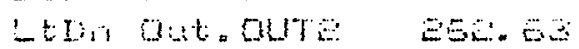

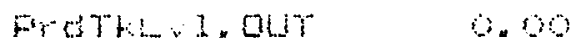

Crod-LEWI. CIST 41. 39

Hopper. OUT EE1E.55

Feeder. CLIT ZOC. OC

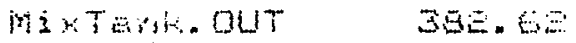




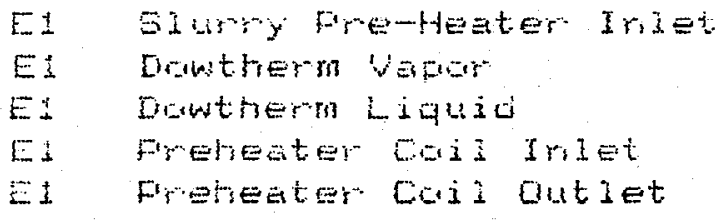

Ee Simry Fre-Heater Iriet

ED Domtinem vapor

Ee Dowtherm Liquid

EE Fretecter Coil Irilet

Ee Freheater Coil Outilet

E3 Siwry Fre-Heater Iriet

ES Siurry pro-heater out iet

Ea Downthera Liquid Terpereture

Ea metater Coil Inlet

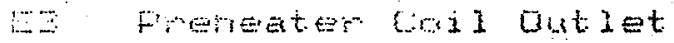

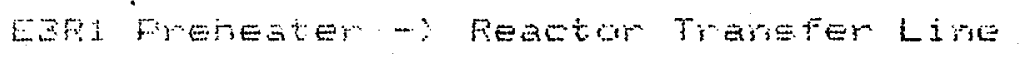

Pi Peactir outiet.

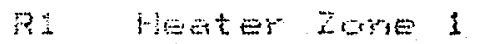

QI Healis Zare

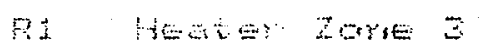

Di He tite zore i

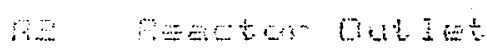

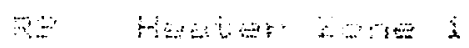

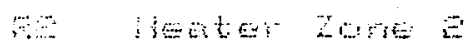

Fo Hester Zare

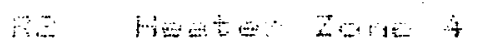

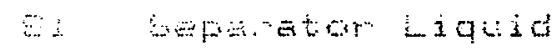

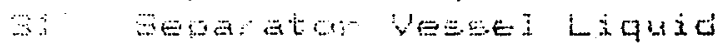

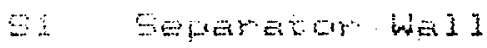

- ... -

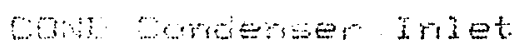

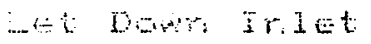

-

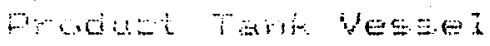

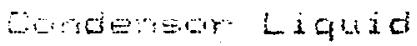

Fan Gerel lispper

Sew Conal Feeder

Difry rim Tarit.

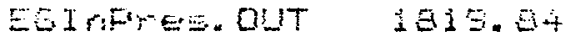

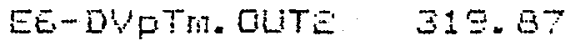

EE-DLATH. OUTE 503.79

EE-CI IN. OUTE 175.52

EE-CIOLE. OUTE 3EZ.79

EE-ESFrS. OUT

17E1.45

ES-DUFTH. OUTE

ES-DLGTM. CUTE

EE-CI ITI. OUTE

EE-CIOUt. QUTE

EE-ESFrs. QUT

EoOtFres. OUT

Eg-DLqTH. OUTE

500.57

516.75

$3=4.95$

450.01

2072.39

2003.30

643.79

457.70

ES-CI Ir. OUTE

E9-CIDut. OUTE

598.10

EG-RS XF. OUTE

$000.3=$

RS-OtTMP. QUTE

R3-ZGNE 1. QUT:

P3-Zorez. OUTE

RS-Zares. OUTE

p3-zare4. OUTE

E13. 27

596.50

601.47

60.11

$619.4 a$

ReOt THF. QUT

PE-zorsej. OUT

RE-Z MiseE. OUT

RE-Z arez. OUTE

RE-Zorse. OUT:

S1-Liqd. OUTE

S1-LEVEI. OUT

S1-Wa11. QUTE

SE-LEVE1. QUT

RE-ZGrEE. QUTE

LEDir I I OUTE

Ltir out. OuTE

FrotThW . OUT

Cnd-Levi. QUT

HOPFE' . OLT

Feeder. OUT

Mistarit. OUT
35.36

49. 12

50.39

IEZ.

1 2 a. 6

ext.

44.25

176.19

2.:. 19

1EE. 71

$50=.64$

EE: 3.01

0.00

$4=.08$

1106.50

200.00

e0\%.70 


\begin{tabular}{|c|c|}
\hline$E I$ & 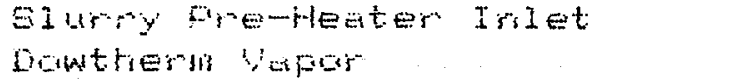 \\
\hline & Dowttiern Ligsiaj \\
\hline & 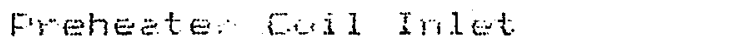 \\
\hline & Fretreater Coil Outlet \\
\hline & SI Hrir Fremedter Irlet \\
\hline & Dowtreners Vepocis \\
\hline & 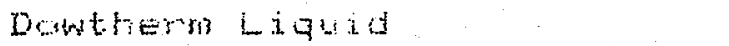 \\
\hline & Preheater $\operatorname{Cos} 1$ Irilet \\
\hline & Frenteaten Coil outlet \\
\hline & SI ury Pre-Hester Inlet \\
\hline & 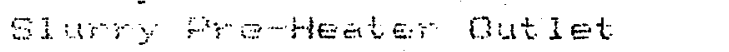 \\
\hline & Monutherrin Liquic Temperatire \\
\hline & Fyetrenter Cois IrIet \\
\hline & Fertater Goil Outlet \\
\hline
\end{tabular}

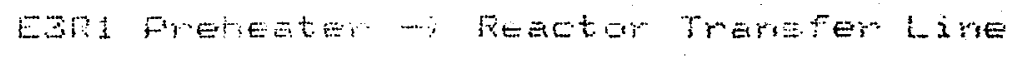

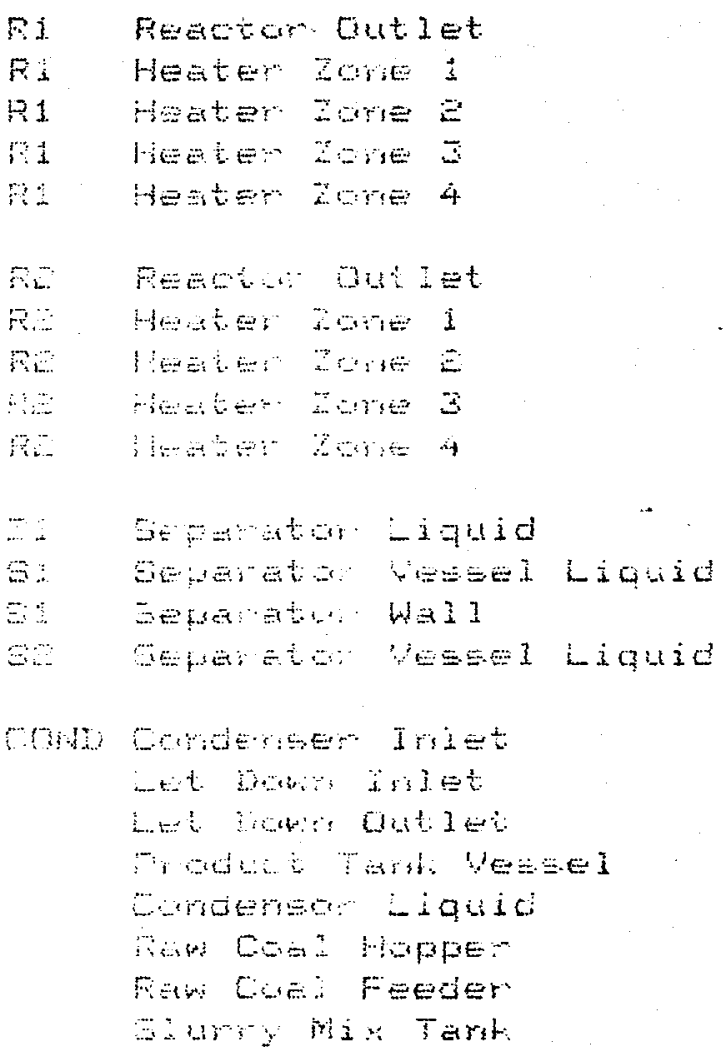

\author{
EETHFrES. DUT \\ EE-DUFTH. QUTE \\ EE-DLGTH. OUT:

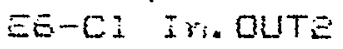 \\ EE-OIOUt. OUTE \\ EE-EEFY \\ EE-DVPTH. OUTE \\ Es-DLGTH. OUTE \\ EE-CI In. OUTE \\ EE-C1Out. OUTE \\ ES-EGFrE. OUT \\ EORTFHES. OUT \\ EG-DLGTI. OUTE \\ E9-CI In. OUTE \\ Eヨ-C.I Out. OUTE
}

1 $9205.5=$

3E.6.05

511.68

…․․ㄹ

375.43

$17=6.99$

546.47

547.10

$373 .=5$

506.75

E0. 2073.15

E47. 34

500. E:

615.34

EATRE XF. QUTE

617.59

RS-OtThP. QLITE

R3-Zare 1. DUTE

RZ-ZoreE. QUTE

R3-ZGrez. OUTE

Fב-zGre4. OLTE

6호는

617.30

617.38

$6: 1.35$

624. 95

REQT:

FE-z arei. OUT

RE- ZGMEZ. OLT

PE-zures. OLTE

Re-zirie4. QUTE

91-1. iqd. OLF

31-LEVE 1 . OUT"

S1-Wail. CLITE

SE-LEWE . OUT

25.35

47.91

49.59

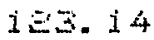

$1=3.77$

릭. 79

63.06

173.56

E. $4=$

R-Z-zreE. OUTE

$1=1.4=$

69.67

$=30.50$

a. 60

37.50

153.06

$=00,05$

$15 \% 1$ 
E: Slurry Fre-tieater Irlet

E1 Dantherm "apor"

EI Dowtherm Liquid

Ei Fretieder Cui Iriet

E1 Freheater Coii Gutlet

LE SIUr子y Fre-Heatei IrIEt

EE Dolitherom vajur

EI Dowthem Liguid

EE Treheater Coil Inilet:

E: Freneater Cail Outlet

E3 Elury Fre-Heater Iriet

E) Ii iny fremeater Qut let

IJ joltritierm Liquid Temperatire

E

IJ Frobeater Ecil Chitiet

\section{Esi meteater - Reecta Transfer Lire}

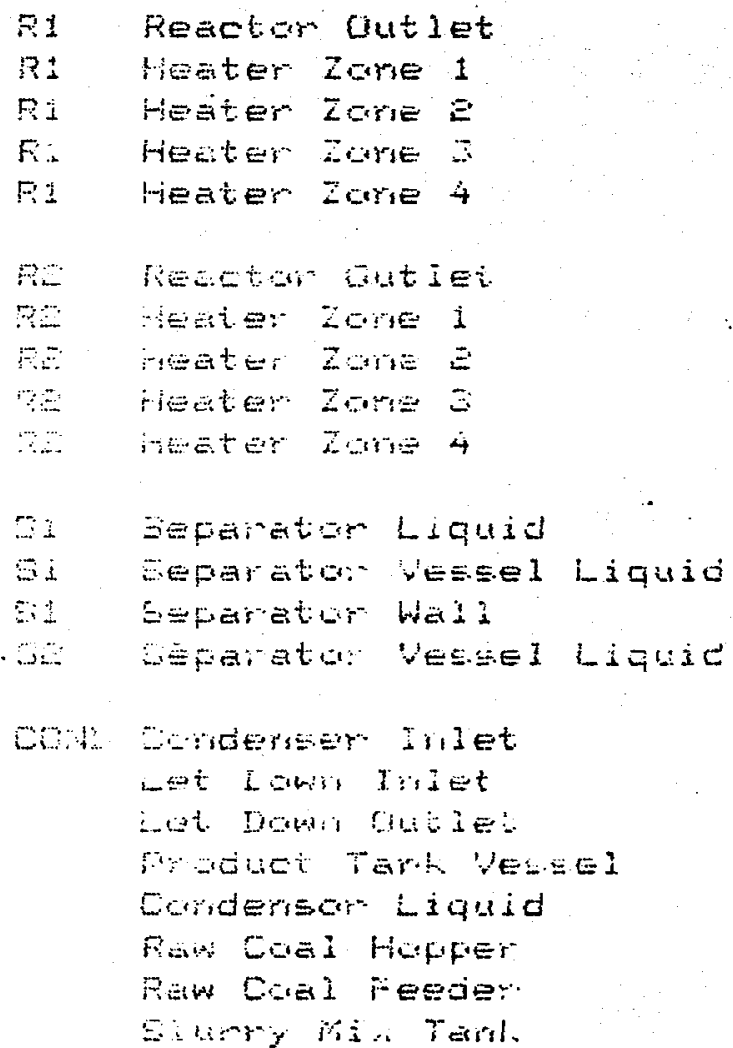

\author{
ESIrifires. QUTT \\ EE-DVETH. OUTE \\ EE-DLGTH. OUTE \\ EE-EX IN. OUTE \\ EE-Cidut. CuTL \\ EE-ESPIS. QUTT \\ EE-DVPTM. CUTR \\ ES-DLQTH. QUTE \\ ES-CI I R. QUTE \\ ES-CIOUt. QUTE \\ EST-ESFrS. OUT \\ ESOtFres. DUT \\ EG-DLGTW. DUTE \\ E9-CI Ir. QUT: \\ E9-CIQut. QUTE
}

ES-R3 XF. OUTE

R3-OtTmP. OUTE

R3-Zarie 1. DUTE

R3-Zaree. QUTE

RZ-ZarES. QUTE

F3-zarie4. OUTE

REQt THF. OUT

RE-Zare1. QUT

RE-ZOWEE. OUT

FE-ZCUES. DUTE

RE-ZUIE4. DUTE

Si-Ligd. OUTE

S1-LEVEI. OUT

Si-i山A 1 . OUTE

EE-LEVE1. OUT

FË-Zarie:. OLTE

LtDrs IT. OUTE

Ltor: Gut. OUTE

FrodTHL i. OLT

End-LEV]. OUT

Hofpe:. OUT

Feeder. Out

Mixiarik aUt
1757. $\%$

380.06

51‥ 91

23E. 54

$404 .=1$

¿693.

560.60

567.00

405.53

536.54

7

Eog3. $1=$

밍. 90

644.98

536.97

617. 6 .

619.48

629.48

613.01

EE1. 57

627.03

E33.73

34.49

47.55

49.31

IEE.

i $\mathrm{EN} .0$

EOS. EE

54.24

167.57

르.

1E0. $8 \mathrm{~s}$

$617.6=$

300.05

0.00

$37.8 \mathrm{~s}$

0.00

I 9 E. 7

51.61 
Appendix D

Process Development Unit (PDU) \#75

Date: 11/30/95

Feed Material: Thailand lignite slurry

Particle Size: -60 mesh

Feed slurry flowrate (lb/hr): 525

\section{Conditions}

temperature (degrees F): 575

set condition pressure (psi): $2100 \mathrm{psi}$

est. residence time (min): 15 minutes

Length of test (min): 90 


\begin{tabular}{|c|c|c|c|c|}
\hline 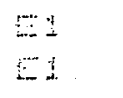 & 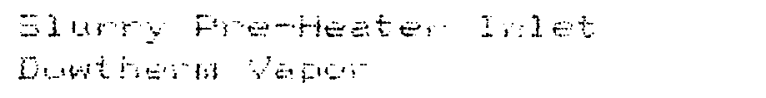 & & 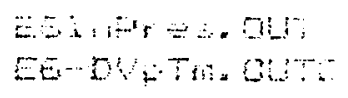 & $\begin{aligned} 394 \cdot 36 \\
3+2\end{aligned}$ \\
\hline$E i$ & Dowthern Liquid & & 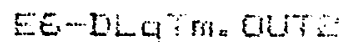 & $476, \%$ \\
\hline E: & 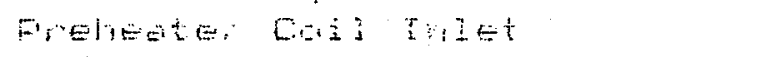 & & EE-CI I: OUTE & $E=E . \cdots$ \\
\hline$i$ & 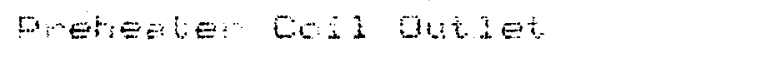 & & 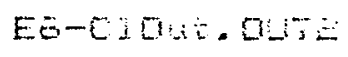 & $-3,-2.2$ \\
\hline & 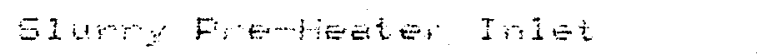 & & 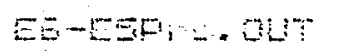 & 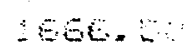 \\
\hline & Dowthern vanom & & EE-DVpr. OUT & $\exists \equiv \pm .+1$ \\
\hline & Downtism & & 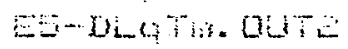 & $-2+2.06$ \\
\hline & Pretreater Cois indet & & EE-COI Irs. CUT: & 370.3 \\
\hline & Prefeate $C$ ois Gut $i=t$ & & 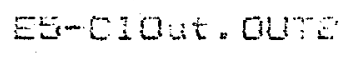 & $490 \times 3$ \\
\hline & Wishy Fro-Heater Injet & & 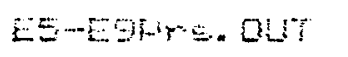 & 300 \\
\hline$\therefore$ & 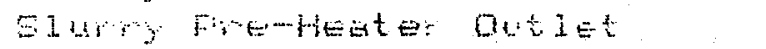 & & EOQtF $\xi^{2}=0$. aUT & 2030.43 \\
\hline$\Xi$ & Downthern Liquid Temperatue & & ES-1LGTh. USTE & $\theta 1 \equiv . \because 7$ \\
\hline 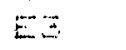 & Freherter Coin roiet & & EP-CI Ir. QUTE: & $49 \pm 3$ \\
\hline & Frelieter boil butiet & & E9-OSOut - CuTE & ESこ.こE \\
\hline 83 & 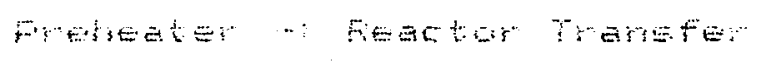 & Lins & EOW $x+$. OU. & $58+2.5$ \\
\hline & $\pi 0 t \operatorname{com} \quad 04 \div 1=t$ & & F $3-0 t$ Then aute & $=73.71$ \\
\hline$\vdots$ & 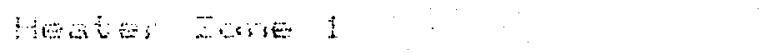 & & R3-2cores. DULE & 578.24 \\
\hline & 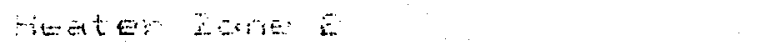 & & R3-zomeE. OUTE & 580.63 \\
\hline$\vdots$ & Men & & FS-ZGrez, OUTE & $59+51$ \\
\hline 1. & Hettor $\quad z, r=4$ & & R3-20rs ALT EL & 585.89 \\
\hline & nestug autit & & REot ThP. OUT & $17 \mathrm{E} \cdot \mathrm{a}=$ \\
\hline & Hester $20 \pi 01$ & & $F=-2 \mathrm{Cr}=1 . \mathrm{CUT}$ & $=72.76$ \\
\hline & 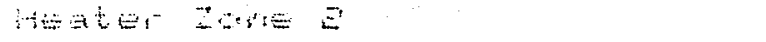 & & RE-ZOUEE = OUT & 254.67 \\
\hline & 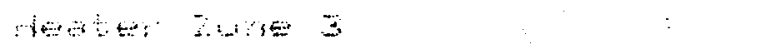 & & $R 2-2 \operatorname{cose} 3.0 \mathrm{OH}=$ & $44 E \cdot 53$ \\
\hline & Hente: 20 - & & RE-Zore4, OUT: & 331.17 \\
\hline & 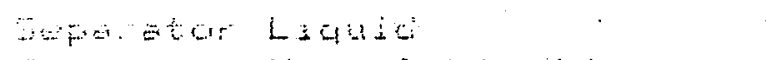 & & st-Liqo. OLT: & 107. \\
\hline & 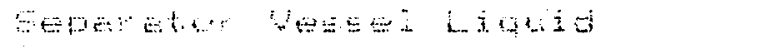 & & Bi-LEHE. QUT? & 44.13 \\
\hline & 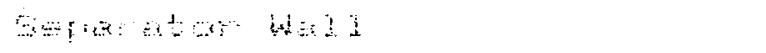 & & 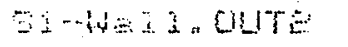 & $5 x+4$ \\
\hline & 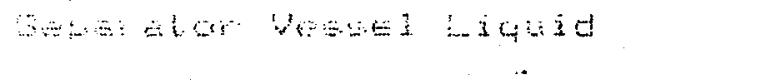 & & $S E-$ LE WE: DU & $E-20$ \\
\hline & Womenes: 3net & & $R=-2$ oreE. OUT E' & 490.4 \\
\hline & 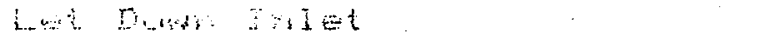 & & Lon; In OUTE & $301 \times 4$ \\
\hline & $\therefore \in t$ Dewn outiet & & Lter oxt ours & $3=-83$ \\
\hline & 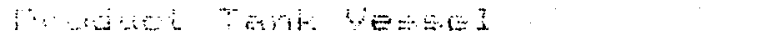 & & 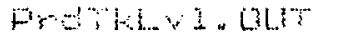 & 0.0 \\
\hline & 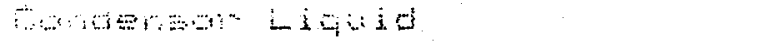 & & End-inew a d & 2.1 .1 \\
\hline & 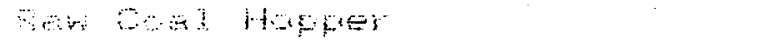 & & Hoper - our & \% \\
\hline & Now os I Feeder & & Feteder. OL & 176.00 \\
\hline & 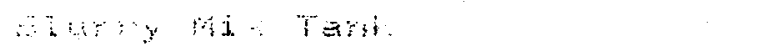 & & mis: antom: ont & $4 \ldots 2$ \\
\hline
\end{tabular}




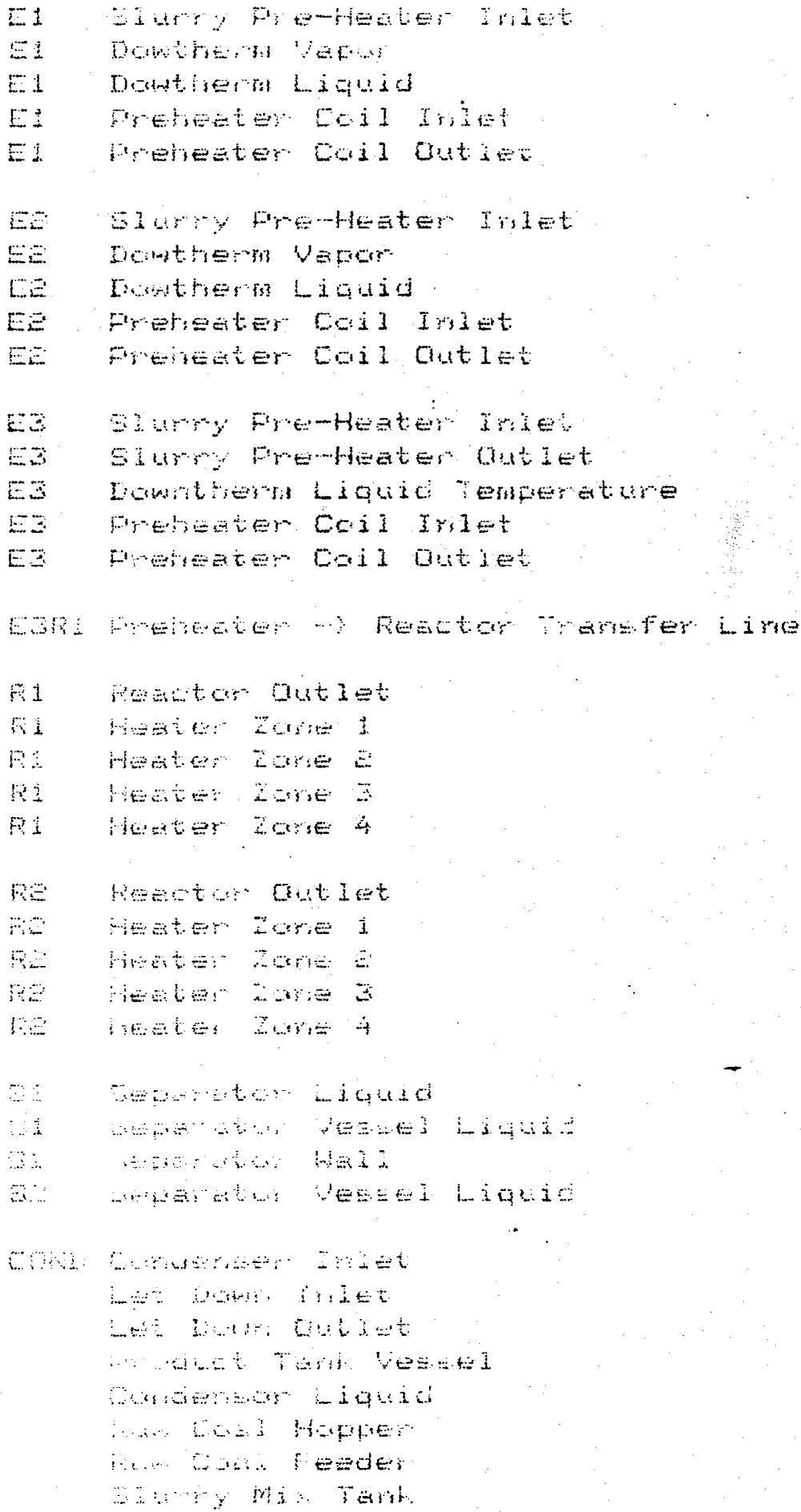

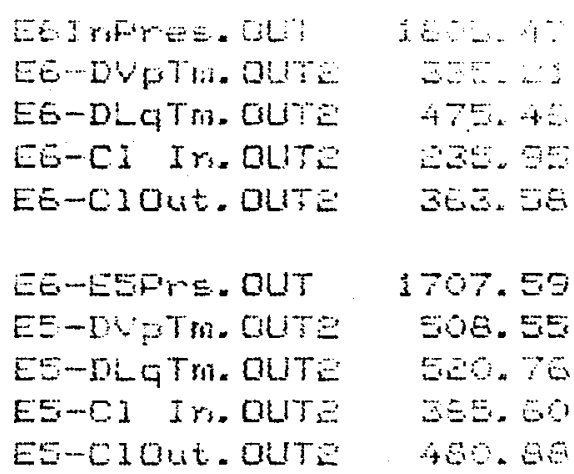

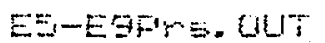

wgat Fres. aut

ES-DLOTH. OUTE Eg-CI In. OUT: Es-CICAt. OUTE

EG-FS Xf. OLTE

R3-OT:TmP. OLITE Rझ-ZGrEI. QLTE F-zorez. ULTE Fз-zores. OUT: RS-Zarté. OUTE

PEOt TIM D QUT

FE-Zarei. OUT

RE-Iorez. OUT

RE-ZCभES. OUTE

RE-ZGrE4. QUT:

6i-iaj. OUn:

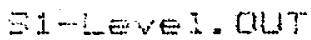

51-HEil. OUT?

B-imevit ou

a- arrez our

LIDrs Tr. QUTE

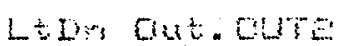

Fydrtis.

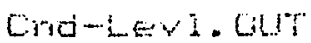

itopges. 04

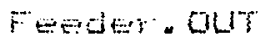

mis Tenthorir
290.75

2796.93

E10. 96

$48=7 \%$

$=30, \approx 3$

501.5

593.65

$5 B 01.1 \%$

응.

583.53

ㅍ․ 96

$=31.55$ $=99.81$ 30.19 560.13 50.15

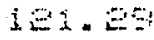
Bis. 5 99. 70

$\because+2.3$

572.45 50) 07 - 67.15

i.

46,8

$6=4.40$ सी. 0 $17 \% .10$ 


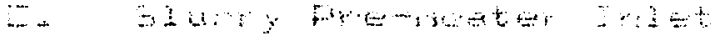

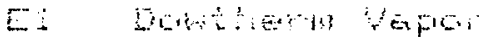

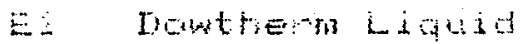

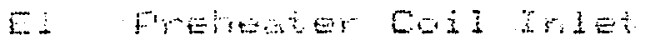

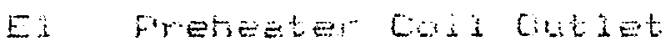

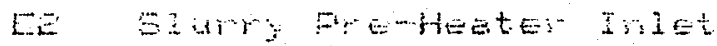

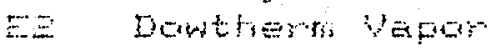

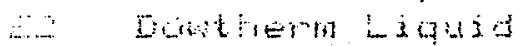

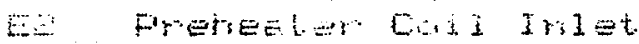

E- Fitheater buil Gutieb

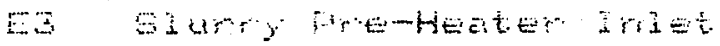

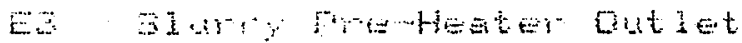

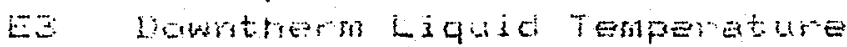

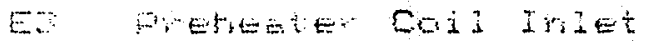

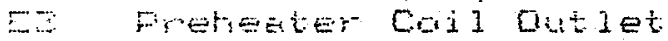

- 1 -

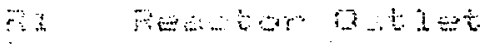

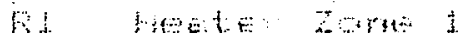

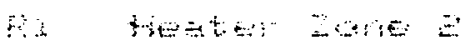

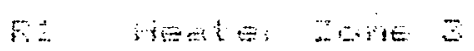

Ri Hoters zorse 4

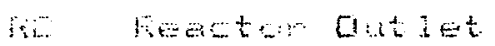

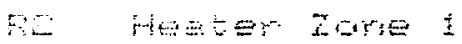

ne Histis are

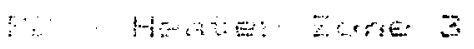

n M

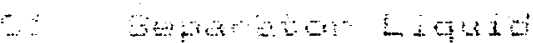

- $\quad$ b

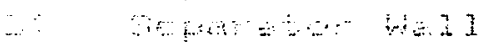

$\therefore \quad$ atom

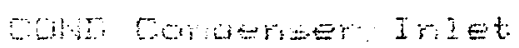

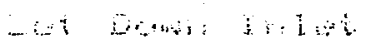

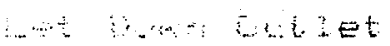

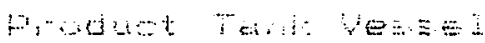

t.

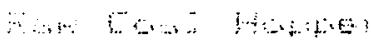

an Cras i

B.
Ex is a o a

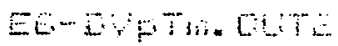

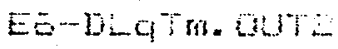

EE-CI IN Thin

ES-[JClut. OLT:

EE-EEF:D. OUT

EE-DVATH. GITE

BODLTM. QLTE

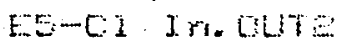

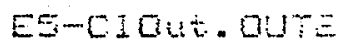

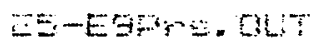

EgOtFrES. OUT

EO-DLGTH. QUTE

EJ-C. Ir. QUTE

Eق-C1Out. OUTE:

Lire

ES-Fa Xf. QUT:

F3-CITHP. TUT2

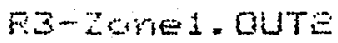

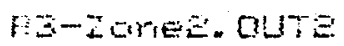

RJ-ZGREZ. QLTE

R3-y Gre4. OLT:

REQR THF- OUT

Fi-zares. OUT

RE-ZErieE. QUT

Re-zores. out $=$

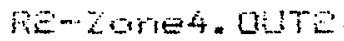

a-iqu. QUT:

Si-IEtEL . OLU

51-4-12. OUT?

G.

PE-Zura. ULT:

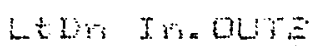

Lor ont on

Foग

5.

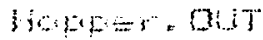

Fandos. alt

;

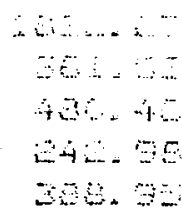

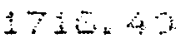

$I_{1}=4.84$

$5=7 \%$

300.95

$405 . \pm$

ㄴ.

Q69.

E.4. 75

उ० 34

580.79

583.97

505

궁. $E 1$

뎐. 95

$586.7 \%$

550.64

303.20

302.47

aIt . 0

581.79

sin. 39

+ 5.7

E. म

4.3. 9

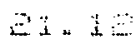

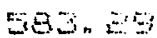

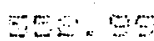

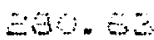

$\therefore \times$;

$-60+8$

$\therefore .50$

¿ 30.5

$\therefore \therefore, 40$ 


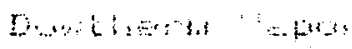

Dowtherr Liquid

$=1$

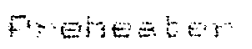

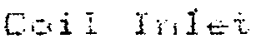

1.

Folvetter

Cois $042+2$

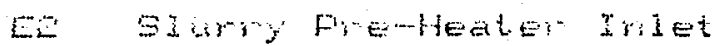

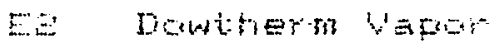

$E=$ Sowtierlis Liguid

EE Frehejter Coil Iriet.

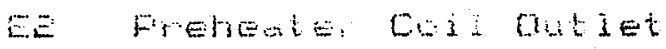

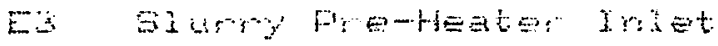

Ex $\quad$ in

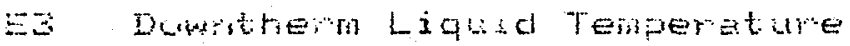

E. Prentester Coiz Injet

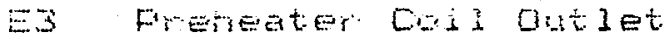

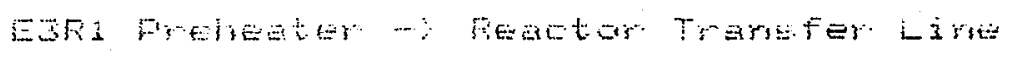

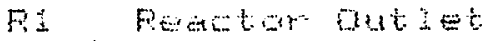

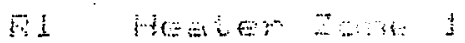

Fi Hetter Zore

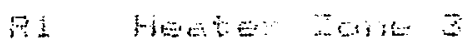

Pi Herter arm 4

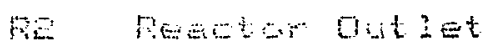

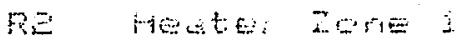

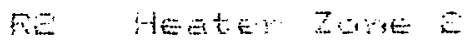

pe

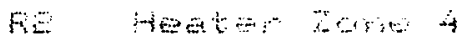

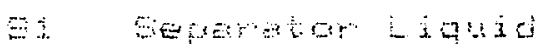

5 5 -

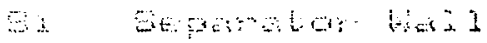

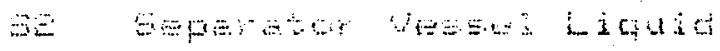

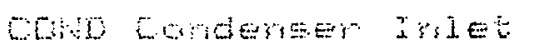

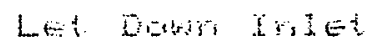

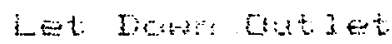

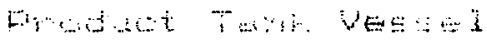

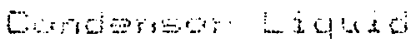

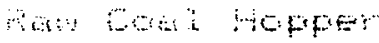

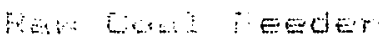

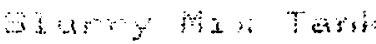

EE-TH

EE-DLG\% OLT:

EE-EI IH. OUTE

EE-CIOAt. OUTE

EE-CORM. OUL

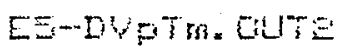

EET-OLGTH. CLTE

EE-OI Ir. OLTE

EE $[10 \mathrm{HE}$. OUT:

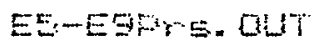

EGCHFrES. OLT

EG-DLGTM. OUTE

EG-Cl Ir. OUTE

EP-C.1Gut - DUTE

EG-RS KF.OUTE

Fis-ot Thp. Qure

R3-Z GUE DUTE

F-Z-ErreE. OUT:

R.3-zGHE 3 . QUTE

R3-zarie 4 OUTE

REQt ThP. OUT

BE-ZOre1. OUT

RE-ZZURE. OUT

Fi-AGHEZ. OUTE

Fin-zore4. OUTE

Gi-Liqd. Qurtis

SI-LEvel. OUT

61-6al1. 0410

6-...

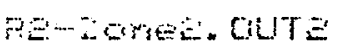

LeJir $x$. QUTI

Ltirs out cours:

FHeTkL i. OUT

Cro-LeVI. GUT

Hopes an

f $t=d e r$. OUT

Mis mat. out
$32 \div$

$+2+\frac{1}{2}=-4$

讷比 7

40.95

1695.90

$540,4=$

5if $\because 3$

$402 .+5$

$\because 15.5$

$=034.15$

2047.03

ESE: is

5.E(0.7

54.51

594.35

593.65

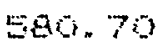

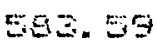

587.14

595.65

307.65

363.54

305.19

585.90

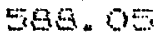

100.5

3.77

114.51

$\because 1$.

5.86. 97

EE.

$=02.9 \%$

0.0

30.

Estom

ज्ञात,

mit. 7 


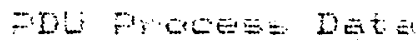

$12: 06$ 1. 1300

Ei El wry Fre-fedter indet

ESIrFres. QUTT

มงเ.

E1 Dowtherra Vafor

EI Dewtherm Liquid

EL Frebeater Cois irlet

Ei Foheater ouil butiet

EE-DVPTH. DUT:

578. E.

EE-DLQTM. QUT:

485.95

ES-EI I M. QUTTE

251.15

EE-Clout. OUtr:

400.7

ED Blury FreHeater Irlet

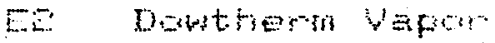

EE Dowthern inguid

Ee Fineheater Cois Imist

ED Freheater Coil Dutiet

ED Sinry rre-Heater iniet.

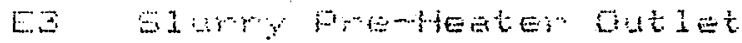

re vewation Liquid Tenperatur

Es Freheater Coil Tritet

E. Preteder Cosi Qutiet

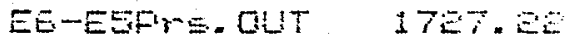

EE-DUPTM. OUTE EEA. 05

EE OLGTH. CUTE 5EN

EE-CI In. QUTR 400.64

EE-CIOLT. OUTE 530.96

ES-EGFr. OUT EOBE. ES

E90tFres. OUT EOQD.

EGMLATH. OUTE 594.03

E9-CI Tr. OUTE 53.13

ES-CiQut. OUT2 E7E.09

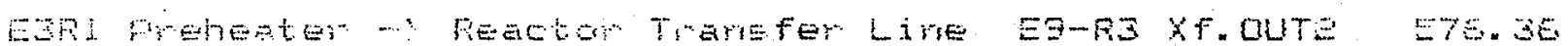

Bi Fencom outlet

R3-OT Trop. OUTE

590.53

R3-Zcrie1. OUTE 573.49

R3-Zaree: QUTE 574.5

R3-ZOMEZ. QUTE 581.26

PS-Zorre4. OUTE 591.55

Re Senton OAl

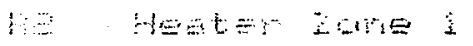

REQtTMP.OUT 31E. 94

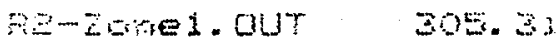

FE-ZWHE. OUT 310.5

RE-ZGES. OUTE ES4.

ne-Zare4.0UTE E94.46

Hereter zore it

S1-Liqd. OUTE

195.91

81-LEve1. OUT

ED. $\times 4$

S1-He12. DUTE

i 53.30

SE-LEVE1. DUT

21.45

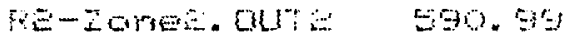

Ltro Ir. QUT 500.91

Lt.Dr gut. ULte $24 \% .3$

FrdTl-D. OUT

$\therefore .0$

Crod-LEWI. OUT $45.9 E$

Happer. OUT

Feeder. Olir

430.05

200.00

MinTarito OUT

150.38 
Appendix D

Date: 11/30/95

Process Development Unit (PDU) $\# 75$

Feed Material: Thailand lignite slurry

Particle Size: -60 mesh

Feed slurry flowrate (lb/hr): 525

\section{Conditions}

temperature (dègrees F): 620

set condition pressure (psi): $2100 \mathrm{psi}$

est. residence time (min): 15 minutes

Length of test (min): 90 


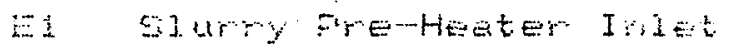

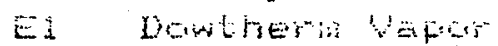

El Fowatherm Libaid

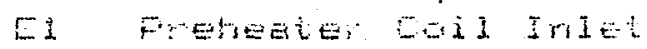

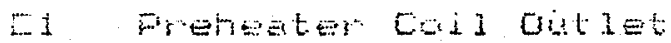

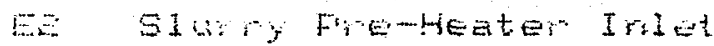

En Dowthers befor

Ea Drintrem Liquid

E2 Frentater Cois Iriet

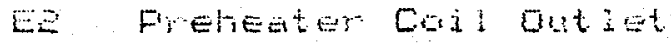

E. Sl rry pretreaker irlet

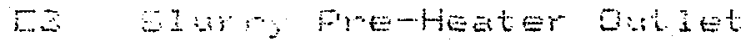

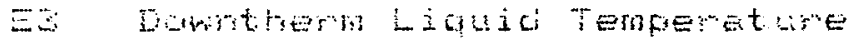

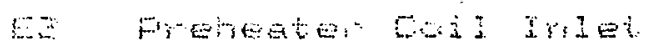

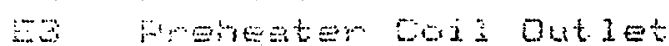

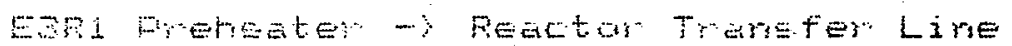

F: Pétom dut let

Ri Htater Zare 1

Pi HEEta zorse

P1 miseter $=0$

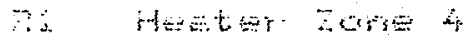

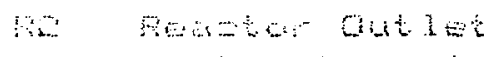

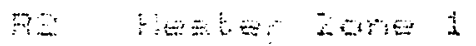

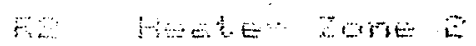

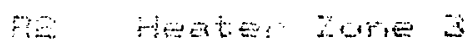

तो

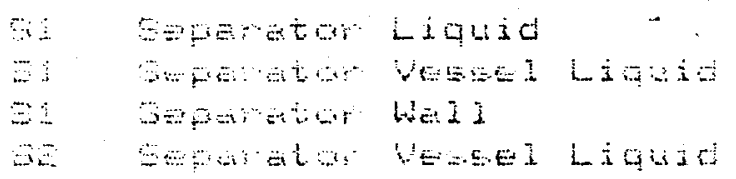

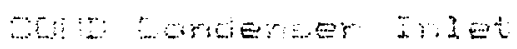

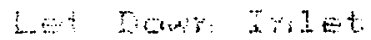

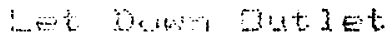

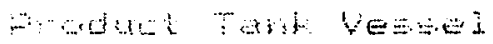

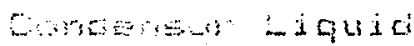

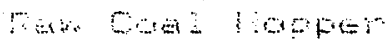

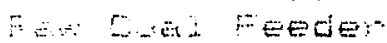

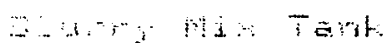

EEIMFrES OUT

ES-DVFTM. OUTE

EE-DLGTH. OLTE

EE CI Ir. OUT:

Ee-Cidut. Oure

EE-EEFTE- DUT

EE-DUTH. EUTE

Es-OLGTA. OUTE:

EE-CI IR. OUITE

EE-C10ut. OLITE

ES-EGFYS. OUT

E9QtFres. OUT

EG-DLGTS. OUTE

E9-CI IR. OLTE

E9-ciout. ouTE

E9-R3 XF. OUTE:

RS-OtTMP. OUTE

RE-Zore 1. OUTE

R3-ZareE. QUTTE

R3-zarez. QUTE

FA-Zarse . QUTE

REQt Tmp. OUT

RE-Zarsej. OLT

FE--zoree. UuT

RE-ZOrE B. DUTE

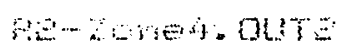

BI-LiqA. QUTE

SI-LEVE. OUT

S1-beli. OUTE

EE-LEVEI OUT

Fe-zereE. QU $=$

LED In. OUTE

LED GH OLTE

F:CTH:L. OU:

crod-1Exh. our

HOFFE: OLT

Feedes", OUT

Mintarta our i.912.

$27 \% .4$

$43 \% 49$

246.91

$200 . \therefore 0$

$17 i+10$

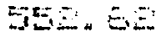

559.78

355.38

525.69

0074.51

0074.05

E45. 57

500.3

621.45

EะZ. 07

EEE. 11

E1. 43

600. 14

E⿺辶. 37

$6 \pm 5 \times 13$

310.

उ).

39.6

EOS. 8

$90=2$

Q13.4 4e. e?

103.

ㄷ.

00.3

570.99

롱두 19

$\therefore 0$

43.31

0567.01

500.

$876 \times 4$ 


\begin{tabular}{|c|c|c|c|c|}
\hline$E$ & 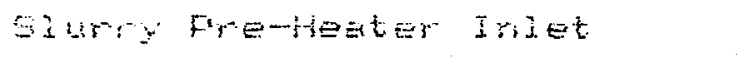 & & EEIrGYES. OLU & $151 i .3$ \\
\hline$=1$ & Dowthern tofero. & & ES-DVTH. GUTE & $\Xi E B .9 i$ \\
\hline$E I$ & Dowthym Liguid & & EE-DLGTH. IUTE & 457.54 \\
\hline$\therefore i$ & FinEGEter Coil Irist & & EE-CI IM.OUTL & 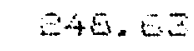 \\
\hline 1 & 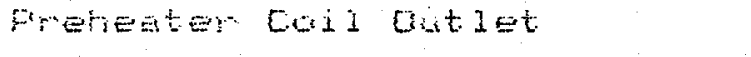 & & $E E-C i G t+0, E$ & 30.20 \\
\hline & 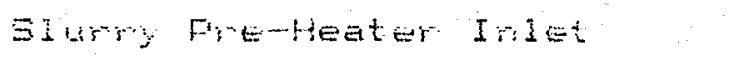 & & $E E-E D \%=. Q L T$ & 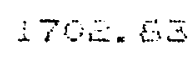 \\
\hline$\ddot{z}$ & Incwthen Vapur : & & EE-DVuTn. GUT: & $54:-45$ \\
\hline$a$ & Wuthbern bigiad & & EE-DLOTH. OUM: & $5 E \cdot \theta$ \\
\hline$\because$ & Fretretsen Coil Iriet & & $E=-C 1 \quad I+1.0 L Y=$ & 395.58 \\
\hline$\therefore$ & Premeater Coid outidet & & EE-CIGHE. OUTE & $\dot{4}=7$ \\
\hline 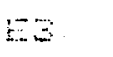 & S- try preptenter Irist & 3 & $E=595=04$ & $=564.70$ \\
\hline 3 & 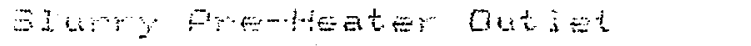 & & EgQtERE QLET & $=050.46$ \\
\hline$\because$ & 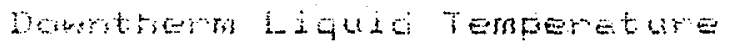 & & $E \exists-D !-9$ TH. DUT: & 643.45 \\
\hline 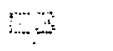 & 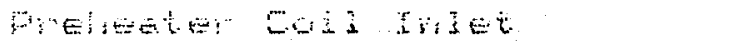 & & ES-C1 Ir. OLITE & 517.12 \\
\hline 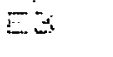 & 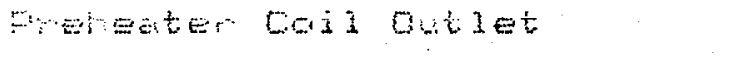 & & E9-0 a cut. aure & $4.5 i$ \\
\hline 20 & 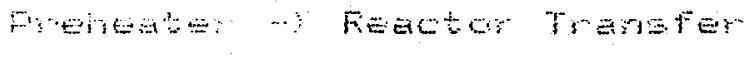 & Ling & $E 9-F=x F$. QLT: & 615.41 \\
\hline 3 & 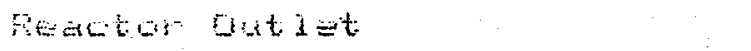 & & FЗ-DETImP. CULTE & 631.97 \\
\hline 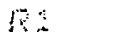 & Hedter $x$ ate 1 & & RA-Zsries- QUTZ & $E 11.39$ \\
\hline$\vdots$ & Hedter Zarse E: & & 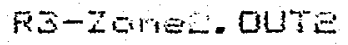 & 615.67 \\
\hline $\overrightarrow{1}$ & 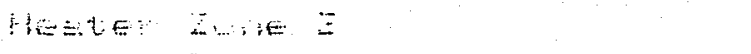 & & $R \Xi-205 E 3 . Q U T=$ & 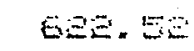 \\
\hline 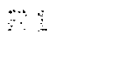 & 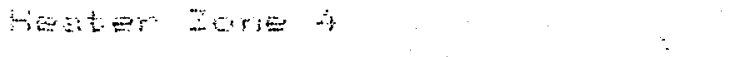 & & R3-Zcried. QUU: & $63 \pm .77$ \\
\hline 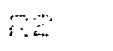 & 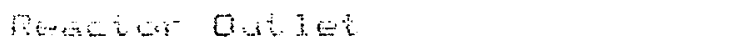 & & PaDt THF. QLT & 238,10 \\
\hline 6 & WE & - & VE-Zarses . GUT & $3 \pm E .71$ \\
\hline 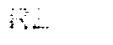 & 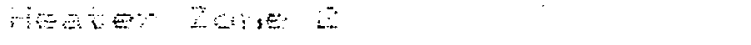 & & $F=-20 \%=.04 \%$ & 30.39 \\
\hline$\therefore$ & 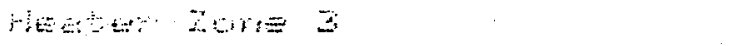 & & RE-ZCHE & $6=3.40$ \\
\hline$\because$ & 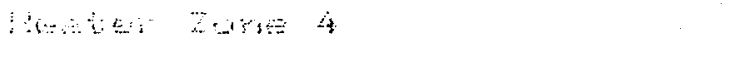 & & 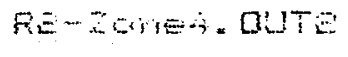 & $E=i .5$ \\
\hline & 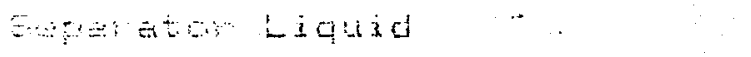 & & $\because 1-i .90 .047=$ & $\Xi \dot{a}$. \\
\hline & $\because$ mbla & & 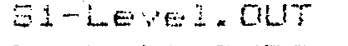 & $3 \%+2$ \\
\hline & 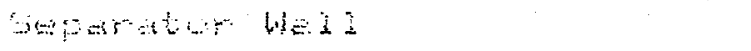 & & $\because 1-W=11.047 a$ & IE. SE \\
\hline 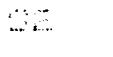 & $\because$ снд & & 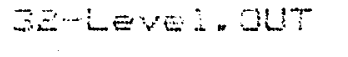 & 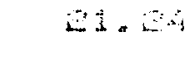 \\
\hline$\sqrt{3}$ & 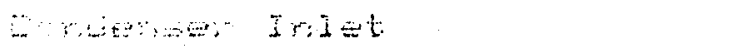 & & $F=\cdots 2 D=E 2017$ & $E=-5$ \\
\hline & 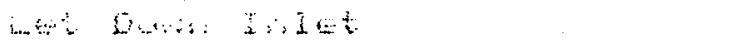 & & 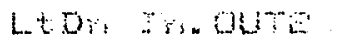 & 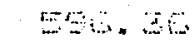 \\
\hline & 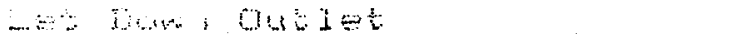 & & 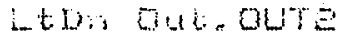 & 545.5 \\
\hline & 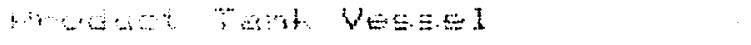 & & FodThLY. OHT & \\
\hline & 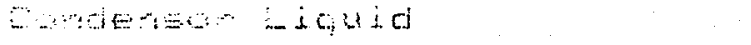 & & Gid-LEx "OUT & $\therefore \because n$ \\
\hline & 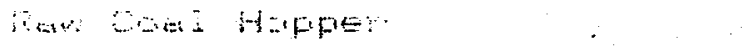 & & HWFFE . OUT & $\therefore E=-30$ \\
\hline & 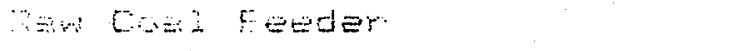 & & Feder. Qu" & $=00,00$ \\
\hline & 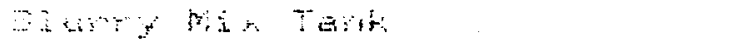 & & 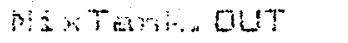 & 275.0 \\
\hline
\end{tabular}




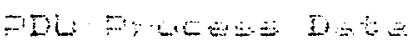

$10: 00$ म. 20:3

E

E1 Dowthem lafw

El Fretienter Cois rilet

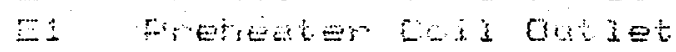

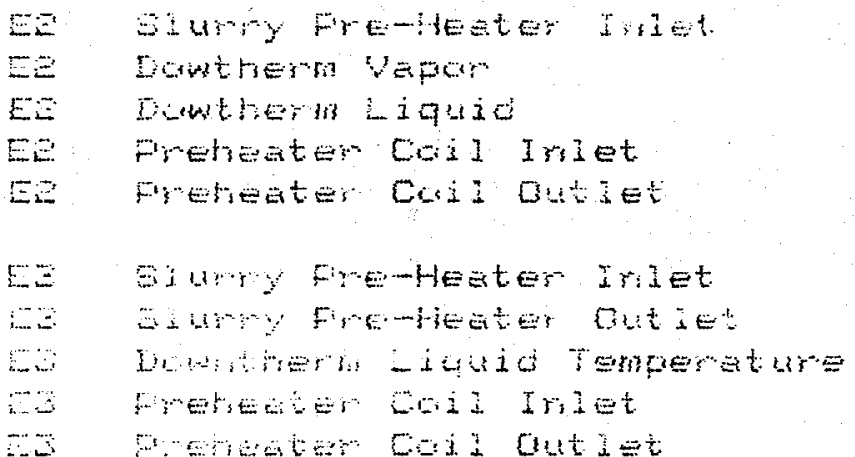

EEInFres. QUT

EE-DViTH. OUT:

EE-DLGTh. OLTE

EC-CI IY. QUTE

EE-Cidet. cute

EE-EDFTS. QUT

EETHPTM. OUTE

EE-DLGTH. OUTE

EEI CI I I . QUTSE

ES-Clout. OUTE

EE-ESFr $\equiv$. QUUT

EGOtFres. OUT

E9-DLQTH. OUTE

E9-CI Ir. DUT:

E9-CIOUT. OUTE

ES-RE XF. OUTE

R3-citTmp. OUTE PRZ-Zare 1. OUTE Fis-zareE. OUTE RS-ZGrES. QUTE FS-ZEREA. OUTE

FEOT THF - QUT

RE-Zare1. QUT

RE-ZCMEE. DUT

Re-zorez. OUTE

RE-ZEMEA. OLTE

S1-iqd. OUTE

31-LEven. QUT

S1 -.Maji. OUTE

BE-Level. OUT

FE-FWEE. CLTE

Lito Fr. OLTO

Lt. Dr ost. Oure:

Mrothini. OUT

Crid-Lewl. out

Hopper OUT

$F=E d s \%$ OUT

MixTant. OUT
1773.05

414.14

514. $\equiv 4$

190.46

409.37

1700.12

574.00

579. 54

41.95

547,34

$2069.1 \%$

อरes. 39

$544.9=$

54. 69

613.90

600.49

6:9.09

616. 25

EIE. 95

520.69

609.06

$330.1 \%$

उЕ. 5

3อง.

EE. $\theta$

ED. 0

E39. 5

$49 \times 47$

IE⿰ 39

50.94

EE: 15

001.80

CEE.

3.

43.57

1909.23

200.00

E96. 


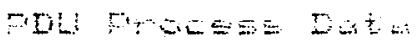

1E: 15 11:30:0

\begin{tabular}{|c|c|}
\hline EI & 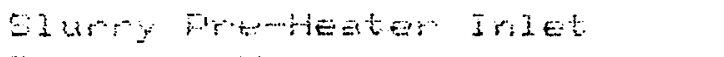 \\
\hline 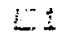 & 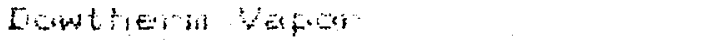 \\
\hline$E:$ & Dowtherm ingeide \\
\hline$E$ & Freneater Goi I Inat \\
\hline & Fretheder Coil dutset \\
\hline & 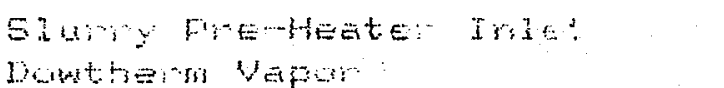 \\
\hline & Dowthes biquie \\
\hline & Frohester Coil Iniet \\
\hline & Freheater Coil Outit. \\
\hline & Sicury frethester IrIet \\
\hline & 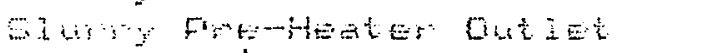 \\
\hline & Dewriberm'iagid Temperature \\
\hline & 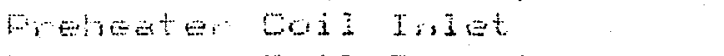 \\
\hline & \\
\hline
\end{tabular}

EEIrFtres. OUT

EE DUpTh. OUT:

EE-DLGTH. CUTE

EE-CD TY. OLTE

EE-CEOHE. OUTE

EE-ESFR. OUT

EE-DUpTH. QLTE

ES-OLGTh. OUT:

ES-CI Ir. OLTE:

EE-C1Out. OUTE

EE-EGFHE. QUT

EgQtFines. OUT

ES-DLGTH. OLITE

E9-CI Ir. QUTE

ES-C1Out. CUTE

ES-P3 XF. QUTE

RS-QtTME. OUTE

R3-ZarEA. OUTE

FI-ZareE. OUTE

FS-ZCUMEZ. DUTE

PS-ZGreA. QLTE

PEOT Thp. OUF

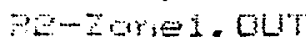

ne-zomeE. QU?

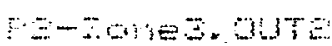

ne-zont a du:

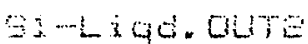

Si-imerez. OLT

E) He i. Otre

S... neved. MU:

PQ-2OMEE. OUTE

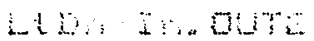

EDa gut anti:

PaTHLY1, OLT

CFA-LEY. OU:

Hopfes - abr

Feeder. on

materst. OL?
1815.69

궁. 30

494.49

195. 35

369.43

270.95

567.73

도. 74

371.80

5ㄷ.7은

EOSE. ES

EOE. 90

$64 \div .7 \%$

500.50

$01 \Xi .85$

634.67

$E=4.96$

ब10.06

615.05

$6=0,44$

E24. 84

329.95

$3 \pm E .30$

उEE. OQ

ㅎ․․ 13

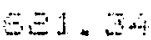

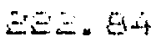

5. 69

17. 7 , 7

$\ldots+9$

E19.73

$-6.63$

ET⿱

$\therefore 00$

44.74

425,09

20.00

144,3 


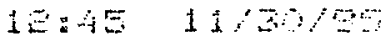

\begin{tabular}{|c|c|}
\hline$E 1$ & 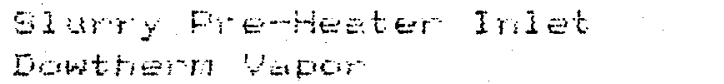 \\
\hline & Dewthern lalquid \\
\hline & Frebeater Golit Inet \\
\hline & Frethents cois Outiot \\
\hline & 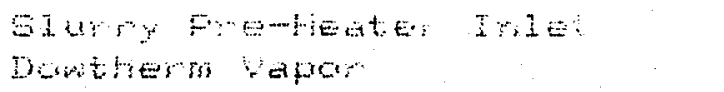 \\
\hline & 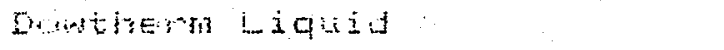 \\
\hline & Fretieater Coil Inlet \\
\hline & Frebeater Ooid Gutieb \\
\hline & 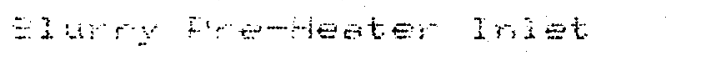 \\
\hline & 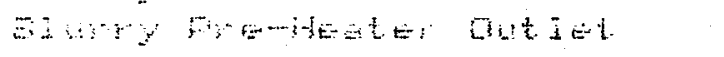 \\
\hline & 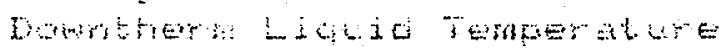 \\
\hline & 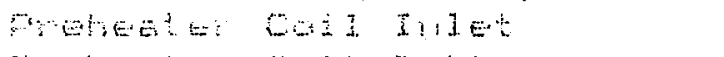 \\
\hline & 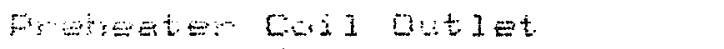 \\
\hline
\end{tabular}

Eg-R3 Xf. QuTE

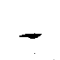

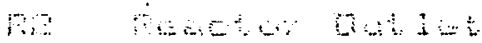

02 hester $=$

10 मrte

Qर कen zare 3

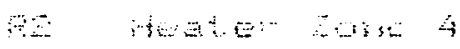

\begin{tabular}{|c|c|}
\hline $2 i$ & 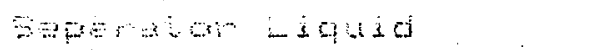 \\
\hline$=i$ & 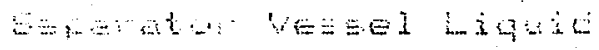 \\
\hline & 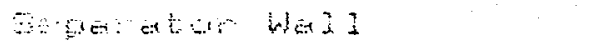 \\
\hline & Separaby yeded Liandel \\
\hline $3 \times$ & $\sigma a d n e$ : \\
\hline & 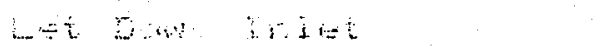 \\
\hline & 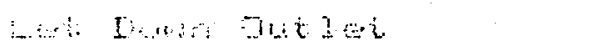 \\
\hline & 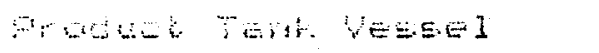 \\
\hline & Wracenes Licidid \\
\hline & 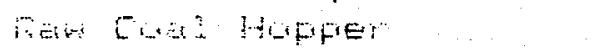 \\
\hline & 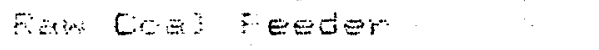 \\
\hline & Olu\% Mis Tams. \\
\hline
\end{tabular}

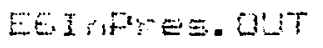

EE-DYTH. QLTE

EE-DLETH. QUTE

EE-EI I $\%$ QUT:

Es-Cout. OLTE

EE-ESF $=$ au?

EE-DVPTH. CUTE

EE DLGTH. RUTE

ESCI in. OLTE

EE-CIOUt . OUTE

EE-EGFr. OUT

ESOHFWE- DUT

EE-DLGTm. Oute

ES-CI Ir. OUTE

ES-CIQUt. GUTE

P.Z-OtTmP. OUTE

RE-zGNE1. OUTE

FE-ZareE. QUTE

RE-ZMrE. OUTE

R3-Zure4. OUTE

1750.55

$40=41$

499.34

EE. 20

$4 \pm E$.

$10 \%, 50$

572.77

5ita. 9

423.79

547.46

300.41

E0. $7 ?$

043.01

돈.5. 47

- EEI. ${ }^{4}$

6E1. I

$6=8.76$

616.46

E19.31

Eอs. 63

E30. Ei

30.23

330.34

320,75

6E5. 95

6.5. 80

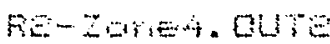

$\equiv 0.50$

$6 \mathrm{i}, 7 \mathrm{7}$

107.44

20.60

027.30

E16.14

$304.1 \%$

0. 0

30.3

on

Eक

5e. 63 


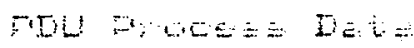

$13.00 \quad 1120090$

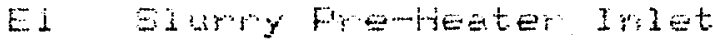

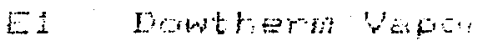

E: Dowtion Lighij:

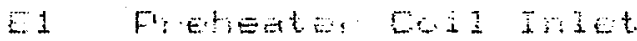

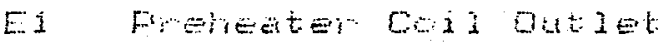

\begin{tabular}{|c|c|}
\hline$E$ & 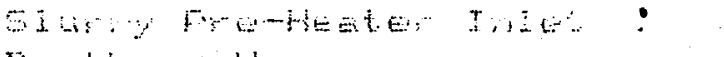 \\
\hline$E$ & 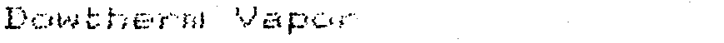 \\
\hline$E$ & Dothers - iatio \\
\hline$m$ & muthester -Coij Indet \\
\hline & 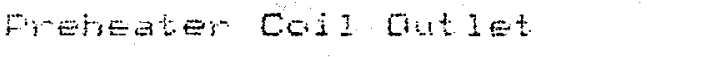 \\
\hline & $924,5 y \quad$ r \\
\hline 1 & 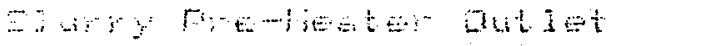 \\
\hline & 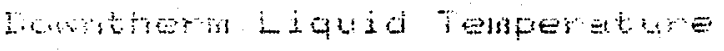 \\
\hline & 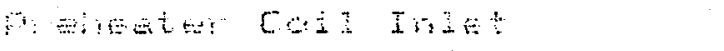 \\
\hline & 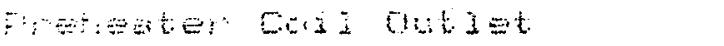 \\
\hline
\end{tabular}

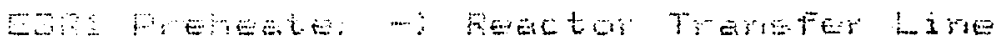

\begin{tabular}{|c|c|c|}
\hline & Penctar & $04 t=$ \\
\hline & $\operatorname{arcs}=$ & $Z$ OB⿴囗十⺝ \\
\hline & 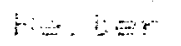 & $\therefore, 1=$ \\
\hline & Hots & $Z 01910$ \\
\hline & 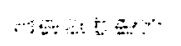 & $20:=0$ \\
\hline
\end{tabular}

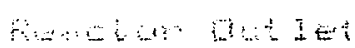

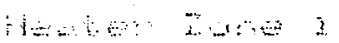

Hin a

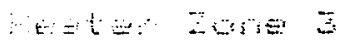

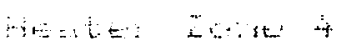

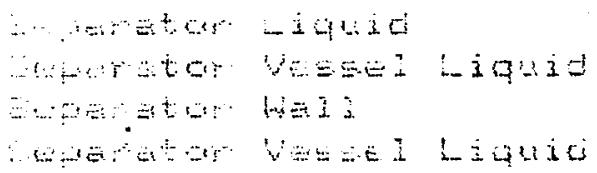

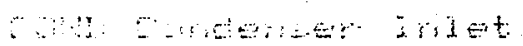

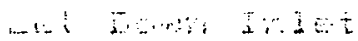

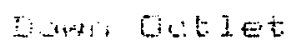

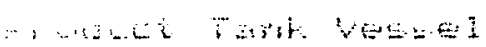

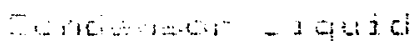

$\therefore$ A

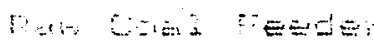

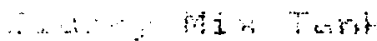

EEIRFHES. DUT

EE-DYFTH. CUT:

E-MLGTII. OLIT:

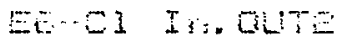

ES-CiDut. OLTE

EE-ESF:

Es-DVGTH. QU⿴囗十

- ES-DLGIm. OUTE

EE-CI Ir. OWT:

EE-CI Gut. OLTP

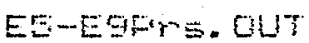

EgDt.FEE. GLIT

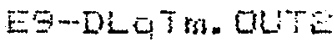

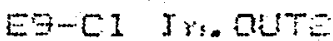

E9--EI Gut. OLTE

EORS XF. QUTE

F3-OLTmp. QUTE

$R=-20 \% E 1$. QUTE

F. $3-2$ GreE. OUTO

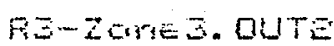

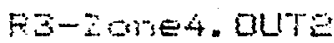

- FEOt The. RuT

DEN

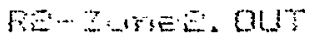

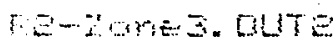

RE-2arse- QUT

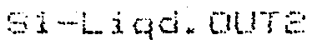

S1-LEVI. OUT

31-WEI3. CUT:

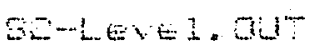

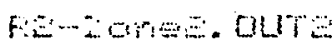

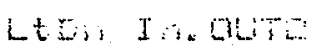

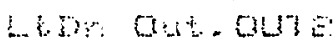

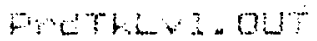

CHO-LEVI. GUT

Howpes. OUT

Feedy. OUIT

Fों:
I7E 7 .

$4=5=40$

프. 75

E: $\because$

$-4+4=6$

$16 E$. O

$50-91$

59. 10

443.65

ह57. 94

E0E. 25

ज्ञात

$54=95$

573.5

E⿺辶千 74

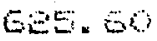

631.26

619.03

हE1.07

624. 62

E3E. 34

35.35

$3 \pm 6.54$

$336.3 \%$

EE. $7 \%$

6EA. 70

Q0. $E \%$

उ). $\cdots$

165.9

$20.5 \mathrm{Z}$

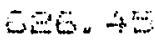

593.30

$=60.4$

$\therefore 0$

46.45

i.

0.00

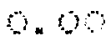


Appendix D

Process Development Unit (PDU) \#76

Date: $1 / 11 / 96$

Feed Material: Thailand lignite slurry

Particle Size: -60 mesh

Feed slurry flowrate (lb/hr): 525

\section{Conditions}

temperature (degrees F): 620

set condition pressure (psi): $2100 \mathrm{psi}$

est. residence time ( $\mathrm{min})$ : 7 minutes

Length of test (min): 180 
Wu mroces= Data

$13=3001 / 1296$

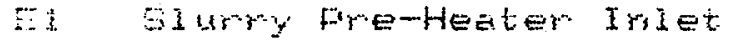

I. Disutherm vafur

El Dexthern Liquid

El Fretreater Cois Tritet

EL Fretrenter Coris Qutiet

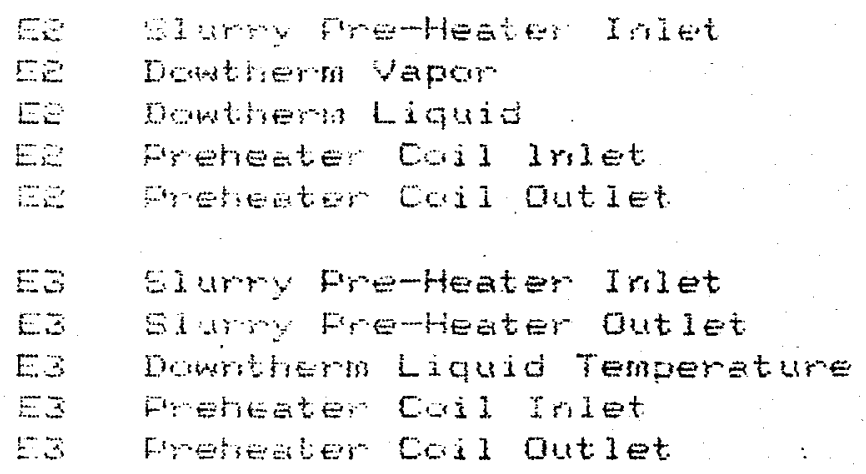

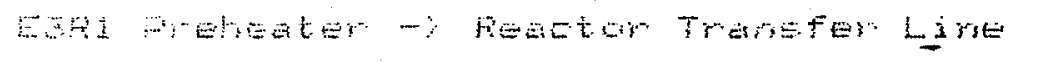

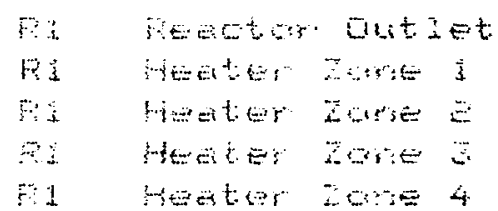

Po

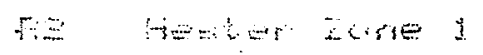

n Fut

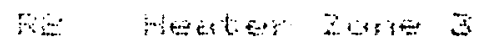

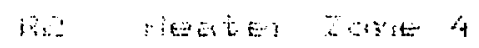

\begin{tabular}{|c|c|c|}
\hline$\because i$ & $3=$ antar Liquid & \\
\hline$\cdots$ & 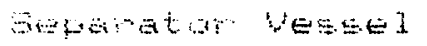 & Licuid \\
\hline 3 & 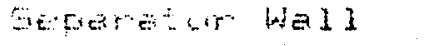 & \\
\hline- & 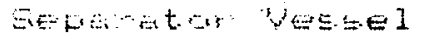 & Li44Uid \\
\hline \multirow[t]{8}{*}{ En: } & 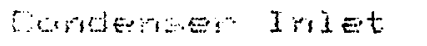 & \\
\hline & 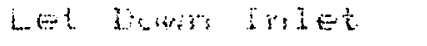 & \\
\hline & 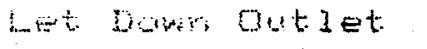 & \\
\hline & 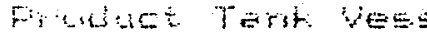 & $E 1$ \\
\hline & 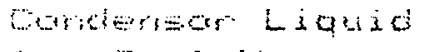 & \\
\hline & 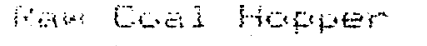 & \\
\hline & 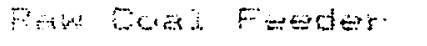 & \\
\hline & 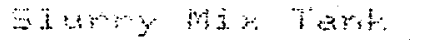 & \\
\hline
\end{tabular}

EQI THEE. QUY

EE-DVPTH. QUTE

EE-DLQTn. QLTE

EE-CI Ir. OLTE

EE-CI QUt. RUTE

EE-ESFYS. QUT

EE-DUPTI. OUT:

EE-DLGTM. QUTZ

EE-C: Ir. OUTE

EE-CI Dut. OUTE

Eㄷ-ESFHS. OUT

EGOtF'

E9-DLGTM. QUTE

59-EI Im. QUTE

ES-C] [ut. OUTE

EF-RE $x+$ OUTE

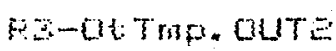

RZ-ZGrE 1. QUTE

6а-

F3-zores. OU7E

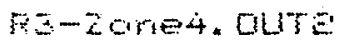

REOUT THE. CULT

Fu-Zori. OUT

FE- Z Wre

F.-

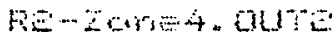

$51-1$ iqd. OUT:

SI-LEVE. DUT

5i-he 13 . GuT:

SE-LE

FE- - - M

Lt. Dh $7 \%$ OLT:-

LtDri Gut. OUTE

FrdThLV1. OUT

Crot-LEW . CUT

HoFbE , GUT

FeEder. QUT?

MixTart. OUT
$175 \% .96$

350,24

499.3

E4+ 0.00

40三. 至王

$165 \overline{6} .95$

$541.3=$

547.09

401 . E5

517.77

201E. 39

2000.94

637.76

50.03

년.

614.95

Q12.01

604.23

606.91

E11.95

618. 5

43.33

30. 27

Ea. 94

9i. 93

91. 3

193.02

39.91

130.45

$=0.93$

84, $=1$

$59 x .50$

ag. 11

0.00

$44,2=1$

270

$=00.00$

39.7 
MDL Fratos Deta

$1+450$ O

\begin{tabular}{|c|c|c|c|c|}
\hline 1 & 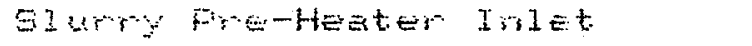 & & EEI T,FrES OUT & 1815.97 \\
\hline 2 & Poththerbis Vafus & & EE-DVPTH. OUT: & $37 \overrightarrow{2} \cdot 3$ \\
\hline & Denthithn Liquid & & EE-DLGTH. QUTE & 508.08 \\
\hline & 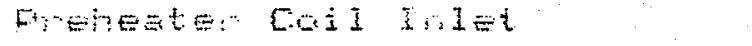 & & EE-CI Ir. OUT: & 255 \\
\hline & Hy ententer CoiI Citilet & & EE-CIDut. QUT 2 & 418.58 \\
\hline & 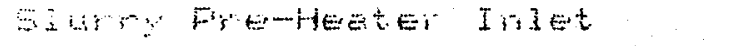 & & EE-ESFr.. DUT & 1719.6 \\
\hline & Dosthen Yapom & & $E=-D V p T h$. OUTE & 563.51 \\
\hline & 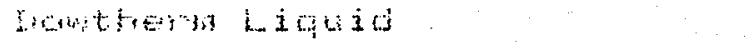 & & EE-DLQTM. OLTE & 568.90 \\
\hline & remeter Gois Inlet & & $E E-C I I N$. IUTE. & $418.9=$ \\
\hline & 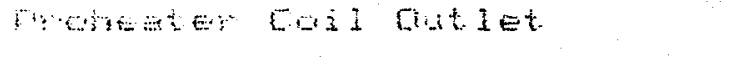 & & $E E-C 1 O H T$. DLITE & 535.31 \\
\hline & S1 irry me-teater Injet & & EE-EGFIM. OUT & 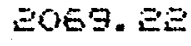 \\
\hline & 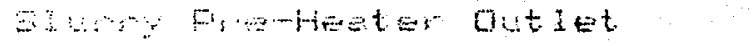 & & E9OtFMes. OUT & $209=.34$ \\
\hline & 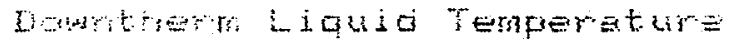 & & E9-DLGT & 633.29 \\
\hline & 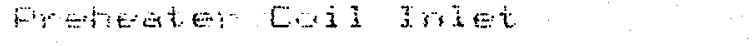 & & ES-CI IM. QLTE & $545 .: 0$ \\
\hline & 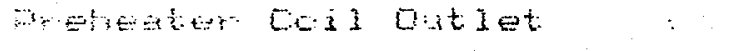 & & E9-C10ut . DLTE & 617.74 \\
\hline 39 & 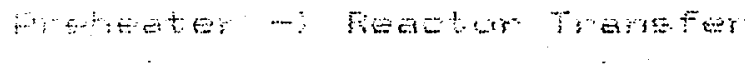 & L_ & $E 9-R 2 x \%$. DUTE & $E=$ \\
\hline & $\because=4 \cdots+\cdots \quad O+1 \leq t$ & & $p s-3 t$ rsap. aUs & $5 \varepsilon 7$. \\
\hline & 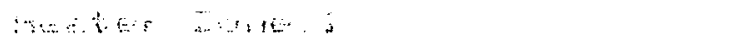 & & FЗ- 3 crete. OUTE & 610.93 \\
\hline & 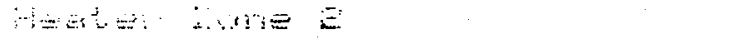 & & $83-20 \cos =013=$ & $013.5 i$ \\
\hline & 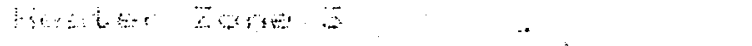 & & Ba-20res. OUT: & 613.49 \\
\hline & 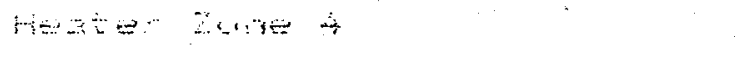 & & 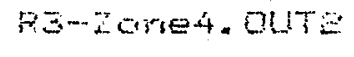 & 609.37 \\
\hline & 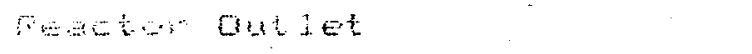 & & RECHThas - OUT & $4 E .30$ \\
\hline & 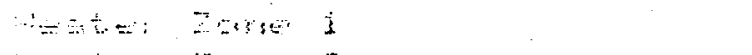 & & $F=-2 \cos i=0 u$ & 31.0 \\
\hline & 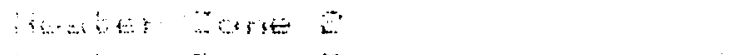 & & $\mathrm{RE}-2 \mathrm{ZnE}$. $\mathrm{CH}$ & 29.6 \\
\hline & $a t a-\infty \quad \therefore a+n=3$ & & Fe-ncres. nut & 35.5 \\
\hline & 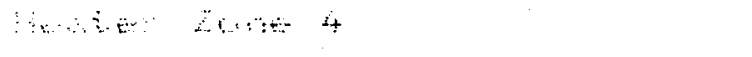 & & 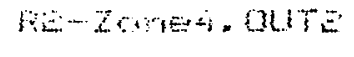 & 03.0 \\
\hline & 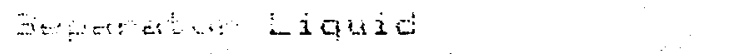 & & ai-1 i & $2+8.39$ \\
\hline & 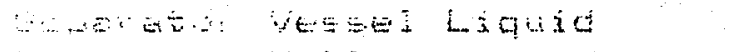 & & S1-IEVE I. GUT & $E 4 \cdot 1 i$ \\
\hline & 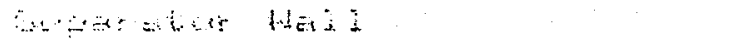 & & $51-6+13.547=$ & $167.4 \%$ \\
\hline & $\therefore$ a & & 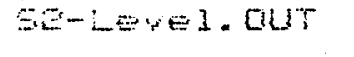 & $=1.30$ \\
\hline as: & $\because a r$ a & & $\theta=-2 \cos =$. Curs & 85.35 \\
\hline & 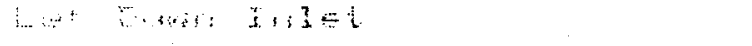 & & 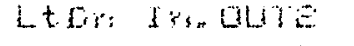 & $549-21$ \\
\hline & i...t $\quad 1 \quad 60 t$ & & Ltbre out. Gute & $E 57.17$ \\
\hline & 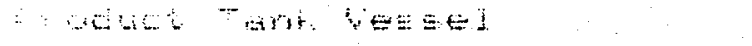 & & FrotTLLVI. OUT & 0.00 \\
\hline & and & & {$[r 10-2=1.01 \mathrm{~T}$} & $39 .: 0$ \\
\hline & $\therefore$ an: & & HWFYH & 2679.6 \\
\hline & 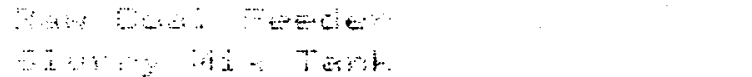 & & 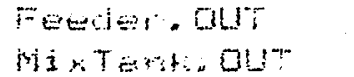 & $\begin{array}{l}200.00 \\
390.70\end{array}$ \\
\hline
\end{tabular}




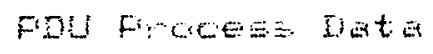

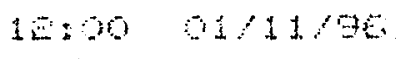

\begin{tabular}{|c|c|}
\hline 5. & 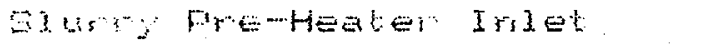 \\
\hline$E 1$ & Powatherm YaFon \\
\hline$E:$ & Dowthen Liquid \\
\hline$E:$ & 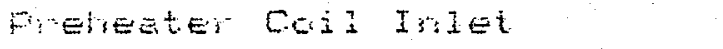 \\
\hline$E i$ & 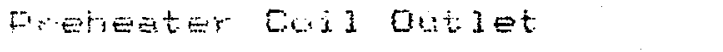 \\
\hline 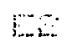 & 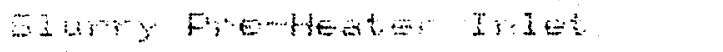 \\
\hline$m$ & 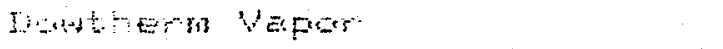 \\
\hline 10 & Dowtots \\
\hline 2 & Fromenter Coil indet \\
\hline$=$ & 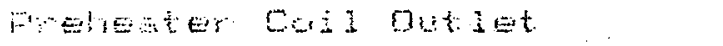 \\
\hline$E=$ & 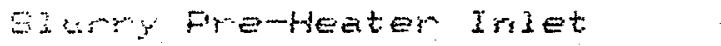 \\
\hline 15 & sumy Fre-beater rut det. \\
\hline$E$ & 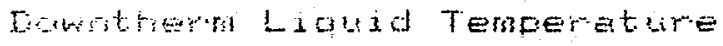 \\
\hline 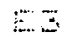 & 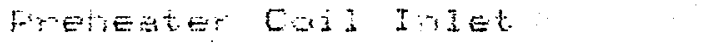 \\
\hline$E \Xi$ & Wethezter [ois Cutiet \\
\hline
\end{tabular}

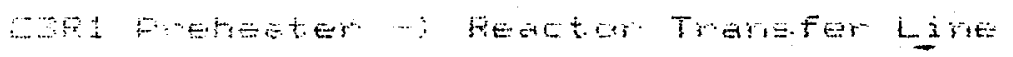

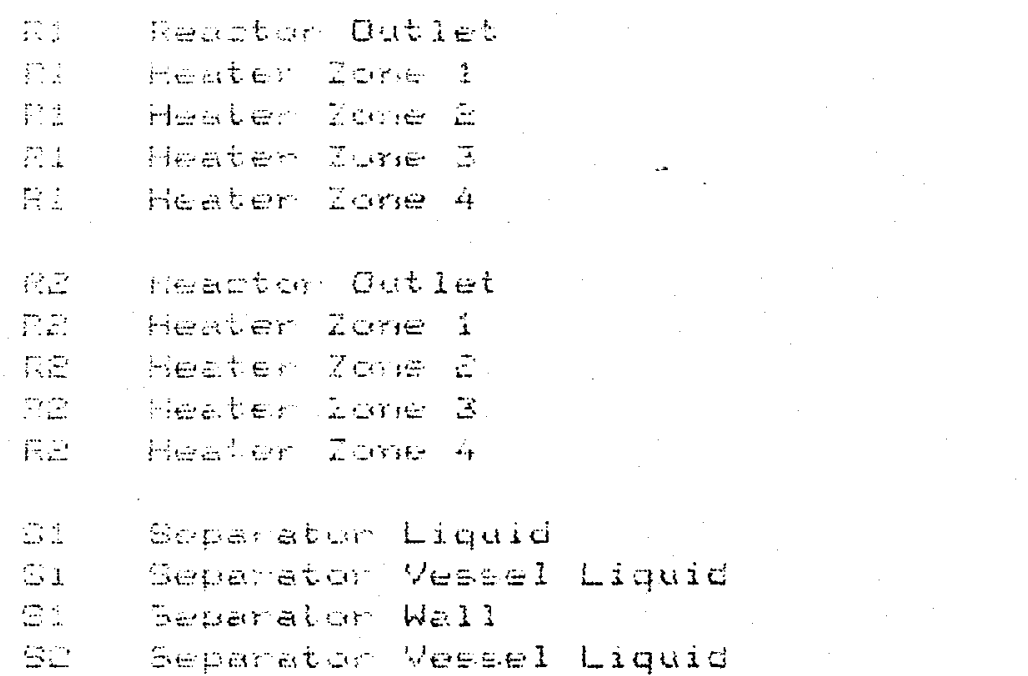

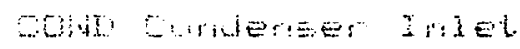

Cat

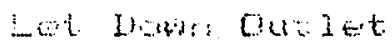

molut Trat Ves.

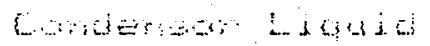

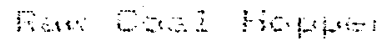

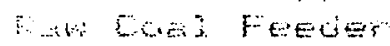

a d a

\begin{tabular}{|c|c|}
\hline EETrFres. Gut & 1EIE. S: \\
\hline E-DUPTII. OLIE & 250.10 \\
\hline S-DLQTH. OUTE & 503.6 \\
\hline$=-C_{1}$ In. OUTL & $19 E .1$ \\
\hline -CI Gut. GILTE & 375. \\
\hline E-ESFYS. OUT & $17 \%$. \\
\hline E-DUPTH. QUTE & EEE. 1 \\
\hline S-DLQTIM. OUTE & EES. \\
\hline E-CI Ir. OUTE & 377.5 \\
\hline E-CIOUt. OUTE & 532 \\
\hline$E-E G P Y S . Q U T$ & E051. \\
\hline E9OtFrES. OUT & $=055.73$ \\
\hline ES-DLQTH. OUTE & $63 \theta . \equiv 5$ \\
\hline EF-CI In. OUTE & 532.20 \\
\hline ES-C10ut. OUTE & E1E. \\
\hline ES-F $3 \times$ XUTE & E. 14. \\
\hline$\because 3-$ - It TrF. OU $\equiv$ & EEE. \\
\hline R-Z-ZTES. OUTE & \#こ. 3 \\
\hline R2-zorez. OUTE & 000.06 \\
\hline 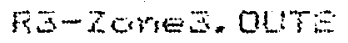 & 603.58 \\
\hline F- & $630.5 \mathrm{E}$ \\
\hline $5=0+7$ & 53 \\
\hline$€ 1.0 \mathrm{cu}$ & $=3$ \\
\hline FE-ZMSEE. QUT & $=0.10$ \\
\hline $5=--$ are $\Xi$. CHUT $=$ & 30 \\
\hline 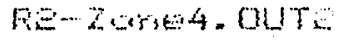 & 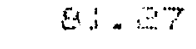 \\
\hline si-tad. ours & $2=1.3$ \\
\hline EI. OUT & 58 \\
\hline $9 \pm-$ wa 12 . OUT & $157 \cdot+\frac{1}{3}$ \\
\hline $9 \mathrm{~s}-L \mathrm{t}=1 . \mathrm{OLT}$ & a. 2 \\
\hline$P 3-2 a r i b \pm$ as & $8=.87$ \\
\hline 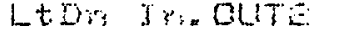 & 539.30 \\
\hline LtDr, out = cuThe & $2=6.69$ \\
\hline 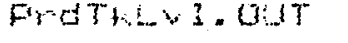 & 0.0 \\
\hline Crad-Lew1. aur & 43.11 \\
\hline HWFE, a OUT & $194.5=$ \\
\hline Feder. aU & $=00.00$ \\
\hline Ma Tart. out & $3=5.5$ \\
\hline
\end{tabular}


Fol Fonges Das

$10=150 \%$

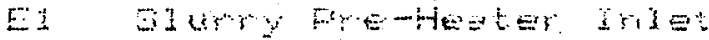

EEITFTES. CUT

$180 \% .60$

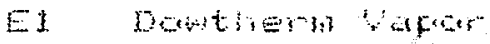

E1 Dowthew in iquid

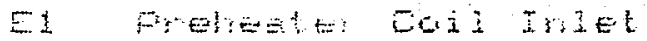

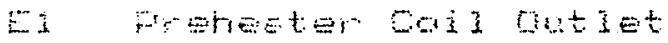

EE-OUPTH. QUTTE

345.37

EE-WLGTH. OUTE

500.14

EE-C1 IN. OUTE

194.23

EE-CIQUT. OLTE

उEE. E

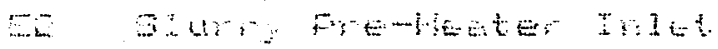

ES-ESFro. OUT

$169+205$

EE-DVPTIA. OLTE

$5=13.5$

Ex-OLGTh. QUTE

$5 E 8 \times 37$

ES-C. I I. OLTE

36. 65

ES-CIOUt. OLTE

519.39

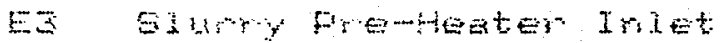

ES-ESTrE. OUT

2033.98

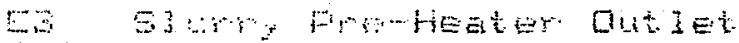

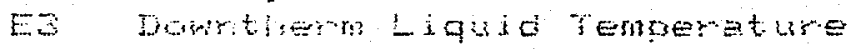

Egatfres. OUT

$\$ 036.03$

ES-DLQTH. OUTE

640.53

ES-EI Im. OUTE

519.15

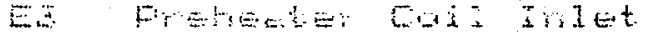

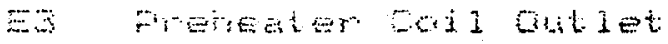

ES-6i0.1. OUTE

633.54

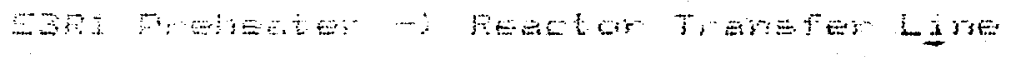

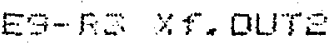

E. 15

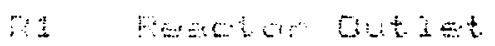

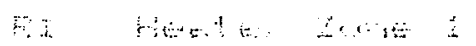

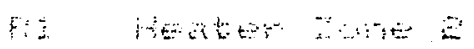

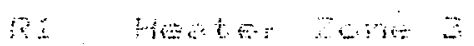

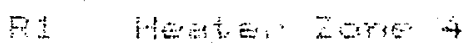

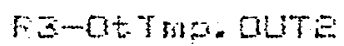

Evi. $7 y$

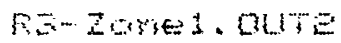

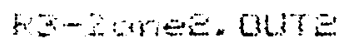

$6 z^{2}-97$

$5=11$

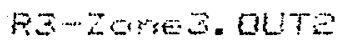

E*1.94

Fo- 2 rat. OLW

$E=4,78$

\begin{tabular}{|c|c|c|}
\hline 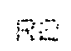 & Sexted & $\operatorname{Gid} 1 \mathrm{~L}$ \\
\hline 2 & 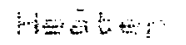 & $\therefore-m \leq 1$ \\
\hline & $\operatorname{tros}$ & $\therefore$ ता \\
\hline & $\sec 6=$ & 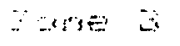 \\
\hline & मo & $\therefore$ are 4 \\
\hline
\end{tabular}

RETE? InF - DUT

47.57

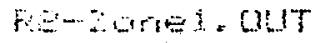

$=9.56$

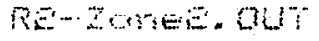

$\Xi E . E \%$

FE-z-rs

74.43

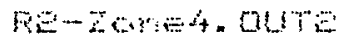

70.6

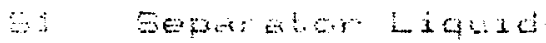 \\ i Sefar a

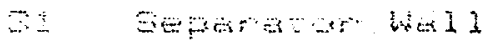 \\ s. 5 -}

$3-39 \mathrm{~d} . \mathrm{Ou}: 2$

21G. ES

Si-LEvEI. OU:

59.6

$53-4613.04 T 2$

159. 4

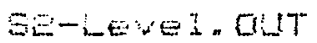

$=1.18$

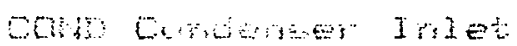

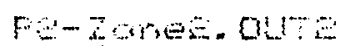

(20.5)

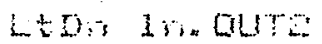

LEDr: CHE. OLITE

B⿺辶一兀

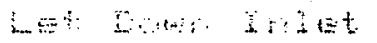

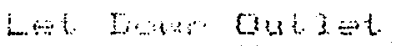

prots Thr Yet

Fith-Y. CUT

EE. E.

a.

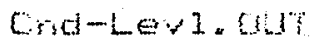

44,19

HOFF: . OLT

780.57

Feeder. OLT

स्राण.

M

$19: 79$ 
Dou Fraceses Date

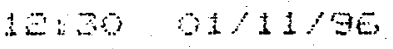

E1 Sumry Fo-Heater libet

EEIrifres. OUT

1795.09

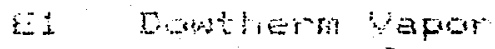

E: Domtierm Liquid

Ei Ferbater Cail Iriset

E. Freteeter Cois outiet

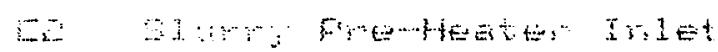

EE-DYPTM. OUTL

301.41

EE-DLGTH. OUTE

500.23

EE-CI Ir. OUTE

191.75

EE-ciaut. QuTE

उEs. 63

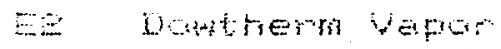

E

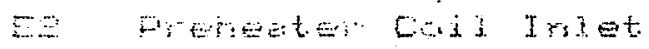

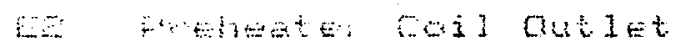

EE-EETHE. QUT

1Eg3.7?

EE-DVPTH. OUTE

EG-DLGTH. OUTE

ES-CI Ir. QUTE

ES-CIDUt. OUTE

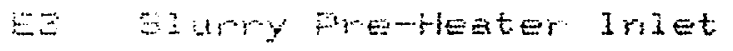

EE-E9FrS. OUT

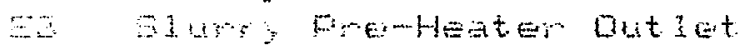

E90tFres. OUT

ES-DLQTH. OUTE

EG-CI IN. OUTE

E9-C10ut. RUTE

553.50

561.04

307. 91

500.35

2013. 79

$20=4 . \pm 9$

6ละ. 92

500.36

606. 54

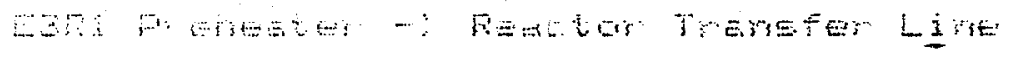

EQ-FZ YF. OUTE

608.40

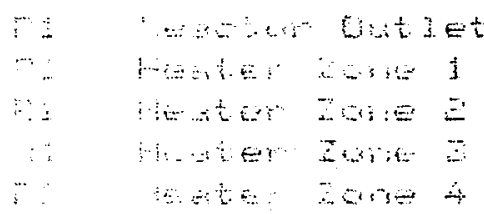

R-Ch Tmp. Qute

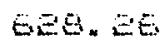

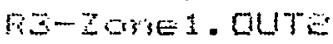

อ15. $1:$

Fu-zorez. GuT2

EEs. 34

RE-Zorses. OUTE

BEE. 74

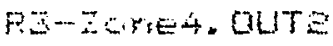

631.14

REQTTMF. OUT

40.13

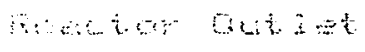

PE-zastei. QuT

27.94

RE-zGret. OUT

ㄹ. 41

Mate

trats ante 3

F E EMES. OLTTE

$7 \% .5 E$

ne-zares. GLTE

76.2

61-i.iag. OLTE

199.44

Ei-LEvei. aUT

54.44

Si-Hail. OUT

IEE. 63

SE-Levei. CuT

E1. 10

PQ-ZGren. QUTE

79.49

itor Ir. oure

Lt.me bit. OUTE

41.60

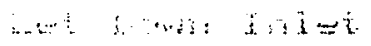

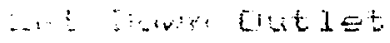

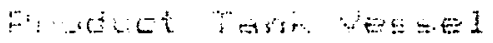

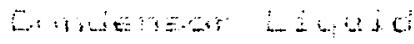

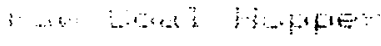

FrdTBLova OUT

249. 64

. $0 \%$

Cro-1-E. . DUT

40.82

Howper. OUT

FEeder. OLT

$1624,3 \%$

20.60

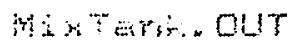

$20 \% \cdot 23$ 
Wou Fraceses Data

उट:45 0.11/96

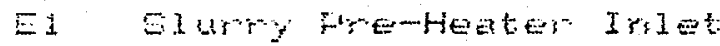

EEIMFMES. OUT

EE-DVPTM. QUTE

EE-DLGTH. QUTTE

EG-CI Ir. QUTE

EE-CIOUT. OUTE

EE-ESFrE. OUT

ES-DVPTH. OUTE

EE-DLGTH. OUTE

EE-CI Ir. OUTE

EE-CIOUT. OUTE

EE-E9Frs. OUT

ESOtFres. QUT

E9-DLGSH. OUTE

EG-CI Ir. QUTE

ES-CIOut. QUTE

ES-FS XF. OUTE

PD-OtTme. QUTE

52-zari- DUTE

PE-zorea. OUT:

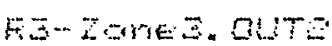

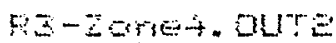

PEDtrma. OUT

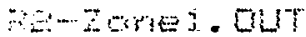

RE I OMES. OUT

Pe-Zorim 3 . dLT:

RE-ZGrES. OUTE

3j-Ligo. GLTE

St-Levei. OUT

EI-Wail. OLTS

BE-LEVES. OUT

Pe-rones oun

Lton In. OLT

L.tin aut oute

FrothL' 1. OUT

Cro-lew1. our

Hagne? "OUT

Feeder. DUT

ins $x$ Tart. OUT i 790.33

$35 \mathrm{~s} \cdot 45$

501.28

196.10

369. 15

1699.91

$55 x^{2} \cdot 34$

$56 \Xi .26$

309.56

517.55

$20=0.34$

2030.44

ESE. 71

515. 85

EOE. 98

EDe. 94

E. 2.00

E14.55

52=.09

$6=4.90$

EET.

36. 4

27.75

ㄹ․ 13

77.63

70 ब

194.15

5 ㄷ․ 7

160.64

$\pm 1.20$

790 209.7

303.98

0.00

40.5

12.3. 19

Eo. 00

24. 
Ful Foc

$13: 00$ of 1900

\begin{tabular}{|c|c|}
\hline 돈 & SI uny Frableder Iritet \\
\hline$E 2$ & Dont? \\
\hline$\because 1$ & Donttrenta Liquid \\
\hline$E 1$ & Fond \\
\hline$E j$ & 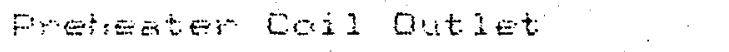 \\
\hline$E$ & 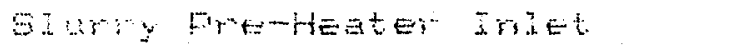 \\
\hline 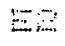 & 1) \\
\hline$=$ & 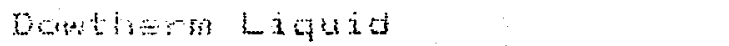 \\
\hline & 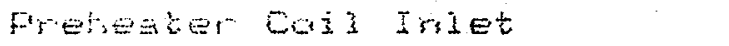 \\
\hline$i=$ & 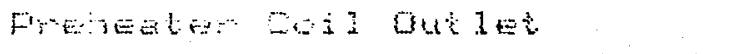 \\
\hline & 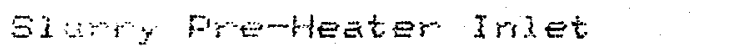 \\
\hline & 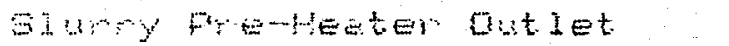 \\
\hline & Downthend liguid temperature \\
\hline & 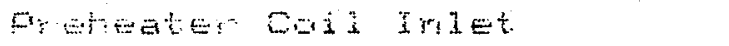 \\
\hline & 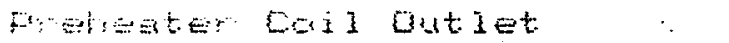 \\
\hline
\end{tabular}

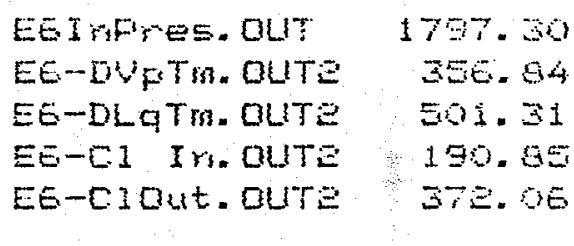

EE-ESFT. OUT

EE-DUPTM. OLTE

ES-DLgTm. QUT:

EE-CI 1 r. OUT:

EE-CIOut. QUTE

ES-EGFHS. CUT

EgOtFres. OUT

E9-DLGTH. OUTE

ES-CI IM. OUTE

E9-C:1 Qut. CUTE

ES-R3 $x+.04 T=$

F- $3-0$ t THF- QUTE

RE-ZCW

FE- EArsE OUTE

PS-ZCHES. OLT:

Fa- are4. OLTE

REOT FHF OUT

$R=-201=1$. QUT

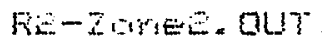

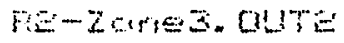

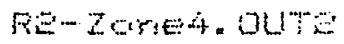

3i-Liqd. QLTE

31-i_eneI. dur

31-Ha11. QUTE

SE-LEWE I . OUT

FE-2arsen OLITE

LEN IN OHTE

Ltrim Gut. GLTE

FrodThaw . OUT

Cred-:Ev. OUT

HOPPE: OUT

FEEder, QUT

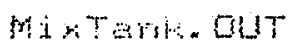

$109=.00$

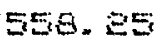

.564. Es

371.96

519.6E

$20=0.48$

2031.13

63‥43

도. 35

603.93

644.65

53.

$E 14$. 13

E. 0.50

으. 3.

E으.

$53 . E$

$=7.43$

$=5.94$

76.31

$77 \times 30$

163.53

49.7

$1=4.70$

E. 43

76.51

610.66

퀄.

5.00

35.91

530.09

ज्ञात 0

$+4.73$ 
Fou procese Deta

is:15 01filo

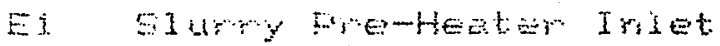

EE inses. OUT

$1784=610$

Ei Dowbriem Yapor

Ei Dowthern Liquid

Es Frefeater Coil InIEt

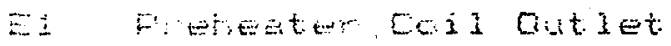

EE-DVFTH. QUT:

36:- 53

EE-DLGTM. OUTE

5013

EE-CI Ir. QUTE

Es3.

Es-EIOUt. OUTE

$403.5 E$

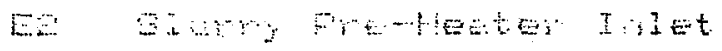

EE-EEPr-D. OUT

1679.09

EE-DUpTr. OUTE

$54 \mathrm{E} .01$

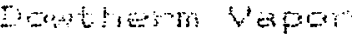

EE Fewthem Ligum

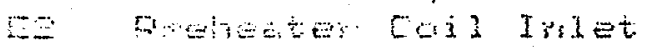

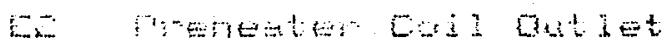

ES-DLGTH. OUTE

EE-CI IYI OUTE

ES-CIOUT. QUTE

돈.TE

395.50

515.44

ES Slurry me-Heater Inlet

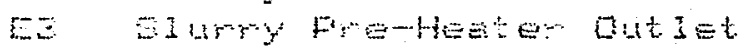

ES-EGFirs. OUT

5006. 93

Egot Fres. OUT

9016.99

ES-DLGTH. QUTE

641.15

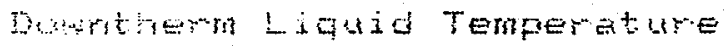

E9-C. I IN. QUTE

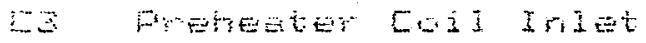

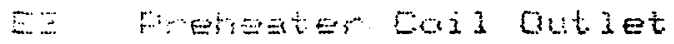

E9-010ut. QUTE

517.85

510. $9 E$

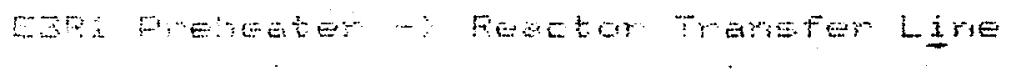

ES-RS XF. OUTE

610.99

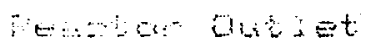

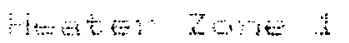

Heater zore $=$

Hoster zerit

W-Et Tha. OUTS

PS-Zarie1. QUTE

Ri-zoriez. QUTE

R3-Zores. CUTE

D-zome4. OUTE

5 E. 94

6.:. $5: 5$

627.33

500.59

EEE. 7

ReQtTMF. OUT

21.00

PE-zorses. Glt

RE-ZCOHE. DUT

20. 69

2.9. 48

FE-zorez. OLTE

70.9

ME-ZCoreis. DUTE

70.0

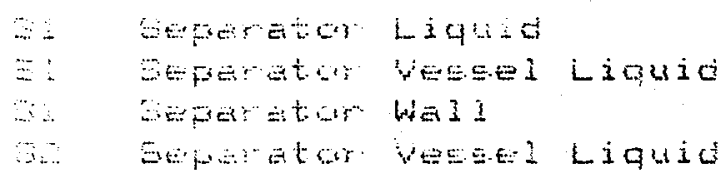

gij-iqd. aute

91-i-eveI. OUT

S1-Wais oUTe

GE-LEVI. OLT

I7E. 45

49.56

149.55

… 31

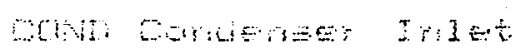

PE-ZMED. OUTE

77.85

Ltorr Ir. OUTE

LtDr out. OUTE

0.1 .69

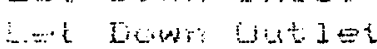

Frobed Tar Vessei

Conterson - - iquid

FrUTKLYI. OLIT

243.68

0.00

Crod-ievi. duT

42.03

Hopper. OUT

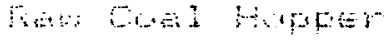

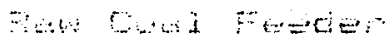

Feeder. DUT

i. 6

his Tork. OUT

ह0.

a. 5.5 
pou prosed Data

$12: 30$, $11 / 96$

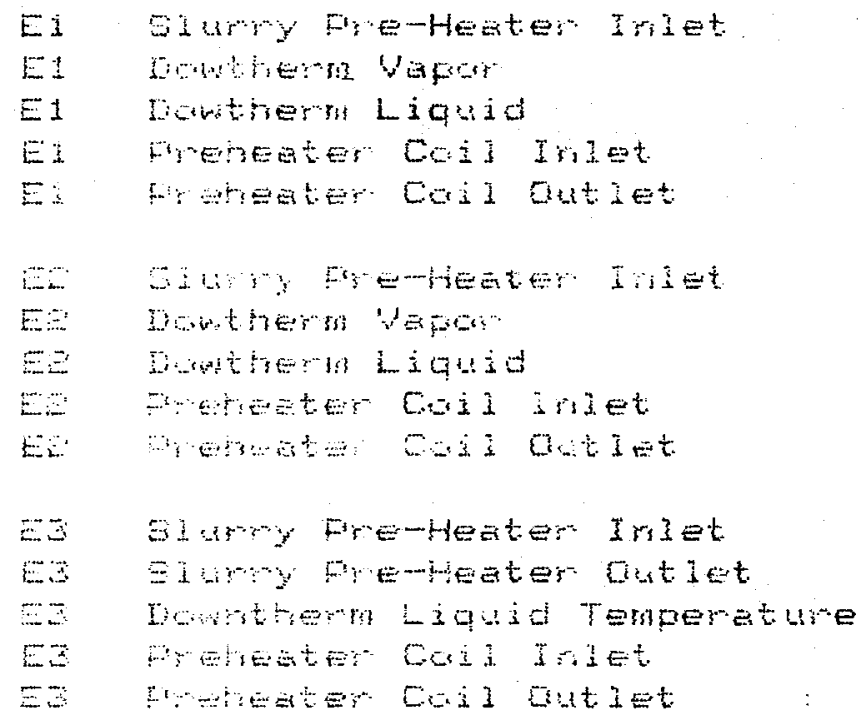

EEIrFfres. OUT

$18 \div 0.8 E$

EE-DUFTR. OUTE

401.19

EE-DLQTH. OUTE

EG-C1 Ir. QUTE

EE-Cidut. OUTE

EIi. 0

อ7Е. 90

457.05

EE-ESFT: OUT

1708.45 EE-DUPTH. OUTE ES-DLqTH. OUTE EE-CI In. oute E5-C10ut. QuTE

581.08 58‥ .98 435.08

58s. 99

EE-EGPrS. OUT

E9Qt.Fres. OUT

0053.40

0060.05 E9-DLGTH. OUTE

633.85 EY-C.3 Ir. OUTE ES-Cicut. QUTE

563.55

613. 5

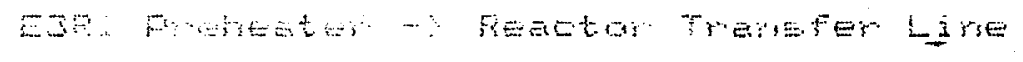

EO-RA Xf. OUTE

614.56

F.-Dt ThP. OLTE

230.93

PE-IGNe 1. OUTE

n3-2orese OUTE

RS-ZGres. OUTE

F3-zorie4. OLire

61..20

$6 \mathrm{EO} .6 \mathrm{E}$

6อ9. 09

535.45

REOTrma. OUT

30.5

FE-Zares . OLT

ㄹ․ 71

Re-Zarite. OUT

25.

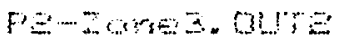

75.70

Pe-zires. OUT:

70.9

51-1-iact. OUTE

191.25

49.85

Si-ieves. OUT

si-bali. oure

SE-LEvi. ouT

146.78

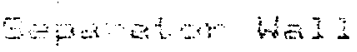

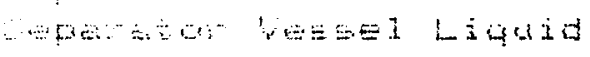

ㄹ. 44

PE-rorea OHTE

77.49

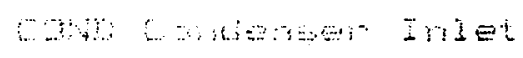

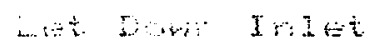

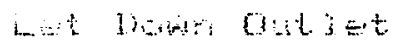

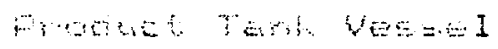

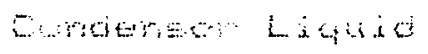

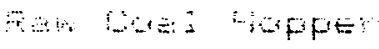

Mar Cod Fereder

…

LtDm Ts. ours

Ltom Oat QuTs

00.26

ES. $5 E$

Frorkini. ouT

Crid-i.evl. QLi?

0.60

Hepker. our

Feeder. our

ivi i T Tark. OUT

45.03

2.60

179.49

$4: 45$ 
Energy \&

Environmental Research

Center

APPENDIX E

HWD PROCESS GAS AND WATER ANALYSIS 


\section{Energy \&}

Environmental

Research

Center

Run \# THAI

Date: $\quad 11-13-95$

$\begin{array}{lrrr} & \text { THAI.01 } & \text { THAl.03 } & \text { THAl.06 } \\ \text { Sample: } & 300 \mathrm{C} & 325 \mathrm{C} & 275 \mathrm{C}\end{array}$

Helium

0.06

0.33

43.25

Carbon Dioxide

Propane

Propylene

Acetylene

iso-Butane

Carbonyl Sulfide

n-Butane

Hydrogen Sulfide

1-Butene

iso-Butylene

t-2-Butene

iso-Pentane

c-2-Butene

$n$-Pentane

1,3-Butadiene

Ethylene

Ethane

Oxygen/Argon

Nitrogen

Methane

Carbon Monoxide

Calc. BTU/SCF

$$
\text { Sat. }
$$

Dry

16.3

16.5

0.05

0.06

0.69

53.31

0.76

1.30

0.02

0.02

0.17

0.27

0.31

0.02

0.06

0.05

0.12

0.11

0.23

0.20

1.80

1.74

10.60

1.90

10.50

2.30

1.60

2.25

42.9

42.9

43.7

43.7

Calc. Sp. Gravity

1.202

Calc. Ave. Mol Wt.

34.79

1.413

1.420

40.82

41.00 
Energy \&

Environmental

Research

Center

Gas Analysis Report

Process Chemistry \& Development Laboratory

Run \# THAILAND

Date: $\quad 11-9-95$

\section{THAl.06}

\section{Mole Per Cent}

Sample: $275 C$ Repeat

Helium

0.02

Hydrogen

0.53

Carbon Dioxide

82.86

Propane

Propylene

0.43

Acetylene

iso-Butane

Carbonyl Sulfide

$n$-Butane

0.02

Hydrogen Sulfide

0.30

1-Butene

iso-Butylene

0.05

t-2-Butene

iso-Pentane

c-2-Butene

$n$-Pentane

1,3-Butadiene

Ethylene

0.11

Ethane

0.20

Oxygen/Argon

1.56

Nitrogen

10.28

Methane

1.58

Carbon Monoxide

2.06

Calc. BTU/SCF

$\begin{array}{ll}\text { Sat. } & 43.2 \\ \text { Dry } & 43.9\end{array}$

Calc. Sp. Gravity

Calc. Ave. Mol Wt. 


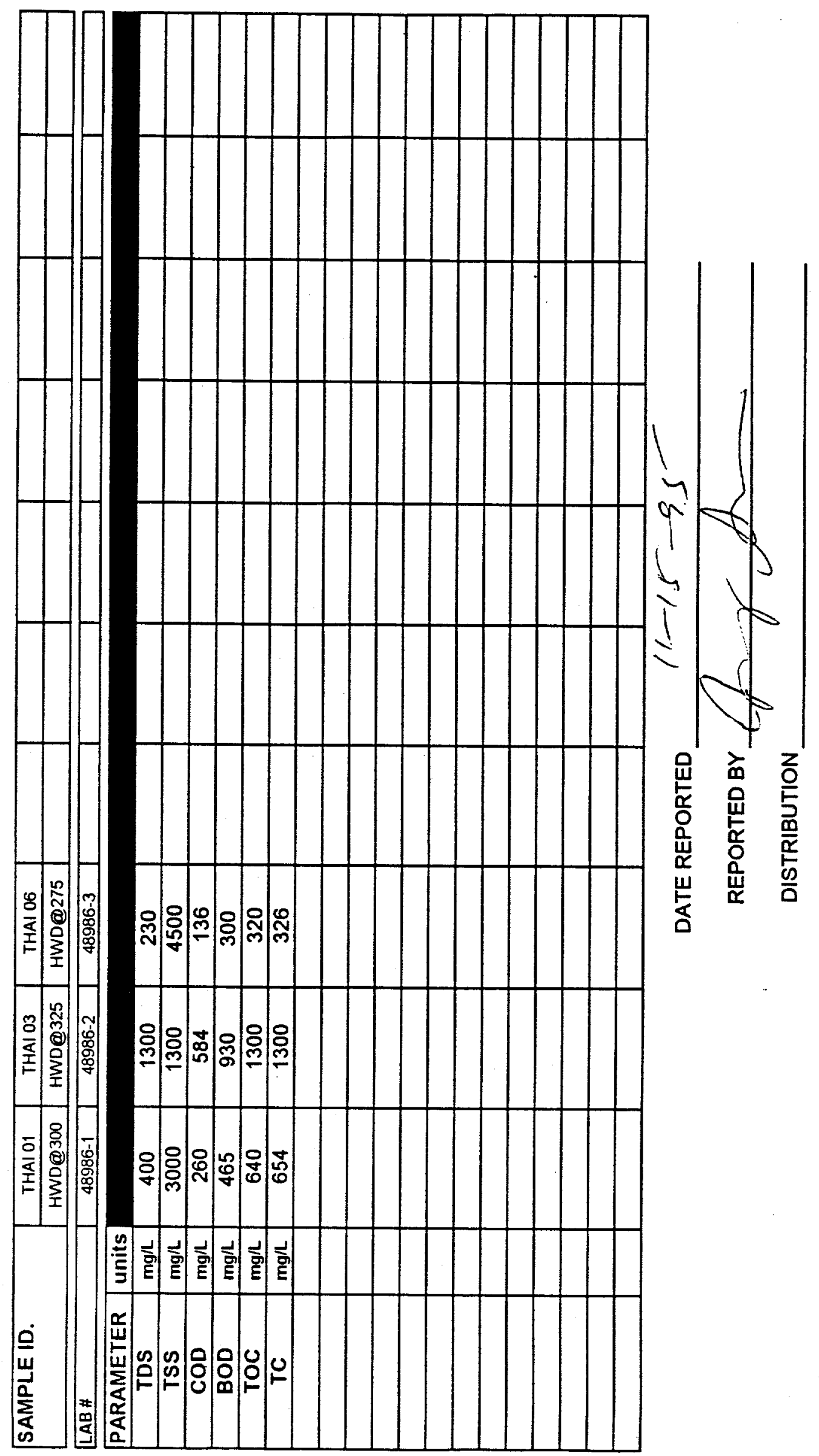


Energy \&

Environmental

Research

Center

APPENDIX F

GASIFICATION TEST M538 


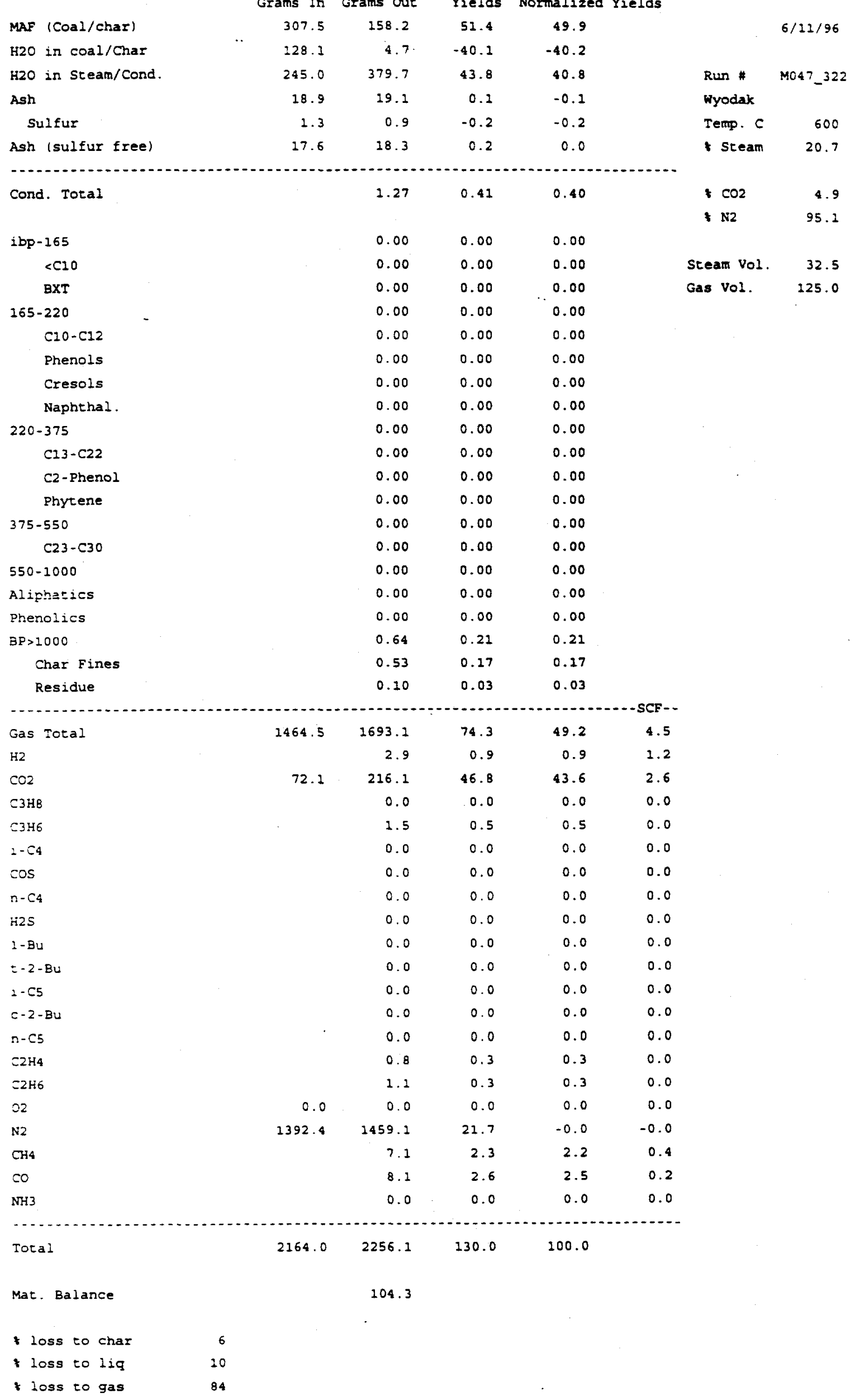




\begin{tabular}{|c|c|c|c|c|c|c|c|}
\hline $\begin{array}{l}\text { Feed In/Residuals Out } \\
\mathrm{H} 2 \mathrm{O} \text { in Feed/Residual } \\
\mathrm{H} 2 \mathrm{O} \text { in } \mathrm{H} 2 \mathrm{O} / \text { Cond. } \\
\text { Ash }\end{array}$ & $\begin{array}{r}M \\
\text { Gms In } \\
2047.5 \\
811.4 \\
3002.0 \\
124.1\end{array}$ & $\begin{array}{r}\text { laterial B } \\
\text { Gms Out } \\
431.3 \\
11.3 \\
3431.0 \\
36.3\end{array}$ & $\begin{array}{c}\text { lance S } \\
\text { Yields } \\
21.1 \\
-39.1 \\
21.0 \\
-4.3\end{array}$ & $\begin{array}{r}\text { heet Sum } \\
\text { N Yields } \\
85.9 \\
-38.7 \\
21.0 \\
0.9\end{array}$ & $\begin{array}{l}\text { nary } \\
\text { Run \# } \\
\text { Thailand L } \\
\text { Temp } \\
\text { Pressure } \\
\% \mathrm{H} 2 \mathrm{O} \\
\% \mathrm{~N} 2 \\
\mathrm{H} 2 \mathrm{O} \\
\text { g Feed }\end{array}$ & $\begin{array}{l}\text { 3-12-96 } \\
\text { M538 } \\
\text { Lignite } \\
699.7 \text { C } \\
29.7 \text { psig } \\
29.9 \\
70.1 \\
\\
1.86\end{array}$ & . \\
\hline $\begin{array}{l}\text { Gas Total } \\
\mathrm{H} 2 \\
\mathrm{CO} 2 \\
\mathrm{C} 3 \mathrm{H} 8 \\
\mathrm{C} 3 \mathrm{H} 6 \\
\mathrm{C} 2 \mathrm{H} 2 \\
\mathrm{i}-\mathrm{C} 4 \mathrm{H} 10 \\
\mathrm{COS} \\
\mathrm{n}-\mathrm{C} 4 \mathrm{H} 10 \\
\mathrm{H} 2 \mathrm{~S} \\
1-\mathrm{C} 4 \mathrm{H} 8 \\
\mathrm{i}-\mathrm{C} 4 \mathrm{H} 8 \\
\mathrm{t}-2-\mathrm{C} 4 \mathrm{H} 8 \\
\mathrm{i}-\mathrm{C} 5 \mathrm{H} 12 \\
\mathrm{c}-2-\mathrm{C} 4 \mathrm{H} 8 \\
\mathrm{n}-\mathrm{C} 5 \mathrm{H} 12 \\
1,3-\mathrm{C} 4 \mathrm{H} 6 \\
\mathrm{C} 2 \mathrm{H} 4 \\
\mathrm{C} 2 \mathrm{H} 6 \\
\mathrm{O} 2 \\
\mathrm{~N} 2 \\
\mathrm{CH} 4 \\
\mathrm{CO} \\
\mathrm{NH} 3\end{array}$ & 10951.1 & $\begin{array}{r}10354.4 \\
40.3 \\
384.0 \\
0.0 \\
11.4 \\
0.0 \\
0.0 \\
0.0 \\
0.0 \\
5.9 \\
0.0 \\
0.0 \\
0.0 \\
0.0 \\
0.0 \\
0.0 \\
0.0 \\
14.6 \\
5.8 \\
0.0 \\
9786.0 \\
68.6 \\
37.8 \\
0.0\end{array}$ & $\begin{array}{r}-29.1 \\
2.0 \\
18.8 \\
0.0 \\
0.6 \\
0.0 \\
0.0 \\
0.0 \\
0.0 \\
0.3 \\
0.0 \\
0.0 \\
0.0 \\
0.0 \\
0.0 \\
0.0 \\
0.0 \\
0.7 \\
0.3 \\
0.0 \\
-56.9 \\
3.3 \\
1.8 \\
0.0\end{array}$ & $\begin{array}{r}30.9 \\
2.2 \\
21.0 \\
0.0 \\
0.6 \\
0.0 \\
0.0 \\
0.0 \\
0.0 \\
0.3 \\
0.0 \\
0.0 \\
0.0 \\
0.0 \\
0.0 \\
0.0 \\
0.0 \\
0.8 \\
0.3 \\
0.0 \\
-0.2 \\
3.7 \\
2.1 \\
0.0 \\
\end{array}$ & $\begin{array}{r}\text { SCF } \\
34.4 \\
19.5 \\
8.4 \\
0.0 \\
0.3 \\
0.0 \\
0.0 \\
0.0 \\
0.0 \\
0.2 \\
0.0 \\
0.0 \\
0.0 \\
0.0 \\
0.0 \\
0.0 \\
0.0 \\
0.5 \\
0.2 \\
0.0 \\
-0.1 \\
4.1 \\
1.3 \\
0.0\end{array}$ & & \\
\hline $\begin{array}{l}\text { Total } \\
\text { Mat. Balance } \\
\% \text { Cyclone } \\
\% \text { Main Char Leg } \\
\% \text { loss to liq } \\
\% \text { loss to gas }\end{array}$ & $\begin{array}{r}0 \\
54 \\
0 \\
46\end{array}$ & $\begin{array}{r}14264.3 \\
84.2\end{array}$ & -30.5 & 100.0 & & & $\begin{array}{r} \\
\\
\\
06 / 12 / 96 \\
08: 14\end{array}$ \\
\hline
\end{tabular}




\begin{tabular}{|c|c|c|}
\hline Material Balance Data & & \\
\hline Run Number & M538 Date & $3-12-96$ \\
\hline Fluidization Velocity & $0.72 \mathrm{ft} / \mathrm{s}$ & \\
\hline Run Temperature & $699.7 \mathrm{C}$ & \\
\hline Run Pressure & 29.7 psig & \\
\hline Total N2 & $10951.1 \mathrm{~g}$ & \\
\hline Total Liquid In & $3002.0 \mathrm{~g}$ & \\
\hline Feed Rate & $1.0 \mathrm{~kg} / \mathrm{hr}$ & \\
\hline Run Duration & $3.0 \mathrm{hr}$ & \\
\hline Initial Tank Pressure & 0.0 psig & \\
\hline Final Tank Pressure & 0.0 psig & \\
\hline Final Product Gas Meter Reading & 322.1 SCFH & \\
\hline Mass of Liquid in Traps & $3431.0 \mathrm{~g}$ & \\
\hline Mass of Cyclone Char & $87.0 \mathrm{~g}$ & \\
\hline Mass of Bottom Char & $385.0 \mathrm{~g}$ & \\
\hline Defaults: & & \\
\hline Temperature at Gas Meter & $15.56 \mathrm{C}$ & \\
\hline Pressure at Gas Meter & 0.0 psig & \\
\hline
\end{tabular}




\begin{tabular}{|lrr|}
\hline Char Analysis & Run \# & M538 \\
& \multicolumn{2}{c|}{ As Recvd } \\
& Bottom & Cyclone \\
& & \\
Proximate Analysis (\%) & 0.60 & 2.40 \\
Moisture & 15.11 & 18.31 \\
Volatile Matter & 76.89 & 70.27 \\
Fixed Carbon & 7.40 & 9.02 \\
Ash & 100.00 & 100.00 \\
& & \\
Ultimate Analysis (\%) & 0.00 & 0.00 \\
Hydrogen & 0.00 & 0.00 \\
Carbon & 0.00 & 0.00 \\
Nitrogen & 0.00 & 0.00 \\
Sulfur & 0.00 & 0.00 \\
Oxygen (Diff) & 0.00 & 0.00 \\
Sulfur & 0.00 & 0.00 \\
& & \\
Sulfur (\%) & 0.00 & 0.00 \\
Organic Chlorine & 0.00 & 0.00 \\
Inorganic Chlorine &
\end{tabular}




\begin{tabular}{|c|c|c|c|c|c|c|}
\hline & & & Run \# & M538 & & \multirow{3}{*}{ Average } \\
\hline \multicolumn{2}{|c|}{ Air Free Analysis } & \multicolumn{2}{|c|}{$\begin{array}{l}\text { Yes } \\
\text { Gas Analysis } \\
\text { Gas Bag } 1 \text { Gas Bag } 2\end{array}$} & \multicolumn{2}{|c|}{$\begin{array}{l}\text { AIRJHELIUM FREE } \\
\text { Gas Bag } 1 \text { Gas Bag } 2\end{array}$} & \\
\hline $\mathrm{He}$ & Helium & 0.02 & 0.03 & & & \\
\hline H2 & Hydrogen & 5.13 & 5.31 & 5.14 & 5.32 & 5.23 \\
\hline $\mathrm{CO} 2$ & Carbon Dioxide & 2.19 & 2.33 & 2.20 & 2.34 & 2.27 \\
\hline $\mathrm{C} 3 \mathrm{H} 8$ & Propane: & & & 0.00 & 0.00 & 0.00 \\
\hline $\mathrm{C} 3 \mathrm{H} 6$ & Propylene & 0.06 & 0.08 & 0.06 & 0.08 & 0.07 \\
\hline $\mathrm{C}_{2} \mathrm{H}_{2}$ & Acetylene & & & 0.00 & 0.00 & 0.00 \\
\hline $\mathrm{i}-\mathrm{C} 4 \mathrm{H} 10$ & iso-Butane & & & 0.00 & 0.00 & 0.00 \\
\hline $\cos$ & Carbonyl Sulfide & & & 0.00 & 0.001 & 0.00 \\
\hline n-C4H10. & n-Butane & & & 0.00 & 0.00 & 0.00 \\
\hline H2S & Hydrogen Sulfide & 0.05 & 0.04 & 0.05 & 0.04 & 0.05 \\
\hline $1-\mathrm{C} 4 \mathrm{H8}$ & 1-Butene & & & 0.00 & 0.00 & 0.00 \\
\hline $\mathrm{i}-\mathrm{C} 4 \mathrm{H} 8$ & iso-Butylene & & & 0.00 & 0.00 & 0.00 \\
\hline $\mathrm{t}-2-\mathrm{C} 4 \mathrm{H} 8$ & t-2-Butene & & & 0.00 & 0.00 & 0.00 \\
\hline $\mathrm{i}-\mathrm{C} 5 \mathrm{H} 12$ & iso-Pentane & & & 0.00 & 0.00 & 0.00 \\
\hline$c-2-C 4 H 8$ & c-2-Butene & & & 0.00 & 0.00 & 0.00 \\
\hline $\mathrm{n}-\mathrm{C} 5 \mathrm{H} 12$ & n-Pentane & & & 0.00 & 0.00 & 0.00 \\
\hline 1,3-C4H6 & 1,3-Butadiene & & & 0.00 & 0.00 & 0.00 \\
\hline $\mathrm{C} 2 \mathrm{H} 4$ & Ethylene & 0.13 & 0.14 & 0.13 & 0.14 & 0.14 \\
\hline $\mathrm{C} 2 \mathrm{H} 6$ & Ethane & 0.05 & 0.05 & 0.05 & 0.05 & 0.05 \\
\hline $\mathrm{O} 2$ & Oxygen & 0.05 & 0.04 & & & 0.00 \\
\hline N2 & Nitrogen & 90.93 & 90.44 & 90.98 & 90.50 & 90.74 \\
\hline $\mathrm{CH} 4$ & Methane & 1.05 & 1.17 & 1.05 & 1.17 & 1.11 \\
\hline $\mathrm{CO}$ & Carbon Monoxide & 0.34 & 0.36 & 0.34 & 0.36 & 0.35 \\
\hline NH3 & Ammonia & & & 0.00 & 0.00 & 0.00 \\
\hline \multicolumn{7}{|l|}{ Total } \\
\hline \multicolumn{2}{|c|}{ Avg. Mole Wt } & 26.9002 & 26.8580 & & & 26.8820 \\
\hline
\end{tabular}


Energy \&

Environmental

Research

Center

\section{APPENDIX G}

\section{GASIFICATION TEST M539}




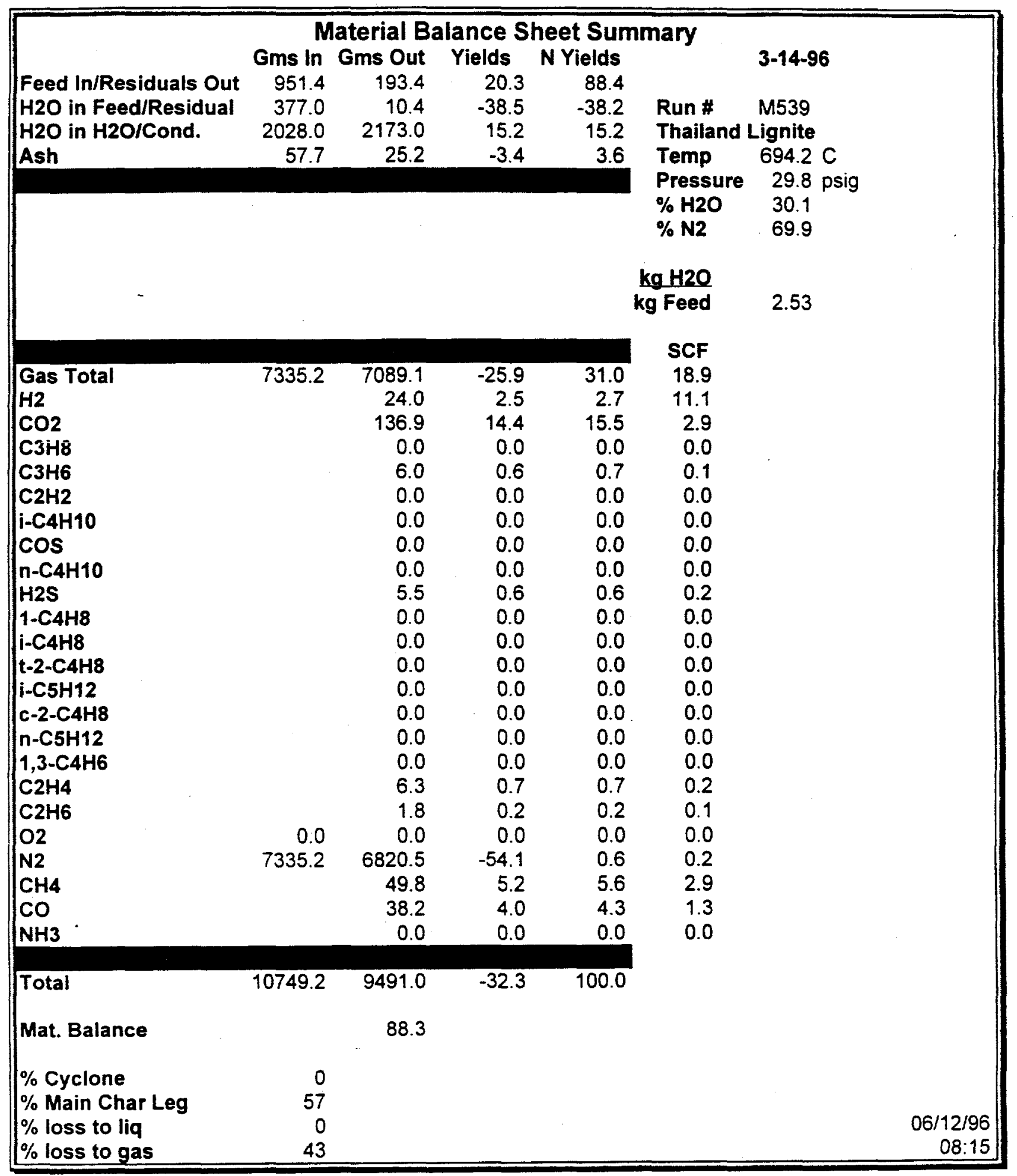




\begin{tabular}{|lc||}
\hline Material Balance Data & \\
Run Number & M539 Date $\quad 3-14-96$ \\
Fluidization Velocity & $0.72 \mathrm{ft} / \mathbf{s}$ \\
Run Temperature & $694.2 \mathrm{C}$ \\
Run Pressure & $29.8 \mathrm{psig}$ \\
Total N2 & $7335.2 \mathrm{~g}$ \\
Total Liquid In & $2028.0 \mathrm{~g}$ \\
Feed Rate & $0.7 \mathrm{~kg} / \mathrm{hr}$ \\
Run Duration & $2.0 \mathrm{hr}$ \\
Initial Tank Pressure & $0.0 \mathrm{psig}$ \\
Final Tank Pressure & $0.0 \mathrm{psig}$ \\
Final Product Gas Meter Reading & $220.6 \mathrm{SCFH}$ \\
Mass of Liquid in Traps & $2173.0 \mathrm{~g}$ \\
Mass of Cyclone Char & $132.0 \mathrm{~g}$ \\
Mass of Bottom Char & $93.0 \mathrm{~g}$ \\
& \\
Defaults: & $15.56 \mathrm{C}$ \\
Temperature at Gas Meter & $0.0 \mathrm{psig}$ \\
\hline
\end{tabular}




\begin{tabular}{|lrr|}
\hline Char Analysis & Run \# & M539 \\
& \multicolumn{2}{c|}{ As Recvd } \\
& Bottom & Cyclone \\
Proximate Analysis (\%) & & \\
Moisture & 0.40 & 4.60 \\
Volatile Matter & 10.11 & 17.61 \\
Fixed Carbon & 79.86 & 65.31 \\
Ash & 9.36 & 12.49 \\
& 99.73 & 100.01 \\
Ultimate Analysis (\%) & & \\
Hydrogen & 0.00 & 0.00 \\
Carbon & 0.00 & 0.00 \\
Nitrogen & 0.00 & 0.00 \\
Sulfur & 0.00 & 0.00 \\
Oxygen (Diff) & 0.00 & 0.00 \\
Sulfur & 0.00 & 0.00 \\
& 0.00 & 0.00 \\
Sulfur (\%) & & $\cdot$ \\
Organic Chlorine & 0.00 & 0.00 \\
Inorganic Chlorine & 0.00 & 0.00 \\
\hline
\end{tabular}




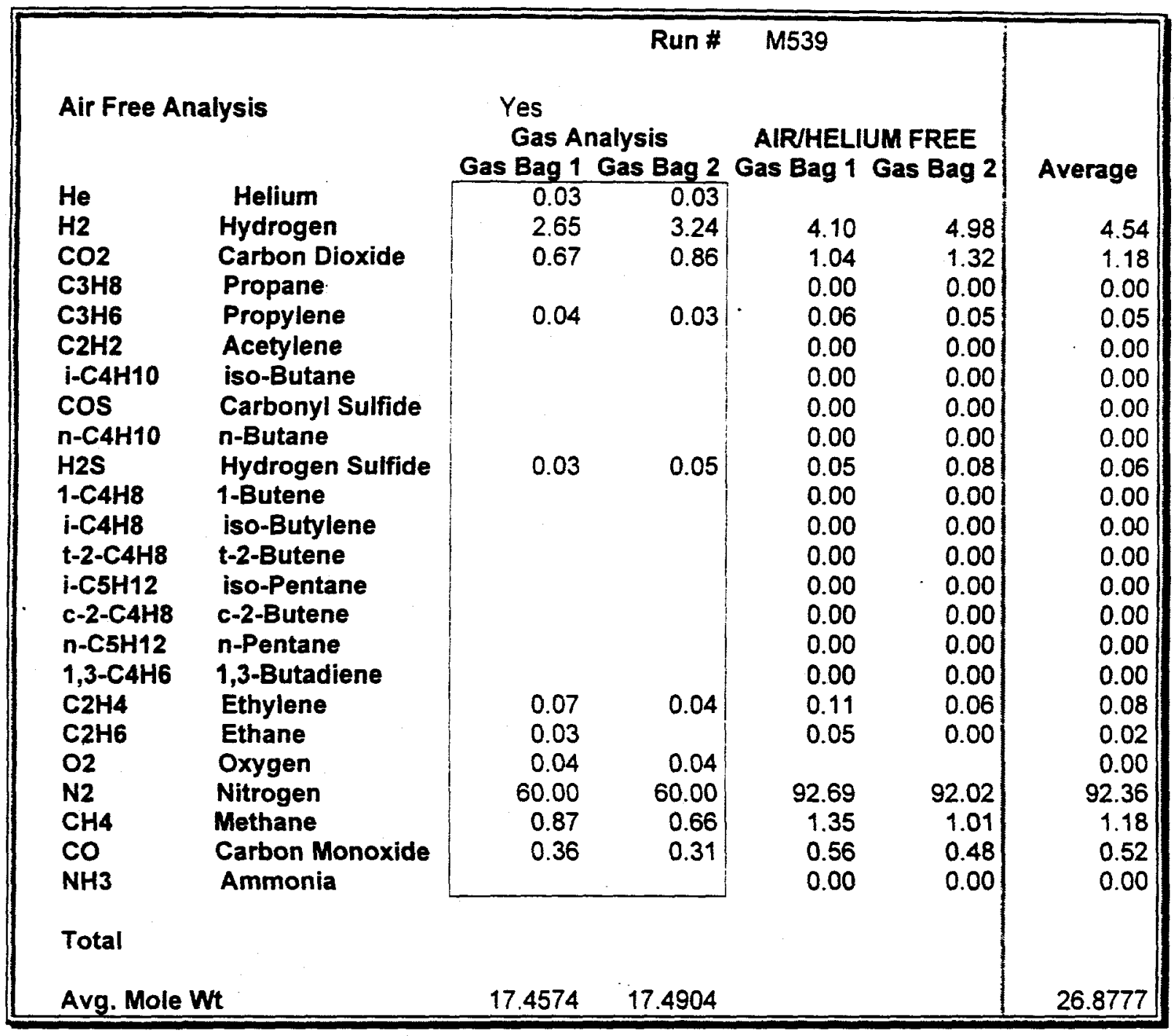

